Katarzyna Haverkamp

Bestimmungsfaktoren der Vertragsforschung in Deutschland 
CEGE-SCHRIFTEN

Katarzyna Haverkamp

\section{Bestimmungsfaktoren der Vertragsforschung in Deutschland}

Die offiziellen Daten zur FuE-Tätigkeit der deutschen Wirtschaft zeigen eine zunehmende Bedeutung der Vertragsforschung als einer Organisationsalternative für die Abwicklung von FuE-Aktivitäten. Der Anteil der für den externen Bezug von FuE-Dienstleistungen aufgewendeten Mittel im Verhältnis zu den gesamten FuE-Aufwendungen der Unternehmen ist zwischen 1979 und 2003 von etwa $5 \%$ auf über $18 \%$ angestiegen. Die Gründe für die verstärkte Fremdvergabe von FuE-Aufträgen an unternehmensexterne Partner sind jedoch bisher nur unzureichend untersucht worden. In dieser Studie werden die Determinanten der Vertragsforschungsaktivitäten theoretisch untersucht und auf der Grundlage des Mannheimer Innovationspanels auf Unternehmensebene geschätzt.

Katarzyna Haverkamp wurde 1978 in Bialystok (Polen) geboren. Nach dem Studium in Warschau (1997-2002) folgte ein Promotionsstudium (2003-2006) an der Universität Göttingen, welches sie Ende 2006 abschloss. 
Bestimmungsfaktoren der Vertragsforschung in Deutschland 


\title{
Cege-Schriften
}

Center for Globalization and Europeanization of the Economy Centrum für Globalisierung und Europäisierung der Wirtschaft

Georg-August-Universität Göttingen

\section{Band 12}

\author{
Herausgegeben von \\ Kilian Bizer, Stephan von Cramon-Taubadel, \\ Günter Gabisch, Helmut Hesse, Andreas Oestreicher, \\ Renate Ohr, Gerhard Rübel, \\ Hermann Sautter, Robert Schwager, \\ Stefan Tangermann
}

Verantwortlicher Herousgeber für diesen Band:

Kilian Bizer

\section{(3) \\ PETER LANG}

Frankfurt am Main - Berlin - Bern - Bruxelles - New York - Oxford - Wien 


\title{
Katarzyna Haverkamp
}

\section{Bestimmungsfaktoren der Vertragsforschung in Deutschland}

\author{
Eine theoretische und \\ ökonometrische Analyse
}

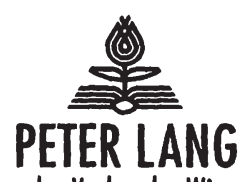


Bibliografische Information der Deutschen Nationalbibliothek Die Deutsche Nationalbibliothek verzeichnet diese Publikation in der Deutschen Nationalbibliografie; detaillierte bibliografische Daten sind im Internet über <http://www.d-nb.de> abrufbar.

Open Access: The online version of this publication is published on www.peterlang.com and www.econstor.eu under the international Creative Commons License CC-BY 4.0. Learn more on how you can use and share this work: http://creativecommons.org/licenses/ by/4.0.

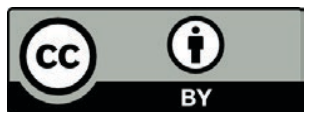

This book is available Open Access thanks to the kind support of ZBW - Leibniz-Informationszentrum Wirtschaft.

Gedruckt auf alterungsbeständigem, säurefreiem Papier.

Zugl.: Göttingen, Univ., Diss., 2006

\author{
D 7
}

ISSN 1617-741X

ISBN 978-3-631-56714-2

ISBN 978-3-631-75689-8 (eBook)

(c) Peter Lang $\mathrm{GmbH}$

Internationaler Verlag der Wissenschaften

Frankfurt am Main 2007

Alle Rechte vorbehalten.

Das Werk einschließlich aller seiner Teile ist urheberrechtlich geschützt. Jede Verwertung außerhalb der engen Grenzen des

Urheberrechtsgesetzes ist ohne Zustimmung des Verlages unzulässig und strafbar. Das gilt insbesondere für

Vervielfältigungen, Übersetzungen, Mikroverfilmungen und die Einspeicherung und Verarbeitung in elektronischen Systemen.

Printed in Germany 123457

www.peterlang.de 


\section{Vorwort der Herausgeber}

Die Organisationsstruktur industrieller Forschung und Entwicklung wird zunehmend arbeitsteiliger und stellt eine besondere Herausforderung für die wirtschaftswissenschaftliche Forschung dar. Im Mittelpunkt der vorliegenden Arbeit steht die sogenannte Vertragsforschung, welche die Autorin als eine gegen Bezahlung erbrachte Forschungs- und Entwicklungsdienstleistung und somit Markttransaktion versteht. Die Vergütung für eine klar umrissene und vereinbarte Auftragsforschung ist somit das konstituierende Merkmal. Dieser Untersuchungsgegenstand ist bis zu diesem Zeitpunkt noch keiner systematischen Analyse unterzogen worden. Er erlangt seine Bedeutung im Zeichen von Alternativen der Forschungs- und Entwicklungstätigkeit, die den Unternehmen stets zur Verfügung stehen.

Als Ergebnis einer rigorosen Auswertung an der Vorderfront des Wissenstandes gelangt die Autorin zu zehn theoriegeleiteten Hypothesen, die differenziert dem Transaktionskostenansatz, dem Property-Rights-Ansatz und strategieorientierten Modellen der Industrieökonomik zugeordnet werden, bevor sie einer ökonometrischen Analyse unterzogen werden. Hierbei kommt der Autorin das Mannheimer Innovationspanel als systematische Erhebung des Innovationsverhaltens der deutschen Wirtschaft zugute, die vom Zentrum für europäische Wirtschaftsforschung (ZEW) in Zusammenarbeit mit dem infas-Institut für angewandte Sozialwissenschaft im Auftrag des Bundesministeriums für Bildung und Forschung seit 1993 durchgeführt wird.

Gegen diesen Hintergrund deckt die Autorin die relevanten Kausalitätsbereiche $a b$ und kann überzeugende Ergebnisse vorlegen. Ihre Synthese von Theorie und Praxis unter Verwendung eines modernen Instrumentariums trägt dem Umstand der Unvollständigkeit von Verträgen und den Implikationen von Externalitäten derart Rechnung, dass die Gründe dafür offengelegt werden, dass Unternehmen immer stärker auf unternehmensextern vorhandene 
Kompetenzen zurückgreifen. Frau Haverkamp hat Neuland erschlossen und die allgemeine Innovationsforschung nachhaltig beflügelt.

Prof. Dr. Wolfgang König

Prof. Dr. Kilian Bizer 


\section{Danksagung}

Die vorliegende Arbeit wurde im Wintersemester 2005/06 von der wirtschaftswissenschaftlichen Fakultät der Georg-August-Universität Göttingen als Dissertation angenommen. Meinem Doktorvater, Herrn Prof. Dr. Wolfgang König, gilt mein besonderer Dank für die Aufnahme als Promotionskandidatin, die vielseitige Förderung während der Promotionszeit und den großzügig gewährten Freiraum für die eigenständige Forschung. Herrn Prof. Dr. Kilian Bizer möchte ich für das Interesse an meiner Arbeit und die freundliche Übernahme des Zweitgutachtens danken. Mein Dank gebührt auch Herrn Prof. Dr. Dr. h.c. Jürgen Bloech, der sich als Drittprüfer im Rigorosum zur Verfügung stellte.

Dem Zentrum für Europäische Wirtschaftsforschung in Mannheim danke ich für die Bereitstellung der Daten des Mannheimer Innovationspanels, ohne die der empirische Teil meiner Arbeit nicht möglich gewesen wäre. Bei Frau Dr. Sandra Gottschalk möchte ich mich ausdrücklich für die stets sehr freundliche Betreuung, insbesondere auch während der Gastaufenthalte am ZEW Mannheim, bedanken. Schließlich sei auch allen Mitarbeitern des Volkswirtschaftlichen Institutes für Mittelstand und Handwerk an der Universität Göttingen (ifh Göttingen) gedankt für die freundliche Begleitung und Unterstützung während meiner Promotionszeit, Anja Sölter in besonderer Weise für die stets sehr geduldige Auseinandersetzung mit dem Manuskript.

Die Fertigstellung der Arbeit wurde durch die finanzielle Förderung seitens der Konrad-Adenauer-Stiftung ermöglicht. Auch dieser gilt mein ausgesprochener Dank. 
Katarzyna Haverkamp - 978-3-631-75689-8

Downloaded from PubFactory at 01/11/2019 02:56:45AM

via free access 


\section{Inhaltsverzeichnis}

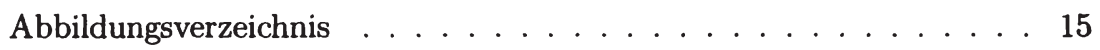

Tabellenverzeichnis . . . . . . . . . . . . . . . 17

Abkürzungsverzeichnis . . . . . . . . . . . . . . 19

1 Einleitung 21

1.1 Problemstellung und Zielsetzung . . . . . . . . . . . . 21

1.2 Generelle Trends im ökonomischen und technologischen Umfeld 24

1.3 Stand der Forschung . . . . . . . . . . . . . . 27

1.4 Aufbau der Arbeit und Gang der Untersuchung . . . . . . . 30

2 Grundlegende Begriffe, Abgrenzungen und Klassifikationen 33

2.1 Zum Begriff Forschung und Entwicklung . . . . . . . . . . 34

2.1.1 Merkmale von Forschungs- und Entwicklungsaktivitäten 34

2.1.2 Klassifikationsmöglichkeiten . . . . . . . . . 37

2.1.3 Forschung und Entwicklung im Innovationsprozess . . . . 38

2.2 Einordnung und Abgrenzung der Vertragsforschung . . . . . . 40

2.3 Eigenschaften des Gutes Wissen . . . . . . . . . . . . 44

2.3.1 Wissen als Information . . . . . . . . . . 45

2.3.2 Wissen als Know-how . . . . . . . . . . . . 46 
3 Bestimmungsgründe der FuE-Tätigkeit im Wirtschaftssektor 49

3.1 Messung der FuE-Tätigkeit . . . . . . . . . . . . . 51

3.2 Schumpetersche Erkenntnisse und Neo-Schumpeter-Hypothesen 56

3.2.1 Unternehmensgröße und Forschungstätigkeit . . . . . . 59

3.2.2 Marktstruktur und Forschungstätigkeit . . . . . . . 65

3.3 Unternehmensinterne Determinanten der FuE-Tätigkeit . . . . . 69

3.3 .1 Finanzierung $\ldots \ldots \ldots \ldots \ldots$

3.3 .2 Diversifizierung . . . . . . . . . . . . 73

3.3.3 Exporttätigkeit . . . . . . . . . . . . 75

3.3.4 Konzernzugehörigkeit . . . . . . . . . . . . 76

3.4 Industrieökonomische Determinanten der FuE-Tätigkeit . . . . . 77

3.4 .1 Nachfrage . . . . . . . . . . . . . 77

3.4 .2 Aneignungsbedingungen . . . . . . . . 80

3.4.2.1 Studien zu alternativen Schutzmechanismen . . 82

3.4.2.2 Empirische Evidenz . . . . . . . . . . 85

3.4.3 Technologische Möglichkeiten . . . . . . . . . 88

3.4.3.1 Die Typologie von Pavitt . . . . . . . . 89

3.4.3.2 Empirische Evidenz _. . . . . . . . . . . 91

3.5 Zusammenfassung der Ergebnisse . . . . . . . . . . . . . 94

4 Vertragsforschung aus theoretischer Perspektive 101

4.1 Auswahl theoretischer Ansätze . . . . . . . . . . . . . . 102

4.2 Der Transaktionskostenansatz . . . . . . . . . . . . . . . 103

4.2.1 Grunderkenntnisse von Coase . . . . . . . . . . . . . 104

4.2.2 Williamsons vertragstheoretische Weiterentwicklung . . . 106

4.2.2.1 Zum Begriff der Transaktionskosten . . . . . . . 106

4.2.2.2 Einflussgrößen auf die Höhe der Transaktionskosten . . . . . . . . . . . . . . 108 
4.2.2.3 Komparative Analyse der Koordinationsformen 112

4.2.3 Transaktionskostenorientierte Analyse der Vertragsforschung . . . . . . . . . . . . . . . 114

4.2.3.1 Zur Bedeutung der Vertragsunvollständigkeit . 115

4.2.3.2 Zum Einfluss der Transaktionseigenschaften . . 117

4.2.3.3 Zur Frage der Koordinationsfähigkeit . . . . . . 119

4.2.4 Grenzen der transaktionskostenorientierten Analyse . . . 120

4.3 Der Property-Rights-Ansatz . . . . . . . . . . . . . . . . 123

4.3.1 Definitorische Grundlagen . . . . . . . . . . . 124

4.3.2 Die zu modellierende Situation . . . . . . . . . . 125

4.3.3 Modellanalyse . . . . . . . . . . . . . . . . 128

4.3.3.1 Modellannahmen . . . . . . . . . . . . . 128

4.3.3.2 Aufteilung der Ex-post-Transaktionsrente . . . 129

4.3.3.3 Investitionsentscheidungen . . . . . . . . 130

4.3.3.4 Analyse der optimalen Eigentumsstruktur . . . 132

4.3.4 Implikationen im Kontext der Vertragsforschung . . . . . 133

4.3.4.1 Zur Bedeutung der Vertragsunvollständigkeit . 133

4.3.4.2 Ressourcen und Organisationsentscheidungen . 135

4.4 Strategieorientierte Modelle der Industrieökonomik . . . . . . 137

4.4.1 Spillovers und FuE-Kooperation . . . . . . . . . . 138

4.4.1.1 Das Grundmodell von d'Aspremont und Jacquemin . . . . . . . . . . . . 139

4.4.1.2 Modellierung von Spillovers . . . . . . . . . 142

4.4.2 Zur Frage der Absorptionsfähigkeit . . . . . . . . . 148

4.4.2.1 Die Grunderkenntnisse von Cohen und Levinthal148

4.4.2.2 Die Modellierung der Absorptionsfähigkeit . . . 150

4.4.3 Implikationen im Kontext der Vertragsforschung . . . . . 152

4.5 Zusammenfassung und abschließende Bemerkungen . . . . . 155

4.5.1 Ähnlichkeiten und Unterschiede . . . . . . . . . . . 155

4.5.2 Zusammenführung der Hypothesen . . . . . . . 157 
5.1 Anmerkungen zu Datenquellen . . . . . . . . . . . . . . . 162

5.1.1 Die deutsche FuE-Statistik . . . . . . . . . . . . 162

5.1.2 Das Mannheimer Innovationspanel (MIP) . . . . . . 165

5.2 Ergebnisse bisheriger empirischer Studien . . . . . . . . . 167

5.2.1 Evaluierung der Fördermaßnahme „Externe Vertragsforschung" . . . . . . . . . . . . . . . 168

5.2.2 Sonderauswertung der Wissenschaftsstatistik . . . . . 171

5.2.3 Ifo-Befragung im Verarbeitenden Gewerbe . . . . . . . 174

5.2.4 Vertragsforschungsanbieter aus den neuen Bundesländern 178

5.2 .5 Zwischenfazit . . . . . . . . . . . . 179

5.3 Modellschätzungen I (MIP 1993) . . . . . . . . . . . 181

5.3.1 Anmerkungen zur Stichprobe . . . . . . . . . 181

5.3.2 Koordinationsformen für FuE-Leistungen . . . . . . . . 183

5.3.3 Determinanten der Durchführung von FuE-Aufträgen . . 186

5.3.3.1 Spezifikation von Determinanten . . . . . . 187

5.3.3.2 Schätzergebnisse . . . . . . . . . . . 193

5.3.4 Determinanten der Vergabe von FuE-Aufträgen . . . . 197

5.3.4.1 Spezifikation von Determinanten der Auftragsvergabe . . . . . . . . . . . . . 197

5.3.4.2 Schätzergebnisse . . . . . . . . . 200

5.4 Modellschätzungen II (MIP 2003) . . . . . . . . . . 205

5.4.1 Anmerkungen zur Stichprobe . . . . . . . . . 205

5.4 .2 Deskriptive Statistik . . . . . . . . . . . 208

5.4.2.1 Auftragsvergabe und Unternehmensgröße _ . . 208

5.4.2.2 Auftragsvergabe und Eigentumsverhältnisse der Unternehmen . . . . . . . . . . . . . . . 209 
5.4.2.3 Auftragsvergabe und Forschungsintensität der Unternehmen . . . . . . . . . . . . . 210

5.4.2.4 Auftragsvergabe nach alten und neuen Bundesländern . . . . . . . . . . 211

5.4.2.5 Auftragsvergabe nach Wirtschaftszweigen der Unternehmen . . . . . . . . . . . . 211

5.4.3 Determinanten der Auftragsvergabe . . . . . . . . . . 212

5.4.3.1 Spezifikation der Determinanten . . . . . . . 214

5.4.3.2 Schätzergebnisse . . . . . . . . . . 216

5.5 Diskussion der Ergebnisse $\ldots \ldots \ldots \ldots \ldots \ldots$

6 Zusammenfassung und Ausblick 231

6.1 Zusammenfassung der Ergebnisse . . . . . . . . . . . . 231

6.2 Wirtschaftspolitische Implikationen . . . . . . . . . 238

6.3 Implikationen für die Innovationsforschung . . . . . . . . . . . 240 
Katarzyna Haverkamp - 978-3-631-75689-8

Downloaded from PubFactory at 01/11/2019 02:56:45AM

via free access 


\section{Abbildungsverzeichnis}

1.1 FuE-Gesamtaufwendungen und externe FuE-Aufwendungen der Unternehmen 1979-2003 . . . . . . . . . . . . . . . 22

1.2 Struktur der externen FuE-Aufwendungen der Unternehmen nach Auftragnehmern 1979-2003 . . . . . . . . . . 23

2.1 Der Systematisierungsansatz von Schneider und Zieringer . . . . 41

4.1 Ablauf der Transaktionsbeziehung im Hartschen Modell . . . . . 128 
Katarzyna Haverkamp - 978-3-631-75689-8

Downloaded from PubFactory at 01/11/2019 02:56:45AM

via free access 


\section{Tabellenverzeichnis}

3.1 Indikatoren der Innovationstätigkeit . . . . . . . . . . . . 54

4.1 Eigenschaften von Markt, Unternehmen und hybriden Koordinationsformen . . . . . . . . . . . . . . . 114

4.2 Eigenstumsstrukturen (ownership structures) im Hartschen Modell . . . . . . . . . . . . . . . . 126

5.1 Gründe für die Vergabe von Entwicklungsaufträgen . . . . . . 169

5.2 Relativer Anteil und Struktur externer FuE-Aufwendungen der Unternehmen nach Wirtschaftszweigen (Anteile in \%) . . . . 172

5.3 Relativer Anteil und Struktur externer FuE-Aufwendungen nach Forschungsintensitäten und Konzernabhängigkeit der Unternehmen (Anteile in \%) . . . . . . . . . . . 174

5.4 Motive der Unternehmen bei der Nutzung externer FuEDienstleistungen nach Auftragnehmertypen (Häufigkeit der Nennung in \%) . . . . . . . . . . . . . . 175

5.5 Motive der Unternehmen bei der Durchführung von FuEDienstleistungen für externe Auftraggeber (Häufigkeit der Nennung in \%) . . . . . . . . . . . . . . 177

5.6 Zusammensetzung der Stichproben (MIP 1993) . . . . . . . . 182

5.7 Verbreitung der Formen des Erwerbs neuen technischen Wissens 184

5.8 Verbreitung der Formen der Weitergabe neuen technischen Wissens . . . . . . . . . . . . . . . . 185

5.9 Spezifikation der Determinanten der Durchführung von FuELeistungen . . . . . . . . . . . . . . . . . . . 188 
5.10 Ergebnisse der Faktoranalyse der Schutzmaßnahmen . . . . . . 190

5.11 Ergebnisse der Faktoranalyse der unternehmensexternen Wissensquellen . . . . . . . . . . . . . . . . . . 191

5.12 Schätzergebnisse der Determinanten der Durchführung von FuE-Aufträgen . . . . . . . . . . . . . . . . . 194

5.13 Spezifikation der Determinanten der Auftragsvergabe (MIP 1993) 198

5.14 Schätzergebnisse der Determinanten der Auftragsvergabe (MIP 1993) . . . . . . . . . . . . . . . . 202

5.15 Zusammensetzung der Stichproben (MIP 2003) . . . . . . . 207

5.16 Beteiligung an Vertragsforschung nach Betriebsgrößenklassen . . 209

5.17 Beteiligung an Vertragsforschung nach Eigentumsverhältnissen . 209

5.18 Beteiligung an Vertragsforschung nach Forschungsintensität der Unternehmen . . . . . . . . . . . . . . . . . . . . 210

5.19 Beteiligung an Vertragsforschung nach Regionen (alte/neue Bundesländer $) \ldots \ldots \ldots \ldots . \ldots \ldots \ldots$

5.20 Beteiligung an Vertragsforschung nach Wirtschaftszweigen der Unternehmen . . . . . . . . . . . . . . . . . . . . 213

5.21 Spezifikation der Regressoren (MIP 2003) . . . . . . . . . 215

5.22 Schätzergebnisse der Determinanten der Auftragsvergabe (MIP 2003) . . . . . . . . . . . . . . . 217

5.23 Schätzergebnisse der Determinanten der Auftragsvergabe (MIP 2003, ohne wissensintensive Branchen des Dienstleistungssektors) 220 


\section{Abkürzungsverzeichnis}

\begin{tabular}{ll} 
Abb. & Abbildung \\
AJ-Modell & das Modell von d'Aspremont und Jacquemin (1988) \\
BIC & Bayessches Informationskriterium \\
BMBF & Bundesministerium für Bildung und Forschung \\
BMWA & Bundesministerium für Wirtschaft und Arbeit \\
BMWi & Bundesministerium für Wirtschaft und Technologie \\
bzw. & beziehungsweise \\
CIS & Community Innovation Survey \\
d.h. & das heißt \\
DIW & Deutsches Institut für Wirtschaftsforschung \\
DM & Deutsche Mark \\
EDV & Herstellung von Büromaschinen, Datenverarbeitungsgeräten \\
& und -einrichtungen \\
et al. & et alii \\
EG & Europäische Gemeinschaften \\
EU & Europäische Union \\
EUR & Euro \\
Eurostat & das Statistische Amt der Europäischen Gemeinschaften \\
f./ff. & folgende/fortfolgende \\
FhG & Fraunhofer-Gesellschaft \\
FuE & Forschung und Entwicklung \\
Hrsg. & Herausgeber \\
IAB & Institut für Arbeitsmarkt- und Berufsforschung \\
i.d.R. & in der Regel \\
ISI & Institut für System- und Innovationsforschung \\
& der Fraunhofer-Gesellschaft \\
KfW & Kreditanstalt für Wiederaufbau \\
Kfz & Kraftfahrzeug \\
\hline
\end{tabular}


KMU

KMZ-Modell

Koeff.

KOF/ETH

M\&A

Mrd.

MIP

Mio.

MMSR

MSMSS

MW

n.a.

NBER

$\mathrm{NSH}$

OECD

o.g.

PRA

$\mathrm{R} \& \mathrm{D}$

RJV

$\mathrm{S}$.

SD

sog.

Tab.

TK

TKA

Tsd.

u.a.

US

UNCTAD

usw.

vgl.

WZ

WZ 1993

WZ 2003

z.B.

ZEW
Klein- und Mittelunternehmen

das Modell von Kamien, Muller und Zang (1992)

Koeffizient

Konjunkturforschungsstelle

der Eidgenössischen Technischen Hochschule (ETH) Zürich mergers and acquisitions

Milliarde

Mannheimer Innovationspanel

Millionen

Medizin-, Meß-, Steuer-, und Regelungstechnik

Möbel, Schmuck, Musikinstrumente, Sportgeräte, Spielwaren

Mittelwert

nicht anwendbar

National Bureau of Economic Research

Neo-Schumpeter-Hypothese

Organisation for Economic Cooperation and Development oben genannt

Property-Rights-Ansatz

research and development

research joint venture

Seite

Standardabweichung

sogenannte

Tabelle

Transaktionskosten

Transaktionskostenansatz

Tausend

und andere

United States

United Nations Conference on Trade and Development

und so weiter

vergleiche

Wirtschaftszweig

Klassifikation der Wirtschaftszweige, Ausgabe 1993

Klassifikation der Wirtschaftszweige, Ausgabe 2003 zum Beispiel

Zentrum für Europäische Wirtschaftsforschung $\mathrm{GmbH}$ 


\section{Kapitel 1}

\section{Einleitung}

\subsection{Problemstellung und Zielsetzung}

Seit mehreren Jahren zeigt sich eine Veränderung der Organisationsstruktur industrieller Forschung und Entwicklung (FuE). Galt lange Zeit das Paradigma der isolierten FuE-Aktivitäten der im Wettbewerb stehenden Unternehmen, die das innerbetrieblich erarbeitete Wissen intern in Produktionsprozessen verwerten und vor der unfreiwilligen Diffusion durch Patentierung schützen, so lässt sich neuerdings eine Tendenz zu zunehmend arbeitsteiligen FuE-Prozessen und einer verstärkten Verflechtung zwischen den Wirtschaftsakteuren beobachten. Zwei Erscheinungsmuster dieser Entwicklung können hervorgehoben werden.

Zum einen lässt sich im Zeitablauf ein bedeutsamer Anstieg der Anzahl von Kooperationsbeziehungen feststellen. Die Dynamik dieser Entwicklung kann mit den Daten einer Studie von Hagedoorn (2002) exemplifiziert werden, einer der wenigen Langzeitbetrachtungen der Kooperationstätigkeit im FuEBereich. Diese Studie dokumentiert die Entwicklung der Anzahl der weltweit jährlich neu eingegangenen vertraglich fixierten FuE-Kooperationen; das Phänomen der informalen Zusammenarbeit zwischen FuE-betreibenden Unternehmen wird folglich durch diese Statistik nicht erfasst. Trotzdem zeigt sich: Während in den 60er Jahren des vergangenen Jahrhunderts die Anzahl der jährlich neu eingegangenen, in der MERIT-CATI-Datenbank ${ }^{1}$ registrierten FuE-Kooperationen um den Wert von 10 variierte, und in der Mitte der 70er Jahre die Marke von 50 erreichte, überstieg sie zu Ende der 80er Jahre den

${ }^{1}$ Zur genauen Beschreibung der Datenbasis vgl. Hagedoorn (2002), Anhang A, S. 491. 
Wert von ca. 500 Vereinbarungen, den sie auch - nach einigen Schwankungen in den 90er Jahren - 1997 aufwies.

Zum anderen kann eine wachsende Bedeutung der Auftragsforschung festgestellt werden. Im Gegensatz zur FuE-Kooperation, die als Zusammenarbeit von unabhängigen Unternehmen zum Zweck der gemeinsamen Wissensgenerierung definiert werden kann, handelt es sich bei der Auftragsforschung um eine gegen Bezahlung erbrachte FuE-Dienstleistung und damit um eine Markttransaktion. Die wachsende Bedeutung der zwischenbetrieblichen Tauschbeziehungen auf dem FuE-Markt lässt sich in Deutschland anhand von Daten der offiziellen FuE-Statistik verfolgen. ${ }^{2}$ In der FuE-Statistik werden die vom Wirtschaftssektor getätigten Zahlungen für FuE-Aufträge als „Aufwendungen für externe Forschung und Entwicklung“ erfasst; entsprechende Daten liegen seit 1979 vor.

Abbildung 1.1: FuE-Gesamtaufwendungen und externe FuE-Aufwendungen der Unternehmen 1979-2003

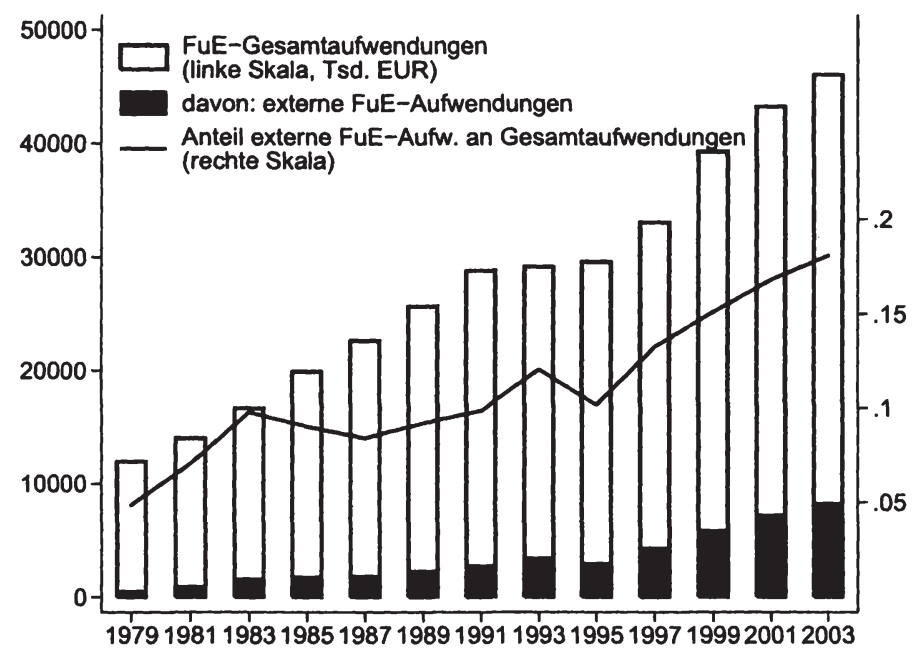

Quelle: Wissenschaftsstatistik; eigene Darstellung.

In Abb. 1.1 ist die Entwicklung der externen FuE-Aufwendungen der deutschen Unternehmen im Zeitraum von 1979 bis 2003 dargestellt. Die Abbildung

${ }^{2}$ Für die Daten zur Entwicklung des FuE-Marktes in der britischen Volkswirtschaft vgl. Howells (1997), S. 5 ff., für Daten zur US-amerikanischen Volkswirtschaft vgl. Jankowski (2001), S. $329 \mathrm{ff}$. 
lässt eine deutliche Vergrößerung des Marktes für FuE-Dienstleistungen erkennen: Wendeten die Unternehmen im ersten Beobachtungsjahr 1979 ca. 0,6 Mrd. EUR für externe FuE auf, so ist dieser Betrag bis 2003 auf über 8,3 Mrd EUR gestiegen (linke Skala). Der Anteil der Aufwendungen für die von Dritten erbrachten Dienstleistungen an den FuE-Gesamtaufwendungen der Unternehmen (rechte Skala) stieg von ca. 5\% (1979) auf über 18\% (2003). In der Entwicklung des Anteils der Aufwendungen für FuE-Dienstleistungen an den Gesamtaufwendungen ist eine deutliche Beschleunigung ab der zweiten Hälfte der 90er Jahre erkennbar; externe Forschung und Entwicklung trug in dem Zeitraum zwischen 1997 und 2003 zu fast 30\% zum gesamten Bruttoanstieg der FuE-Aufwendungen des Wirtschaftssektors bei. ${ }^{3}$

Abbildung 1.2: Struktur der externen FuE-Aufwendungen der Unternehmen nach Auftragnehmern 1979-2003

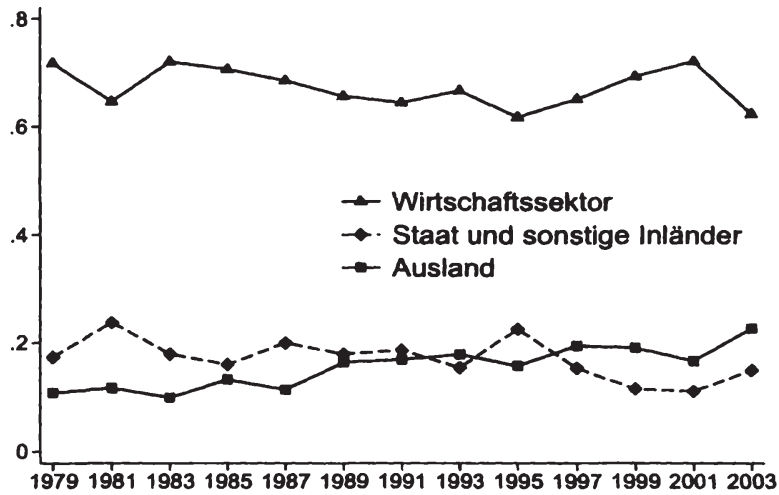

Quelle: Wissenschaftsstatistik; eigene Darstellung.

Ferner zeigt die deutsche FuE-Statistik: Empfänger der Zahlungen für die externe FuE-Aufwendungen sind nicht - wie es oft in der öffentlichen Wahrnehmung angenommen wird - staatlich finanzierte wissenschaftliche Einrichtungen (Hochschulen bzw. Forschungsinstitute), sondern vorwiegend Akteure des Wirtschaftssektors (Abb. 1.2). Mit über 60\%-igem Anteil dominiert der Wirtschaftssektor die Angebotsseite des Marktes für FuE-Dienstleistungen. Die Bedeutung der sonstigen Inländer - darunter fallen sowohl Hochschulinstitute und staatlich finanzierte Forschungseinrichtungen als auch private Organisatio-

3 Vgl. Legler/Grenzmann/Marquardt (2006), S. 53. 
nen ohne Erwerbszweck - lässt im Zeitablauf insbesondere gegenüber den Anbietern aus dem Ausland nach. Bei Letzteren handelt es sich jedoch Schätzungen zufolge ebenfalls mehrheitlich um Akteure des Wirtschaftssektors. ${ }^{4}$

Die angeführten Statistiken zeigen deutlich, dass die auf individuellen Wettbewerb ausgerichteten Überlegungen nicht mehr geeignet sind, das durch wachsende Verflechtungen geprägte Bild der industriellen Forschungs- und Entwicklungstätigkeit zu erklären. Die Analyse der zunehmend arbeitsteiligen FuEProzesse stellt eine Herausforderung für die wirtschaftswissenschaftliche Forschung dar. Während jedoch Kooperationsentscheidungen und -strategien der Unternehmen in der neueren innovationsökonomischen Literatur sehr intensiv der theoretischen und empirischen Analyse zugeführt werden ${ }^{5}$, existieren bis heute nur einige wenige Beiträge, die sich explizit mit dem Phänomen der dynamischen Entwicklung des Marktes für die FuE-Dienstleistungen auseinandersetzen.

Angesichts dieses Defizits wird in der vorliegenden Arbeit der Frage nachgegangen, inwieweit die beobachtbare Tendenz zur verstärkten Inanspruchnahme des Instruments der Vertragsforschung einer wirtschaftswissenschaftlichen Analyse zugeführt werden kann. Die Erklärung für die Herausbildung des Marktes für FuE-Dienstleistungen wird somit nicht primär in den weltweiten Veränderungen der Rahmenbedingungen für wirtschaftliche Aktivität gesucht. Vor dem Hintergrund der zunehmenden Dynamik und Komplexität des Unternehmungsumfelds sollen die einzelwirtschaftlichen Anpassungsprozesse, vor allem die Entscheidungsmuster der gewinngesteuerten Wirtschaftsakteure untersucht werden. Es ist das Ziel der vorliegenden Arbeit, in dieser Hinsicht die Bestimmungsfaktoren der Vertragsforschung auf der Unternehmensebene zu identifizieren und einer empirischen Prüfung zu unterziehen.

\subsection{Generelle Trends im ökonomischen und technologischen Umfeld}

Hintergrund für die Herausbildung und dynamische Entwicklung des Marktes für FuE-Dienstleistungen bilden die in den vorangegangenen Jahren stattgefundenen weltweiten Veränderungen im ökonomischen und technologischen

${ }^{4}$ Vgl. Legler/Grenzmann/Marquardt (2006), S. 55.

5 Für eine synthetische Aufarbeitung der vorliegenden theoretischen und empirischen Literatur vgl. Hagedoorn/Link/Vonortas (2000) und Veugelers (1998). 
Umfeld der einzelwirtschaftlichen Aktivität. Der durch Globalisierungsprozesse geprägte Wandel von Wettbewerbsbedingungen ist hierbei an erster Stelle zu nennen. Stetig steigender Wettbewerbsdruck löste Anpassungsprozesse in Unternehmen aus, die vor allem in der Tendenz zur Reorganisation betrieblicher Leistungserstellungsprozesse zum Ausdruck kamen. Die einzelwirtschaftliche Suche nach der optimalen Balance zwischen den Spezialisierungsvorteilen der arbeitsteiligen Produktion und den Rationalisierungsvorteilen integrierter Leistungserstellung $^{6}$ führte zu einer Welle internationaler Zusammenschlüsse der bisher selbstständigen Unternehmen einerseits ${ }^{7}$ und zum allgemeinen Bedeutungszuwachs von Outsourcingstrategien ${ }^{8}$ andererseits.

Die Vertiefung der Arbeitsteilung infolge der Öffnung der Weltmärkte hat in der Folge ein komplexes Beziehungsgeflecht zwischen den Wirtschaftsakteuren entstehen lassen: Die spezialisierten, „schlanken “9 Unternehmen sind jetzt auf enge Zusammenarbeit bzw. Markttransaktionen mit ausgegliederten Unternehmenseinheiten, Zulieferern und Kunden angewiesen; den entstandenen Abhängigkeiten versuchen sie durch die Bildung strategischer Allianzen und vertraglich geregelter Kooperationen zu entgegnen. ${ }^{10}$ Die durch Konzentrationsprozesse gewachsenen Wirtschaftseinheiten greifen verstärkt auf marktähnliche Koordinationsmechanismen (sog. interne Märkte ${ }^{11}$ ) zurück; die Fortschritte in der Informations- und Kommunikationstechnologie erlauben dabei die Bewältigung der gestiegenen Koordinationsbedarfe.

Unter den im ökonomischen Umfeld auftretenden Veränderungen ist nicht zuletzt der Wandel von einem Verkäufer- hin zu einem Käufermarkt zu nennen $^{12}$, der die innovierenden Unternehmen in einen „permanenten Innovationswettbewerb“13 versetzt und den Druck auf die zügige Umsetzung von FuEErgebnissen in ein wirtschaftlich verwertbares Produkt erhöht. ${ }^{14}$

6 Vgl. Kinkel/Lay (2003), S. 2.

7 Vgl. insbesondere UNCTAD (2000), Kap. IV.

8 Vgl. Grossman/Helpman (1999), Grossman/Helpman (2005), Feenstra (1998).

9 Vgl. Kinkel/Lay (2003), S. 2.

${ }^{10}$ Zum in diesem Zusammenhang entstandenen Begriff des „alliance capitalism“ vgl. Dunning (1997). Zu strategischen Allianzen im FuE-Bereich vgl. u.a. Hagedoorn (1995), Rothaermel (2001).

11 Zum Phänomen der internen Märkte in Forschung und Entwicklung vgl. insbesondere Völker/Kasper (2004).

12 Vgl. Gauglitz-Lüter (1998), S. 100.

${ }^{13}$ Koschatzky/Reinhard/Grenzmann (2003), S. 149.

14 Vgl. UNCTAD (2005), S. 171, Eichin (1995), S. 66, Koschatzky/Reinhard/Grenzmann 
Neben den Veränderungen im ökonomischen Umfeld wird auch der Wandel der technologischen Rahmenbedingungen als eine treibende Kraft der Tendenz zur Veränderung der Organisationsstruktur der FuE-Tätigkeit im Wirtschaftssektor angesehen. An erster Stelle sei hierbei die Verkürzung der Technologieund Produktlebenszyklen genannt. ${ }^{15}$ Kürzer werdende Lebenszyklen von Produkten und Technologien bedeuten aus privatwirtschaftlicher Sicht, dass die Zeitspanne, in der die Unternehmen die entstandenen FuE-Kosten amortisieren und Monopolgewinne realisieren können, kleiner geworden ist. Erschwert wird die Rückfinanzierung der Innovationsprojekte zudem neuerdings durch die insgesamt gestiegenen Kosten der FuE-Tätigkeit. ${ }^{16}$

Ferner verweist die einschlägige Literatur auf die zunehmende Bedeutung der integrativen Technologien. ${ }^{17} \mathrm{Da}$ die innovativen Produkte zunehmend auf mehreren Technologien beruhen, die selbst in großen Unternehmen nicht vollständig vertreten sind, ergibt sich für einzelne Wirtschaftsakteure die Notwendigkeit, auf die spezifischen FuE-Kompetenzen unternehmensexterner Partner zurückzugreifen. ${ }^{18}$ Erst die Integration externen Wissens ermöglicht die Erarbeitung neuer komplexer Verfahren und Produkte. Ferner ist die Wissenschaftsbindung der zukunftsorientierten Technikgebiete gestiegen, was die Unternehmen mit Institutionen zusammenarbeiten lässt, die Vorlaufforschung auf bestimmten Gebieten leisten. ${ }^{19}$

Insgesamt kann unter Verweis auf generelle Trends im ökonomischen und technologischen Umfeld der privatwirtschaftlichen Aktivität der generelle Bedeutungszugewinn der arbeitsteiligen Forschungs- und Entwicklungstätigkeit begründet werden. Allerdings reicht dieser Verweis auf generelle Trends nicht aus, um zu erklären, a) welche Teile der internen FuE-Aktivität über den Markt abgewickelt werden oder b) warum der Vertragsforschung im Vergleich zur verti-

(2003), S. 5.

15 Vgl. Chatterji (1996), S. 48, Radnor (1991), S. 118, Howells (1997), S. 10, Veugelers/Cassiman (1999), S. 63, Kulicke/Broß/Gundrum (1997), S. 82, Gauglitz-Lüter (1998), S. 105.

${ }^{16}$ Howells (1997), S. 9. Für Schätzungen der zeitlichen Entwicklung der Durchschnittskosten für Erforschung und Entwicklung neuer Medikamente vgl. Eichin (1995), S. 70, sowie die dort angegebene Literatur.

17 Vgl. UNCTAD (2005), S. 171, Radnor (1991), S. 118 f., Howells (1997), S. 9, De Meuse/Lounsbury/Gordon (1981), S. 30, Kulicke/Broß/Gundrum (1997), S. 82, Chung (1998), S. 42, Koschatzky/Reinhard/Grenzmann (2003), S. 149.

$18 \mathrm{Vgl}$. Kulicke/Broß/Gundrum (1997), S. 82.

19 Vgl. Radnor (1991), S. 119, Howells (1997), S. 10, Koschatzky/Reinhard/Grenzmann (2003), S. 150. 
kalen Integration (Übernahme dritter Unternehmen mit FuE-Kapazitäten) der Vorzug gegeben wird. Diese Fragen sollen in der vorliegenden Arbeit verstärkt untersucht werden.

\subsection{Stand der Forschung}

In der Literatur findet man verschiedene Zugangsweisen zur Auseinandersetzung mit den Vertragsforschungsaktivitäten im Wirtschaftssektor. In den lehrbuchartigen Beiträgen der betriebswirtschaftlichen Literatur zum Innovations-, Technologie- bzw. FuE-Management wird das Phänomen der Vertrags- bzw. Auftragsforschung meist erkannt und als eine Form der externen Wissensbeschaffung von anderen Organisationsformen der betrieblichen FuE-Aktivitäten abgegrenzt. Die Analyse der Problematik der Vertragsforschung beschränkt sich allerdings in diesen Beiträgen meist auf eine Aufzählung von möglichen Vor- und Nachteilen dieser Organisationsform gegenüber anderen Bereitstellungsformen für FuE-Leistungen. ${ }^{20}$

Eine theoretische Auseinandersetzung mit dem Phänomen der Vertragsforschung findet vorwiegend im Rahmen der Entscheidungen über Eigenerstellung oder Fremdbezug statt, wobei zur Erklärung von Integrations- und Desintegrationsentscheidungen häufig die Perspektive des Transaktionskostenansatzes herangezogen wird. ${ }^{21}$ Darüber hinaus geht die betriebswirtschaftliche Forschung der Frage nach der optimalen Ausgestaltung von FuE-Verträgen im Rahmen der Theorie der unvollständigen Verträge nach. ${ }^{22}$

Empirische Untersuchungen der Vertragsforschungsaktivitäten können in vier verschiedene Gruppen eingeteilt werden. Die erste Gruppe bilden Beiträge, die im Auftrag einzelner Länder bzw. der Kommission der Europäischen Gemeinschaften durchgeführt wurden und sich mit der quantitativen Erfassung der Vertragsaktivitäten im FuE-Bereich befassen, sowie Motive der Vertrags-

20 Für einen ausführlichen Literaturnachweis vgl. Rüdiger (2000), S. 5 sowie S. 43-51. $\mathrm{Zu}$ Einzelfragen des Managements von Vertragsforschungsbeziehungen vgl. auch De Meuse/Lounsbury/Gordon (1981), Dohrmann (1982), Leppälä (1995), Gad (2003).

${ }^{21}$ Vgl. Nuhn (1987), Kaltwasser (1994), Schneider/Zieringer (1991), Rüdiger (2000), Dillmann (1996), Dillmann (1997), Tapon/Thong (1997), Tapon (1989), Pisano (1990), Hermes (1995).

22 Vgl. Aghion/Tirole (1994), Adams/Marcu (2004). 
vergabe näher untersuchen. ${ }^{23}$ Dieser Gruppe können auch zwei Beiträge zugerechnet werden, in denen Ergebnisse der Fördermaßnahme „Externe Vertragsforschung" des Bundesministers für Forschung und Technologie bilanziert wurden, sowie die Studie von Rüdiger, in der Rahmen- und Erfolgsbedingungen der Vertragsforschung ostdeutscher FuE-Dienstleister Untersuchungsgegenstand waren. ${ }^{24}$

Die zweite Gruppe bilden Studien der Organisationsstruktur der FuE-Aktivitäten in der pharmazeutischen Industrie, einer Branche, in der Outsorcingstrategien im FuE-Bereich besonders stark verbreitet sind. ${ }^{25}$

Der dritten Gruppe können acht ökonometrische Studien zugeordnet werden. In zwei Beiträgen werden Determinanten der Vertragsforschung untersucht, wobei Hypothesen über die Bestimmungsgründe der Vertragsvergabe primär auf der Grundlage der Analyse von innovationsökonomischer Literatur abgeleitet werden. ${ }^{26}$ In einer Studie wird bei der Analyse der Determinanten der Vertragsforschung auf Erkenntnisse der Transaktionskostentheorie, des Property-Rights-Ansatzes und der vorliegenden empirischen Evidenz verwiesen. ${ }^{27}$ Nur die letzte Studie nimmt dabei eine Schätzung der Determinanten der Durchführung von FuE-Aufträgen vor. Die Befunde dieser Untersuchungen werden in Kapitel 5 den Ergebnissen der vorliegenden eigenen Analyse gegenübergestellt.

In zwei weiteren Beiträgen der dritten Gruppe wird der Frage nachgegangen, warum interne FuE der Vertragsforschung vorgezogen wird. ${ }^{28}$ Eine Studie überprüft, ob Vertragsforschung komplemetär oder substitutiv zur internen FuE eingesetzt wird. ${ }^{29}$ Ein Beitrag befasst sich mit der Frage nach den Determinanten der Fremdvergabe von klinischen Studien durch Unternehmen der Pharmaindustrie..$^{30}$ In der letzten Studie dieser Gruppe wird die Beziehung zwi-

23 Vgl. Röthlingshöfer (1972), Kommission der Europäischen Gemeinschaften (1973), Traill/Miege (1989), Reiß/Hüsing (1992), Ringe (1991), Koschatzky/Reinhard/Grenzmann (2003).

24 Vgl. Allesch/Fiedler/Martin (1983), Fraunhofer ISI/Prognos AG (1991), Rüdiger (2000).

25 Vgl. Pisano (1991), Dillmann (1996, 1997), Tapon (1989), Tapon/Cadsby (1996), van den Bosch/Elfferich (1991), Zumbroich/Gadicke/Steiner (1995), Rothwell/Dodgson (1991).

26 Vgl. den Hertog und Thurik (1993), Leiponen (2002).

27 Vgl. Adams/Marcu (2004).

28 Vgl. Beneito (2003), Pisano (1990).

$29 \mathrm{Vgl}$. Veugelers (1997).

30 Vgl. Azoulay (2003). 
schen den gewählten Koordinationsformen für FuE-Leistungen (interne FuE, Vertragsforschung und Kombination beider Formen) und dem FuE-Output untersucht. $^{31}$

Keine dieser ökonometrischen Studien verwendet jedoch Daten zur FuE-Tätigkeit deutscher Unternehmen.

Die letzte Gruppe bilden einige Beiträge, die keiner der zuvor erwähnten Gruppen zugerechnet werden können. In einer Studie wird unter Zuhilfenahme von Archivdaten der Frage nach der Rolle der Vertragsforschung für US-amerikanische Unternehmen des Verarbeitenden Gewerbes im Zeitraum zwischen 1900 und 1940 nachgegangen. ${ }^{32}$ In einer auf Fallstudien basierten Untersuchung werden Motive der Vertragsvergabe systematisiert. ${ }^{33}$ Mehrere Beiträge widmen sich schließlich der Charakterisierung einzelner Vertragsforschungsanbieter. ${ }^{34}$

Darüber hinaus kann auch auf einige Studien hingewiesen werden, die sich nicht explizit mit dem Phänomen der Vertragsforschung beschäftigen, sondern der allgemeiner gehaltenen Frage nach den Bestimmungsfaktoren der „externen Technologiebeschaffung " (external technology acquisition ${ }^{35}$ ) nachgehen. In diesen Studien wird Vertragsforschung als eine der möglichen unternehmensexternen Koordinationsformen für FuE-Aktivitäten mit erfasst, ohne dass eine eingehende Analyse der Besonderheiten der Vertragsforschung in Abgrenzung zu Kooperationsbeziehungen bzw. Lizenzverträgen erfolgt. ${ }^{36}$

Wie aus der Systematisierung der vorliegenden Literatur hervorgeht, liegen bisher nur partielle Erkenntnisse zu den Bestimmungsgründen der Vertragsforschung vor. Zum einen wird in der theoretischen Literatur der Frage nach der optimalen Gestaltung von FuE-Verträgen nachgegangen, ohne dass eine Erklärung für die grundlegende Entscheidung für Vertragsforschung geboten wird. Empirische Untersuchungen der Vertragsforschungsaktivitäten gehen zudem meist über eine generelle Bestandsaufnahme, Fallstudien bzw. die reine

$31 \mathrm{Vgl}$. Beneito (2006).

32 Vgl. Mowery (1983). Eine Aufarbeitung der Geschichte der Vertragsforschung in Deutschland und den Vereinigten Staaten liefert darüber hinaus Lieske (2000).

${ }^{33}$ Vgl. Haour (1992).

$34 \mathrm{Vgl}$. insbesondere die Literaturhinweise in Rüdiger (2000), S. 6.

35 Vgl. Granstrand/Bohlin/Oskarsson/Sjöberg (1992).

${ }^{36}$ Vgl. Veugelers/Cassiman (1999), Kurokawa (1997), Croisier (1998), Nagarajan/Mitchell (1998), Narula (2001), Chatterji (1996), Granstrand/Bohlin/Oskarsson/Sjöberg (1992). 
Motivforschung nicht hinaus. Der Forschungsstand hinsichtlich des zu untersuchenden Phänomens weist folglich erhebliche Defizite auf.

An dieser Stelle setzt die vorliegende Arbeit an. Auf der Basis einer theoriegeleiteten Auseinandersetzung mit dem Phänomen der Vertragsforschung sollen Bestimmungsfaktoren der Vertragsforschung abgeleitet und anhand von Daten zur Forschungstätigkeit deutscher Unternehmen einer empirischen Prüfung unterzogen werden. Somit wird in der vorliegenden Arbeit zum ersten Mal die Schätzung der Bestimmungsfaktoren der Vertragsforschung in der Population der deutschen Unternehmen vorgenommen.

Der Fall Deutschlands ist aus drei Gründen von besonderem Interesse. Erstens zählt Deutschland zu den forschungsintensivsten Ländern der Welt; im Jahre 2005 wurden $6 \%$ aller weltweiten FuE-Ausgaben in Deutschland getätigt. ${ }^{37} \mathrm{Im}$ Vergleich zu Finnland, den Vereinigten Staaten und den Niederlanden - d.h. Ländern, für die die bisherigen ökonometrischen Untersuchungen von Bestimmungsfaktoren der Vertragsforschung vorliegen - ist die deutsche Wirtschaft jedoch viel stärker auf die forschungsintensive Fertigungstechnik ausgerichtet, die etwa $60 \%$ der FuE-Aufwendungen auf sich vereint. ${ }^{38}$ Zweitens ist Deutschland traditionell stark exportorientiert und war 2005 für 16,5\% aller OECDExporte an Technologiegütern verantwortlich. Dies macht Deutschland zum exportstärksten Land von Technologiegütern. ${ }^{39}$ Drittens zeigt die Studie von Lieske (2000), dass sich das Instrument der Vertragsforschung in Deutschland - im Vergleich zu den Vereinigten Staaten - erst spät etabliert hat. Zugleich weisen die vorliegenden Daten der Wissenschaftsstatistik (vgl. Abb. 1.1) auf einen erheblichen Bedeutungszugewinn dieser Koordinationsform im Verlauf der letzten 20 Jahre hin, so dass eine eingehende Analyse der Gründe für die mittlerweile weit verbreitete Nutzung dieses Instruments geboten erscheint.

\subsection{Aufbau der Arbeit und Gang der Unter- suchung}

Die Arbeit gliedert sich in sechs Kapitel. Im Anschluss an diese Einleitung sollen in Kapitel 2 zunächst definitorische Grundlagen der vorliegenden Stu-

$37 \mathrm{Vgl}$. Bundesministerium für Bildung und Forschung (2006), S. 1.

38 Vgl. Legler/Krawczyk (2006), Abb. 2.4.1, S. 55, Bundesministerium für Bildung und Forschung (2006), S. 5.

$39 \mathrm{Vgl}$. Bundesministerium für Bildung und Forschung (2006), S. 1. 
die formuliert werden. Im ersten Schritt wird dabei die Forschungs- und Entwicklungstätigkeit definiert und von der - breiter verstandenen - Innovationstätigkeit abgegrenzt. Im zweiten Schritt erfolgt die Festlegung des für die Zwecke der vorliegenden Arbeit gültigen Begriffs der Vertragsforschung. Die zu wählende Definition soll sowohl eine theoretische Analyse des Phänomens der externen Beschaffung von FuE-Dienstleistungen ermöglichen, als auch die statistische Erfassbarkeit dieses Phänomens gewährleisten. Dazu wird bei der definitorischen Abgrenzung der Vertragsforschung sowohl auf die institutionenökonomisch geprägten Systematisierungsansätze, als auch auf die im sogenannten Frascati-Manual ${ }^{40}$ formulierten Richtlinien zur Erhebung der Daten zur Forschungs- und Entwicklungstätigkeit eingegangen. Im letzten Schritt erfolgt die Diskussion der in der Literatur vorzufindenden Konzepte von „Wissen". Dies erscheint notwendig, weil neues technologisches Wissen als Ergebnis von FuE-Tätigkeit das eigentliche Transaktionsobjekt bei den FuEVertragsbeziehungen darstellt.

Das 3. Kapitel fasst die Ergebnisse neuester theoretischer wie empirischer Studien zu den Determinanten der Forschungs- und Entwicklungstätigkeit im Wirtschaftssektor zusammen. Dies erscheint notwendig, da - wie im letzten Abschnitt ausgeführt - bei der Ableitung der Hypothesen über die Bestimmungsgründe der Vertragsforschung auf vorliegende Erkenntnisse über allgemeine Determinanten der FuE-Aktivitäten im Wirtschaftssektor angeknüpft wird. Die in diesem Kapitel erfolgende Aufarbeitung der einschlägigen Literatur ermöglicht es, grundsätzliche Überlegungen zur Natur der Forschungstätigkeit im Wirtschaftssektor anzustellen und somit die relevanten Bausteine jeglicher Auseinandersetzung mit diesem Phänomen zu identifizieren. Die systematische und konsequente Darstellung der Methodologie der empirischen Studien bietet darüber hinaus die Gelegenheit, die bereits angesammelten Erfahrungen hinsichtlich der Operationalisierung bestimmter Konzepte zu analysieren und als Ausgangspunkt der empirischen Untersuchung von Determinanten der Vertragsforschung im 5. Kapitel der Arbeit zu verwenden.

Das 4. Kapitel der Arbeit ist der theoretischen Auseinandersetzung mit dem Phänomen der Vertragsforschung gewidmet. Da jedoch eine distinktive Theorie der Vertragsforschung in der einschlägigen Literatur bislang fehlt, gilt es

40 Beim Frascati-Manual handelt es sich um ein Handbuch der Organisation für wirtschaftliche Zusammenarbeit und Entwicklung (OECD), in welchem Richtlinien zur Messung wissenschaftlicher und technischer Tätigkeiten festgelegt wurden. Die in diesem Handbuch empfohlenen Definitionen bilden die Grundlage für empirische Erhebungen zu den FuEAufwendungen in einzelnen Mitgliedstaaten der OECD. 
die bestehenden wirtschaftswissenschaftlichen Theorien und Modelle auf ihre Eignung für die Erklärung des Phänomens der arbeitsteiligen FuE-Prozesse zu prüfen. Kriterien zur Auswahl relevanter theoretischer Ansätze werden in Abschnitt 4.1 formuliert. Anschließend erfolgt die Diskussion der Grundstruktur der ausgewählten Theorierichtungen sowie eine Übertragung der zentralen Konzepte auf den Kontext der Vertragsforschung (Abschnitte 4.2-4.4). Im Mittelpunkt der Analyse steht dabei die Ableitung empirisch gehaltvoller $\mathrm{Hy}-$ pothesen über die Bestimmungsfaktoren der Vertragsforschung. Die Zusammenführung und Strukturierung der Hypothesen in Abschnitt 4.5 bilden die Grundlage für den empirischen Teil der Arbeit.

In Kapitel 5 werden die formulierten Hypothesen über die Bestimmungsfaktoren der Vertragsforschung einer empirischen Prüfung unterzogen. Die Datenbasis für die empirische Studie bildet der Datensatz des Mannheimer Innovationspanels, einer deutschen Teilstudie im Rahmen der von EUROSTAT koordinierten europaweiten Innovationserhebungen (Community Innovation Surveys, CIS). Dieser Datensatz erlaubt es, sowohl die Perspektive der Auftraggeber als auch die der FuE-Dienstleister zu analysieren. Somit können in dieser Arbeit Erkenntnisse über die privatwirtschaftlichen Anbieter und Nachfrager der FuEDienstleistungen gewonnen werden.

Kapitel 6 schließt die Arbeit mit einer zusammenfassenden Darstellung der zentralen Ergebnisse ab. 


\section{Kapitel 2}

\section{Grundlegende Begriffe, Abgrenzungen und Klassifikationen}

In diesem Kapitel sollen die definitorischen Grundlagen der Arbeit ausformuliert werden. In Abschnitt 2.1 wird zunächst die Forschungs- und Entwicklungstätigkeit in Übereinstimmung mit den Vorgaben des Frascati-Handbuchs definiert. Es erfolgt eine Aufzählung der konstitutiven Merkmale der FuETätigkeit, eine Erläuterung ihrer Systematisierungsmöglichkeiten und ihre Einordnung als ein Bestandteil des Innovationsprozesses. In Abschnitt 2.2 wird die für die vorliegende Untersuchung geltende Definition der Vertragsforschung festgelegt. Bei der Herleitung der Definition steht zunächst die Abgrenzung der Vertragsforschung von anderen Formen der Organisation von FuE-Prozessen im Vordergrund der Analyse. Anschließend werden die im Frascati-Manual formulierten Richtlinien zur Erhebung der Daten zur Forschungs- und Entwicklungstätigkeit erläutert. Es soll gezeigt werden, auf welche Weise das Phänomen der Vertragsforschung statistisch erfasst werden kann. Eine Gegenüberstellung der theoretischen Systematisierungsansätze und der empirischen Erhebungspraxis ermöglicht eine konsistente Abgrenzung des Untersuchungsgegenstandes. Im letzten Abschnitt 2.3 erfolgt die Diskussion der in der Literatur vorzufindenden Konzepte von „Wissen“ als ökonomischem Gut. Diese Diskussion ist notwendig, weil neues innovationsrelevantes Wissen als Ergebnis der FuETätigkeit das eigentliche Transaktionsobjekt bei den FuE-Vertragsbeziehungen darstellt. 


\subsection{Zum Begriff Forschung und Entwicklung}

Während in der einschlägigen Literatur der Begriff Forschung und Entwicklung (FuE) immer noch unterschiedlich abgegrenzt wird, hat die OECD im sog. „Frascati Manual“ bereits in den 60er Jahren eine Empfehlung hinsichtlich der Definition und Abgrenzung dieser Tätigkeit angegeben. ${ }^{41} \mathrm{Da}$ dieses Handbuch Richtlinien zur Messung wissenschaftlicher und technischer Tätigkeiten festlegt und somit die Grundlage für die empirischen Erhebungen zu den FuE-Aufwendungen in einzelnen Mitgliedstaaten der OECD, darunter auch in Deutschland, bildet, erscheint es sinnvoll, in den nachfolgenden Ausführungen von der dort vorgeschlagenen begrifflichen Bestimmung auszugehen.

Im Rahmen des Frascati Manuals wird Forschung und Entwicklung als „systematische, schöpferische Arbeit zur Erweiterung des vorhandenen Wissens und die Anwendung dieses Wissens auf neue Probleme" definiert. ${ }^{42}$ Im Sinne dieser Definition umfasst FuE eine geplante, strukturiert ablaufende Suche nach neuen Erkenntnissen technisch-naturwissenschaftlicher und sozioökonomischer Art. Da bei dieser Suche Ressourcen eingesetzt werden, deren neuartige Kombinationen Generierung neuen Wissens ermöglichen soll, wird FuE oft als eine besondere Art von Produktionsprozessen betrachtet. ${ }^{43}$ Spezifische Merkmale der FuE-Tätigkeit ermöglichen die Abgrenzung der FuE von herkömmlichen Faktorkombinationsprozessen und sollen daher nachfolgend näher erläutert werden.

\subsubsection{Merkmale von Forschungs- und Entwicklungsak- tivitäten}

Definitionsgemäß handelt es sich bei FuE um einen Vorgang, bei dem neues Wissen produziert wird. Das Ergebnis dieser Tätigkeit - auch als Invention bezeichnet - ist also primär immaterieller Natur und umfasst lediglich die Konzeption einer Neuheit. ${ }^{44}$ Neuheit kann in diesem Zusammenhang sowohl

41 Zur Geschichte des Frascati-Handbuchs vgl. OECD (2002), S. Anhang 1, S. 151-156. Für eine deutschsprachige Fassung des Frascati-Handbuchs vgl. Bundesminister für Forschung und Technologie (1982).

42 Vgl. OECD (2002), S. 30.

${ }^{43}$ Zur faktorkombinationsorientierten Auffassung der FuE-Prozesse vgl. Brockhoff (1999), S. 48, Corsten (1998), S. 151, sowie Arrow (1962), S. 609.

44 Vgl. Gauglitz-Lüter (1998), S. 15. 
subjektiv als auch objektiv aufgefasst werden. ${ }^{45}$ Von der objektiven Neuheit wird gesprochen, wenn es sich bei der Invention um eine bisher weltweit nicht existierende Problemlösung handelt. Subjektiv betrachtet bezieht sich das Attribut "neu“ auf den Kenntnisstand der jeweiligen Unternehmung. Die letztere, also eine weiter gefasste Interpretation wird in dieser Arbeit zugrunde gelegt, weil sie die Berücksichtigung auch jener Aktivitäten ermöglicht, mit denen die Erforschung des bereits im technologischen Umfeld einer Unternehmung vorhandenen Wissens bezweckt wird.

Zweitens handelt es sich bei Forschung und Entwicklung definitionsgemäß um eine schöpferische Arbeit, was impliziert, daß unter den in diesem Faktorkombinationsprozess eingesetzten Ressourcen dem Humankapital eine besondere Rolle zukommt. Neben der menschlich kreativen Arbeit fließt auch das bereits vorhandene Wissen in die FuE-Tätigkeit ein. ${ }^{46}$ Die Hervorhebung der Ressource Wissen als einen der zentralen Input- und Outputfaktoren dieser Tätigkeit verdeutlicht zudem den kumulativen Charakter der FuE-Tätigkeit. ${ }^{47}$

$\mathrm{Zu}$ den distinktiven Eigenschaften der Forschung und Entwicklung gehört auch die interne und externe Unsicherheit. ${ }^{48}$ Die interne Unsicherheit erstreckt sich auf drei Teilbereiche. ${ }^{49}$ Erstens ist die Input-Output-Relation in FuE nicht deterministisch; der Faktoreinsatz führt nicht zwangsläufig zu den angestrebten Ergebnissen. ${ }^{50}$ Diese erste Art der internen Unsicherheit, die auch als generelle Ergebnisunsicherheit ${ }^{51}$ bezeichnet wird, betrifft folglich die Frage, ob für das gestellte Problem bei gegebenen Kapazitäten und vorhandenem Wissensstand der Organisation eine realisierbare Lösung gefunden werden kann. Zweitens besteht interne Unsicherheit in Bezug auf die Realisierungszeiten. Drittens sind die FuE-Aktivitäten durch Kostenunsicherheiten gekennzeichnet, die sich

45 Vgl. Kern/Schröder (1977), S. 15, Specht/Beckmann (1996), S. 16.

$46 \mathrm{Vgl}$. dazu Arrow (1962), S. 618: „Information is not only the product of inventive activity, it is also an input - in some sense, the major input apart from the talent of the inventor."

47, "...] the acquisistion of knowledge is cumulative and interactive: knowledge itself is an important input into the production of knowledge", Arora (1995), S. 162.

48 Vgl. Arrow (1962), S. 616, Gauglitz-Lüter (1998), S. 16, Schneider/Zieringer (1991), S. 8, Hansen (1999), S. 34 ff. Kern und Schröder stellen die Unsicherheit als konstitutive Eigenschaft der Forschung und Entwicklung hervor: „Ist das Ergebnis eines Vorhabens a priori sicher, so handelt es sich nicht um Forschung und Entwicklung “, Kern/Schröder (1977), S. 27.

49 Zur alternativen, vertieften Gliederung vgl. Dilling (2002), S. 31-40.

$50 \mathrm{Vgl}$. Brockhoff (1999), S. 49, Corsten (1998), S. 151.

51 Vgl. Gauglitz-Lüter (1998), S. 16. 
aus der Schwierigkeit ergeben, den nötigen Ressourcenverbrauch genau abzuschätzen bzw. zu bestimmen. Die externe Unsicherheit besteht im Hinblick auf die Verwertungsmöglichkeiten der erzielten Inventionen, da das Nachfragepotenzial und die Marktentwicklung nur ungenau antizipiert werden können. ${ }^{52}$

Viertens ist die Durchführung von Forschungsprojekten in der Regel mit beträchtlichen versunkenen Kosten verbunden, wobei sunk costs als nicht rückholbare Kosten der vorzeitig abgebrochenen Investitionsprojekte definiert werden können. ${ }^{53}$ Die FuE-Aufwendungen weisen die Eigenschaften der versunkenen Kosten insofern auf, als bei eingestellten Projekten selten ein Liquidationswert vorliegt. ${ }^{54}$ Exemplarisch kann das anhand von Investitionen in Humankapital verdeutlicht werden. Die für die Abwicklung eines Forschungsprojektes benötigten Ausgaben für Qualifizierung und Weiterbildung der beteiligten Mitarbeiter sind ihrer Natur nach irreversibel; die getätigten Auslagen können nicht zurückerlangt werden. ${ }^{55}$

Charakteristisch für die Produktion des Gutes Wissen ist auch das Vorliegen des Unteilbarkeitsproblems. Es wird darauf hingewiesen, dass technische Gegebenheiten einen mindestoptimalen Umfang für die Forschungstätigkeit verlangen. ${ }^{56}$ Da einzelne FuE-Projekte den Kauf oder die Entwicklung teurer Spezialgeräte, Mess- und Testverfahren erfordern, erscheint es sinnvoll, ihre Auslastung durch mehrere Projekte zu gewährleisten, um die durchschnittlichen Kosten eines Projekts zu senken.

Zusammenfassend kann festgehalten werden, dass Forschung und Entwicklung aus faktorkombinationsorientierter Betrachtungsweise einen kumulativen Produktionsprozess darstellt, der durch einen immateriellen Ergebnischarakter, erhebliche interne und externe Unsicherheiten sowie durch die besondere Rolle der versunkenen Kosten und Unteilbarkeiten gekennzeichnet ist.

52 Vgl. Dilling (2002), S. 33 f.

${ }^{53} \mathrm{Zu}$ dieser Definition der versunkenen Kosten vgl. Chung (1998), S. 193, zur allgemeinen Abhandlung der Problematik der versunkenen Kosten vgl. insbesondere Krahnen (1991), S. 21-69.

54 Vgl. Chung (1998), S. 193 f., Dilling (2002), S. 40 f.

55 Vgl. Stiglitz (1987), S. 928, Hansen (1999), S. 36. Allerdings weist Chung darauf hin, dass „abgebrochene FuE-Prozesse wertvolles Wissen zur weiteren internen Verwendung generieren", Chung (1998), S. 194.

56 Vgl. Fritsch (1993), S. 6, Foray (1995), S. 80, Czarnitzki (2004), S. 5. 


\subsubsection{Klassifikationsmöglichkeiten}

Nachfolgend werden zwei Klassifikationsmöglichkeiten erläutert, welche die Vielfalt der unter dem Begriff Forschung und Entwicklung subsummierten Teilaktivitäten systematisch erfassen.

Anhand des ersten Kriteriums Anwendungsbezug können drei FuE-Arten unterschieden werden: Grundlagenforschung, angewandte Forschung und experimentelle Entwicklung, die in der vorliegenden Arbeit - in Übereinstimmung mit den Vorgaben des Frascati-Manuals - folgendermaßen abgegrenzt werden: ${ }^{57}$

Grundlagenforschung ist experimentelle oder theoretische Arbeit, die in erster Linie auf die Gewinnung neuer Erkenntnisse über den zugrunde liegenden Ursprung von Phänomenen und beobachtbaren Tatsachen gerichtet ist, ohne auf eine besondere Anwendung oder Verwendung abzuzielen.

Angewandte Forschung umfasst alle Anstrengungen, die auf die Gewinnung neuer Erkenntnisse gerichtet sind. Sie ist jedoch in erster Linie auf ein spezifisches, praktisches Ziel oder eine bestimmte Zielsetzung gerichtet.

Experimentelle Entwicklung ist systematische, auf vorhandenen Erkenntnissen aus Forschung und/oder praktischer Erfahrung aufbauende Arbeit, die auf die Herstellung neuer Materialien, Produkte und Geräte und die Einführung neuer Verfahren, Systeme und Dienstleistungen sowie deren wesentliche Verbesserung abzielt.

Diese Systematisierung der unter dem Begriff Forschung und Entwicklung subsummierten Teilaktivitäten wird auch als phasenbezogene Gliederung aufgefasst. Demnach wird der Wissensgenerierung ein linearer Ablauf unterstellt: auf der Basis des in der Grundlagenforschung erarbeiteten allgemeinen Wissenspotenzials wird bei zunehmend detaillierten Zielvorgaben immer anwendungsorientierter und marktnäher geforscht. ${ }^{58}$ Diese Auffassung führt jedoch zu kritikfähigen Implikationen. Insbesondere wird bei der Voraussetzung der Linearität sowohl die Induktion der Forschung und Entwicklung durch die Nachfrage, als auch die Möglichkeit von Rückkopplungen im Forschungsprozess ausgeschlossen. Daher wird in dieser Arbeit der sequentielle Ablauf der

57 Vgl. Bundesminister für Forschung und Technologie (1982), S. $70 \mathrm{f}$.

58 Vgl. Ehlken (1999), S. 7, Dilling (2002), S. 9. 
FuE-Tätigkeit nicht unterstellt. Die Einteilung in Grundlagenforschung, angewandte Forschung und experimentelle Entwicklung dient vorwiegend der Hervorhebung der Unterschiede bezüglich der Unsicherheit und der Verwertungsmöglichkeiten der erarbeiteten Ergebnisse. ${ }^{59}$ Als konsensfähig gilt, dass mit der zunehmenden Anwendungsorientierung der Konkretisierungsgrad der Zielformulierung sowie die Entrittswahrscheinlichkeit der Ergebnisse steigen und das Ausmaß der technisch bedingten (internen) Unsicherheit abnimmt. ${ }^{60}$ Die steigende Praxisbezogenheit bewirkt zugleich, dass die Ergebnisse der angewandten Forschung als Antworten auf konkrete Problemstellungen einen weniger universellen Charakter aufweisen.

Anhand des Kriteriums der Zielsetzung der FuE-Tätigkeit kann ferner zwischen Produkt- und Prozess-FuE unterschieden werden. Dabei gilt es jedoch zu beachten, dass diese Einteilung aus der Perspektive der FuE-durchführenden Einheit vorgenommen wird und keine absolute Trennschärfe aufweist. So können die Unternehmen die Erforschung einer Verfahrensneuheit sowohl als Prozess-FuE als auch als Produkt-FuE auffassen, beispielsweise wenn neuartige Verfahren zur „Produktpalette“ des Unternehmens gehören.

\subsubsection{Forschung und Entwicklung im Innovationspro- zess}

Neben der bereits vorgestellten faktorkombinationsorientierten Betrachtungsweise findet man in der einschlägigen Literatur eine weitere Konzeption von FuE, die diese Tätigkeit als die erste Phase eines Innovationsprozesses auffasst. Das innovationstheoretische Phasenmodell wird in diesem Abschnitt näher erläutert, weil es erlaubt, Forschung und Entwicklung von anderen wissenschaftlichen und technischen Aktivitäten abzugrenzen sowie die definitorischen Unterschiede zwischen FuE- und Innovationstätigkeit herauszustellen.

Im Rahmen des Phasenmodells des Innovationsprozesses wird der Vorgang der Entstehung und Verbreitung einer wirtschaftlichen Neuerung in idealtypische Teilaktivitäten unterteilt. In Anlehnung an die von Schumpeter gegründete Tradition werden hierbei meistens drei Phasen unterschieden. ${ }^{61}$ Die erste Phase, bei der das Erkennen eines Problems bzw. einer Wissenslücke und die Ent-

59 Vgl. Björn (2002), S. 27, Brockhoff (1999), S. 55.

60 Vgl. Hansen (1999), S. 35, Ehlken (1999), S. 7 f., Goel/Rich (2005), S. 3, Nelson (1959), S. 300.

61 Vgl. Ehlken (1999), S. 16. 
wicklung der Konzeption einer technisch realisierbaren Lösung dieses Problems im Mittelpunkt steht, wird als Inventionsphase bezeichnet und ist der FuETätigkeit im Sinne der dargestellten, faktorkombinationsorientierten Definition äquivalent. Wird die Invention als Ergebnis dieser Teilphase als wirtschaftlich verwertbar eingestuft, erfolgt die Umsetzung des neu gewonnen Wissens in ein neues Produkt bzw. Verfahren. Die Aktivitäten von der erstmaligen Umsetzung bis hin zur Markteinführung der Neuheit werden der Innovationsphase zugerechnet. ${ }^{62}$ Der Markteinführung ist die Phase der Diffusion, d.h. der räumlichen und zeitlichen Verbreitung einer wirtschaftlichen Neuheit, nachgelagert.

Eine scharfe Abgrenzung zwischen den einzelnen Phasen erweist sich in der Praxis als problembehaftet und erfolgt aus diesem Grunde oft über die Auflistung von Grenzfällen. ${ }^{63}$ Beispielweise wird der Übergang zwischen der Inventions- und Innovationsphase im Bereich der Ingenieur- und Technologiewissenschaften durch den Prototypenbau markiert. ${ }^{64}$ Diese Tätigkeit wird der FuE-Phase nur dann zugeordnet, wenn das Hauptziel in der Erarbeitung weiterer Verbesserungen liegt. Dient die Erstellung und Erprobung des Prototyps der Ingangsetzung des Produktionsprozesses, zählen die dafür getätigten Aufwendungen nicht zu den FuE-, sondern zu den Innovationsausgaben.

In der ergebnisorientierten Sichtweise kann folglich zwischen der FuE-Phase mit dem Ergebnis der Invention und der Innovationsphase mit dem Ergebnis der Innovation unterschieden werden. Die prozessorientierte Sichtweise bezieht dagegen den gesamten, auf das Hervorbringen einer wirtschaftlichen Neuerung abzielenden Vorgang in das Verständnis der Innovationstätigkeit ein. ${ }^{65}$ In diesem Sinne wird in der vorliegenden Arbeit die Forschungs- und Entwicklungstätigkeit als ein Produktionsvorgang aufgefasst, der auf die Erarbeitung neuen technologischen Wissens abzielt und einen Bestandteil der breiter verstandenen Innovationstätigkeit der Wirtschaftsakteure darstellt. Als innovierende Unternehmen werden folglich diejenigen Wirtschaftseinheiten aufgefasst, die Tätigkeiten aufnehmen, die sowohl der Inventions- als auch der Innovationsphase zugerechnet werden können.

62 Stiglitz fasst den Unterschied zwischen den beiden Phasen folgendermaßen auf: „Forschung kann als ein Prozess der Informationsgewinnung angesehen werden und Innovation als Prozess der Übersetzung von Ideen, also ebenfalls Informationen, in neue Produkte“, Stiglitz (1999), S. 180.

63 Vgl. dazu Tabelle 2.3. in OECD (2002), S. 41. bzw. Stifterverband für die Deutsche Wissenschaft (2001), Anhang 3, S. 40*.

64 Vgl. Klodt (1995), S. 120.

65 Vgl. Specht/Beckmann (1996), S. 15. 
Schließlich sei noch angemerkt, dass das oben dargelegte sequentielle Innovationsmodell bereits seit den 50er Jahren einer Kritik unterzogen wurde, die zu einer Reihe von Verbesserungsvorschlägen führte. Spätere Modelle des Innovationsprozesses haben die Bedeutung der Nachfrageseite, der Rückkopplungen zu vorgelagerten Phasen sowie der Interaktionen zwischen verschiedenen Unternehmenseinheiten (Forschung und Entwicklung, Produktion, Marketing) und mit anderen Marktteilnehmern hervorgehoben.$^{66}$ Die durch die neuen Modelle eingebrachten Verbesserungen haben jedoch die Gültigkeit der Schumpeterschen Erkenntnisse über die Grundstruktur des Innovationsprozesses nicht aufgehoben ${ }^{67}$, sondern ermöglichten vielmehr eine vertiefte Analyse des Phänomens der Innovationstätigkeit.

\subsection{Einordnung und Abgrenzung der Ver- tragsforschung}

Wesentlich für die Begriffsbestimmung ist zunächst die Abgrenzung der Vertragsforschung von anderen Formen der Koordination von FuE-Aktivitäten. Aus einzelwirtschaftlicher Perspektive bestehen grundsätzlich drei Alternativen zur Beschaffung neuen innovationsrelevanten Wissens:

- eine unternehmensinterne Forschungs- und Entwicklungstätigkeit,

- Bereitstellung der FuE-Leistung in Kooperation mit einem bzw. mehreren Akteuren (Unternehmen, staatlichen Institutionen, Hochschulinstituten) und

- Beschaffung neuen Wissens von einem unternehmensexternen Partner.

Ausgehend von dieser Dreiteilung haben Schneider und Zieringer (1991) eine Typologie vorgeschlagen, welche die Erscheinungsformen der Organisation der FuE-Tätigkeit in das Spektrum zwischen Markt und Hierarchie einordnen läßt

66 Vgl. z.B. die Modelle von Kline/Rosenberg (1986), S. 290, Schmidt-Tiedemann (1982), S. 20; zur systematischen und chronologischen Aufarbeitung der Theorien der Innovationsprozesse vgl. insbesondere Hipp (2000), S. 25-51.

${ }^{67} \mathrm{Zu}$ dieser Einschätzung vgl. z.B. Hipp (2000), S. 32. 
Bereitstellungsformen für die Durchführung von FuE-Leistungen

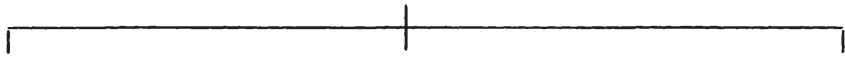

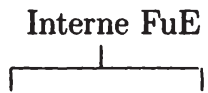

Unternehmens- Übernahme interne FuE von FuE Abteilung durch Akquisition

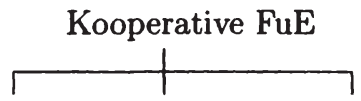

Gemein- Koordinierte Erfahrungs-, schafts- Einzel- ErgebnisFuE austausch

\section{Externe FuE}

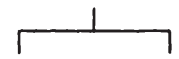

Vertrags-, LizenzAuftrags- nahme FuE

Quelle: Schneider /Zieringer (1991), S. 47.

(vgl. Abb. 2.1). ${ }^{68}$ Anhand dieses Systematisierungsansatzes sollen nachfolgend die einzelnen Alternativen dargelegt und voneinander abgegrenzt werden.

$\mathrm{Zu}$ den Gestaltungsformen der internen FuE (hierfür wird auch der Ausdruck Eigenforschung verwendet) zählen im Systematisierungsansatz von Schneider und Zieringer die unternehmensinterne Abwicklung in organisatorisch selbstständigen FuE-Abteilungen sowie die Übernahme fremder Unternehmen mit einer FuE-Abteilung. ${ }^{69}$ Bei der zweiten Alternative handelt es sich um eine Integrationslösung in dem Sinne, dass bei der FuE-Tätigkeit eine Unternehmung auf Ressourcen zurückgreifen kann, an denen sie exklusive Nutzungsbzw. Eigentumsrechte hält. ${ }^{70}$ Einschränkend in der Typologie von Schneider und Zieringer (1991) wirkt die Voraussetzung, dass FuE-Tätigkeit in formalisierter Form, d.h. in einer FuE-Abteilung stattfinden muss. Diese Annahme erscheint unnötig eng, wenn bedacht wird, dass innerbetriebliche FuE-Prozesse auch außerhalb einer FuE-Abteilung stattfinden können. ${ }^{71}$

68 Für alternative Systematisierungsvorschläge vgl. Croisier (1998), S. 292, Dillmann (1996), S. 41, Tapon (1989), S. 198, Foray (1991), S. 402, Granstrand/Bohlin/Oskarsson/Sjöberg (1992), S. 113.

69 Vgl. Schneider/Zieringer (1991), S. 26.

70 Vgl. Chung (1997), S. 29.

71 Die empirischen Studien zeigen beispielweise, dass lediglich $45,3 \%$ der forschenden Klein- und Mittelunternehmen (mit bis zu 250 Beschäftigten) über eine FuE-Abteilung verfügen; viele Forschungsprojekte werden in Klein- und Mittelunternehmen (KMUs) bei Abwesenheit der FuE-Abteilung durchgeführt, vgl. dazu Harhoff/Licht (1996), S. 25. 
Als Abwicklungsalternativen für die unternehmensexterne FuE (Fremdforschung) werden Vertrags- bzw. Auftragsforschung sowie Lizenznahme genannt. Bei der ersten Gestaltungsform handelt es sich nach dem Definitionsvorschlag von Schneider und Zieringer um einen Vorgang, bei dem außenstehende Dritte gegen Vergütung ein festgelegtes FuE-Programm übernehmen und die gewonnen Erkenntnisse dem Auftraggeber zur Verfügung stellen. ${ }^{72}$ Als Auftragnehmer können dabei nicht nur andere Unternehmen, sondern auch öffentliche Einrichtungen aus dem In- und Ausland in Erscheinung treten. Die Lizenznahme stellt dagegen einen „Extremfall der externen FuE“ insofern dar, als hierbei lediglich das bereits auf dem Beschaffungsmarkt zugängliche immaterielle Produkt zugekauft wird. Bei dem Produkt kann es sich um „Erfindungen" sowie Know-how im weitesten Sinne handeln. ${ }^{73}$ Bei der Abgrenzung von Auftrags- bzw. Vertragsforschung gegenüber der Lizenznahme gibt es folglich ein zentrales Unterscheidungsmerkmal: Während es sich bei der Auftragsforschung um die Erarbeitung neuen technologischen Wissens handelt, wird bei der Lizenznahme das bereits existierende Wissen erworben. ${ }^{74}$

Die Erscheinungsvielfalt der kooperativen FuE ist zweifelsohne am größten und umfasst ein Spektrum von informellen Erfahrungsaustauschbeziehungen bis zur Gründung eines Gemeinschaftsunternehmens und bereitet deswegen große Abgrenzungsschwierigkeiten sowohl in der Theorie als auch in der Empirie. ${ }^{75}$ Problematisch ist dabei vor allem, dass sich in der einschlägigen Literatur keine einheitliche Definition dieses Phänomens durchgesetzt hat, so dass auch sehr weite Fassungen des Begriffs vorgefunden werden können. Geht man beispielweise davon aus, dass Kooperation als jede Art der Zusammenarbeit von Wirtschaftssubjekten aufgefasst werden kann, die ein abgestimmtes Verhalten der Akteure verlangt ${ }^{76}$, dann können alle Formen des Markttausches in die Definition mit eingeschlossen werden. Diese sehr weite Fassung ist jedoch mit der institutionenökonomischen Theorierichtung nicht kompatibel, die explizit zwischen Marktverträgen und Kooperationen als „hybriden“ Organisationsformen unterscheidet.

Bei der Abgrenzung zwischen Kooperations- und Marktlösung erscheint es daher angebracht, von der Definition der Markttransaktion als eines Tausches von

72 Vgl. Schneider/Zieringer (1991), S. 31.

${ }^{73}$ Vgl. Schneider/Zieringer (1991), S. 33.

74 Vgl. hierzu auch Pisano (1990), S. 163.

${ }^{75}$ Für genauere Erläuterung der charakteristischen Merkmale einzelner Formen kooperativer FuE vgl. Schneider/Zieringer (1991), S. 34-37.

76 Vgl. Chung (1998), S. 27. 
Gütern gegen Geld auszugehen. ${ }^{77}$ Damit stellen sowohl Lizenznahme als auch Vertragsforschung Markttransaktionen dar, da beide Koordinationsformen das Kriterium der finanziellen Vergütung erfüllen. Grundlage der Kooperationen bildet dagegen der gegenseitige Tausch von Erfahrungen und Ergebnissen der FuE-Tätigkeit, so dass diese Koordinationsart einen Geldtransfer nicht involviert.

Zur weiteren Präzisierung des Begriffs der Vertragsforschung kann weiterhin die Problematik der statistischen Erfassung der Forschungs- und Entwicklungstätigkeit erläutert werden. Bei der Erhebung der Daten zu FuEAufwendungen im Wirtschaftssektor ${ }^{78}$ wird in Übereinstimmung mit den Empfehlungen des Frascati-Manuals zwischen den Aufwendungen für interne und externe Forschung und Entwicklung unterschieden. Interne FuE umfasst alle Mittel, die für die im eigenen Unternehmen durchgeführte FuE aufgewandt werden, unabhängig von der Herkunft der Mittel. ${ }^{79}$ Externe FuE umfasst dagegen alle Mittel, die für die an Dritte vergebenen FuE-Aufträge aufgebracht werden. ${ }^{80}$ Damit ermöglicht das auf dem Frascati-Manual basie:ende Berichtssystem eine statistische Erfassung des Phänomens der Auftragsvergabe und bindet es an das Kriterium der erfolgten bzw. versprochenen Zahlung für eine FuE-Leistung.

Auf der Basis der bisherigen Vorüberlegungen kann nachfolgend eine Definition der Vertragsforschung festgelegt werden, die aufgrund ihrer Allgemeingültigkeit sowohl eine theoretische Auseinandersetzung mit dem Phänomen ermöglicht als auch die Grundlage der empirischen Studie bildet.

Vertragsforschung soll nachfolgend definiert werden als

eine Vereinbarung zwischen Auftraggeber und Auf-
tragnehmer, bei der sich der Auftragnehmer (FuE-
Dienstleister) verpflichtet, eine festgelegte Forschungs-

77 Vgl. Chung (1998), S. 27.

78 Im Frascati-Handbuch wird im Allgemeinen zwischen fünf Sektoren unterschieden: Wirtschaftssektor, Staat, private Organisationen ohne Erwerbszweck, Hochschulsektor und Ausland. Der Wirtschaftssektor umfasst laut Definition alle Unternehmen, Organisationen und Institutionen, deren Hauptzweck im Verkauf von Gütern und Dienstleistungen besteht, einschließlich der privaten Organisationen ohne Erwerbszweck, die von ihnen finanziert werden, vgl. zu dieser Abgrenzung: Stifterverband für die Deutsche Wissenschaft (2004), S. $40^{*}$.

79 Vgl. OECD (2002), S. 108.

80 Vgl. OECD (2002), S. 118 sowie die Abgrenzungen des Fragebogens der deutschen FuE-Statistik: Stifterverband für die Deutsche Wissenschaft (2004), Anhang 3, S. 34*. 


\section{und Entwicklungsaufgabe (FuE-Dienstleitung) durch- zuführen und die Verfügungsrechte an dem gewonnen Wis- sen gegen Vergütung auf den Auftraggeber zu übertragen.}

Die gewählte Definition verwendet bewusst eine enge Auslegung des Begriffs der Vertragsforschung, indem sie das konstituierende Kriterium der finanziellen Vergütung einführt. Dies ermöglicht zum einen eine scharfe Abgrenzung der Vertragsforschung von anderen Organisationsformen für FuE-Leistungen, darunter insbesondere der Kooperationsbeziehungen. Zum anderen erlaubt eine solche Definition die empirische Abgrenzung des Phänomens über die in der FuE-Statistik verwendete Kategorie der „externen FuE-Aufwendungen“.

Der Vorteil dieser begrifflichen Festlegung begründet sich ferner in der Tatsache, dass sie sowohl die Perspektive des Auftragnehmers als einer durchführenden Einheit als auch die des Auftraggebers als einer finanzierenden Einheit erfassen läßt. Daher kam auch bei der Benennung des Definiendums der Ausdruck „Vertragsforschung“, und nicht „Auftragsforschung“ zum Einsatz. Letztere Bezeichnung stellt nach der in der Literatur weit verbreiteten Auffassung die Perspektive der Auftragnehmer in den Vordergrund ${ }^{81}$ Zudem erscheint der Begriff der Vertragsforschung vor dem Hintergrund des international etablierten Terminus „contract research“ anschlussfähiger. ${ }^{82}$

\subsection{Eigenschaften des Gutes Wissen}

In Abschnitt 2.1 ist Wissen als wesentlicher Input- und zugleich Outputfaktor im Forschungs- und Entwicklungsprozess herausgestellt worden. In Abschnitt 2.2 wurde zudem deutlich, dass neues, innovationsrelevantes Wissen das eigentliche Transaktionsobjekt bei den FuE-Vertragsbeziehungen darstellt. Ziel der nachfolgenden Ausführungen ist es, das ökonomische Gut Wissen auf seine Eigenschaften hin zu untersuchen.

In der Literatur können zwei grundlegend verschiedene Auffassungen vorgefunden werden. Die Konzeptualisierung von Wissen als einer frei zugänglichen und sich rasch verbreitenden Information wird in Abschnitt 2.3.1 erläutert. Die alternative Auffassung, nach der Wissen eine spezifische, kumulative und implizite Ressource darstellt, wird in Abschnitt 2.3.2 diskutiert.

\footnotetext{
$81 \mathrm{Vgl}$. Corsten (1998), S. 154.

82 Vgl. Rüdiger (2000), S. 27.
} 


\subsubsection{Wissen als Information}

Wissen als Information weist einige nicht unproblematische Eigenschaften auf. Es ist einfach erkennbar bzw. nachschlagbar, frei erhältlich und kann reibungslos aufgenommen, adäquat interpretiert und problemlos umgesetzt werden. $\mathrm{Da}$ der Empfang neuer Informationen ohne Verständnisprobleme erfolgt, sind Ergebnisse industrieller Forschung und Entwicklung der Gefahr ausgesetzt, ohne Zeitverzögerung von Dritten übernommen und reproduziert zu werden. Die Urheber haben kaum Möglichkeiten, die verbreitete Nutzung und unkontrollierte Diffusion zu unterbinden.

Grundlegend für die Auffassung des Wissens als einer frei zugänglichen und rasch diffundierenden Information ist die Verbindung mit dem Konzept der öffentlicher Güter. Wissen besitzt demnach zwei zentrale Eigenschaften der öffentlicher Güter: fehlende Konsumrivalität und Nichtanwendbarkeit des Ausschlussprinzips. ${ }^{83}$

Fehlende Konsumrivalität bedeutet, dass der zusätzliche Gebrauch von Wissen durch einen weiteren Nutzer zu Grenzkosten von Null möglich ist. Im Gegensatz zu materiellen bzw. physischen Gütern kommt es beim "Konsum" von Wissen zu keiner Abnutzung; weder Quantität noch Qualität des vorhandenen Wissensstandes werden durch vermehrte Inanspruchnahme reduziert bzw. beeinträchtigt. ${ }^{84}$ Nichtanwendbarkeit des Ausschlussprinzips bezieht sich darauf, dass das unter Einsatz von knappen Ressourcen produzierte Wissen potenziell allen Wirtschaftsakteuren zur Verfügung steht; Dritte können von der unentgeltlichen Nutzung nicht ausgeschlossen werden. Beispielweise kann jedes neu erarbeitete Konzept zur Senkung der Produktionskosten von Konkurrenten rasch imitiert und angewandt werden; von der unterstellten Korrektur des Preises profitieren alle Konsumenten. In diesem Sinne gehen von der Produktion des Gutes Wissens positive externe Effekte aus. ${ }^{85}$

Beim Vorliegen positiver externer Effekte kommt es jedoch zur Verletzung des Äquivalenzprinzips, das eine Voraussetzung für effiziente Marktallokation bildet. Erhalten die Urheber des Wissens am Markt keine angemessene Vergütung, so ist die Anreizwirkung des Marktes gestört, und es besteht die Ge-

83 Vgl. Kreuzer (1986), S. 89; zur allgemeinen Diskussion der Eigenschaften der öffentlicher Güter vgl. auch Musgrave/Musgrave/Kullmer (1975), S. 55ff., zur lehrbuchartigen Erläuterung vgl. Fritsch/Wein/Ewers (1996), S. 88-90.

84 Vgl. Kreuzer (1986), S. 89.

85 Vgl. Hansen (1999), S. 38. 
fahr, dass die privatwirtschaftlichen Investitionen in Forschung und Entwicklung unter das gesamtwirtschaftlich optimale Niveau fallen. ${ }^{86}$ Aus volkswirtschaftlicher Sicht hat das eine suboptimale Versorgung mit dem Gut Wissen zur Folge und rechtfertigt als Erscheinungsform des Marktversagens staatliche Eingriffe zur Korrektur von Entscheidungen der Marktakteure. ${ }^{87}$ Drei Handlungsalternativen stehen dem Staat in diesem Falle zur Auswahl: eine Subventionslösung (sog. Pigou-Subvention), die Gewährung exklusiver Verfügungsrechte an Erfindungen und eine staatliche Versorgung. ${ }^{88}$

Die Auffassung von Wissen als Information führt nicht nur zur Erkenntnis des Marktversagens im Bereich der Forschung und Entwicklung, sondern hat auch schwerwiegende Folgen für die Frage nach der Marktfähigkeit von Inventionen. Zwecks Ermittlung des Kaufpreises muss der Verkäufer dem Käufer den Inhalt des Gutes erklären. Ist die Information jedoch offengelegt worden, besteht für den Käufer kein Anreiz mehr, in die Transaktion einzutreten. Dieses als Informationsparadoxon in der Literatur diskutierte Problem impliziert, dass in der Abwesenheit tauschfähiger Rechtstitel am Wissen (Patente) Markttransaktionen unmöglich sind und die interne Verwertung des Wissens die einzige plausible Alternative darstellt. ${ }^{89}$

\subsubsection{Wissen als Know-how}

In einer alternativen Konzeptualisierung kann eine Dichotomisierung vorgenommen werden: Der in einer Volkswirtschaft vorliegende Wissensbestand

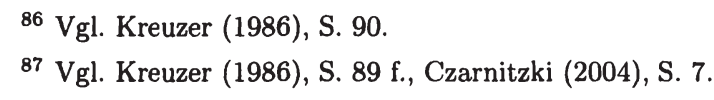

88 Die Pigou-Lösung besteht darin, die potenziellen Wissensproduzenten so zu subventionieren, dass sich die sozialen und privaten Grenzkosten beim gesamtwirtschaftlichen Optimum angleichen, vgl. Fritsch/Wein/Ewers (1996), S. 94. Die Gewährung der exklusiven Verfügungsrechte am Wissen hat zum Ziel, den Erfindern ein zeitlich befristetes Recht der Alleinnutzung einer Invention $\mathrm{zu}$ erteilen, wodurch eine unentgeltliche Inanspruchnahme durch Dritte unterbunden wird, d.h. die Ausschlussmöglichkeit wiederhergestellt wird. Bei der staatlichen Versorgung wird auf die Inanspruchnahme von der Steuerungsfunktion des Marktes ganz verzichtet. Statt dessen übernimmt der Staat die Aufgabe der Bereitstellung des Gutes Wissen, wobei die Kosten aus allgemeinen Haushaltsmitteln bezahlt werden, vgl. Fritsch/Wein/Ewers (1996), S. 88. Ein Beispiel hierfür stellen die öffentlich finanzierten Einrichtungen dar, die mit der Aufgabe der Grundlagenforschung beauftragt sind. Für eine kritische Auseinandersetzung mit der Wirksamkeit dieser Lösungen vgl. u.a. Dubbermann (1999), Fritsch (1993) bzw. Kreuzer (1986).

89 Zur klassischen Betrachtung vgl. Arrow (1962). 
kann in explizites Wissen mit den Attributen einer Information und implizites Wissen aufgeteilt werden. ${ }^{90}$ Explizites Wissen ist kodifiziert und in Form von Blaupausen oder Formeln jedermann zugänglich; implizites Wissen ist dagegen nicht artikuliert bzw. nicht artikulierbar und basiert weitestgehend auf individuellen Erfahrungen und Fertigkeiten der Menschen.

In der Literatur wird für die Beschreibung impliziten Wissens auch die Bezeichnung Know-how verwendet ${ }^{91}$ wobei Know-how in Anlehnung an von Hippel als "the accumulated practical skill or expertise which allows one to do something smoothly and efficiently"92 umschrieben werden kann. Der Rückgriff auf den Begriff „Know-how" verdeutlicht den prozessualen Charakter impliziten Wissens und stellt seine technische Komponente in den Vordergrund. ${ }^{93}$ Implizites Wissen als Know-how konzeptualisiert umfaßt demnach die Gesamtheit der individuellen Fertigkeiten und Erfahrungen eines Menschen, die in einem kontext-spezifischen Umfeld aufgebaut wurden, praktischer Natur sind und zur Lösung verwandter Problemstellungen verwendet werden können. ${ }^{94}$

Für die Abgrenzung von Know-how als einer Komponente des volkswirtschaftlichen Wissensbestandes können folglich zwei Kriterien herangezogen werden: die Art der Wissenserzeugung und die Zugänglichkeit für Dritte. Implizites Wissen wird durch persönliche Erfahrungen im Umgang mit bestimmten Aufgaben und Problemstellungen aufgebaut. Die Übertragung impliziten Wissens ist mit Lernprozessen im engen Kontakt zwischen dem Urherber und dem Adressat verbunden und erfolgt vorwiegend über Beobachtung, Imitation und praktisches Training. ${ }^{95}$

Wissen als Know-how konzeptualisiert weist folglich nur eingeschränkt die Eigenschaften eines öffentlichen Gutes auf. Die Nichtrivalität im Konsum bleibt zwar erhalten; da jedoch die Übermittlung von Know-how die Transferbereitschaft des Urhebers voraussetzt, der gewillt sein muss, seine Fertigkeiten durch Vorführen zum Ausdruck zu bringen, können Dritte von der Nutzung ausgeschlossen werden. Einer raschen Diffusion von implizitem Wissen wirken zudem

90 Vgl. Nonaka (1994), S. 15 f., Takii (2004), S. 81. Der Begriff des impliziten Wissens (tacit knowledge) geht auf die Arbeit von Polanyi (1958) zurück.

$91 \mathrm{Vgl}$. Kogut/Zander (1992), S. 386.

92 von Hippel (1987), S. 291.

93 Zur Unterscheidung zwischen der technischen und der mentalen Dimension impliziten Wissens vgl. Nonaka (1994), S. 16.

94 Für eine ausführliche Analyse der Problematik der definitorischen Abgrenzung des impliziten Wissens vgl. insbesondere Rüdiger/Vanini (1998), S. 468-472.

95 Vgl. Chung (1998), S. $164 \mathrm{f}$. 
die damit verbundenen hohen Kosten entgegen, da Erlernen von Fertigkeiten ein zeitaufwendiger Prozess ist. 


\section{Kapitel 3}

\section{Bestimmungsgründe der FuE-Tätigkeit im Wirtschaftssektor}

Auf dem Gebiet der Innovationsökonomik sind seit den grundlegenden Werken von Schumpeter zahlreiche Hypothesen und Modelle entwickelt worden, die sich zum Ziel gesetzt haben, das Innovationsverhalten der Wirtschaftsakteure auf der Mikroebene theoretisch zu rekonstruieren. Vermutlich ist gerade aufgrund der Vielfalt von formulierten Aussagen bisher kein geordnetes Theoriegebäude entstanden ${ }^{96}$; vielmehr existieren auch in der jüngeren Literatur diverse, zum Teil konkurrierende Hypothesen nebeneinander.

Die in der theoretischen Auseinandersetzung mit dem Phänomen der Wissensproduktion im Wirtschaftssektor aufgestellten Hypothesen wurden jedoch in den letzten Jahrzehnten vielfältiger empirischer Prüfung unterzogen. Dank der empirischen Forschung kann die Stichhaltigkeit der Annahmen einzelner Modelle sowie der im Einzelnen postulierten Zusammenhänge beurteilt werden. Daher sollen im folgenden Überblick die formulierten Hypothesen über die Bestimmungsgründe der FuE-Tätigkeit den Befunden der empirischen Forschung gegenübergestellt werden. Dieses Verfahren wird es ermöglichen, den Stand der Forschung zu den Determinanten der FuE-Tätigkeit nachzuzeichnen, die aus der Fülle der empirischen Studien hervorgehenden robusten Ergebnisse und stilisierten Fakten zu identifizieren und zugleich Erkenntnisse bezüglich der Methodologie der empirischen Innovationsforschung zu gewinnen.

96 Vgl. Frisch (1993), S. 12. 
Zur Auswahl der im Hinblick auf die Schwerpunktlegung der vorliegenden Arbeit relevanten empirischen Evidenz sind folgende Kriterien herangezogen worden:

1. In der nachstehenden Zusammenstellung sind nur diejenigen Studien berücksichtigt, die als Regressanden Indikatoren der auf Unternehmensbzw. Branchenebene erfassten Forschungstätigkeit verwenden. Nur so können Erkenntnisse über die Bestimmungsgründe der FuE-Tätigkeit gewonnen werden. Aus der Analyse ausgeschlossen wurden folglich jene Studien, welche Determinanten des breit verstandenen Innovationsgeschehens untersuchen. ${ }^{97}$

2. Aus zwei Gründen werden schwerpunktmäßig Ergebnisse der neueren, d.h. nach 1990 veröffentlichten Untersuchungen näher beleuchtet: Zum einen liegen bereits Übersichtsstudien vor, die Befunde älterer empirischen Arbeiten, insbesondere derjenigen zu den Neo-SchumpeterHypothesen, systematisieren. ${ }^{98}$ Die Ergebnisse dieser Studien werden stellenweise als Ausgangspunkt für die weitere Analyse verwendet. Zum anderen ist die Aussagekraft der älteren Beiträge aufgrund der Problematik der Datenermittlung zum Teil eingeschränkt. Diese Arbeiten verwenden nicht nur kleinere Datensätze, sondern beschränken sich zudem häufig auf die Gruppe der Großunternehmen bzw. der börsennotierten Unternehmen.

3. Erläutert werden Ergebnisse derjenigen ökonometrischen Studien, in denen für eine Vielzahl von Unternehmens- und Branchencharakteristika kontrolliert wird. Schlussfolgerungen aus den zahlreichen Fallstudien bzw. deskriptiven Datenauswertungen werden nur zur Vertiefung bzw. Exemplifizierung der erläuterten empirischen Befunde herangezogen.

Das Kapitel ist folgendermaßen aufgebaut: Zunächst wird in Abschnitt 3.1 auf die Problematik der Messung von FuE-Tätigkeit eingegangen. Ziel der Ausführungen ist es dabei, die in der empirischen Forschung verwendeten Indikatoren der Forschungstätigkeit - in Abgrenzung zu Indikatoren der Innovationstätigkeit - zu identifizieren. In Abschnitt 3.2 erfolgt die Erläuterung der

97 Zur genauen Abgrenzung der relevanten Indikatoren vgl. Abschnitt 3.1.

98 Vgl. Symeonidis (1996), Cohen (1995), Frisch (1993), Cohen/Levin (1989), Baldwin/Scott (1987), Kamien/Schwartz (1982), Mowery/Rosenberg (1979), Kamien/Schwartz (1975). 
grundlegenden Erkenntnisse von Schumpeter, der als Gründungsvater der Innovationsökonomik gilt und dessen Hypothesen das Untersuchungsdesign der empirischen Innovationsforschung nachhaltig geprägt haben, sowie die Darstellung der Ergebnisse der empirischen Tests der sogenannten Neo-SchumpeterHypothesen. In Abschnitt 3.3 wird auf einzelne unternehmensinterne Determinanten der FuE-Tätigkeit eingegangen. In Abschnitt 3.4 sollen die in der Tradition Schumpeters stehenden Hypothesen über die Bestimmungsgründe der Forschungs- und Entwicklungstätigkeit im Wirtschaftssektor durch die Erkenntnisse der Industrieökonomik ergänzt werden. Eine Zusammenfassung der Ergebnisse in Abschnitt 3.5 schließt die Ausführungen dieses Kapitels ab.

\subsection{Messung der FuE-Tätigkeit}

Empirische Innovationsforschung umfasst sowohl Studien zu den Determinanten der Forschungs- als auch der Innovationstätigkeit, d.h. liefert Hinweise auf die Bestimmungsgründe des breit verstandenen Innovationsgeschehens. Da jedoch in der vorliegenden Arbeit das Phänomen der Wissensproduktion im Wirtschaftssektor - und nicht das weit gefasste Innovationsgeschehen - untersucht wird, ist es notwendig, diejenigen auf dem Gebiet der „empirischen Innovationsforschung " durchgeführten Untersuchungen zu identifizieren, die Erkenntnisse über die eng gefasste Forschungstätigkeit der Unternehmen liefern können. Daher gilt es im Folgenden auf die in den empirischen Untersuchungen verwendeten Indikatoren einzugehen, um so diejenigen Maße zu ermitteln, die als Indikatoren der Forschungstätigkeit aufgefasst werden. Bei der Vorstellung der einzelnen Maße sollen explizit die relativen Vor- und Nachteile der einzelnen Indikatoren aus der Perspektive der empirischen Innovationsforschung erläutert werden.

Das Frascati-Handbuch empfiehlt eine rein inputorientierte Messung der FuETätigkeit anhand zweier Indikatoren: FuE-Aufwendungen und FuE-Personal. ${ }^{\mathbf{9 9}}$ Es wird zwar erkannt, dass mit der Fokussierung auf den FuE-Input der aus volkswirtschaftlicher Sicht relevante Aspekt der Gewinnung neuer Erkenntnisse außer Acht gelassen und der Schwerpunkt stattdessen allein auf den geleisteten Aufwand gelegt wird. ${ }^{100}$ Allerdings werden mit dem Verweis auf die Schwierig-

99 Vgl. OECD (2002), S. 17 und S. 20.

100 OECD (2002), S. 17. Auch in der innovationsökonomischen Literatur wird eine solche einseitige Fokussierung häufig bemängelt, vgl. hierzu Kleinknecht/van Montfort/Brouwer (2000), S. 3, Jacobsson/Oskarsson/Philipson (1996), S. 577, Coombs/Narandren/Richards 
keiten der statistischen Abgrenzung und Erfassung des FuE-Outputs lediglich Richtlinien zur Messung der Inputseite formuliert.

Die Vorteile dieser Messgrößen aus der Perspektive der empirischen Innovationsforschung liegen in der relativen Einfachheit der Erfassbarkeit, der leichten Zugänglichkeit aufgrund der langen Praxis der Erhebung sowie - insbesondere im Falle des Indikators FuE-Personal - guten Eignung für internationale Vergleiche. Nachteilig wirkt sich in erster Linie aus, dass die inputorientierte Messung der FuE-Tätigkeit zur tendenziellen Unterschätzung des Forschungsaufwandes der Klein- und Mittelunternehmen (KMU) führt. Die in der innovationsökonomischen Literatur weit verbreitete These der Unterschätzung der FuE von KMU stützt sich dabei vorwiegend auf die Ergebnisse der Studie von Kleinknecht und Reijnen (1991), in der offizielle Statistiken zur FuE-Tätigkeit der niederländischen Unternehmen mit Daten aus zwei alternativen Quellen verglichen wurden und erhebliche Abweichungen, mitunter eine gewichtige Unterbewertung des Forschungsaufwandes der Klein- und Mittelunternehmen in den offiziellen Statistiken, festgestellt werden konnten. Die in der Subpopulation der Klein- und Mittelunternehmen besonders ausgeprägten Divergenzen ließen sich zum Teil auf eine unterschiedliche Erfassung der statistischen Einheiten (Unternehmen) in den jeweiligen Datenquellen zurückführen. Die wichtigste Ursache für die Unterschiede sahen die Autoren jedoch in der Natur der Forschungstätigkeit der KMU und dem daraus resultierenden Meldeverhalten. Da Klein- und Mittelunternehmen oft diskontinuierlich, informal (d.h. ohne FuE-Abteilung) und in geringem Umfang FuE-Tätigkeit aufnehmen, kann vermutet werden, dass sie eine geringere Neigung aufweisen, Angaben über die eigenen FuE-Aufwendungen an das statistische Zentralamt weiterzuleiten. ${ }^{101}$

Aufgrund der erkannten Defizite der rein inputorientierten Messung werden in der empirischen Innovationsforschung auch output- und marktorientierte Indikatoren, sowie - insbesondere in der neuesten Literatur - Gesamt- bzw. Globalindikatoren (composite indicators) verwendet. Letztere werden meist mit Hilfe der Faktoranalyse aus den Einzelindikatoren gewonnen. ${ }^{102}$ Die meistverwendeten Maße, die den jeweiligen Gruppen zugeordnet werden können, sind

(1996), S. 404.

$101 \mathrm{Vgl}$. Kleinknecht/Reijnen (1991) sowie Kleinknecht (1987). In der ersten Studie werden zugleich die Konsequenzen der Unterschätzung des Forschungsaufwandes der KMU für Ergebnisse der empirischen Innovationsforschung erläutert und exemplifiziert. Für die Bestätigung der Hypothese der Unterschätzung des Forschungsaufwandes der KMU in den offiziellen Statistiken anhand von Daten zur Innovationstätigkeit italienischer Unternehmen vgl. Santarelli/Sterlacchini (1990).

${ }^{102}$ Auf die Herleitung der Gesamtindikatoren wird nicht näher eingegangen, da diese meist 
in Tab. 3.1 zusammengestellt worden. Wie ihr entnommen werden kann, finden neben metrisch skalierten Variablen (FuE-Aufwand, Innovationsaufwand, Umsatzanteile) auch ordinal skalierte Variablen (Neuheitsgrad, Beurteilung aus technologischer bzw. ökonomischer Sicht) Verwendung. Während die Ersteren auf die quantitative Messung des Innovationsaufwandes bzw. -erfolges abstellen, wird mit der Verwendung der ordinalskalierten Variablen das Ziel verfolgt, mehr auf die „Qualität" der Innovationstätigkeit abzustellen.

Für den weiteren Verlauf der vorliegenden Forschung sind im Einklang mit der in Abschnitt 2.1.3 vorgenommenen Abgrenzung nur diejenigen Indikatoren von Interesse, die als Messgrößen der Forschungstätigkeit aufgefasst werden können und nicht das breit verstandene Innovationsphänomen abbilden sollen. Ein Vergleich der Definition der FuE-Tätigkeit im Frascati-Manual mit den Definitionen, die bei der Erhebung der Daten verwendet werden und die Grundlage für die in der Tabelle 3.1 aufgeführten Indikatoren bilden ${ }^{103}$, führt zu dem Ergebnis, dass die Patenttätigkeit als ein Indikator der Outputseite der FuE-Tätigkeit verwendet werden kann. Allerdings kann dies nur bedingt erfolgen, was nachfolgend begründet wird.

Patente als Indikator für die Anzahl der durch eine Unternehmung hervorgebrachten Inventionen fanden insbesondere in der älteren Literatur starke Verwendung aufgrund der leichten Zugänglichkeit der entsprechenden Daten, der direkten Verknüpfung mit dem Innovationsgeschehen und der postulierten Objektivität dieser Messgröße. ${ }^{104}$ Allerdings ist die Verwendung der Patentzahlen als einer quantitativen Messgröße i.d.R. mit mehreren Problemen behaftet. Zum einen bestehen erhebliche qualitative Unterschiede zwischen den rein quantitativ betrachteten Schutzrechten: Patente können sowohl für ganze Systeme von verbundenen Inventionen erteilt werden als auch für einzelne Kom-

auf eine möglichst breite Abbildung des Innovationsgeschehens abzielen. Vgl. dazu Arvanitis/Hollenstein (1994b), Hollenstein (1996), Hagedoorn/Cloodt (2003).

103 Bei der Erhebung der Daten zu Innovationsaufwendungen wird meist die Definition des Oslo-Manuals zugrundegelegt. Laut der Definition dieses Handbuchs zählen zu den Innovationsaufwendungen neben den FuE-Aufwendungen: (1) Ausgaben für externes Wissen (disembodied technology and know-how) wie beispielweise Patente und Lizenzen, (2) Ausgaben für Maschinen und Sachmittel (embodied technology), (3) Ausgaben für Konstruktion, Produktgestaltung, Prototypen und Vorbereitungen für die Produktion, (4) Weiterbildungsausgaben und (5) Ausgaben für die Markteinführung von Produkten, vgl. OECD/Eurostat (1997), S. 87.

$104 \mathrm{Vgl}$. Griliches (1990), S. 1661. Für eine kompakte, tabellarische Zusammenstellung der Vor- und Nachteile der Patentzahlen aus der Sicht der empirischen Innovationsforschung vgl. insbesondere Archibugi/Pianta (1996), S. 22. 
Tabelle 3.1: Indikatoren der Innovationstätigkeit

\begin{tabular}{|c|c|}
\hline Beschreibung & Skalenniveau \\
\hline \multicolumn{2}{|l|}{ Inputorientierte Indikatoren } \\
\hline FuE-Tätigkeit (ja/nein) & nominal \\
\hline FuE-Aufwendungen & metrisch \\
\hline FuE-Aufwendungen als Umsatzanteil & metrisch \\
\hline FuE-Personal & metrisch \\
\hline Anteil FuE-Personal an der Gesamtzahl der Beschäftigten & metrisch \\
\hline Anteil der Beschäftigten mit höherer technischer Qualifikation & metrisch \\
\hline Innovationstätigkeit (ja/nein) & nominal \\
\hline Innovationsaufwendungen & metrisch \\
\hline Innovationsaufwendungen als Umsatzanteil & metrisch \\
\hline \multicolumn{2}{|l|}{ Outputorientierte Indikatoren } \\
\hline Patentaktivität (ja/nein) & nominal \\
\hline Anzahl Patente & metrisch \\
\hline Forschungsproduktivität (Anzahl Patente/FuE-Aufwendungen) & metrisch \\
\hline Anzahl Innovationen & metrisch \\
\hline Neuheitsgrad der Innovationen & ordinal \\
\hline Beurteilung der Innovationen aus technischer Sicht & ordinal \\
\hline Beurteilung der Innovationen aus ökonomischer Sicht & ordinal \\
\hline Erzielte Kostenreduktionen & metrisch \\
\hline \multicolumn{2}{|l|}{ Marktergebnisorientierte Indikatoren } \\
\hline Umsatzanteil verbesserte Produkte & metrisch \\
\hline Umsatzanteil Marktneuheiten & metrisch \\
\hline
\end{tabular}

Quelle: Eigene Darstellung. 
ponenten. ${ }^{105}$ Zum anderen sind nicht alle Inventionen patentfähig bzw. werden nicht patentiert, da Patente häufig nicht als wirksames Instrument zum Schutz des geistigen Eigentums angesehen werden. ${ }^{106}$ Oft verzichten Unternehmen auf die direkte Veräußerung des erarbeiten Wissens, etwa durch Patenterwerb und Lizenzverkauf, und setzen Inventionen unternehmensintern in neue Produkte bzw. Verfahren um; Forschungsergebnisse werden somit nicht direkt in immaterieller Form, sondern nur indirekt - als sogenanntes „embodied knowlegde“ vermarktet. Folglich unterschätzt diese Messgröße unter Umständen die faktische Forschungstätigkeit in einer Volkswirtschaft. Die besondere Schwäche derjenigen empirischen Untersuchungen, die Patente als outputorientierten Indikator der Forschungstätigkeit verwenden, liegt jedoch in der Annahme einer konstanten Patentneigung unabhängig von Betriebsgrößenklassen, unternehmerischen Strategien, Technologiefeldern und rechtlichen Rahmenbedingungen in den jeweiligen Ländern. Diese Annahme lässt sich jedoch im Lichte der neueren empirischen Forschung zum Patentverhalten der Unternehmen nicht rechtfertigen. Die empirischen Studien zeigen, dass die Patentneigung zwischen den einzelnen Branchen stark variiert, mit der Unternehmensgröße steigt, und von strategischen Abwägungen der Unternehmen geprägt ist. ${ }^{107}$

Im Ergebnis ist bei der Analyse derjenigen Studien, die Patentzahlen als Regressanden verwenden, stets Vorsicht geboten, da in den Untersuchungen meist für die Patentneigung der Unternehmen nicht kontrolliert wird. Folglich stellt sich die Frage, ob die Ergebnisse dieser Studien als Hinweise auf Bestimmungsgründe der FuE-Tätigkeit der Unternehmen verstanden werden sollen oder eher Aussagen über das Patentverhalten der Unternehmen erlauben. Aufgrund der methodologischen Vorbehalte werden in der nachfolgenden Analyse ausgewählte Studien, die Patente als Messgrößen verwenden, nur hilfsweise - zur Abgleichung der Ergebnisse - herangezogen. Ein vollständiger Verzicht auf die Berücksichtigung dieser Untersuchungen erscheint nicht zweckmäßig, weil einige Hypothesen über das Innovationsverhalten der Unternehmen aus der Auseinandersetzung mit diesem Indikator hervorgegangen sind und erst später mit inputorientierten Messgrößen überprüft werden konnten: An dieser Stelle sei exemplarisch die demand-pull-Hypothese genannt, die in Abschnitt 3.4.1 näher erläutert wird.

\footnotetext{
105 Vgl. Duguet/Kabla (2000), S. 269.

106 Vgl. hierzu Abschnitt 3.4.2, S. 80.

107 Vgl. dazu u.a. Duguet/Kabla (2000), Arundel/Kabla (1998), Kortum/Lerner (1999), Brouwer/ Kleinknecht (1999a).
} 


\subsection{Schumpetersche Erkenntnisse und Neo- Schumpeter-Hypothesen}

Schumpeter gilt als Gründungsvater der Innovationsforschung, da er mit seiner Behauptung, der technische Fortschritt - und nicht die Faktorakkumulation - sei die treibende Kraft der wirtschaftlichen Entwicklung, die Bedeutung der Neuerungsprozesse ausdrücklich betont hat. ${ }^{108}$ Träger der wirtschaftlichen Neuerungen ist in dem früheren Werk von Schumpeter, der "Theorie der wirtschaftlichen Entwicklung“, ein Pionierunternehmer, der die künftige Marktentwicklung antizipierend die erfolgversprechendsten Innovationen identifiziert und durchsetzt. ${ }^{109}$ Der dynamische Unternehmer, wie er in dem frühen Werk von Schumpeter dargestellt wird, ist nicht notwendigerweise der Erfinder selbst ${ }^{110}$; er besitzt lediglich vollständige Information über die (exogen) vorhandenen Inventionen und versteht es, die erkannten Marktchancen im Bereich der "neuen Kombinationen“ auszunutzen. Als gestaltungs- und risikofreudiger Gründer stellt er eine Herausforderung für etablierte Unternehmen und Branchen dar; die von ihm durchgesetzten Innovationen verdrängen Produkte der am Markt agierenden Unternehmen. ${ }^{111}$

In seinem späteren Werk "Kapitalismus, Sozialismus, Demokratie“ stellt Schumpeter dagegen große, in konzentrierten Märkten agierende Unternehmen als die wichtigste Quelle der Innovationen heraus:

„Sobald wir auf die Einzelheiten eingehen und die einzelnen Posten untersuchen, bei welchen der Fortschritt am deutlichsten gewesen ist, führt uns die Spur nicht zu den Toren jener Firmen, die unter den Bedingungen einer verhältnismäßig freien Konkurrenz arbeiten, sondern ausgerechnet zu den Toren der großen Konzerne [...]."112

108 „[Die wirtschaftliche Entwicklung ist] nicht einer quasi-automatischen Bevölkerungsund Kapitalzunahme oder den Launen des Geldsystems zuzuschreiben [...]. Der fundamentale Antrieb, der die kapitalistische Maschine in Bewegung setzt und hält, kommt von den neuen Konsumgütern, den neuen Märkten, den neuen Formen der industriellen Organisation, welche die kapitalistische Unternehmung schafft.", Schumpeter (1942/1987), S. 137.

109 Für die Erläuterung der Funktion des Unternehmers in der Durchsetzung von neuen Kombinationen vgl. Schumpeter (1911/1993), S. $110 \mathrm{ff}$.

110 Zur Unterscheidung der Funktion des „Erfinders“ und der des „Unternehmers“, vgl. Schumpeter (1911/1993), S. 129.

111 Vgl. Meschede (2003), S. 8 f.

112 Schumpeter (1942/1987), S. 135. 
„Wir müssen [...] anerkennen, dass die Großunternehmung zum kräftigsten Motor dieses Fortschritts [...] geworden ist [...]."113

Während Schumpeter in seinem frühen Werk die Rolle des dynamischen Unternehmers in der Durchsetzung von Innovationen am Markt betont, widmet er sich in seinem späteren Werk auch der Frage nach der Entstehung der Invention. Eine Erfindung wird nicht mehr als ,exogene Variable des Wirtschaftsprozesses"114 aufgefasst; sie steht nicht als freies Gut zur Verfügung, sondern wird in einem planmäßigen, arbeitsteiligen Prozess - vorwiegend in einer Großunternehmung - erzeugt. Die Großunternehmung wird somit zur Quelle der Inventionen und zum Motor des wirtschaftlichen Fortschritts zugleich.

Die Gründe für die von Schumpeter postulierte technologische Überlegenheit der Großunternehmen sind von zahlreichen in der Tradition Schumpeters argumentierenden Autoren diskutiert worden und lassen sich folgendermaßen zusammenfassen: ${ }^{115}$

- Da die Forschungstätigkeit mit erheblichem finanziellen Aufwand verbunden ist, begründet sich der wichtigste Vorteil größerer, in konzentrierten Märkten agierenden Unternehmen in ihrer Fähigkeit, höhere interne Ressourcen zur Finanzierung der Forschungsvorhaben aufzubringen.

- An der Eigenschaft der hohen internen Unsicherheit setzt das zweite Argument für die technologische Überlegenheit größerer Wirtschaftseinheiten an. Großunternehmen genießen demnach Vorteile der breiten Risikostreuung, da sie „Portefeuille an Forschungsprojekten" ${ }^{\text {"116 }}$ unterhalten können und somit nicht von dem kommerziellen Erfolg von Einzelprojekten in dem Maße abhängen, wie es im Falle der Klein- und Mittelunternehmen die Regel ist.

- Ein weiterer Vorteil größerer Wirtschaftseinheiten gründet sich auf deren Möglichkeit, koordinierte Arbeitsteilung in formalisierten FuEAbteilungen einzuführen und von der daraus resultierenden Spezialisierung zu profitieren.

113 Schumpeter (1942/1987), S. 174 f.

114 Keßler (1992), S. 26.

115 Vgl. Cohen (1995), S. 184 f., Schmidt/ElBer (1990), S. 557, Meschede (2003), S. 12 ff., Arrow (1962), S. 616, Frisch (1993), S. 85, Keßler (1992), S. 35 f., Acs/Audretsch (1990), S. $39 \mathrm{f}$.

116 Schmidt/Elßer (1990), S. 557. 
- Komparative Vorteile der Großunternehmen entstehen ferner aufgrund der technischen Gegebenheiten, die einen mindestoptimalen FuEAufwand erfordern. Beispielsweise kann eine Auslastung von großen Forschungsanlagen eher in Großunternehmen gewährleistet werden. Die Problematik der Unteilbarkeiten wird auch herangezogen, um die These von den wachsenden Skalenerträgen im FuE-Bereich zu begründen, die den komparativen Vorteil größerer Wirtschafteinheiten bekräftigen würde.

- Setzt man voraus, dass das erarbeitete Wissen nicht in immaterieller Form veräußert, sondern unternehmensintern im Produktionsbereich verwertet wird, so begründet sich ein weiterer Vorteil der Großunternehmen in ihrer Fähigkeit, die Kosten der Forschungstätigkeit auf eine größere Absatzmenge zu verteilen (sog. cost-spreading advantage ${ }^{117}$ ).

- Schließlich sind Großunternehmen in der Regel stärker als Kleinunternehmen im Produktionsbereich diversifiziert. Hierdurch entstehen größenbedingte Vorteile, da für die Ergebnisse eigener Forschungstätigkeit ein breiteres unternehmensinternes Anwendungsfeld gegeben ist. Zudem wird auch davon ausgegangen, dass unerwartete Ergebnisse der FuE-Tätigkeit ("Zufallsentdeckungen") eher in großen, diversifizierten Unternehmen intern verwertet werden können, wodurch der erwartete Ertrag der Investitionen in Forschung und Entwicklung in größeren Wirtschaftseinheiten höher ausfällt.

Kleine Unternehmen genießen dagegen Vorteile, die im Organisationsbereich angesiedelt bzw. behavioraler Natur sind. ${ }^{118}$ Es wird davon ausgegangen, dass Innovationsentscheidungen in KMUs von heterogenen Mitarbeitergruppen im unbürokratischen Umfeld getroffen werden, was die Wahrscheinlichkeit der korrekten Identifizierung aussichtsreicher Inventionen erhöht. ${ }^{119}$ Kleinunternehmen wird die Fähigkeit zu schnelleren Anpassungsreaktionen, Risikofreude sowie eine leistungsfördernde, direkte Verbundenheit der Mitarbeiter mit den Forschungsprojekten und Unternehmenszielen zugestanden. Durch hierarchische Strukturen gekennzeichnete Großunternehmen reagieren dagegen verspätet auf die dem Wandel unterliegenden Marktanforderungen und Kundenbedürfnisse; Professionalisierung des Managements führt zur Entfremdung von

117 Vgl. dazu Cohen/Klepper (1996a), S. 926, Cohen/Klepper (1996b), S. 232.

118 Vgl. Rothwell/Dodgson (1991), S. 128.

119 Vgl. Meschede (2003), S. 14. 
technischen Produktionsbedingungen. Die Durchsetzung neuer Forschungsprojekte durch die FuE-Mitarbeiter im Rahmen einer Großunternehmung erweist sich folglich als schwieriger als eine technologieintensive Ausgründung. ${ }^{120}$

Die auf Schumpeter zurückgehenden Überlegungen zur technologischen Überlegenheit größerer Wirtschaftseinheiten sind in der späteren Literatur auf zwei zentrale Thesen verdichtet worden, die als Neo-Schumpeter-Hypothesen (NSH) Grundlage zahlreicher empirischer Arbeiten zur Problematik der Forschungstätigkeit im Wirtschaftssektor geworden sind. Die erste These (NSH 1) stellt den positiven Zusammenhang zwischen der Unternehmensgröße und Forschungstätigkeit heraus. Da Schumpeter die technologische Überlegenheit von Großunternehmen letztlich aus deren spezieller Konkurrenzsituation ableitete $^{121}$, postuliert die zweite Hypothese (NSH 2) einen positiven Zusammenhang zwischen Marktkonzentration und Forschungstätigkeit.

Die empirische Evidenz zu diesen Hypothesen soll in den nächsten Abschnitten näher betrachtet werden.

\subsubsection{Unternehmensgröße und Forschungstätigkeit}

Obwohl die einzelnen Gründe für die in der ersten Neo-Schumpeter-Hypothese postulierte technologische Überlegenheit der Großunternehmen früh erkannt wurden, verfolgten viele empirischen Studien einen vereinfachten Untersuchungsansatz, indem sie den Einfluss von verschiedenen Maßen der Unternehmensgröße (wie z.B. Umsatz, Beschäftigtenzahl oder Bilanzvermögen) auf die Forschungsintensität direkt untersuchten - und nicht die empirische Stichhaltigkeit der im einzelnen genannten Argumente überprüften. ${ }^{122}$ Es zeigte sich

\footnotetext{
120 Vgl. Meschede (2003), S. $14 \mathrm{f}$.

${ }^{121}$ Zur unterlassenen Trennung von Unternehmensgröße und Marktform bei Schumpeter vgl. insbesondere Keßler (1992), S. 36 f.

122 Obwohl die Untersuchung der Stichhaltigkeit einzelner Argumente für die postulierte technologische Überlegenheit der Großunternehmen der von Schumpeter empfohlenen methodologischen Vorgehensweise folgen würde. Für Schumpeter war nicht die endgültige soziale Prognose das Bewertungsmaß der wirtschaftswissenschaftlichen Theorie; vielmehr ging es um die Identifikation der Bestimmungsgründe ausgewählter Phänomene, die eine bestimmte Entwicklung zur Folge haben können, vgl. dazu die Aussage Schumpeters: „Was bei jedem Versuch einer sozialen Prognose zählt, ist nicht das Ja oder Nein, das die dazu führenden Tatsachen und Argumente zusammenfasst, sondern diese Tatsachen und Argumente selbst. Diese enthalten alles, was am endgültigen Ergebnis wissenschaftlich ist. Alles andere ist nicht Wissenschaft, sondern Prophezeiung.", Schumpeter (1942/1987), S. 105.
} 
jedoch, dass dieser Untersuchungsansatz zu widersprüchlichen Befunden führte; in Abhängigkeit von den Einzelheiten der Modellspezifikation erwies sich der Einfluss als mal positiv und mal negativ. ${ }^{123}$

Cohen und Klepper gelang es jedoch, nach einer umfassenden Analyse der frühen empirischen Evidenz zu der ersten Neo-Schumpeter-Hypothese folgende robusten Ergebnisse zu identifizieren: ${ }^{124}$ Erstens sahen die Autoren die Hypothese bestätigt, dass die Wahrscheinlichkeit, dass ein Unternehmen FuETätigkeit aufnimmt, mit der Unternehmensgröße steigt. Zweitens fanden sie in der Fülle der empirischen Studien keine Bestätigung für das Postulat des überproportionalen Anstiegs des Forschungsaufwandes in der Population der Großunternehmen. Drittens stellten sie fest, dass die empirische Evidenz Hinweise auf die mit der Unternehmensgröße sinkende Forschungsproduktivität - als Verhältniszahl zwischen dem Forschungsaufwand und der Anzahl der erworbenen Patente bzw. hervorgebrachten Innovationen - liefert. Insgesamt schlussfolgerten die Autoren, dass die empirische Evidenz die These der technologischen Überlegenheit der Großunternehmen (NSH 1) nicht bestätigt. ${ }^{125}$ Die einzelnen aus der Auswertung der älteren empirischen Studien hergeleiteten Befunde sollen nun den Ergebnissen neuerer empirischer Untersuchungen gegenübergestellt werden.

Das erste Ergebnis der Analyse von Cohen und Klepper wird durch die Ergebnisse der neueren empirischen Studien bestätigt. Bereits in den deskriptiven Auswertungen, die auf umfangreichen Datensätzen zum Innovationsverhalten europäischer Unternehmen basieren, zeigt sich, dass Klein- und Mittelunternehmen tendenziell seltener als Großunternehmen forschen. ${ }^{126}$ Auch ökonometrische Untersuchungen, die inputorientierte Indikatoren der Forschungstätigkeit verwenden, für mehrere Unternehmens- und Branchencharakteristika kon-

${ }^{123}$ Für eine kompakte, tabellarische Zusammenstellung der älteren Studien vgl. Frisch (1993), S. $25 \mathrm{ff}$.

124 Vgl. Cohen (1995), S. 184-191, Cohen/Klepper (1996a), S. 927-930. Zu gleichen Schlussfolgerungen gelangt auch Symeonidis (1996), S. $10 \mathrm{f}$.

125 Vgl. Cohen/Klepper (1996a), S. 925.

${ }^{126}$ Laut den niederländischen Statistiken aus dem Jahre 1988 lagen die Anteile der forschenden Unternehmen im Verarbeitenden Gewerbe bei 34.6\% in der Gruppe der Unternehmen mit 20 bis 49 Mitarbeitern und bei $89.4 \%$ in der Gruppe der Unternehmen mit mehr als 500 Mitarbeitern, vgl. Brouwer/Kleinknecht (1996), S. 102. Nach den deutschen Daten aus dem Jahre 1992 waren $20 \%$ der Industrieunternehmen mit 4 bis 50 Mitarbeitern in FuE angagiert; das gleiche galt jedoch für etwa $80 \%$ der Unternehmen mit über 1000 Beschäftigten, vgl. Licht/Zoz (2000), S. 310 f. 
trollieren und auf Daten aus verschiedenen europäischen ${ }^{127}$ - und außereuropäischen ${ }^{128}$ - Ländern basieren, bestätigen, dass die Wahrscheinlichkeit der Aufnahme der FuE-Tätigkeit mit der Betriebsgröße signifikant steigt.

Neuere Studien bekräftigen ebenfalls die zweite Schlussfolgerung von Cohen und Klepper, wonach die These des mit der Unternehmensgröße überproportional ansteigenden Forschungsaufwandes empirisch nicht bestätigt werden kann. Crepon et al. (1996), die die Schätzungen anhand der Daten für französische Unternehmen durchführen, können die Hypothese des mit der Unternehmensgröße proportional steigenden Forschungsaufwandes - sowie des proportionalen Anstiegs der Anzahl der gemeldeten Patente - nicht ablehnen. ${ }^{129}$ In der Studie von Lenz (1998, Schweizer Unternehmensdaten) liegt die Umsatzelastizität der FuE-Ausgaben bei einem Wert um 0.7, auf unterproportionale Steigerung der FuE-Aufwendungen hinweisend. ${ }^{130}$ In der auf niederländischen Daten basierenden Untersuchung von Ophem et al. (2002) liegt die Elastizität der FuEAusgaben ebenfalls unter 1 , wobei als Maß der Unternehmensgröße die Mitarbeiterzahl verwendet wird. ${ }^{131}$ Auch Veugelers (1997) kommt in ihrer anhand der Daten zur Forschungstätigkeit belgischer Unternehmen durchgeführten Untersuchung zu dem Ergebnis, dass die FuE-Aufwendungen unterproportional mit der Unternehmensgröße steigen. ${ }^{132}$

In den neueren Studien wird auch die Beziehung zwischen der Forschungsintensität (d.h. dem Anteil der FuE-Aufwendungen am Umsatz bzw. dem Anteil des FuE-Personals an der Gesamtzahl der Beschäftigten) und der Unternehmensgröße untersucht. Hinweise auf einen nichtlinearen, U-förmigen Zusammenhang liefern die Untersuchungen, die auf deutschen Unternehmensdaten basieren ${ }^{133}$ : Demnach nimmt die FuE-Intensität zunächst mit der Unternehmensgröße ab, um dann bei sehr großen Unternehmen wieder anzusteigen.

127 Vgl. Kleinknecht/Oostendorp (2002), S. 313, Leiponen (2002), S. 271, Felder/Licht/Nerlinger/Stahl (1996), S. 141 ff., Crepon/Duguet/Kabla (1996), S. 77, Evangelista/Perani/Rapiti/Archiburgi (1997), S. 526, Arvanitis/von Arx (2004), Tabelle 5.4 und 5.5, Becker/Peters (2005), S. 141.

$128 \mathrm{Vgl}$. für brasilianische Unternehmensdaten: Braga/Willmore (1991), S. 428, und für indische Unternehmensdaten: Kumar/Saqib (1996), S. 718, Siddharthan/Agarwal (1992), S. 106.

129 Vgl. Crepon/Duguet/Kabla (1996), S. 84.

130 Vgl. Lenz (1998), Tabelle 7.8, S. 139.

$131 \mathrm{Vgl}$. van Ophem/Brouwer/Kleinknecht/Mohnen (2002), S. 62 ff. sowie auch Ergebnisse von Vossen/Nooteboom (1996), Tabelle 6.2, S. 162.

132 Vgl. Veugelers (1997), S. 310.

${ }^{133} \mathrm{Vgl}$. hierzu insbesondere die deskriptive Statistik und die Ergebnisse der ökonometri- 
Sehr forschungsintensive Unternehmen finden sich demnach verstärkt sowohl in der Gruppe der kleinen als auch in der Gruppe der großen Unternehmen. Allerdings können diese Ergebnisse nicht als konsensfähig gelten. Studien, die auf Angaben zur Forschungsintensität der Unternehmen aus anderen Ländern basieren, kommen zu sehr unterschiedlichen Ergebnissen, so dass ein allgemeingültiges Muster nicht erkennbar ist. ${ }^{134}$

Für die dritte These von Cohen und Klepper, nach der die FuE-Produktivität mit der Unternehmensgröße sinkt, können in der neueren Literatur ebenfalls einige Belege gefunden werden. Arvanitis (1997) untersucht die Beziehung zwischen dem FuE-Aufwand und der Zahl der gemeldeten Patente für eine Gruppe von 397 Schweizer Unternehmen und stellt dabei fest, dass die Forschungsproduktivität mit der Unternehmensgröße sinkt. ${ }^{135}$ Eine Untersuchung von Licht und $\mathrm{Zoz}$ (2000) anhand von deutschen Unternehmensdaten kommt zu dem Ergebnis, dass die Skalenintensität in FuE für die Mehrheit der Unternehmen den Wert von 1 nicht übersteigt; nur für die Gruppe der größten Unternehmen liefern die deutschen Unternehmensdaten einen Hinweis auf steigende Skalenerträge. ${ }^{136}$

Bei der Interpretation der Ergebnisse zu unterschiedlichen Forschungsproduktivitäten der Unternehmen verschiedener Betriebsgrößenklassen gilt es jedoch die Problematik der input- und outputorientierten Messung von FuE-Aktivität zu beachten, da messtechnische Unterschiede zu einer Verzerrung der ermittelten Zusammenhänge führen können. In diesen Studien wird letztlich die Skalenintensität der FuE-Produktionsfunktion untersucht; die FuE-Aufwendungen fließen als Input in den Produktionsprozess ein und führen zum Output in der

schen Untersuchung für die westdeutschen Unternehmen in Felder/Licht/Nerlinger/Stahl (1996), sowie die Ergebnisse von Becker/Peters (2005), S. 141.

${ }^{134}$ So bestätigt sich in einer Untersuchung von van Dijk et al. (1997), in der Forschungsintensität durch den Anteil des FuE-Personals an der Gesamtzahl der Beschäftigten abgebildet wird, und die Regressionen getrennt nach Betriebsgrößenklassen anhand von niederländischen Daten durchgeführt werden, der negative Einfluss der steigenden Unternehmensgröße auf die Forschungsintensität in der Gruppe der kleinen Unternehmen (unter 100 Vollzeitbeschäftigten). Der Einfluss der Unternehmensgröße in der Gruppe der großen Unternehmen erweist sich jedoch als nicht signifikant, vgl. van Dijk/den Hertog/Menkveld/Thurik (1997), S. 340. Arvanitis formuliert in seiner auf Schweizer Daten basierenden Untersuchung das Ergebnis: „R\&D intensity, i.e. R\&D expenditure per employee, increases with firm size with a decreasing rate up to a certain point", Arvanitis (1997), S. 483.

135 Vgl. Arvanitis (1997), S. 484.

136 Die Autoren verweisen in ihrer Studie auf eine Untersuchung von Crepon und Duguet (1996), deren Ergebnisse auf konstante Skalenerträge schließen lassen, vgl. Licht/Zoz (2000), S. 324 . 
Form von Patenten. Problematisch jedoch ist dabei erstens - wie in Abschnitt 3.1 bereits erläutert - dass die offiziellen Statistiken den FuE-Aufwand der kleinen und mittleren Unternehmen unterschätzen. Der Befund der höheren Forschungsproduktivität der kleineren Unternehmen kann folglich als Ergebnis der Unterbewertung der Inputseite in den FuE-Produktionsfunktionen der KMU zustande kommen. Zweitens, wie ebenfalls in Abschnitt 3.1 angedeutet, unterschätzen Patentzahlen - als outputorientierter Indikator der FuE-Tätigkeit - die effektive Ausbringungsmenge der Forschungsanstrengungen, sind also folglich nur bedingt als Indikator der Outputseite geeignet. In der Literatur können weitere methodologische Vorbehalte identifiziert werden:

- Es ist davon auszugehen, dass das Patentverhalten der Unternehmen im Falle der Prozess- und Produktinnovationen unterschiedlich ausfällt. Da nach Einschätzung der Unternehmen neue Produkte durch Patente effektiver geschützt werden können als neue Verfahren - bei denen $\mathrm{Ge}$ heimhaltung als der effektivste Schutzmechanismus angesehen wird ${ }^{137}$, ist von einer geringeren Patentneigung der Unternehmen auszugehen, die ihre Forschungstätigkeit auf kostensenkende Verfahrensinnovationen ausrichten.

- Gleichzeitig zeigt sich, dass der Anteil der Prozessinnovationen mit der Unternehmensgröße steigt. ${ }^{138}$ Folglich ist davon auszugehen, dass die Patentzahlen als outputorientierter Indikator der FuE-Tätigkeit den Wissensoutput der verstärkt im Bereich der Prozesstechnologien forschenden Großunternehmen tendenziell unterschätzen.

- Studien zum Patentverhalten der Unternehmen liefern ferner einen Hinweis darauf, dass kleinere Unternehmen die Effektivität der rechtlichen Schutzmaßnahmen tendenziell niedriger als Großunternehmen einschätzen und eine geringere Patentneigung aufweisen. ${ }^{139}$ Es kann folglich davon ausgegangen werden, dass im KMU-Sektor nicht nur die Inputsondern auch die Outputseite des FuE-Prozesses unterschätzt wird.

Obwohl die empirische Forschung auf eine niedrigere Forschungsproduktivität der Großunternehmen schließen lässt, führen methodologische Vorbehalte im

137 Vgl. dazu Abschnitt 3.4.2.

138 Vgl. Cohen/Klepper (1996b).

139 Vgl. dazu die Ausführungen in Abschnitt 3.4.2. 
Allgemeinen sowie Überlegungen zur relativen Vorteilhaftigkeit der Fokussierung auf Prozess- bzw. Produktinnovationen in Unternehmen verschiedener Betriebsgrößenklassen im Besonderen zur Schlussfolgerung, dass eher von unterschiedlichen Mustern der Spezialisierung der kleinen und großen Unternehmen auszugehen ist. Großunternehmen forschen demnach verstärkt im Bereich der kostensenkenden Verfahrenstechnologien, da sie die erarbeiteten Prozessinnovationen unternehmensintern breit anwenden und Wettbewerbsvorteile aufgrund des Kostenvorsprungs erzielen können. Kleine Unternehmen zielen in ihrer Forschungstätigkeit dagegen verstärkt auf das Hervorbringen von Produktinnovationen $a b$, weil diese es ermöglichen, den Umsatz zu stabilisieren bzw. auszuweiten und das Unternehmenswachstum herbeizuführen. ${ }^{140}$

Geht man von unterschiedlichen Mustern der Spezialisierung von Unternehmen verschiedener Betriebsgrößenklassen aus, dann kann das methodologische Vorgehen, in dem der Zusammenhang zwischen der Forschungstätigkeit und Unternehmensgröße direkt untersucht wird, kritisch beurteilt werden. Maße der Unternehmensgröße werden in diesen Studien letztlich als Näherungsvariablen (proxy variables) verwendet, die eigentliche, vermutlich mit der Unternehmensgröße einhergehende Vorteile im Technologieprozess abbilden sollen. ${ }^{141}$ Der angemessenere Ansatz zur Prüfung der ersten Neo-SchumpeterHypothese bestünde jedoch darin, nicht allein den Einfluss der Unternehmensgröße zu untersuchen, sondern für die einzelnen bereits in der Literatur erkannten Ursachen der potenziellen technologischen Überlegenheit der Großunternehmen zu kontrollieren. Diesen Ansatz versucht man zu verfolgen, indem man den Einfluss der betriebsspezifischen Eigenschaften, Strategien und Spezialisierungsmuster auf die einzelwirtschaftlichen Entscheidungen im FuE-Bereich untersucht. Die stilisierten Fakten der empirischen Innovationsforschung, die aus der Auseinandersetzung mit dem Einfluss einzelner Unternehmenscharakteristika und -strategien hervorgegangen sind, werden in Abschnitt 3.3 näher betrachtet.

Schließlich sei noch angemerkt, dass die Erkenntnis, wonach bei der empirischen Innovationsforschung mehr auf die Ursachen der in der ersten Neo-Schumpeter-Hypothese postulierten technologischen Überlegenheit der Großunternehmen abgestellt werden muss, nicht zur Folge hat, dass die Verwendung der Unternehmensgröße als einer der erklärenden Variablen gemieden werden soll. So zeigt Arvanitis (1997), dass der Einsatz eines Maßes der Unter-

140 Zur modelltheoretischen Behandlung der dargestellten Zusammenhänge vgl. Cohen/Klepper (1996a) und Cohen/Klepper (1996b).

141 Vgl. Arvanitis (1997), S. 473. 
nehmensgröße (Zahl der Beschäftigten in der quadratischen Spezifikation) die Aussagekraft der Modelle zur Erklärung der Forschungs- und Innovationstätigkeit im Wirtschaftssektor eindeutig steigert. ${ }^{142}$ Der Autor schlussfolgert, dass die Unternehmensgröße als diejenige Variable fungieren kann, die die sonst im Modell nicht berücksichtigten unternehmensspezifischen Effekte aufgreifen kann.

\subsubsection{Marktstruktur und Forschungstätigkeit}

Bei der Diskussion der zweiten Neo-Schumpeter-Hypothese, die die Beziehung zwischen Marktstruktur und Forschungstätigkeit problematisiert, gilt es zwei verschiedene Zusammenhänge zu unterscheiden. ${ }^{143}$ Zum einen kann davon ausgegangen werden, dass Wirtschaftsakteure FuE-Anstrengungen in der Erwartung einer temporären Monopolstellung unternehmen. Die Monopolsituation - wie sie auch durch die Gewährung der Patentrechte an kommerziell verwertbaren Erfindungen entsteht - ermöglicht es den Unternehmen, sich Forschungserträge anzueignen und überdurchschnittliche Gewinne zu realisieren. Die Aussicht auf diese Marktstärke bzw. - in der Terminologie der Innovationsforschung - die ex-post Marktmacht stellt einen der wichtigsten Innovationsanreize dar und bildet die notwendige Voraussetzung für den technischen Fortschritt. ${ }^{144}$

Zum anderen wird in der in der Tradition Schumpeters stehenden Innovationsökonomik angenommen, dass vorhandene Marktkonzentration ex-ante innovationsfördernd wirkt,

- da es für das jeweilige Unternehmen eine stabile Marktsituation schafft, in der das Verhalten der Wettbewerber zuverlässig prognostiziert werden kann und exzessive, in ungleich höherer externer Unsicherheit resultierende Konkurrenz vermieden wird $^{145}$,

142 Vgl. Arvanitis (1997), S. 478.

${ }^{143} \mathrm{Zu}$ der Notwendigkeit dieser Unterscheidung vgl. Scherer (1992), S. 1418, Cohen (1995), S. 191 f., Schmidt/Elßer (1990), S. 560, den Hertog/Thurik (1993), S. 283.

$144 \mathrm{Vgl}$. dazu die Aussage von Arrow (1962), S. 622: „The only ground for arguing that monopoly may create superior incentives to invent is that appropriability may be greater under monopoly than under competition."

145 Vgl. hierzu die Anmerkung von Schumpeter (1942/1987), S. 168: „Der Hauptwert, den die durch Patente oder monopolistische Strategie gesicherte Stellung des <einzigen Verkäufers $>$ für einen Konzern hat, besteht nicht so sehr in der Möglichkeit, sich zeitweilig 
- weil in oligopolistischer Marktstruktur der Innovationswettbewerb gegenüber dem Preiswettbewerb an Bedeutung gewinnt, und

- weil sie den Unternehmen ermöglicht, Renten zu realisieren, welche die interne Finanzierung der Forschungsvorhaben wesentlich erleichtern. ${ }^{146}$

Im Rahmen der auf Schumpeter zurückgehenden Innovationsökonomik kann folglich ein Postulat der Wechselbeziehung zwischen der Größe eines Unternehmens im Markt und seiner Forschungstätigkeit abgeleitet werden: Einerseits wirkt sich vorhandene Marktkonzentration positiv auf die Forschungstätigkeit der auf dem Markt agierenden Unternehmen aus. Andererseits unternehmen gewinngesteuerte Wirtschaftseinheiten FuE-Anstrengungen in der Erwartung einer künftigen Monopolstellung, d.h. Forschungstätigkeit von Unternehmen führt zur Entstehung von Monopolen. Trotz der postulierten wechselseitigen Beziehung wurde in der früheren empirischen Forschung vorwiegend ein Teilaspekt untersucht: der Einfluss der (ex-ante) bestehenden Marktstruktur auf das Innovationsverhalten. ${ }^{147}$ In Regressionsanalysen wurden folglich verschiedene Maße der Marktkonzentration als exogen gegebene Größen zur Erklärung der Variation der Forschungsintensitäten in einzelnen Branchen herangezogen. Diese Vorgehensweise führte jedoch zu keinen eindeutigen Ergebnissen; sowohl positive als auch negative Zusammenhänge sind ermittelt worden. ${ }^{148}$

In einigen Studien wurde ein nichtlinearer funktionaler Zusammenhang zwischen der Marktstruktur und dem Innovationsverhalten der Unternehmen untersucht. Die Hypothese einer nichtlinearen Beziehung wurde folgendermaßen begründet. Einerseits kann die Forschungsintensität in Konkurrenzmärkten gering ausfallen, da unter den Bedingungen des vollkommenen Wettbewerbs die Erzielung jeglicher Monopolrenten unmöglich und folglich die Finanzierung der FuE-Tätigkeit erschwert wird. Andererseits unterbleibt in Monopolmärkten die Anreizwirkung freier Konkurrenz. Ferner kann argumentiert werden,

gemäß dem monopolistischen Schema zu verhalten, als vielmehr im Schutz, den sie gegen eine zeitweilige Desorganisation des Marktes gewährt, und in der Zeitspanne, die sie für eine langfristige Planung sichert."

146 Vgl. den Hertog/Thurik (1993), S. 283.

$147 \mathrm{Vgl}$. Cohen (1995), S. 192. Auf lediglich zwei Ausnahmen aus den 60er und 70er Jahren weisen Levin und Reiss hin, vgl. Levin/Reiss (1984), S. 175.

148 Vgl. auch dazu Übersichtsstudien wie Frisch (1993), S. 52-69, Cohen (1995), S. 192, Gotschalk/Janz (2003), S. 27 f., Symeonidis (1996), S. 11-16, Kamien/Schwartz (1975), S. 20-22. 
dass sich ein Monopolist gegen eine Innovation entscheiden kann, um bestehende Kapitalwerte bzw. Monopolrenten zu schützen. ${ }^{149}$ Mit der Zulassung eines nichtlinearen Zusammenhanges wurde auch den Ergebnissen einer Analyse von Arrow (1962) Rechnung getragen, in der Innovationsanreize unter alternativen Marktstrukturen (vollkommener Wettbewerb und Monopol) bei Voraussetzung perfekter Aneignung der Forschungserträge untersucht wurden und gezeigt werden konnte, dass sie in einer durch Wettbewerb im Produktionsbereich gekennzeichneten Marktstruktur höher als bei einem Angebotsmonopolisten ausfallen. ${ }^{150}$

In mehreren älteren Studien ist Evidenz für den erwarteten inversen U-förmigen Zusammenhang zwischen Wettbewerbs- und Forschungsintensität gefunden worden. Das Maximum lag bei einem Umsatzanteil der größten vier Unternehmen einer Branche von 50 bis $65 \%{ }^{151}$ Allerdings erwies sich dieser Zusammenhang als stark empfindlich gegenüber der Wahl der verwendeten Kontrollvariablen. Die Einführung von festen Firmen- und Brancheneffekten ${ }^{152}$ bzw. die Berücksichtigung von Variablen, die für die interindustriellen Unterschiede in Aneignungsbedingungen und technologischen Möglichkeiten kontrollieren sollten $^{153}$, führte zu statistisch insignifikanten Koeffizienten bei den Konzentrationsvariablen. Die Schlussfolgerung, dass der Einfluss der Marktkonzentration nicht mehr feststellbar ist, wenn verstärkt für Unternehmens- und Branchencharakteristika kontrolliert wird, ist durch die Ergebnisse neuerer Studien bestätigt. In den Studien von Felder et al. (1996) sowie Becker und Peters (2005) zeigt sich kein signifikanter Einfluss der Marktkonzentration auf die Forschungsintensität der deutschen Unternehmen. ${ }^{154}$ Kein signifikanter Einfluss der Marktkonzentration auf die Wahrscheinlichkeit für die Aufnahme der FuE-Tätigkeit wird in den Untersuchungen von Braga und Willmore (1991), Kumar und Saqib (1996) festgestellt, ${ }^{155}$ in der Studie von Becker und Peters

${ }^{149}$ Zur kritischen Auseinandersetzung mit diesem Argument vgl. Schumpeter (1942/1987), S. $157 \mathrm{f}$.

150 Vgl. Arrow (1962), S. 619 ff., für die Diskussion des Modells von Arrow vgl. Dasgupta/Stiglitz (1980), S. 269 ff. und Baldwin/Scott (1987), S. 14 f.

151 Vgl. Levin/Cohen/Mowery (1985), S. 21, Scott (1984), S. 235, Scherer (1967), S. 530; Kamien und Schwartz berichten auch von einer weiteren (unveröffentlichten) Untersuchung von Kelly, in der das gleiche Ergebnis beobachtet wurde, vgl. Kamien/Schwartz (1975), S. 21.

152 Vgl. Scott (1984), S. 235.

153 Vgl. Levin/Cohen/Mowery (1985), S. 23.

154 Vgl. Felder/Licht/Nerlinger/Stahl (1996), S. 142, Becker/Peters (2005), S. 141.

155 Vgl. Braga/Willmore (1991), S. 428, Kumar/Saqib (1996), S. 718. 
(2005) zeigt sich dagegen ein positiver Einfluss der Marktkonzentration auf die FuE-Aktivität der Unternehmen.

Auf zwei Schwächen der empirischen Tests der NSH II soll an dieser Stelle hingewiesen werden. Die erste Schwäche begründet sich in der Annahme der Exogenität der Marktstruktur. ${ }^{156}$ Dieser Nachteil wurde in der innovationsökonomischen Literatur relativ früh erkannt und führte zur modelltheoretischen Behandlung der wechselseitigen Zusammenhänge zwischen Marktstruktur und Forschungstätigkeit. ${ }^{157}$ Auf der Grundlage dieser Modelle sind empirische Untersuchungen durchgeführt worden, in denen Forschungsintensitäten und Marktstruktur simultan endogenisiert wurden. So zeigte eine frühe Studie von Levin und Reiss (1984) einen marktkonzentrationsinduzierenden Effekt von industrieller Forschung und Entwicklung und negative Auswirkungen der Marktkonzentration auf die Forschungsintensität. Dieses Ergebnis wurde in den letzten Jahren von Gottschalk und Janz (2001) anhand von Daten für die deutsche Industrie bestätigt. Die Autoren verknüpften im Rahmen eines simultanen Modells Marktkonzentrationsdaten mit den Daten zu den FuEIntensitäten der Branchen des Verarbeiteten Gewerbes und konnten zeigen, dass sich die Forschungstätigkeit der Unternehmen langfristig auf die Erhöhung der Marktkonzentration auswirkt, während Marktkonzentration zur Senkung der FuE-Intensität führt.

Als zweite Schwäche der Auseinandersetzung mit der NSH II kann die Herleitung der Marktkonzentrationsmaße auf relativ hoher Aggregationsebene aufgefasst werden. Dies kann als Nachteil eingestuft werden, da davon ausgegangen werden kann, dass die auf Basis einer zweistelligen Wirtschaftszweigklassifikation abgeleitete Umsatzkonzentrationsrate nur sehr grob die für eine einzelne Unternehmung relevante Marktstruktur abbildet. Darüber hinaus sind auch die auf tieferer Disagreggationsebene hergeleiteten Umsatzkonzentrationsdaten nicht zwangsläufig gut geeignet, die relevante Marktstruktur der auf den internationalen Märkten agierenden Unternehmen zu beschreiben. ${ }^{158}$ Daher wird in ausgewählten neueren Untersuchungen versucht, die Marktstruktur auf der Unternehmensebene zu erfassen, z.B. unter Zuhilfenahme der einzelwirtschaftlichen Angaben zur Anzahl der weltweiten Wettbewerber: Eine geringe Anzahl der Wettbewerber reflektiert demnach Oligopolmacht. Der wichtigste Vorteil

156 Vgl. Gottschalk/Janz (2003), S. 28.

157 Für eine modelltheoretische Betrachtung des Zusammenhangs zwischen Marktkonzentration und Forschungstätigkeit vgl. z.B. Levin/Reiss (1984), sowie die dort zitierte Literatur.

158 Vgl. hierzu die Anmerkung von Arvanitis/Hollenstein (1996), S. 21. 
der auf diese Weise gewonnenen Konzentrationsvariablen liegt darin, dass dank dieses Indikators die Marktsituation der auf einem "Nischenmarkt" agierenden Klein- und Mittelunternehmen adäquat wiedergegeben werden kann. ${ }^{159}$

Untersuchungen, in denen die Marktstruktur auf diese Weise erfasst wird, liefern jedoch ebenfalls keine robusten Ergebnisse. Zwar kann bei Betrachtung der Ergebnisse von Arvanitis und Hollenstein (1996) sowie Arvanitis und von Arx (2004) ein schwacher Hinweis auf eine positive Beziehung zwischen Marktkonzentration und Forschungstätigkeit gefunden werden. Allerdings ist der postulierte positive Zusammenhang nicht durchgehend (d.h. unabhängig von Schätzverfahren und FuE-Indikatoren) signifikant. In der Untersuchung von Arvanitis und Hollenstein (1994) erweist sich der Einfluss der Marktkonzentration nur in der Gruppe der Produktinnovatoren signifikant. ${ }^{160}$ In der Studie von Lenz (1998) sind die Konzentrationsvariablen bei der Berücksichtigung von Innovatoren und Nichtinnovatoren nicht signifikant; nur in der OLS-Schätzung der Forschungsaufwendungen bzw. FuE-Intensität der Innovatoren zeigt sich der Einfluss der mittleren Marktkonzentration signifikant positiv. ${ }^{161}$

\subsection{Unternehmensinterne Determinanten der FuE-Tätigkeit}

\subsubsection{Finanzierung}

Die empirische Evidenz zu den finanziellen Rahmenbedingungen und ihren Auswirkungen auf die Forschungstätigkeit im Wirtschaftssektor ist relativ umfangreich, da Hinweise auf die Problematik der Finanzierung von FuEProjekten bereits bei Schumpeter gefunden werden können. ${ }^{162}$ Schumpeter sah einen der Hauptgründe für die technologische Überlegenheit von großen marktmächtigen Unternehmen in ihrer Möglichkeit, die vorhandenen Monopolgewinne für die Finanzierung von FuE aufwenden zu können. Die in der

159 Für einen alternativen Vorschlag eines Indikators der Marktstruktur: der Unternehmensdichte (firm density), definiert als Anzahl der Firmen bezüglich der Marktgröße, vgl. Lee (2003b).

$160 \mathrm{Vgl}$. Arvanitis/Hollenstein (1994a), Tabellen 4a und 4b, S. 25 ff.

$161 \mathrm{Vgl}$. Lenz (1998), Tabellen 7.6 und 7.8, S. $134 \mathrm{ff}$.

162 Vgl. z.B. Schumpeter (1942/1987), Kap. 8. 
Tradition Schumpeters stehende Innovationsökonomik betonte folglich die Bedeutung der ex-ante Profitabilität eines Unternehmens für seine Entscheidungen im FuE-Bereich. So argumentieren beispielweise Kamien und Schwartz (1978):

„Among the leading characteristics commonly associated with industrial research and development, one of the most prominent is the virtual necessity for it to be financed internally from a firm's current profits and accumulated funds." 163

Richtungsweisend für die Auseinandersetzung mit den Fragen der Finanzierung von Forschung und Entwicklung war auch ein späterer Beitrag von Arrow (1962). Arrow identifizierte zwei Störungen im Bereich der Finanzierung von FuE. Zum einen erkannte er die Schwierigkeit bei der Aneignung des in FuEProzessen erarbeiteten Wissens, das aufgrund seiner Eigenschaft als öffentliches Gut einer unkontrollierten Diffusion unterliegen kann. Die daraus folgende begrenzte Möglichkeit der Unternehmen, sich Erträge eigener Forschungstätigkeit anzueignen, wirke demnach negativ auf den Anreiz, in FuE zu investieren. Die Frage nach der Bedeutung der sogenannten Aneignungs- bzw. Appropriierungsbedingungen (appropriability conditions) wird Gegenstand der Untersuchung in Abschnitt 3.4.2 sein. Zum anderen sprach jedoch Arrow auch die Störungen an, die entstehen, wenn externe Mittel zur Finanzierung von FuEVorhaben herangezogen werden müssen. ${ }^{164}$

Aufbauend auf der Analyse von Arrow ging die spätere innovationsökonomische Literatur der Frage nach den Ursachen für die relative Vorteilhaftigkeit der Innenfinanzierung von FuE nach. Folgende zwei primären Ursachen potenzieller Unterschiede zwischen den Kosten des Eigen- und Fremdkapitals im Bereich der Finanzierung von Forschung und Entwicklung wurden identifiziert: ${ }^{165}$

Zum einen handelt es sich bei FuE um einen Vorgang, bei dem das immaterielle Gut Wissen unter relativ intensivem Einsatz von qualifizierter Arbeitskraft produziert wird. ${ }^{166}$ Die für die Finanzierung von FuE-Vorhaben benötig-

163 Kamien/Schwartz (1978), S. 252.

164 Vgl. Hall (2002), S. 35 f.

${ }^{165}$ Für die Erläuterung weiterer Ursachen, wie 1) moral hazard-Problematik, die aufgrund der Trennung von Eigentum und Management entsteht und 2) landesspezifischer Steuerpolitik vgl. Hall (2002), S. 38-41. Für die Analyse der Problematik von moral hazard vgl. auch Arrow (1962), S. 612 ff. Zur Behandlung der Agency-Problematik im Bereich der Finanzierung von FuE vgl. auch Haid/Weigand (2001).

166 Hall geht von einem durchschnittlichen Anteil der Personalkosten am FuE-Budget von über $50 \%$ aus, vgl. Hall (2002), S. 36 . 
ten Ressourcen werden i.d.R. zum großen Teil für Personalkosten aufgewandt. Im Unterschied zu herkömmlichen (Sachkapital-)Investitionen sind bei FuEInvestitionsprojekten folglich kaum tangible Vermögenswerte involviert: die FuE-Aufwendungen müssen zum großen Teil als sunk costs eingestuft werden. Dies hat Auswirkungen auf die Verfügbarkeit von Sicherheiten, und, in der Folge, auf die Kosten der externen Finanzierung. ${ }^{167}$

Zum anderen wird auf die Bedeutung von den im FuE-Bereich besonders ausgeprägten Informationsasymmetrien hingewiesen. ${ }^{168}$ Hierbei wird folgendenmaßen argumentiert: Da die FuE-betreibenden Unternehmen über bessere Informationen bezüglich der Erfolgswahrscheinlichkeit der FuE-Projekte informiert sind, entsteht das klassische "lemons"-Problem. ${ }^{169}$ Die externen Kapitalgeber sind nicht in der Lage, die erfolgversprechenden Projekte von den hochriskanten Vorhaben zu unterscheiden und berechnen daher einen lemonsAufschlag. ${ }^{170}$ Folgt man nun der Arrowschen Konzeptualisierung vom Wissen als einem öffentlichen Gut und geht von dem bereits im einleitenden Kapitel beschriebenen "Informationsparadoxon" aus ${ }^{171}$, so kommt man zu dem Ergebnis, dass der Aufschlag im FuE-Bereich höher als im Bereich der tangiblen Investitionen ausfallen muss, weil die Unternehmer wenige Anreize zur Offenlegung von innovationsrelevanten Informationen haben. ${ }^{172}$ Himmelberg und Petersen (1994) argumentieren, dass strategische Abwägungen die Unternehmen eher dazu veranlassen können, die Informationsasymmetrien aufrecht zu erhalten $^{173}$, um die mit dem innovationsrelevanten Wissen verbundenen Wettbewerbsvorteile zu sichern und die Diffusion dieses Wissens zu den Wettbewerbern $\mathrm{zu}$ vermeiden.

Frühere empirische Evidenz zum Einfluss des höheren Innenfinanzierungspotenzials auf die Forschungsintensitäten der Unternehmen wurde in den Studien von Kamien und Schwartz (1982), Frisch (1993) sowie Cohen und Levin (1989) zusammengefasst; alle Autoren kamen zu dem Schluss, dass die Empirie keinen eindeutigen Beweis für den positiven Zusammenhang zwischen der Ertragskraft

167 Vgl. dazu Kamien/Schwartz (1978), S. 252, Himmelberg/Petersen (1994), S. 40.

168 Vgl. Hall (2002), S. 38, Czarnitzki (2002), S. 1., Himmelberg/Petersen (1994), S. 39 f.

$169 \mathrm{Vgl}$. Akerlof (1970).

$170 \mathrm{Vgl}$. Hall (2002), S. 38.

$171 \mathrm{Vgl}$. Abschnitt 2.3.1.

172 Vgl. hierzu Czarnitzki (2002), S. 1, Hall (2002), S. 38.

173 Vgl. Himmelberg/Petersen (1994), S. 39. 
eines Unternehmens und seiner FuE-Tätigkeit liefert. ${ }^{174}$ Es kann jedoch vermutet werden, dass die festgestellte Uneindeutigkeit der Ergebnisse aus drei Schwächen der früheren Forschung resultiert. Erstens: Kamien und Schwartz (1982) weisen darauf hin, dass die Profitabilität einer Unternehmung eher die Rolle eines Schwellenfaktors (threshold factor) spielen könnte: Zwar bildet das Innenfinanzierungspotenzial eine Voraussetzung für die Aufnahme der FuETätigkeit; beim Überschreiten einer bestimmten Schwelle führt jedoch eine weitere Steigerung der Ertragskraft einer Unternehmung nicht zwangsläufig zur Ausweitung der Forschungstätigkeit. ${ }^{175}$ Zweitens betonen Himmelberg und Petersen (1994), dass die älteren Untersuchungen auf Datensätzen basieren, die lediglich Großunternehmen umfassen. Da diese Unternehmen jedoch insgesamt ein höheres Innenfinanzierungspotenzial aufweisen, kann davon ausgegangen werden, dass sie nicht zu der Gruppe der finanzierungsbeschränkten Unternehmen gehören. ${ }^{176}$ Drittens wird auf die Konsequenzen der immanenten Eigenschaften der FuE-Tätigkeit für die Methodologie der empirischen Forschung hingewiesen. Erfolgreiche wie erfolglose FuE führe demnach zu einer Ausweitung der firmeninternen Wissensbasis, die sich zum erheblichen Teil in den Erfahrungswerten der Mitarbeiter begründet und somit prozessualer Natur und personengebunden sein kann. Um diese Wissensbasis aufrechtzuerhalten und insbesondere den personengebundenen Transfer dieses wettbewerbsrelevanten Wissens zu den Konkurrenten zu vermeiden, bemühen sich die Unternehmen, das FuE-Budget über mehrere Perioden zu glätten und nicht an die Schwankungen des Kapitalflusses zu koppeln. Die fehlende Kopplung der FuEAufwendungen an die periodenabhängige Änderung der Ertragskraft, bzw. - in Worten der Innovationsforschung - die hohen Anpassungskosten (adjustment cost) der FuE-Tätigkeit - hat Konsequenzen für die Spezifikation der ökonometrischen Modelle, denen in der früheren Forschung u.U. nicht in ausreichendem Maße Rechnung getragen wurde. ${ }^{177}$

Die Methodologie und die Ergebnisse der neueren Forschung zur Finanzierung von FuE wurden von Hall (2002) zusammengefasst. Hall kommt zur Schlussfolgerung, dass sich eine Finanzierungslücke in der Gruppe der großen, etablierten Unternehmen nicht feststellen lässt, obwohl davon auszugehen ist, dass die Innenfinanzierung im Vergleich zur Kreditfinanzierung bevorzugt wird. Gleich-

${ }^{174}$ Vgl. Kamien/Schwartz (1982), S. 95-98, Frisch (1993), S. 93-102, Cohen/Levin (1989), S. 1072 .

175 Vgl. hierzu Kamien/Schwartz (1982), S. 98.

176 Vgl. Himmelberg/Petersen (1994), S. 39.

177 Vgl. Hall (2002), S. 36. 
zeitig jedoch findet er Hinweise dafür, dass kleine bzw. neugegründete Unternehmen (start-ups) in forschungsintensiven Industriezweigen Finanzierungsbeschränkungen unterliegen können. ${ }^{178}$

\subsubsection{Diversifizierung}

Die explizite Formulierung der Hypothese, die technologische Überlegenheit der Großunternehmung hänge mit ihrer Fähigkeit zur Diversifizierung zusammen, lässt sich auf Nelson (1959) zurückverfolgen. Allerdings argumentierte Nelson in seinem Beitrag lediglich, dass die Diversifizierung eine stärkere Neigung zur Grundlagenforschung hervorrufen könne. Für die vorliegende Arbeit ist eine spätere Hypothese von größerer Bedeutung, die nicht explizit auf die Grundlagenforschung abzielt, sondern den Zusammenhang zwischen allgemeiner Forschungstätigkeit und Diversifizierung problematisiert. Die Begründung für die stärkere Forschungsneigung bzw. größere Forschungsintensität diversifizierter Unternehmen läge darin, dass bei diesen Unternehmen der erwartete Ertrag von Investitionen in Forschung und Entwicklung aufgrund der höheren unternehmensinternen Anwendungswahrscheinlichkeit von Zufallsentdeckungen höher ausfällt. ${ }^{179}$

Die empirischen Studien zur Diversifizierungshypothese fingen mit der Arbeit von Scherer (1965) an; eine Zusammenstellung der Ergebnisse älterer Studien und eine genaue Besprechung der im Einzelnen verwendeten Variablen und Schätzverfahren kann bei Frisch (1993), Symeonidis (1996) sowie Kamien und Schwartz (1975) gefunden werden. Alle Autoren kommen nach einer eingehenden Analyse zu dem Ergebnis, dass die Hypothese, Diversifizierung wirke sich positiv auf die Forschungsintensität der Unternehmen aus, als weder bestätigt noch widerlegt angesehen werden muss. ${ }^{180}$

Der Einfluss der Diversifizierung auf die Entscheidungen im FuE-Bereich wird auch in neueren Studien überprüft. Felder et al. (1996) berücksichtigen ein Diversifikationsmaß in ihrer Studie zu den Bestimmungsgründen der Forschungstätigkeit, in der Daten zur Innovationstätigkeit deutscher Unternehmen ausgewertet werden. Als Diversifizierungsmaß verwenden sie dabei den Kehr-

178 Vgl. Hall (2002), S. 48 und S. 45.

179 Vgl. Frisch (1993), S. 85.

180 Vgl. Frisch (1993), S. 85-91, Symeonidis (1996), S. 22, Kamien/Schwartz (1975), S. 27. 
wert des Herfindal-Indexes der Produktgruppenumsätze. ${ }^{181}$ Die Ergebnisse lassen darauf schließen, dass stärkere Diversifizierung mit einer erhöhten Wahrscheinlichkeit für die Aufnahme der FuE-Tätigkeit einhergeht, jedoch keinen signifikanten Einfluss auf die Forschungsintensität der Unternehmen ausübt. ${ }^{182}$ Einen positiven Einfluss der Diversifizierung auf die Entscheidung für FuE stellen auch Becker und Peters (2005) fest, die ebenfalls Daten des Mannheimer Innovationspanels verwenden. In dieser Studie erweist sich jedoch der Einfluss der Diversifizierung auf die Forschungsintensität der Unternehmen nur dann signifikant positiv, wenn Forschungsintensität mit dem logarithmierten Anteil des FuE-Personals an der Gesamtzahl der Beschäftigten gemessen wird. ${ }^{183}$ In einer Studie von Becker und Dietz (2002), in der FuE-Intensität und Kooperationstätigkeit der deutschen Unternehmen simultan geschätzt werden, ist hingegen in drei von vier Fällen kein Einfluss der Diversifizierung auf die FuEIntensität feststellbar.

Crepon et al. (1996) verwenden ebenfalls den Kehrwert des Herfindal-Index als ein Maß des Diversifikationsgrades einer Unternehmung und untersuchen ihren Einfluss auf die Forschungstätigkeit französischer Unternehmen. Diversifizierung beeinflusst dabei in positiver Weise die Entscheidung für FuE, jedoch führt sie nicht zwangsläufig zur Ausweitung der FuE-Tätigkeit. ${ }^{184}$ Auch in der Studie von Braga und Willmore (1991) zur Innovationstätigkeit brasilianischer Unternehmen beeinflusst Diversifizierung in positiver Weise die Entscheidung für eine Aufnahme von FuE-Tätigkeit. ${ }^{185}$ In der Unterschung von Veugelers (1997) zeigt sich kein signifikanter Einfluss der Diversifizierung auf die Forschungstätigkeit belgischer Unternehmen. ${ }^{186}$ In der Studie von Licht und Zoz (2000), in der Determinanten der Patentaktivität deutscher Unternehmen untersucht werden, zeigt sich kein signifikanter Einfluss der Diversifizierung auf die Patentneigung der Unternehmen. Ferner lassen die Ergebnisse der Schätzung darauf schließen, dass eine stärkere Diversifizierung zur Senkung der Anzahl von Patentanträgen führt. ${ }^{187}$

181 Zur Verfügung standen den Autoren Angaben zu Umsatzanteilen der vier wichtigsten Produktgruppen der jeweiligen Unternehmen, vgl. Felder/Licht/Nerlinger/Stahl (1996), S. $137 \mathrm{f}$.

182 Vgl. Felder/Licht/Nerlinger/Stahl (1996), S. 142.

183 Vgl. Becker/Peters (2005), S. 141.

184 Vgl. Crepon/Duguet/Kabla (1996), S. 77.

185 Vgl. Braga/Willmore (1991), S. 428.

$186 \mathrm{Vgl}$. Veugelers (1997), S. 310.

187 Vgl. Licht/Zoz (2000), S. 324. 
Insgesamt liefern die neueren Untersuchungen einen Hinweis darauf, dass Diversifizierung die einzelwirtschaftliche Entscheidung für FuE begünstigt, jedoch nicht zwangsläufig zu einer höheren Forschungsintensität der Unternehmen führt.

\subsubsection{Exporttätigkeit}

Die Exportintensität einer Unternehmung wird in der neueren empirischen Innovationsforschung häufig als erklärende Variable herangezogen. Zur Rechtfertigung der Berücksichtigung dieser erklärenden Variable wird meist auf die Bedeutung der Exporte als ein Instrument der Absatzsteigerung hingewiesen: Da Forschungstätigkeit mit erheblichen Fixkosten verbunden ist, führt die Ausweitung der Absatzmärkte zur Senkung der Durchschnittskosten von FuE und ermöglicht somit die Ausweitung der Innovationstätigkeit. ${ }^{188}$ Eine alternative Begründung für die Berücksichtigung der Exporttätigkeit als einer der Determinanten der Patentneigung von Unternehmen schlagen in ihrer empirischen Studie Licht und Zoz (2000) vor. Sie argumentieren, dass exportierende Unternehmen auf eine größere Anzahl von Wettbewerbern treffen und daher stärkere Anreize zum Schutz innovationsbedingter Wettbewerbsvorteile haben dürften. ${ }^{189}$ Auch bei dieser Begründung wird ein positiver Einfluss der Exportauf die Forschungstätigkeit erwartet.

In der Tat zeigt sich in der Empirie, dass sich die Exporttätigkeit auf die Forschungsneigung bzw. auf die Forschungsintensität der Unternehmen signifikant positiv auswirkt. ${ }^{190}$ Auch in der Studie von Licht und Zoz (2000) zu den Determinanten der Patenttätigkeit wird bestätigt, dass eine höhere Exportintensität einen positiven Einfluss auf die Patentaktivitäten von Unternehmen ausübt. ${ }^{191}$

Problematisch erscheint allerdings, dass viele Studien der Kausalitätsrichtung zwischen der Export- und Forschungstätigkeit keine Beachtung schenken. ${ }^{192}$

\footnotetext{
188 Vgl. Kleinknecht/Oostendorp (2002), S. 310.

189 Vgl. Licht/Zoz (2000), S. 317.

190 Vgl. Leiponen (2002), Tabelle A11.1, S. 271, Nooteboom/Vossen (1995), Felder/Licht/Nerlinger/Stahl (1996), S. 141 f., Arvanitis/von Arx (2004), Tab. 5.4-5.7, S. 42 ff., Braga/Willmore (1991), S. 428, Kumar/Saqib (1996), S. 718, Becker/Peters (2005), S. 141, Becker/Dietz (2002), S. 26, Siddharthan/Agarwal (1992), S. 106, Lee (2003a), S. 392 f., Lee (2003b), S. $151 \mathrm{ff}$.

191 Vgl. Licht/Zoz (2000), S. 326.

$192 \mathrm{Vgl}$. Kleinknecht/Oostendorp (2002), S. $310 \mathrm{f}$.
} 
Dabei ist es durchaus denkbar, dass erst durch Erfolge in der Forschungstätigkeit firmenspezifische Vorteile entstehen, die es dem Unternehmen ermöglichen, sich auf internationalen Märkten zu behaupten. ${ }^{193}$ Eine Untersuchung von Kleinknecht und Oostendorp (2002) zum Zusammenhang zwischen FuEund Exporttätigkeit bestätigt das Ergebnis, dass die Exporttätigkeit einen signifikant positiven Einfluss auf die Forschungstätigkeit ausübt. Es zeigt sich jedoch auch, dass die Forschungstätigkeit die Wahrscheinlichkeit für eine Exporttätigkeit erhöht.

\subsubsection{Konzernzugehörigkeit}

In mehreren neueren Studien, die bei der Suche nach Bestimmungsgründen der FuE-Tätigkeit verstärkt für den Einfluss von Unternehmenseigenschaften kontrollieren, wird der Zusammenhang zwischen Konzernzugehörigkeit und Forschungsaktivität untersucht. Hierbei wird argumentiert, dass in Unternehmensgruppen bzw. Konzernen die FuE-Tätigkeit oft zentralisiert ist und folglich in einzelnen Unternehmen nur in geringem Umfang stattfindet bzw. vollständig aufgegeben wird. ${ }^{194}$ Daher wird erwartet, dass sich die Zugehörigkeit zu einer Unternehmensgruppe negativ auf die FuE-Tätigkeit der Tochtergesellschaften auswirkt.

Das Postulat eines negativen Zusammenhangs wird jedoch in der Empirie nicht bestätigt. In 1) der auf finnischen Unternehmensdaten basierenden Untersuchung von Leiponen (2002), 2) der auf italienischen Daten basierenden Studie von Evangelista et al. (1997) und 3) der auf niederländischen Daten fußenden Studie von Kleinknecht und Oostendorp (2002) hat die Zugehörigkeit zu einer Unternehmensgruppe bzw. einem Konzern eine erhöhte Wahrscheinlichkeit für die Aufnahme der FuE-Tätigkeit zur Folge. ${ }^{195}$ In der auf deutschen Unternehmensdaten basierenden Studie von Felder et al. (1996) erweist sich der Einfluss der Konzernzugehörigkeit dagegen als nicht signifikant. ${ }^{196}$

193 Vgl. Kumar/Saqib (1996), S. 715.

194 Vgl. Felder/Licht/Nerlinger/Stahl (1996), S. 139.

195 Vgl. Leiponen (2002), S. 271, Evangelista/Perani/Rapiti/Archibugi (1997), S. 526, Kleinknecht/Oostendorp (2002), S. 313.

196 Vgl. Felder/Licht/Nerlinger/Stahl (1996), S. 141 f. 


\subsection{Industrieökonomische Determinanten der FuE-Tätigkeit}

Die empirischen Studien zum Zusammenhang zwischen Marktkonzentration und Forschungstätigkeit lieferten einen Hinweis darauf, dass bei der Untersuchung der Einflussgrößen auf die FuE im Wirtschaftssektor stärker die branchenspezifischen Eigenschaften der Technologie berücksichtigt werden sollten. So wiesen Cohen und Levin darauf hin, dass die Varianz der Marktkonzentrationsmaße allein nur einen geringen Anteil der Varianz der Forschungsintensitäten erklären konnte. Dagegen führte die Berücksichtigung von Variablen, welche die industriespezifische Nachfrage, technologische Möglichkeiten und Appropriierungsbedingungen abbilden sollten, zu einer erheblichen Steigerung des Anteils der erklärten Varianz. ${ }^{197}$

Auch in den theoretischen Modellen, die Forschungstätigkeit und Marktstruktur endogenisieren, wurde die Bedeutung dieser drei Variablen anerkannt. So behaupteten Levin und Reiss:

"[T]he relation of $R \& D$ and market structure must be explained by an appeal to more fundamental factors that jointly determine concentration and R\&D: the structure of demand; the richness of technological opportunities; and the technological and institutional conditions governing appropriability. “ 198

Sowohl in der empirischen Forschung, als auch in der theoretischen Modellbildung sind damit branchenspezifische Rahmenbedingungen sowie Eigenschaften der Technologie als potenzielle Einflussgrößen auf die Forschungsintensität im Wirtschaftssektor herausgestellt worden. Die empirische Evidenz zu der Bedeutung dieser Eigenschaften als Determinanten der FuE-Aktivitäten im Wirtschaftssektor sollen in den folgenden Abschnitten näher betrachtet werden.

\subsubsection{Nachfrage}

Auf die Bedeutung der Nachfrage als einen der Bestimmungsgründe der Forschungstätigkeit im Wirtschaftssektor hat bereits in den 60er Jahren Schmook-

$197 \mathrm{Vgl}$. Cohen/Levin (1989), S. $1077 \mathrm{f}$.

198 Levin/Reiss (1984), S. 175 f. 
ler hingewiesen. ${ }^{199}$ Schmookler kritisierte die Einschätzung, nach der Innovationen von der Grundlagenforschung begünstigt würden und anschließend ihre eigene Nachfrage schafften. Er betonte vielmehr die aktive Rolle der Konsumenten: Die Änderung von Konsumentenpräferenzen führt zu einer Verschiebung von Gewinnchancen und löst Anpassungsprozesse bei Unternehmen aus. ${ }^{200}$ Die Initiierung bestimmter Inventionen wird Marktimpulsen verdankt, die durch diejenigen Mitarbeiter ins Unternehmen eingebracht werden, die in direktem Kundenkontakt stehen und somit die Marktentwicklung genau verfolgen können. Den FuE-Abteilungen kommt in der unternehmensinternen Arbeitsteilung nur eine reaktive Rolle zu; sie sind dafür zuständig, Produkte bzw. Prozesse zu entwickeln, die die durch die Kunden bereits erkannten Bedürfnisse befriedigen sollen - und nicht dafür, durch Erfindung der Neuerungen neue Bedürfnisse zu schaffen. ${ }^{201}$

Die Ausführungen von Schmookler lösten die Debatte aus, ob Innovationen primär durch Nachfrage induziert (sog. market-pull bzw. demand-pull Hypothese) oder grundsätzlich durch allgemeine Fortschritte in der Technologie begünstigt werden (sog. technology-push bzw. supply-push Hypothese). In der empirischen Forschung führte dies schließlich zur Untersuchung von zwei Fragestellungen. Zum einen wurde versucht $\mathrm{zu}$ ermitteln, inwieweit und auf welche Weise die antizipierte Nachfrageentwicklung die einzelwirtschaftlichen Entscheidungsmuster bezüglich der Forschungspolitik beeinflusst. Zum anderen sind Versuche unternommen worden, die weitergehende Hypothese zu überprüfen, nach der die stärkeren Forschungsimpulse von der Nachfrage ausgehen und nicht von dem allgemeinen Fortschritt in der industriespezifischen Technologie, d.h. von den in den jeweiligen Industriezweigen vorherrschenden „technologischen Möglichkeiten“. ${ }^{202}$

Die frühe empirische Evidenz zur Schmookler-Hypothese wurde von Mowery und Rosenberg (1979) eingehend übergeprüft. Die kritische Auseinandersetzung der Autoren insbesondere mit der Methodik der frühen Studien führte zu der Feststellung, dass "the primacy of market demand forces within the innovation process is simply not demonstrated". ${ }^{203}$ Auch die Untersuchung von Kleinknecht und Verspagen (1988) kam zu dem Ergebnis, dass die Beziehung zwischen Nachfrage und Forschungstätigkeit schwächer ausfällt, als das

199 Vgl. Schmookler (1966), insbesondere S. 179-188.

200 Vgl. Schmookler (1966), S. $181 \mathrm{f}$.

201 Vgl. Hipp (2000), S. 28.

202 Vgl. Cohen (1995), S. 211.

${ }^{203}$ Mowery/Rosenberg (1979), S. 139. 
von Schmookler postuliert wurde. ${ }^{204}$ Eine neuere Studie von Geroski und Walters (1995), die die Granger-Kausalität zwischen Nachfrageveränderungen und Inventions- und Innovationstätigkeit untersuchte, hat gezeigt, dass die Kausalitätsbeziehung zwar von der Nachfrage zu der Innovationstätigkeit verläuft, aber nur eine begrenzte Rolle als Innovationsanreiz spielt. ${ }^{205}$

Im Gegensatz zu den frühen Untersuchungen zur Bedeutung der Nachfrage als einem der Innovationsanreize, die primär outputorientierte Indikatoren der Forschungstätigkeit verwendeten, können in der neueren Literatur mehrere Studien identifiziert werden, die inputorientierte Messgrößen einsetzen. Insbesondere liefern diejenigen Untersuchungen wichtige Aufschlüsse, welche die im Zuge der europaweiten Innovationserhebungen (CIS) gewonnen Unternehmensdaten auswerten. Einen positiven Einfluss der Nachfrage auf die Entscheidung der Unternehmen für die Aufnahme bzw. Ausweitung der FuETätigkeit bestätigen Untersuchungen, die auf Daten zur Forschungstätigkeit französischer ${ }^{206}$, niederländischer ${ }^{207}$ und Schweizer ${ }^{208}$ Unternehmen basieren. Dieses robuste Ergebnis wird zudem trotz weitgehender messtechnischer Unterschiede erzielt. So verwendet die niederländische Studie von Brouwer und Kleinknecht (1999b) die Umsatzwachstumsraten der jeweiligen Unternehmen (metrisch skalierte Variable), die Schweizer Studien die in der Erhebung abgefragten mittelfristigen Nachfrageerwartungen (ordinal skalierte Variable) und die französische Untersuchung von Crepon et al. (1996) einen auf Branchenebene aggregierten Indikator (demand-pull proxy ${ }^{209}$ ).

Drei spezielle Ergebnisse der Schweizer Studien sollen an dieser Stelle zusätzlich angesprochen werden. ${ }^{210}$ Erstens zeigt sich in diesen Untersuchungen, dass die Nachfrageerwartungen ausschlaggebend für die Grundlageentscheidung für oder gegen eine Aufnahme der Forschungstätigkeit zu sein scheinen und nicht so sehr für das Ausmaß der Forschungstätigkeit. ${ }^{211}$ Mit anderen Worten: Die

\footnotetext{
204 Vgl. Kleinknecht/Verspagen (1988), S. 16.

$205 \mathrm{Vgl}$. Geroski/Walters (1995), S. 926.

206 Vgl. Crepon/Duguet/Kabla (1996).

207 Vgl. Brouwer/Kleinknecht (1999b).

$208 \mathrm{Vgl}$. Arvanitis/von Arx (2004), Arvanitis (1997). In den Studien von Arvanitis und Hollenstein $(1994 a, 1996)$ sowie Lenz (1998) werden neben signifikant positiven auch vereinzelt insignifikante Ergebnisse bei geänderter Modellspezifikation erhalten.

209 Vgl. Crepon/Duguet/Kabla (1996), S. 68.

210 Zur Beschreibung des Schweizer Firmenpanels von KOF/ETH vgl. Arvanitis/Hollenstein (2004).

211 In der Studie von Lenz (1998) ist der Koeffizient bei der Variable „mittelfristige Nach-
} 
positiven Nachfrageerwartungen stellen lediglich eine notwendige Bedingung bei der Entscheidung für die Aufnahme der Forschungstätigkeit dar; ihr Einfluss auf die Forschungsintensität der Unternehmen fällt dagegen gering aus. Zweitens liefert die Studie von Arvanitis und Hollenstein (1996) einen Hinweis darauf, dass der Einfluss der mittelfristigen Nachfrageerwartung unterschiedlich stark für die Unternehmen verschiedener Größenklassen ausfällt. Für Klein- und Mittelunternehmen (unter 200 Beschäftigten) hat die antizipierte positive Nachfrageentwicklung stärkere Bedeutung als für Großunternehmen. ${ }^{212}$ Drittens wird in den Studien konstatiert, dass die Nachfragebedingungen einen schwächeren Einfluss auf die Forschungsaufwendungen der Unternehmen ausüben, als die in dem unternehmensexternen Umfeld vorherrschenden technologischen Möglichkeiten. ${ }^{213}$

\subsubsection{Aneignungsbedingungen}

Der Hinweis auf die Bedeutung von Aneignungsmöglichkeiten erscheint bereits implizit bei Schumpeter ${ }^{214}$, die explizite Analyse geht jedoch auf Arrow (1962) zurück. Die Argumentation hinter der Hypothese, Aneignungsbedingungen beeinflussten die Forschungstätigkeit im Wirtschaftssektor, kann folgendermaßen zusammengefasst werden: Um Forschungsaktivitäten zu betreiben, müssen privatwirtschaftliche Unternehmen in der Lage sein, von den Erträgen eigener Forschung zu profitieren. Da jedoch Forschungsergebnisse diffundieren und folglich der volkswirtschaftlich entstehende Nutzen aus dem neuerarbeiteten Wissen nicht vollständig von dem jeweiligen Produzenten angeeignet werden kann, sinkt der Anreiz eines einzelnen gewinngesteuerten Akteurs, in FuE zu

frageerwartungen" signifikant positiv, wenn sowohl Innovatoren als auch Nichtinnovatoren berücksichtigt werden. Bei ausschließlicher Berücksichtigung der Innovatoren verliert jedoch die mittelfristige Nachfrageerwartung an Einfluss, vgl. Lenz (1998), S. $134 \mathrm{ff}$., sowie auch die ähnlichen Ergebnisse in Arvanitis/von Arx (2004), S. 17.

${ }^{212} \mathrm{Zu}$ beachten ist allerdings, dass dieses Ergebnis bei Verwendung von einem Indikator (INNIN) zustande kommt, der neben FuE-Tätigkeit auch die Konstruktionstätigkeit einschließt, was ein breiteres Verständnis der Forschungstätigkeit impliziert, als die in der vorliegenden Arbeit vorgenommene Abgrenzung. Zur Beschreibung des Indikators vgl. Arvanitis/Hollenstein (1996), S. $18 \mathrm{ff}$; für die Schätzergebnisse vgl. S. $48 \mathrm{f}$.

213 Vgl. Arvanitis (1997), S. 477 ff., Arvanitis/Hollenstein (1996), S. 56, einschränkend gilt auch in diesem Falle die Anmerkung der vorangegangenen Fußnote.

214 Vgl. dazu die Anmerkung von Arrow (1962), S. 622: „The only ground for arguing that monopoly may create superior incentives to invent is that appropriability may be greater under monopoly than under competition." 
investieren. ${ }^{215}$ Damit bestimmt das Ausmaß der unfreiwilligen Wissensdiffusion (d.h. der positiven Externalitäten) die effektive Forschungsintensität der Unternehmen.

Die Anerkennung der eingeschränkten Aneignungsmöglichkeiten dient als eine der Begründungen für die Einführung der künstlich geschaffenen Schutzmechanismen in Form von Patenten. ${ }^{216}$ Mit der Gewährung von exklusiven Rechten auf das privatwirtschaftlich erarbeitete Wissen werden alle anderen Wirtschaftssubjekte von der kostenfreien Nutzung der Forschungsergebnisse ausgeschlossen oder müssen ein Entgelt (Lizenzgebühren) dem Wissensproduzenten entrichten. Damit wird das Problem der unfreiwilligen Wissensdiffusion neutralisiert; der Wissensproduzent erhält exklusive Nutzungsrechte und kann die für die Finanzierung der Forschungsvorhaben benötigten Monopolrenten erwirtschaften.

Der Diskussion der Problematik der Aneignungsbedingungen und der Begründung für die Etablierung der künstlichen Appropriierungstitel in Form von Patenten liegt die Konzeptualisierung vom Wissen als einer frei zugänglichen, sich rasch verbreitenden Information zugrunde. ${ }^{217}$ Nur wenn man voraussetzt, dass jedes Unternehmen in der Lage ist, die extern erarbeiteten Forschungserträge zu vernachlässigbaren Kosten zu übernehmen, intern umzusetzen und zu verwerten, stimmt die Schußfolgerung, dass Forschungsergebnisse unzureichend angeeignet werden können. Damit wird jedoch auch davon ausgegangen, dass FuE-betreibende Unternehmen begrenzte Möglichkeiten haben, eigene Mechanismen zum Schutz des erarbeiteten Wissens vor unfreiwilliger Diffusion zu entwickeln. Um diese Annahmen einer kritischen Prüfung zu unterziehen, wird im folgenden Abschnitt auf die Ergebnisse der empirischen Studien eingegangen, die die Wirksamkeit alternativer Schutzmechanismen untersuchen.

215 In dem Sinne definiert Audretsch das Aneignungsproblem als „the ability of firms to reap the economic returns accruing from their investment in knowledge-generating activities, such as R\&D“, Audretsch (1995), S. 39.

${ }^{216}$ Im Allgemeinen werden in der Literatur drei Gründe für die Gewährung exklusiver Patentrechte genannt: (1) Stimulierung von Erfindungen durch Verbesserung der Aneignungsbedingungen, (2) Förderung der Weiterentwicklung und kommerziellen Nutzung der Inventionen und (3) Erleichterung eines öffentlichen Zugangs zu Erfindungen, d.h. Förderung der Diffusion neuen Wissens, vgl. Audretsch (1995), S. 41.

217 Für die Konzeptionen von Wissen vgl. Abschnitt 2.3 ab der S. 44. 


\subsubsection{Studien zu alternativen Schutzmechanismen}

Als klassische Untersuchung zu unternehmensinternen Appropriierungsmechanismen gilt die Studie von Levin et al. (1987). Die Autoren gingen in der Studie von der Beobachtung aus, dass das Patentsystem in der Praxis nur eine unzureichende Wirkung als Schutzmechanismus entfaltet ${ }^{218}$ und oft durch andere, durch die Unternehmen geschaffene Schutzmechanismen ersetzt wird. Sie identifizierten insgesamt sechs verschiedene Aneignungsmethoden (methods of appropriation) und setzten sich zum Ziel, sie einer vergleichenden Analyse zu unterziehen. $\mathrm{Zu}$ den untersuchten Instrumenten zum Schutz der aufgrund von Inventionen entstandenen Wettbewerbsvorteile gehörten (1) Patente zum Schutz vor Imitation, (2) Patente, um Lizenzzahlungen Dritter zu sichern, (3) Geheimhaltung, (4) Vorlaufzeit (lead time), (5) Realisierung von Lernkurveneffekten und (6) Verkaufs- und Servicemaßnahmen.

Insgesamt 650 Vertreter von FuE-aktiven Unternehmen wurden in einer Umfrage gebeten, die relative Wirksamkeit einzelner Schutzmechanismen auf einer siebenstufigen Likert-Skala (1 - nicht effektiv bis 7 - sehr effektiv) einzuschätzen. Die Befragung lieferte folgende Ergebnisse. Erstens bestätigte sich die Hypothese der eingeschränkten Wirksamkeit der alternativen Schutzmechanismen: Durchschnittswerte der Wirksamkeit aller von den Autoren vorgeschlagenen Schutzmaßnahmen lagen unter dem Wert von 5.6. ${ }^{219}$ Insofern als einzelwirtschaftliche Angaben zur Wirksamkeit der Schutzmechanismen als Indikator des Grades der Aneignungsmöglichkeiten des innovationsrelevanten Wissens aufgefasst werden können, bestätigte diese Studie folglich das Vorliegen der positiven Externalitäten. Inwieweit sich ein signifikanter (negativer) Einfluss der festgestellten unvollständigen Appropriierbarkeit der Forschungsergebnisse auf die Entscheidungen der Unternehmen im FuE-Bereich feststellen lässt, wird Gegenstand der Ausführungen im nächsten Abschnitt sein.

Zweitens erwies sich die Wirksamkeit der Patente als deutlich unterlegen gegenüber marktstrategischen Instrumenten wie zeitlicher Vorsprung, Verkaufsund Servicemaßnahmen sowie Realisierung von Lernkurveeffekten. ${ }^{220} \mathrm{Im}$ Falle der Prozesstechnologien wurde auch die Geheimhaltung als wirksamer als der Patentschutz eingestuft. Folglich zeigte die Studie, dass Unternehmen in der Lage sind, eigene Instrumente zum Schutz innovationsrelevanten Wissens

${ }^{218}$ Für die Gründe des „Versagens" des Patentsystems vgl. Levin/Klevorick/Nelson/Winter (1987), S. 784 sowie die dort zitierte Literatur.

219 Vgl. Levin/Klevorick/Nelson/Winter (1987), S. 794.

$220 \mathrm{Vgl}$. Levin/Klevorick/Nelson/Winter (1987), S. 794. 
vor unfreiwilliger Diffusion zu entwickeln, die hinsichtlich der beabsichtigten Wirkung effektiver sind als staatlich geschaffene Aneignungstitel (Patente).

Drittens wurden erhebliche interindustrielle Unterschiede festgestellt. ${ }^{221} \mathrm{~Pa}$ tente schienen eine besondere Wirkung in der chemischen Industrie (Pharma, organische und anorganische Chemie) zu entfalten. Nur in der Pharmaindustrie wurde der Patentschutz als der wirksamste Schutzmechanismus eingestuft. Die besonders hohe Einschätzung der Wirksamkeit des Patentschutzes in der chemischen Industrie wurde von den Autoren damit begründet, dass im Falle der chemischen Produkte aufgrund der nahezu vollkommenen Kodifizierbarkeit des gewonnenen Wissens sowohl die Patentanmeldung als auch eine Verteidigung gegen Patentverletzungen relativ einfach zu bewerkstelligen sind. ${ }^{222}$ Insofern wurde ein Hinweis auf die Notwendigkeit einer Unterscheidung geliefert, zwischen Technologiefeldern, in denen der Output der Forschungsanstrengungen die Eigenschaften eines öffentlichen Gutes aufgrund guter Kodifizierbarkeit aufweist und folglich schwache Appropriierungsmöglichkeiten jenseits des Patentwesens vorliegen, und Technologiefeldern mit einem größeren Anteil der impliziten Komponenten des Wissens, für die strategische Aneignungsmethoden eine größere Rolle spielen können.

In den vergangenen zehn Jahren hat eine Reihe von Untersuchungen den von Levin et al. (1987) etablierten Untersuchungsansatz verfolgt, um deren Ergebnisse mit umfangreicheren Datensätzen und Daten von nicht USamerikanischen Unternehmen zu verifizieren. ${ }^{223}$ Die oben erläuterten Befunde von Levin et al. sind dabei weitestgehend reproduziert worden. Auch bei Änderung bzw. Ergänzung der Liste der Schutzmechanismen erwiesen sich die durch den Staat gewährten, rechtlichen Aneignungstitel (Patente und Gebrauchsmuster) als eindeutig unterlegen gegenüber ausgewählten marktstrategischen Instrumenten. ${ }^{224}$

Angesichts der Tatsache, dass Patente und Gebrauchsmuster insgesamt als relativ unwirksam eingestuft wurden, stellte sich die Frage nach der Begründung für die intensive Inanspruchnahme der rechtlichen Schutzmeclianismen. Zwei verschiedenartige Erklärungsstränge können in der Literatur identifiziert werden.

221 Vgl. Levin/Klevorick/Nelson/Winter (1987), S. 797.

222 Vgl. Levin/Klevorick/Nelson/Winter (1987), S. 798.

223 Vgl. Harabi (1995), König/Licht (1995), Arundel (2001), Sattler (2003), Cohen/Goto/Nagata/Nelson/Walsh (2002).

${ }^{224}$ Drei zusätzliche Schutzmaßnahmen sind insgesamt identifiziert worden: langfristige Bindung der Mitarbeiter, Gebrauchsmuster und Komplexität der Produktgestaltung. 
Zum einen wird die Hypothese untersucht, ob rechtliche Schutzmechanismen ein wichtiges Appropriierungsinstrument für bestimmte Subpopulationen der Unternehmen darstellen. Bereits in der Studie von Levin et al. (1987) ist angedeutet worden, dass Unternehmen der Pharmaindustrie eine solche Subgruppe bilden können. Sattler (2003) bestätigte in einer Regressionsanalyse, dass die Zugehörigkeit eines Unternehmens zur Chemiebranche einen hochsignifikanten Einfluss auf die Einschätzung der Wirksamkeit des Patenschutzes ausübt. ${ }^{225}$ Als zweite Subpopulation wurde die Gruppe der Klein- und Mittelunternehmen vermutet mit der Begründung, dass diese Unternehmen weniger in der Lage sind, eigene Instrumente zur Sicherung der Monopolrenten $\mathrm{zu}$ entwickeln. ${ }^{26}$ Für diese Hypothese wurde allerdings in der Untersuchung von Arundel (2001) keine Bestätigung gefunden; Sattler (2003) zeigte im Gegenteil, dass gerade Großunternehmen die Effektivität der Patente höher einschätzen. ${ }^{27}$ Interessante Hinweise auf eine weitere Subpopulation lieferte die Studie von Harabi (1995), in der der Autor neben sieben Industriebranchen zwei Dienstleistungsgruppen unterschied: technische Dienstleistungen und private Forschungslabors. Dabei zeigte sich, dass die privaten Forschungslabors die Wirksamkeit der Patente zur Sicherung von Lizenzzahlungen höher als alle sonstigen Gruppen einschätzten. ${ }^{228}$ Es kann folglich vermutet werden, dass das Patentwesen eine hohe Bedeutung für die Gruppe der Forschungsdienstleister hat.

Zum anderen ist die Hypothese formuliert worden, dass Patente nicht nur als ein Instrument zur Sicherung der Monopolrenten aufgefasst werden, sondern - zunehmend - marktstrategische Funktionen erfüllen: ${ }^{29}$

- aufgrund der Kumulativität des Wissens stellen Patente ein Instrument dar, mit dem die Forschungsprojekte der Wettbewerber in gleichen oder angrenzenden Gebieten erschwert bzw. blockiert werden können (die sog. offensive Blockade),

- spiegelbildlich werden die Patente angemeldet, um zu verhindern, dass der eigene technologische Spielraum durch Patente anderer vermindert wird (die sog. defensive Blockade)

225 Vgl. Sattler (2003), S. 512.

226 Vgl. Arundel (2001), S. 611 ff., Levin/Klevorick/Nelson/Winter (1987), S. 797.

227 Vgl. Sattler (2003), S. 512.

228 Vgl. Harabi (1995), S. 986.

229 Vgl. Arora (1997), Reitzig (2004), Shapiro (2001), Blind/Edler/Frietsch/Schmoch (2003), S. 16 ff. sowie die dort zitierte Literatur. 
- Patentportfolios können als Eintrittsbarriere fungieren, wenn Markteinsteiger auf patentierte Lösungen der Wettbewerber des relevanten Marktes angewiesen sind,

- eigene Patente können ferner als ein Instrument zur Verbesserung der eigenen technologischen Reputation verwendet werden, was zur Stärkung der Verhandlungsposition des Unternehmens gegenüber

- anderen Unternehmen (bei Zusammenschluss und Übernahmeverhandlungen oder Kreuzlizenzierungen),

- Kreditgebern

- dem Staat (in Belangen der Technologiepolitik) oder

- dem Ausland (beim internationalen Markteintritt)

relevant sein kann. ${ }^{230}$

\subsubsection{Empirische Evidenz}

Die theoretischen Überlegungen zur Bedeutung der Aneignungsbedingungen für die Investitionsentscheidungen in FuE sowie die wiederholt bestätigten Befunde von Levin et al. (1986) zur eingeschränkten Wirksamkeit von Schutzmechanismen fanden auch ihren Niederschlag in der Konzeption der empirischen Untersuchungen zu den Determinanten der Forschungstätigkeit im Wirtschaftssektor. Werden die einzelnen Schutzmechanismen aus einzelwirtschaftlicher Perspektive als unwirksam eingestuft, so bedeutet das, dass eine unvollständige Appropriierungsmöglichkeit vorliegt und die betroffenen Unternehmen die Stellung eines Emittenten technologischer Spillovers einnehmen. Folglich wurden in den neueren Untersuchungen zu den Bestimmungsgründen

${ }^{230}$ Auf die zunehmende Bedeutung der strategischen Nutzung des Patentsystems als eine Erklärung für den seit 1995 beobachtbaren starken Anstieg der Zahl der Patentanmeldungen verweisen Autoren des Frauenhofer ISI in ihrer Studie zum Patentverhalten deutscher Unternehmen. Als „strategische Motive der Patentierung“ definieren die Autoren „alle Motive, welche über den Schutz eigener Erfindungen zur Aneignung des auf dieser Erfindung beruhenden Nutzens auf den relevanten Märkten hinausgehen“ (S. 2.). Die patentstatistische Analyse sowie eine Befragung von über 500 Unternehmen mit einer Mindestanzahl von Patenten führt zu der Schlussfolgerung, dass der Anstieg der Patenanmeldungen nicht auf die stark gestiegene Forschungseffizienz zurückzuführen ist (d.h. verbessertes Verhältnis zwischen Forschungsinput und -output), sondern maßgeblich durch die insgesamt stark an Bedeutung gewinnende strategische Motivation der Patentierung in der Subpopulation der Großunternehmen bestimmt wird, vgl. Blind/Edler/Frietsch/Schmoch (2003), S. XII f. 
der FuE-Tätigkeit die in Anlehnung an die Methodologie von Levin et al. (1987) erhobenen Daten zur Wirksamkeit bzw. Bedeutung der Schutzmaßnahmen einbezogen und als Proxy für den Grad der Appropriierbarkeit der Forschungsergebnisse verwendet.

Bei der Analyse der Ergebnisse der Untersuchungen, die die subjektive Einschätzung der Wirksamkeit alternativer Schutzmechanismen als Proxy für den Grad der Appropriierbarkeit verwenden, gilt es Unterschiede in der Methodologie zu beachten, da die auf Unternehmensebene erhobenen Angaben faktoranalytisch zu wenigeren Variablen verdichtet werden.

In den Schweizer Studien von Arvanitis und Hollenstein (1996) sowie Lenz (1998) werden Daten zur Wirksamkeit von sieben verschiedenen Schutzmechanismen faktoranalytisch zu drei Variablen verdichtet. Dabei repräsentiert die erste Variable die rechtlichen Schutzmechanismen (Patente und Gebrauchsmuster), die zweite Variable - die drei marktstrategischen Schutzmechanismen (Geheimhaltung, Komplexität der Produktgestaltung und Zeitvorsprung) und die dritte Variable - zwei weitere informale Schutzmechanismen (langfristige Bindung spezialisierten Personals und überragende Verkaufs- und Serviceleistungen). ${ }^{231}$ Unabhängig von den verwendeten Indikatoren der Forschungstätigkeit zeigt sich ein signifikant positiver Einfluss der Variable, die die drei marktstrategischen Schutzmechanismen (Geheimhaltung, Komplexität der Produktgestaltung und Zeitvorsprung) abbildet. Kein signifikanter Einfluss der Wirksamkeit von rechtlichen Schutzmechanismen (Patente und Gebrauchsmuster) ist feststellbar bei Berücksichtigung von Innovatoren und Nichtinnovatoren. Werden in den Regressionsgleichungen auschließlich innovierende Unternehmen berücksichtigt, dann zeigt sich vereinzelt in der Studie von Lenz (1998) auch ein positiver Einfluss der Wirksamkeit rechtlicher Schutzmechanismen. Der Einfluss der dritten Variable, die informale Schutzmaßnahmen wie langfristige Bindung der Mitarbeiter bzw. überragende Verkaufs- und Servicemaßnahmen repräsentiert, erweist sich vorwiegend als nicht signifikant. ${ }^{232}$

Becker und Dietz (2002) verwenden die in der ersten Welle des Mannheimer Innovationspanels (MIP 1993) erhobenen Daten zur Bedeutung von sechs Maßnahmen zum Schutz innovationsrelevanten Wissens im Bereich der Prozessund Produkttechnologien und extrahieren zwei Faktoren: Der erste repräsentiert rechtliche, der zweite strategische Schutzmaßnahmen. ${ }^{233}$ In simultanen

${ }^{231}$ Für die Faktorladungen vgl. Arvanitis/Hollenstein (1996), S. 24, Lenz (1998), S. 126.

232 Vgl. Arvanitis/Hollenstein (1996), S. 30, Lenz (1998), S. 134 ff.

${ }^{233}$ Für die Faktorladungen vgl. Becker/Dietz (2002), S. 28. 
Schätzungen der FuE-Intensität und Kooperationstätigkeit zeigt sich ein positiver Einfluss der rechtlichen Schutzmaßnahmen auf die FuE-Intensität; in zwei von vier Gleichungen ist der Einfluss der strategischen Schutzmechanismen signifikant positiv. ${ }^{234}$ In der Studie von Becker und Peters (2005) wird ebenfalls der Einfluss von rechtlichen und strategischen Appropriierungsmaßnahmen untersucht: beide Faktoren beeinflussen in positiver Weise die Entscheidung für FuE-Tätigkeit. Ein signifikant positiver Einfluss beider Faktoren auf die Forschungsintensität der Unternehmen zeigt sich auch, wenn die FuE-Intensität durch den (logarithmierten) Anteil des FuE-Personals an der Gesamtzahl der Beschäftigten abgebildet wird. ${ }^{235}$

In der Literatur können auch andere Ansätze zur Operationalisierung des Konzepts der Aneignungsbedingungen vorgefunden werden. Arvanitis und Hollenstein (1994a, 1996) verwenden eine Variable, die auf globalen Einschätzungen der Unternehmen zur Wirksamkeit des Schutzes innovationsbedingter Wettbewerbsvorteile beruht und schätzen ihren Einfluss auf die Forschungsintensität der Unternehmen. Dabei zeigt sich in der ersten der beiden Studien, dass sich eine höhere Einschätzung der Wirksamkeit der Schutzmechanismen hochsignifikant positiv auf die Forschungsintensität der Unternehmen auswirkt; in der zweiten Studie ist jedoch kein signifikanter Einfluss feststellbar. ${ }^{236}$ Die gleiche Variable wird in eine ebenfalls auf die Daten des Schweizer Innovationspanels zurückgreifende Studie von Arvanitis und von Arx (2004) einbezogen. Trotz der Unterschiede in den verwendeten Schätzverfahren ${ }^{237}$ bestätigt sich ein signifikant positiver Einfluss der effektiveren Appropriierung auf die Aufnahme bzw. Ausweitung der FuE-Tätigkeit. In der Studie von Felder et al. (1996) wird eine Dummy-Variable verwendet, die auf der Angabe der Unternehmen basiert, inwieweit sie eine niedrige Rentabilität aufgrund von Imitationsgefahr antizipieren. Der Einfluss dieser Variable auf die Forschungsintensität der deutschen Unternehmen erweist sich je nach abhängiger Variable als nicht signifikant bzw. - erwartungsgemäß - signifikant negativ. ${ }^{238}$

234 Vgl. Becker/Dietz (2002), S. 26.

235 Vgl. Becker/Peters (2005), S. 141.

$236 \mathrm{Vgl}$. Arvanitis/Hollenstein (1994a), S. 25 und 27, Arvanitis/Hollenstein (1996), S. 32.

237 Im Unterschied zu Arvanitis und Hollenstein (1994a) werden in dieser Studie keine Querschnitts- sondern mehrere Längsschnittauswertungen vorgenommen („gepoolte" Logit-Schätzungen und Random-Effekt-Modelle), zur tabellarischen Zusammenstellung der abhängigen Variablen und verwendeten Schätzverfahren vgl. Arvanitis/von Arx (2004), S. 39.

238 Vgl. Felder/Licht/Nerlinger/Stahl (1996), S. $141 \mathrm{ff}$. 


\subsubsection{Technologische Möglichkeiten}

Hinter dem Konzept der technologischen Möglichkeiten verbirgt sich die Beobachtung, dass die technologischen Rahmenbedingungen in ausgewählten Branchen innovationsfreudiger sind als in den anderen. ${ }^{239}$ So wird in ausgewählten Wirtschaftszweigen öffentliche Grundlagenforschung betrieben, von deren Ergebnissen die angewandte Forschung im Wirtschaftssektor profitieren kann. In den öffentlichen Forschungseinrichtungen werden auch qualifizierte Fachkräfte ausgebildet, auf deren Qualifikation die private Wirtschaft zurückgreifen kann. In bestimmten Branchen wird zudem kontinuierlich mehr FuE betrieben, so dass einzelne Unternehmen dieser Branchen auf eine breitere Wissensbasis und unternehmensextern erarbeitete Erfahrung (sog. accumulated stock of knowledge) zurückgreifen können. Diese aus der Sicht eines Unternehmens exogen gegebenen Aspekte beeinflussen seine privatwirtschaftliche FuE-Kostenfunktion: Je günstiger die Konstellation der extern zugänglichen Wissensressourcen, desto geringer ist der nötige Forschungsaufwand einer einzelnen Unternehmung, um eine wirtschaftlich verwertbare Invention hervorzubringen. ${ }^{240}$ Die hohe Ertragsrate soll folglich die Ausweitung der industriellen FuE begünstigen. Bei eingeschränkter externer Wissensbasis dagegen, d.h. bei geringeren technologischen Möglichkeiten, ermöglichen erst umfangreiche Investitionen die Erforschung der potenziellen Neuerungen. Da jedoch die Erfolgsaussichten aufgrund der schwächeren Ausgangsbasis - in der Verbindung mit der stochastischen Natur des Forschungsvorganges - geringer ausfallen, kann von schwächeren Innovationsanreizen der Unternehmen ausgegangen werden, die in einem durch geringe technologische Möglichkeiten gekennzeichneten Umfeld agieren. ${ }^{241}$

Im Rahmen der empirischen Forschung sind zahlreiche Vorschläge zur Operationalisierung des Konzepts der technologischen Möglichkeiten entwickelt worden. Frühe Ansätze versuchten, die Variation der technologischen Möglichkeiten auf der Branchenebene zu erfassen. So wurden in den älteren Studien verschiedene Taxonomien der Industriebranchen entwickelt. ${ }^{242}$ Dabei ist vorausgesetzt worden, dass zwischen ausgewählten Branchen so weit gehende Ähnlichkeiten bestehen, dass eine Einteilung in einige wenige Sektoren bereits

239 Vgl. Gottschalk/Janz (2003), S. 30, Cohen (1995), S. 214.

240 Daher wurden die „technologischen Möglichkeiten“ in der Literatur auch als intersektorale Variation der Kosten des einzelwirtschaftlichen FuE-Produktionsvorgangs definiert, Vgl. Jaffe (1986), S. 984.

241 Vgl. Meschede (2003), S. 44.

242 Vgl. z.B. Scherer (1965), S. 1107, Jaffe (1986), S. 985 ff. 
den größten Teil der Varianz der intersektoralen Unterschiede in den technologischen Rahmenbedingungen aufgreifen könnte.Alternativ sind auch einfache Branchen-Dummys in Regressionsgleichungen als Kontrollvariablen eingesetzt worden. ${ }^{243}$ Eine weitere Proxy-Variable bildete das Alter der Industrie, da die Lebenszyklusmodelle der Technologien die Ausschöpfung von technologischen Möglichkeiten in den späten Phasen postulieren. ${ }^{244}$ Ferner wurden auch Variablen eingesetzt, die die gesamte Forschungstätigkeit der Unternehmen einer Industrie aggregieren und als Proxy für den „Spillover-Pool“ verwenden. ${ }^{245}$

Neuere Studien versuchen dagegen verstärkt, die Variation der technologischen Möglichkeiten auf der Mikroebene zu erfassen. So verwenden einige Studien rangskalierte subjektive Einschätzungen der einzelnen Unternehmen über das in ihrem Umfeld vorherrschende technologische Potenzial. ${ }^{246}$ In mehreren Untersuchungen werden die technologischen Möglichkeiten durch Variablen abgebildet, die die Bedeutung der externen Wissensquellen für die unternehmerische Forschungstätigkeit erfassen. ${ }^{247}$ Dabei wird die Tatsache, dass Unternehmen auf externe Wissensquellen zurückgreifen, als ein Hinweis darauf interpretiert, dass in dem unternehmensexternen Umfeld das für eine bestimmte Invention benötigte (Input-) Wissen bereits erarbeitet worden ist. ${ }^{248}$

Nachfolgend sollen zunächst in Abschnitt 3.4.3.1 konzeptionelle Grundlagen der Erfassung von technologischen Möglichkeiten auf Branchenebene exemplarisch anhand der Typologie von Pavitt erläutert werden. Die bestehende empirische Evidenz zum Einfluss der auf der Mikroebene erfassten technologischen Möglichkeiten auf die Entscheidungen der Unternehmen im FuE-Bereich werden in Abschnitt 3.4.3.2 zusammengestellt.

\subsubsection{Die Typologie von Pavitt}

Stellvertretend für das auf Branchenebene erfasste Konzept der technologischen Möglichkeiten wird die Taxonomie von Pavitt (1984) näher besprochen,

\footnotetext{
$243 \mathrm{Vgl}$. z.B. für eine neuere Untersuchung Gottschalk/Janz (2001), S. 8.

$244 \mathrm{Vgl}$. Levin/Reiss (1984), S. 186, Levin/Cohen/Mowery (1985), S. 22.

245 Vgl. Licht/Zoz (2000), S. 318.

246 Vgl. die Studien von Arvanitis (1997), Arvanitis/von Axt (2004), Lenz (1998).

247 Vgl. Felder/Licht/Nerlinger/Stahl (1996), Becker/Dietz (2002), Arvanitis (1997), Arvanitis/von Axt (2004).

${ }^{248}$ Mehrere potentielle unternehmensexterne Quellen sind dabei unterschieden worden; zu den meist untersuchten gehörten Kunden, Zulieferer und öffentliche Forschungseinrichtungen.
} 
da sie zum einen - ähnlich wie die mikroökonomisch geprägte Operationalisierungsansätze - auf der Bedeutung der inter- und intrasektoralen Verflechtungen in der Wissensproduktion basiert und zum anderen auch in den neueren Untersuchungen vereinzelt Verwendung findet. ${ }^{249}$

Pavitt ging in seiner Studie von der deskriptiven Analyse ausgewählter britischer Wirtschaftszweige aus und beobachtete dabei erhebliche intersektorale Unterschiede in der Bedeutung der einzelnen Wissensquellen (der unternehmensinternen FuE, der Forschungstätigkeit anderer Unternehmen bzw. der öffentlichen Einrichtungen), der relativen Anteile der Prozess- und Produktinnovationen sowie der verwendeten Aneignungsstrategie. Auf dieser Grundlage schlug er eine Typologie der Technologiefelder vor, die auf einer Zuordnung der einzelnen Branchen in drei (bzw. vier, bei weiterer Unterteilung des produktionsintensiven Sektors) verschiedene Sektoren basiert: ${ }^{250}$

- Der wissensbasierte Sektor umfasst die chemische und elektronische Industrie. Die Firmen dieser Branchen basieren weitgehend auf firmeninterner FuE, allerdings unterhalten sie enge Kontakte zu öffentlichen Einrichtungen, da sie auf den Ergebnissen der öffentlichen Grundlagenforschung in ihrer eigenen Innovationstätigkeit aufbauen. Die Unternehmen dieser Branchen sind in der Vergangenheit auf eine dynamische Nachfrage gestoßen, was erfolgreichen Unternehmen zu erheblichen Wachstumsraten verholfen und die Anreize zur vertikalen Diversifizierung jenseits der Kernaktivitäten geschwächt hat. Unternehmen des wissensbasierten Sektors nutzen einen Mix an Aneignungsstrategien; für Feinchemikalien spielt jedoch der Patentschutz eine herausragende Rolle. Lernkurveneffekte haben sich zugleich im Laufe der Zeit als wichtige Marktzutrittsbarriere erwiesen.

- Der lieferantenbeherrschte Sektor besteht aus relativ kleinen Unternehmen der durch eine niedrige Innovationsrate gekennzeichneten Branchen (Textilindustrie, Bauwesen). Die Innovationen der Unternehmen dieses Sektors zielen meist auf Kostenreduktion ab, da die Firmen dieser Branchen auf eine hohe Preiselastizität der Nachfrage treffen. Allerdings werden die Prozessinnovationen nur zum kleinen Teil durch interne Forschungsanstrengungen erarbeitet; die technologische Basis dieser Unter-

249 Vgl. z.B. Vossen (1999), Vossen/Nooteboom (1996), Kleinknecht/Oostendorp (2002), Brouwer/Kleinknecht (1999b).

250 Vgl. Pavitt (1984), S. 353 ff. 
nehmen ist schwach, so dass die eingesetzten Innovationen oft aus anderen Industriezweigen kommen.

- Der produktionsintensive Sektor umfasst skalenintensive, große, diversifizierte Unternehmen der Lebensmittel-, Metall-, Schiffsbau- und Automobilindustrie sowie kleine Spezialanbieter aus der Maschinenbau- und Instrumentensparte. Skalenintensive Unternehmen greifen oft auf das extern erarbeitete Wissen zurück und stützen ihren komparativen Vorteil auf die Forschungsanstrengungen der Zulieferer. Innovationen werden durch Patente bzw. einen zeitlichen Vorsprung in der Markteinführung geschützt. Spezialanbieter spezialisieren sich auf die auf Bedürfnisse der Kunden zugeschnittene Produktinnovationen. Eigene Prozesstechnologien schützen sie meist durch die Geheimhaltung, bei eigenen Produktinnovationen setzten sie auf Lernkurveneffekte und unternehmensinterne Erfahrung.

\subsubsection{Empirische Evidenz}

Verwendet man die subjektiven, auf der Mikroebene erfassten, allgemeinen Einschätzungen der einzelnen Unternehmen über die relative Vorteilhaftigkeit der in ihrem Umfeld vorherrschenden technologischen Möglichkeiten, erweisen sich die Ergebnisse als relativ robust. In mehreren Studien, die auf den Daten des Schweizer Innovationspanels basieren, zeigt sich ein signifikant positiver Einfluss des technologischen Potenzials auf die Forschungsintensität der Unternehmen. ${ }^{251} \mathrm{Zu}$ sehr unterschiedlichen Ergebnissen führen dagegen die Untersuchungen, die ebenfalls auf Unternehmensebene erhobene Angaben zur relativen Bedeutung der externen Wissensquellen für die Innovationstätigkeit einzelner Unternehmen auswerten. Bevor jedoch auf die einzelnen Ergebnisse der Studien eingegangen wird, die diesen Indikator verwenden, soll nachfolgend kurz auf die Besonderheiten dieser Konzeptualisierung von technologischen Möglichkeiten eingegangen werden.

Die Operationalisierung des Konzepts der technologischen Möglichkeiten unter Zuhilfenahme von Variablen, die den Rückgriff der Unternehmen auf extern zugängliche Wissensquellen abbildet, verdeutlicht nämlich seine Verwandtschaft mit dem Problem der positiven Externalitäten der Forschungstätigkeit

251 Vgl. Arvanitis/von Arx (2004), Arvanitis/Hollenstein (1996), S. 30 ff. In der Studie von Lenz werden in Abhängigkeit von der gewählten abhängigen Variable neben signifikant positiven auch insignifikante Ergebnisse erhalten, vgl. Lenz (1998), S. $134 \mathrm{ff}$. 
bzw. der Spillover-Problematik. ${ }^{252}$ Sind die externen Wissensquellen gewichtig für die eigene Forschungstätigkeit eines Unternehmens, so bedeutet das, dass dieses Unternehmen die Stellung eines Wissensempfängers annimmt. ${ }^{253}$ Insofern setzen die Studien zur Bedeutung von technologischen Möglichkeiten und zu Aneignungsbedingungen an dem gleichen Phänomen der Wissensexternalitäten an und betrachten es aus zwei verschiedenen Perspektiven. Während die Studien zur Aneignungsmöglichkeiten untersuchen, inwieweit besserer Schutz vor unfreiwilligen Spillovers die Forschungstätigkeit stimuliert, überprüfen die Studien zur Bedeutung der technologischen Möglichkeiten, inwieweit sich die Fähigkeit der Wirtschaftsakteure, Wissensexternalitäten für unternehmensinterne Zwecke zu internalisieren, positiv auf ihre Forschungstätigkeit auswirkt.

Die in Umfragen erhobenen Daten zur Bedeutung von externen Wissensquellen werden in den meisten empirischen Studien zu Determinanten der FuE-Tätigkeit - ähnlich wie die Daten zum Grad der Appropriierbarkeit der Forschungsergebnisse - faktoranalytisch zu wenigen Variablen verdichtet.

In der Untersuchung von Felder et al. (1996) werden zwei Faktoren extrahiert: der erste erfasst die Bedeutung der wissenschaftlichen Wissensquellen, der zweite die von nichtwissenschaftlichen Wissensquellen (d.h. Informationen von Zulieferern, Kunden und Wettbewerbern). ${ }^{254}$ Es zeigt sich ein signifikant positiver Einfluss der wissenschaftlichen und ein insignifikanter Einfluss der nichtwissenschaftlichen Wissensquellen sowohl auf die Forschungsneigung als auch auf die Forschungsintensität der Unternehmen. ${ }^{255}$ Die Ergebnisse der Faktorenanalyse von Felder et al. (1996) werden auch in der Studie von Licht und Zoz (2000) verwendet, in der Bestimmungsgründe der Patentaktivität deutscher Unternehmen untersucht werden. Auch in dieser Untersuchung wirkt sich nur der erste Faktor, der die Bedeutung der wissenschaftlichen Wissensquellen erfasst, signifikant positiv auf die Patentneigung und die Anzahl der Patentanträge der Unternehmen aus. ${ }^{256}$ Becker und Dietz (2002), die ebenfalls Daten des Mannheimer Innovationspanels zum Innovationsverhalten deutscher Unternehmen verwenden, extrahieren drei Faktoren: der erste Faktor repräsentiert die relative Bedeutung der wissenschaftlichen Wissensquellen, der zweite - die des Wissens von Zulieferern und der dritte - die der Informatio-

252 Vgl. Meschede (2003), S. 58.

253 Vgl. Lenz (1998), S. 105.

${ }^{254}$ Für die Faktorladungen vgl. Felder/Licht/Nerlinger/Stahl (1996), S. 150.

255 Vgl. Felder/Licht/Nerlinger/Stahl (1996), S. 141.

256 Vgl. Licht/Zoz (2000), S. 326. 
nen von Kunden und Wettbewerbern. ${ }^{257}$ In einer simultanen Schätzung der Forschungsintensität und der Kooperationstätigkeit zeigt sich, dass eine hohe Bedeutung der Informationen aus wissenschaftlichen Einrichtungen keinen signifikanten Einfluss auf die Forschungsintensität ausübt, während die von Kunden und Wettbewerbern die Forschungsintensität signifikant steigert. Eine hohe Einschätzung der Bedeutung von Zulieferern als einer Wissensquelle verringert dagegen signifikant die FuE-Intensität der Unternehmen. In einer Studie von Becker und Peters (2005) zeigt sich ein hochsignifikanter Einfluss von wissenschaftlichen Quellen sowohl auf die Forschungsneigung als auch auf die Forschungsintensität der Unternehmen. Der Einfluss des Faktors, der Wissen von Kunden und Wettbewerbern repräsentiert, zeigt sich nur vereinzelt signifikant. ${ }^{258}$

Zu einer Dreifaktoren-Lösung kommen auch Arvanitis und Hollenstein (1996). Der erste Faktor erfasst in dieser Studie wissenschaftliche Wissensquellen; der zweite beschreibt die Bedeutung von „zugekauftem Wissen“ (Lizenzen, Investitionsgütern, angeworbenen wissenschaftlichen Personals sowie übernommenen Unternehmen); der heterogene dritte Faktor beinhaltet Wissen von Kunden, Wettbewerbern, Zulieferern sowie Messen und Fachzeitschriften. ${ }^{259}$ In den Regressionsgleichungen zeigt sich jedoch kein signifikanter Einfluss dieser Faktoren auf die Forschungsintensität der Unternehmen; lediglich eine hohe Bedeutung der wissenschaftlichen Wissensquellen wirkt sich signifikant positiv auf die Forschungsintensität der Produktinnovatoren aus. ${ }^{260}$

In der auf Daten zur Forschungstätigkeit finnischer Unternehmen basierenden Untersuchung von Leiponen (2002) werden die Angaben zur Bedeutung von Wissensquellen nicht faktoranalytisch verdichtet, sondern gehen als BranchenDurchschnitte in die Probit-Schätzung der FuE-Tätigkeit ein. Eine hohe Bedeutung des Wissens von Universitäten und Kunden erhöht die Wahrscheinlichkeit für die Aufnahme der FuE-Tätigkeit; eine hohe Bedeutung des Wissens von Zulieferern und Wettbewerbern hat dagegen eine reduzierte Wahrscheinlichkeit der FuE-Tätigkeit zur Folge. ${ }^{261}$ In der Studie von Arvanitis und Hollenstein (1994a) gehen die einzelwirtschaftlichen Angaben zur Bedeutung von Wissensquellen direkt, d.h. auf der Unternehmensebene variierend, in die Regressionsgleichungen ein, Arvanitis und von Arx (2004) wandeln dagegen die

${ }^{257}$ Für die Faktorladungen vgl. Becker/Dietz (2002), S. 29.

258 Vgl. Becker/Peters (2005), S. 141.

259 Für die Faktorladungen vgl. Arvanitis/Hollenstein (1996), S. 26.

$260 \mathrm{Vgl}$. Arvanitis/Hollenstein (1996), S. 32 f.

261 Vgl. Leiponen (2002), S. 271. 
ordinalskalierte Variablen in binäre Variablen um. Eine hohe Einschätzung des Wissens von Wettbewerbern führt in der zweiten Studie insbesondere zur Reduzierung der Wahrscheinlichkeit der Aufnahme von FuE-Tätigkeit.

Insgesamt ergibt sich auf den ersten Blick ein uneindeutiges Bild. Eine genauere Analyse lässt jedoch folgendes Muster bezüglich der Bedeutung der technologischen Möglichkeiten für die Forschungstätigkeit im Wirtschaftssektor vermuten: Im Allgemeinen begünstigt das vorhandene technologische Potenzial die Entscheidung für die Aufnahme der Forschungstätigkeit. Bei der Untersuchung der Bedeutung der einzelnen externen Wissensquellen zeigt sich jedoch, dass das externe Wissen zum Teil substitutiv und zum Teil komplementär zu eigener FuE der Unternehmung eingesetzt wird. Vorhandenes technologisches Wissen in den wissenschaftlichen Einrichtungen, auf das die private Wirtschaft zugreifen kann, führt zur Ausweitung der Forschungsintensität der Unternehmen (komplementäre Beziehung). Das Wissen von Zulieferern und Wettbewerbern scheint dagegen subsitutiv zur eigenen FuE-Tätigkeit der Unternehmen eingesetzt zu werden: Werden Zulieferer bzw. Konkurrenten als wichtige Quelle des innovationsrelevanten Wissens eingeschätzt, so hat es eine Senkung der FuEIntensität der Unternehmen zufolge. Es kann vermutet werden, dass sich in diesem Falle eine Unternehmung entscheidet, auf die selbständige Erarbeitung des Wissens zu verzichten und statt dessen das bereits unternehmensextern erarbeitete, produktionsrelevante Wissen anzueignen (zu internalisieren).

\subsection{Zusammenfassung der Ergebnisse}

Das Ziel dieses Kapitels bestand zum einen darin, den Stand der Forschung zu den Bestimmungsgründen der FuE-Tätigkeit im Wirtschaftssektor nachzuzeichnen, um auf der Grundlage der Gegenüberstellung von Theorie und Empirie robuste Ergebnisse und stilisierte Fakten zu identifizieren und - zum anderen - Erkenntnisse bezüglich der Methodologie der empirischen Innovationsforschung zu gewinnen. Bezüglich der ersten Zielsetzung kann Folgendes als Ergebnis festgehalten werden:

1. Obwohl die Innovationsforschung nach wie vor durch die Erkenntnisse von Schumpeter bzw. die sogenannten Neo-Schumpeter-Hypothesen geprägt ist, liefert der Bestand an empirischer Evidenz wenig Anhaltspunkte für die von Schumpeter postulierte generelle technologische Überle- 
genheit der in konzentrierten Märkten agierenden Großunternehmen. ${ }^{262}$ Zwar wächst mit der Unternehmensgröße die Wahrscheinlichkeit, dass sich eine Unternehmung in FuE-Tätigkeit engagiert. Gemessen am Ressourceneinsatz erweist sich jedoch der Beitrag der Großunternehmen als nicht überproportional.

2. Ursachen für Unterschiede im Innovationsverhalten der gewinngesteuerten Wirtschaftsakteure werden zunehmend in den spezifischen Unternehmenseigenschaften und -strategien gesucht. Diversifizierung, Exporttätigkeit und Profitabilität scheinen die einzelwirtschaftliche Entscheidung für FuE zu begünstigen; der Einfluss dieser Variablen auf die Forschungsintensität der Unternehmen fällt jedoch unterschiedlich aus. Während Diversifizierung und eine höhere Ertragskraft der Wirtschaftsakteure nicht zwangsläufig zur Ausweitung der FuE-Tätigkeit führen ${ }^{263}$, kann davon ausgegangen werden, dass Exporttätigkeit bzw. -intensität einen signifikant positiven Einfluss auf die Forschungsintensität der Unternehmen ausüben.

3. Aus der empirischen Auseinandersetzung mit der zweiten NeoSchumpeter-Hypothese gingen drei - in der weiteren Forschung zu untersuchende - Hypothesen hervor. Erstens liefern die neueren Studien einen schwachen Hinweis auf eine nichtlineare Beziehung zwischen der Marktkonzentration und der Forschungstätigkeit. Zweitens lassen diejenigen Untersuchungen, in denen Forschungsintensitäten und Marktstruktur simultan endogenisiert werden, eine wechselseitige Beziehung zwischen Marktstruktur und Forschungstätigkeit vermuten. Die FuETätigkeit der Unternehmen führt demnach zur Erhöhung der Marktkonzentration, während sich eine hohe Marktkonzentration negativ auf die auf der Branchenebene erfasste Forschungsintensität auswirkt. Drittens geht aus den Untersuchungen des Einflusses der Marktkonzentration auf die Forschungstätigkeit die Schlussfolgerung hervor, dass bei der Suche nach den Bestimmungsgründen der FuE im Wirtschaftssektor stärker auf Branchencharakteristika abgestellt werden sollte. Wird für den Einfluss

262 Vgl. hierzu die Aussage von Cohen/Klepper (1996a), S. 925: „Thus, policy-makers continue to harbour beliefs about the advantages of large firm size in R\&D competition despite the apparent absence of empirical evidence that would support these beliefs."

263 Vgl. hierzu die Aussage von Cohen und Klepper (1992), S. 773: „Firm R\&D intensity has been related to firm characteristics such as size, cash flow, and degree of diversification, but none of these factors play a consistent or sizable role across studies in explaining the heterogeneity in firm R\&D efforts." 
der Nachfrageerwartungen, Aneignungsbedingungen und technologischen Möglichkeiten kontrolliert, dann erweist sich der Einfluss der auf Branchenebene erfassten Marktkonzentration vorwiegend als nicht signifikant.

4. Die Hypothese von Schmookler über die Bedeutung der Nachfrageimpulse für die Entscheidungen der Unternehmen im FuE-Bereich löste die Debatte aus, ob Innovationen primär durch die Nachfrage induziert oder grundsätzlich durch den allgemeinen Fortschritt in der Technologie begünstigt werden. Neuere Untersuchungen, die inputorientierte Indikatoren der Forschungstätigkeit verwenden und Nachfrageänderungen auf der Unternehmensebene erfassen, bestätigen einen positiven Einfluss der Nachfrage auf die Forschungstätigkeit der Unternehmen. Allerdings lässt der Bestand der empirischen Forschung nicht darauf schließen, dass aus der positiven Nachfrageentwicklung stärkere Forschungsimpulse hervorgehen als aus den technologischen Möglichkeiten. ${ }^{264}$

5. Viel Aufmerksamkeit wird in der theoretischen wie empirischen Innovationsforschung der Problematik der positiven Externalitäten der Forschungstätigkeit gewidmet. Sowohl Studien zur Bedeutung der technologischen Möglichkeiten als auch Untersuchungen den Aneignungsbedingungen setzen am gleichen Phänomen der Spillovers an und betrachten es aus zwei verschiedenen Perspektiven. Während Studien zur Aneignungsbedingungen untersuchen, inwieweit besserer Schutz vor unfreiwilligen Spillovers die Forschungstätigkeit stimuliert, überprüfen die Studien zur Bedeutung technologischer Möglichkeiten, inwieweit sich die Fähigkeit der Wirtschaftsakteure, Wissensexternalitäten für unternehmensinterne Zwecke zu internalisieren, positiv auf ihre Forschungstätigkeit auswirkt.

6. Im Lichte der Ergebnisse der in der Tradition Levins et al. (1986) stehenden Untersuchungen zu den Aneignungsmöglichkeiten des auf Erfindungen beruhenden Nutzens bzw. zur Effektivität der unternehmensinternen Schutzmechanismen ${ }^{265}$ kann vom Vorliegen positiver Wissensexternalitäten der FuE-Tätigkeit ausgegangen werden. Das Postulat des negativen Einflusses der unvollständigen Aneignungsmöglichkeiten auf die Entscheidungen der gewinngesteuerten Akteure im FuE-Bereich kann

\footnotetext{
${ }^{264}$ Vgl. Kleinknecht (1996), S. 9: „Supply-side factors tend to be more important for a firm's score on input indicators, whereas demand-pull effects gradually become more important as we move closer to the market, for example, when analyzing sales resulting from innovative products."
}

$265 \mathrm{Vgl}$. Abschnitte 3.4.2.1 und 3.4.2.2. 
insofern als empirisch bekräftigt angesehen werden, als sich in den ökonometrischen Schätzungen ein positiver Einfluss der effektiveren Appropriierung auf die Aufnahme bzw. Ausweitung der FuE-Tätigkeit im Wirtschaftssektor tendenziell bestätigt. Allerdings erkennt die neuere innovationsökonomische Literatur an, dass das intern - dank eigener FuE erarbeitete - wettbewerbsrelevante Wissen nicht nur durch die rechtlichen Schutztitel (Patente, Gebrauchsmuster), sondern auch durch marktstrategische Maßnahmen wie zeitlicher Vorsprung, Geheimhaltung oder Komplexität der Produktgestaltung geschützt werden kann. Rechtliche Schutzmaßnahmen scheinen weniger effektiv als unternehmensinterne Schutzmechanismen zu sein und zudem zunehmend strategische, d.h. über den Schutz eigener Erfindungen hinausgehende, Funktionen zu erfüllen.

7. Die Fähigkeit der Wirtschaftsakteure, Wissensexternalitäten für unternehmensinterne Zwecke zu internalisieren, d.h. von den externen Fortschritten in der Technologieentwicklung zu profitieren, scheint i.d.R. einen positiven Einfluss auf ihre Forschungstätigkeit auszuüben. Bei Untersuchung der Bedeutung der einzelnen externen Wissensquellen zeigt sich jedoch, dass das externe Wissen zum Teil substitutiv und zum Teil komplementär zu eigener FuE der Unternehmung eingesetzt wird. Vorhandenes technologisches Wissen in den wissenschaftlichen Einrichtungen, auf das die private Wirtschaft zugreifen kann, führt zur Ausweitung der Forschungsintensitäten der Unternehmen (komplementäre Beziehung). Das Wissen von Zulieferern und Wettbewerbern scheint dagegen zum Teil subsitutiv zu eigener FuE-Tätigkeit der Unternehmen eingesetzt zu werden: Werden Zulieferer bzw. Konkurrenten als wichtige Quelle des innovationsrelevanten Wissens eingeschätzt, so hat es eine Senkung der FuE-Intensität der Unternehmen zufolge. Es kann vermutet werden, dass sich in diesem Falle eine Unternehmung entscheidet, auf selbständige Erarbeitung des Wissens zu verzichten und statt dessen das bereits unternehmensextern erarbeitete produktionsrelevante Wissen anzueignen (zu internalisieren).

Bezüglich der Methodologie der empirischen Innovationsforschung lassen sich im Zeitablauf folgende Tendenzen feststellen:

1. In den neueren Untersuchungen werden die Bestimmungsgründe der Forschungstätigkeit im Wirtschaftssektor zunehmend auf der Unternehmen- 
sebene untersucht. Zum Teil kann diese Tendenz auf die verbesserte Datenlage zurückgeführt werden: Dank der Etablierung der europaweiten Innovationserhebungen (CIS) sind Unternehmenspanels entstanden, auf die die empirische Innovationsforschung verstärkt zurückgreifen kann. Andererseits wird mit der intensiven Inanspruchnahme der Unternehmensdaten der Tatsache Rechnung getragen, dass bestimmte Konzepte, wie z.B. technologische Möglichkeiten oder Aneignungsbedingungen nur unzureichend auf der Branchenebene abgebildet werden können.

2. In den neueren Untersuchungen wird explizit zwischen Forschungsneigung und Forschungsintensität der Unternehmen unterschieden. Dadurch wird der Erkenntnis Rechnung getragen, dass die einzelwirtschaftlichen Entscheidungen über die Aufnahme der Forschungstätigkeit einerseits und über der Höhe der FuE-Aufwendungen andererseits unter Umständen von verschiedenen Faktoren abhängen können.

3. Insbesondere bei der Untersuchung des Einflusses der Unternehmensgröße kann eine Tendenz zur Ausdifferenzierung der Modellspezifikation festgestellt werden: In den Regressionsgleichungen, in denen die Entscheidung für oder gegen die Aufnahme der Forschungstätigkeit modelliert wird, geht die Unternehmensgröße in linearer Form ein. Dagegen wird bei der Modellierung der Forschungsintensitäten einzelner Unternehmen für den möglichen nichtlinearen Zusammenhang zwischen der Unternehmensgröße und Forschungsintensität kontrolliert.

4. Da in der neueren Literatur über die Defizite einzelner Messgrößen der Innovationstätigkeit verstärkt diskutiert und auf die möglichen Messfehler von Indikatoren eingegangen wurde, empfehlen und verfolgen neuere Studien zunehmend die Verwendung von mehreren Indikatoren der Forschungs- bzw. Innovationstätigkeit. Damit wird für die Robustheit der Ergebnisse verstärkt kontrolliert.

5. Wichtige Erfahrungen sind schließlich bei der Operationalisierung des Konzepts der Spillovers gesammelt worden. Sie werden meist unter Rückgriff auf die Methodologie der grundlegenden Studie von Levin et al. (1987) abgebildet, d.h. unter Anwendung von einzelwirtschaftlichen Angaben zur Wirksamkeit von alternativen Mechanismen zum Schutz innovationsbedingter Technik- und Wissensvorsprünge. Die technologischen Möglichkeiten werden zunehmend auf der Mikroebene erfasst und unter 
Zuhilfenahme von Angaben zur Bedeutung von unternehmensexternen Wissensquellen abgebildet. 
Katarzyna Haverkamp - 978-3-631-75689-8

Downloaded from PubFactory at 01/11/2019 02:56:45AM

via free access 


\section{Kapitel 4}

\section{Vertragsforschung aus theoretischer Perspektive}

Nachdem im vorigen Kapitel bestehende Hypothesen aufgearbeitet worden sind, die die allgemeine Entscheidung für die Aufnahme bzw. Ausweitung der FuE-Tätigkeit im Wirtschaftssektor erklären, gilt es in diesem Kapitel die spezielle Frage zu untersuchen, inwieweit die einzelwirtschaftliche Wahl der Vertragsforschung als einer Koordinationsform für FuE-Aktivitäten theoretisch rekonstruiert werden kann. Hierfür ist es notwendig, Ansätze zu identifizieren, die eine theoriegeleitete Auseinandersetzung mit diesem Phänomen ermöglichen.

Kriterien zur Auswahl des geeigneten theoretischen Analyserahmens werden im folgenden Abschnitt formuliert. Es wird gezeigt, dass institutionenökonomische Ansätze und industrieökonomische Modelle zur Erklärung der Organisationsstruktur im FuE-Bereich herangezogen werden können. Die Erläuterung der Grundstruktur dieser Forschungsansätze und Übertragung der zentralen Konzepte auf den Kontext der Vertragsforschung erfolgen in den Abschnitten 4.2 bis 4.4. Der dieses Kapitel abschließende Abschnitt 4.5 fasst die wesentlichen Ergebnisse der Analyse zusammen. 


\subsection{Auswahl theoretischer Ansätze}

Die primäre Zielsetzung dieses Kapitels liegt darin, das Phänomen der Vertragsforschung einer wirtschaftswissenschaftlichen Analyse zuzuführen, um auf der Basis einer theoriegeleiteten Auseinandersetzung Hypothesen über die Bestimmungsfaktoren der Vertragsforschung abzuleiten. Aus der einzelwirtschaftlichen Perspektive bedeutet die Entscheidung für die Auftragsvergabe eine Entscheidung für die Externalisierung von Teilen eigener Leistungserstellungsprozesse und damit für die marktliche Koordination der Ressourcenallokation. Ein geeigneter Analyserahmen für die theoretische Auseinandersetzung mit dem Phänomen der Auftragsvergabe sollte folglich unter denjenigen Ansätzen gesucht werden, die sich explizit der vergleichenden Analyse der Koordinationsformen ökonomischen Geschehens widmen und Bedingungen für die Wahl bestimmter Organisationsformen identifizieren.

Bei der Suche nach einem geeigneten theoretischen Bezugsrahmen ist ferner zu beachten, dass die gewählten Ansätze die Erfassung derjenigen Aspekte ermöglichen sollen, die sich in der bisherigen empirischen Motivforschung als zentrale Einflussgrößen auf Entscheidungen für bzw. gegen die Auftragsvergabe herausgestellt haben. Im Lichte der vorliegenden empirischen Evidenz ist dies zum einen der Zugriff auf Ressourcen unternehmensexterner Partner als ein zentrales Argument für und Koordinationsprobleme sowie Einschränkungen hinsichtlich der Übermittlung und Exklusivität des erarbeiteten Wissens als Argumente gegen die Auftragsvergabe. ${ }^{266}$

Schließlich kann bei der Abgrenzung des geeigneten theoretischen Bezugsrahmens geprüft werden, welche Ansätze in der bisherigen theoretischen Auseinandersetzung mit der Problematik der Organisationsstruktur im FuE-Bereich verwendet wurden.

Unter Berücksichtigung der erläuterten Kriterien wurden für die Zwecke der vorliegenden Arbeit drei Theorierichtungen ausgewählt, die zur Erklärung der einzelwirtschaftlichen Entscheidung für Vertragsforschung herangezogen werden: Der Transaktionskosten- und Property-Rights-Ansatz sowie die industrieökonomischen Modelle der FuE-Kooperation. Die Auswahl dieser Ansätze soll nachfolgend kurz begründet werden.

Die Erklärungsmuster des Transaktionskosten- bzw. des Property-RightsAnsatzes werden herangezogen, da sich beide Forschungsprogramme explizit

266 Vgl. Rüdiger (2000), S. 45-51. 
der Aufgabe widmen, Bedingungen für die Wahl einzelner Organisationsformen zu identifizieren und bereits bei der Erklärung der Organisationsstruktur im FuE-Bereich Verwendung fanden. ${ }^{267}$ Die Perspektive dieser Ansätze ermöglicht die Beantwortung der Frage, warum eine marktliche Koordination der Ressourcenallokation der hierarchischen Steuerung (vertikalen Integration) vorgezogen wird. Während jedoch im Transaktionskostenansatz die relative Effizienz einzelner Organisationsformen im Hinblick auf die Bewältigung der bei unterschiedlichen Transaktionen anfallenden Koordinationsproblemen untersucht wird, betont der Property-Rights-Ansatz die Bedeutung der spezifischen Ressourcenausstattung einzelner Wirtschaftsakteure. Somit können unter Anwendung dieser Ansätze die zentralen Argumente für bzw. gegen Vertragsforschung erfasst werden.

Die bisher erwähnten Ansätze verdeutlichen zwar, wann bestimmte Transaktionen vertikal in einer Firma integriert bzw. zwischen selbstständigen Akteuren abgewickelt werden, ermöglichen jedoch nur bedingt die Erfassung von Besonderheiten der Ressourcenallokation im FuE-Bereich. Daher ist es geboten, auf die industrieökonomischen Modelle zurückzugreifen, in welchen Kooperationsentscheidungen im FuE-Bereich explizit untersucht werden. In diesen Modellen wird auf einige Konzepte aufmerksam gemacht, die bei der Untersuchung des Phänomens der Vertragsforschung nicht unbeachtet bleiben dürfen.

\subsection{Der Transaktionskostenansatz}

Den Ausgangspunkt transaktionskostentheoretischer Überlegungen bilden Austausch- bzw. Vertragsbeziehungen, die in einer arbeitsteilig organisierten und auf Spezialisierungsvorteile abzielenden Volkswirtschaft zwischen den einzelnen Wirtschaftsakteuren bestehen. ${ }^{268}$ Das Forschungsprogramm des Transaktionskostenansatzes (TKA) zielt auf die Untersuchung der Systeme bzw.

${ }^{267}$ Für die Anwendung des transaktionskostentheoretischen Analyserahmens im Kontext der Vertragsforschung vgl. Rüdiger (2000), Kaltwasser (1994), Dillmann (1996), Dillmann (1997), Schneider/Zieringer (1991), Tapon (1989), Adams/Marcu (2004). Die letztgenannte Studie verweist auch ausdrücklich auf den Property-Rights-Ansatz. Der Modellierung des Phänomens der Vertragsforschungsbeziehungen im Rahmen des Property-Rights-Ansatzes widmet sich auch explizit der Beitrag von Aghion und Tirole (1994).

268 Vgl. Picot/Dietl (1990), S. 178 sowie die Feststellung von Williamson (1990), S. 22: „Die Transaktionskostentheorie formuliert das Problem ökonomischer Organisation als Vertragsproblem." 
Institutionen, die diese Austauschbeziehungen koordinieren sollen. Die vergleichende Analyse der Koordinationsformen ökonomischen Geschehens, wie sie im TKA vorgenommen wird, bedient sich der Transaktion als Basiseinheit. ${ }^{269}$ Es wird davon ausgegangen, dass eine Transaktion als ein Prozess der Übertragung von Verfügungsrechten zwischen zwei Wirtschaftsakteuren ${ }^{270}$ stets von Kosten begleitet wird, deren Höhe von der gewählten Einbettung in ein institutionelles Arrangement abhängt. Das Kernstück der transaktionskostenorientierten Analyse bilden die differenzierende Zuordnung von Transaktionen, die sich in ihren Dimensionen stark voneinander unterscheiden, zu den Steuerungsund Überwachungssystemen (governance structures), unter Berücksichtigung von spezifischen, von Neoklassik abweichenden Verhaltensannahmen. ${ }^{271}$

In diesem Abschnitt sollen zentrale Erkenntnisse und Argumentationsbausteine des Transaktionskostenansatzes auf den Kontext der Vertragsforschung übertragen werden. Hierfür werden zunächst die für die nachfolgende Interpretation der Vertragsforschung relevanten Grundkonzepte des Transaktionskostenansatzes erläutert (Abschnitte 4.2.1 bis 4.2.2). Dies ist notwendig, da der TKA als verbale Theorierichtung eine Vielzahl von distinktiven Begriffen entwickelt hat, die eine einführende Erläuterung unumgänglich macht. Darauf aufbauend erfolgt in Abschnitt 4.2.3 die Analyse des Phänomens der Vertragsforschung aus der Perspektive des Transaktionskostenansatzes.

\subsubsection{Grunderkenntnisse von Coase}

Nachfolgend werden kurz die wichtigsten Erkenntnisse von Ronald Coase dargestellt, welche die Grundlage für die transaktionskostenorientierte Theorierichtung bilden. Auf den Coaseschen Überlegungen basiert letztlich die Konzeptualisierung von Unternehmen und Märkten als alternative Koordinationsformen des ökonomischen Geschehens.

In seinem im Jahre 1937 veröffentlichten Beitrag „The Nature of the Firm "ging Coase von der Beobachtung aus, dass die neoklassische Theorie keine hinreichende Erklärung für die Existenz einer Unternehmung liefern konnte. ${ }^{272}$ In einer Welt vollkommener Märkte würden alle Transaktionen über den Markt

269 Vgl. Williamson (1990), S. 47, Williamson (1993), S. 16.

270 Zur Definition der Transaktion vgl. Abschnitt 4.2.2.1.

271 Vgl. Williamson (2000), S. 599 und 602.

272 Vgl. Martiensen (2000), S. 107 f., Chung (1998), S. 50. 
abgewickelt, es gäbe keine Rechtfertigung für eine nicht preisgesteuerte Allokation der Ressourcen, wie sie in der Unternehmung stattfindet. Den Grund für die Entstehung von Unternehmen sah Coase in der nicht friktionslosen Funktionsweise des Marktes. ${ }^{273} \mathrm{Da}$ die Inanspruchnahme des Preismechanismus mit Kosten verbunden sei, können in einem Unternehmen kostenspielige Marktvorgänge durch unternehmensinterne Steuerung ersetzt werden.

Die Coasesche Hinterfragung der neoklassischen Annahme vollkommener Märkte verdeutlichte die Bedeutung der Transaktionskosten für die Analyse der Koordinationsformen ökonomischen Geschehens. Während in der neoklassischen Theorie der auf den reibungslos funktionierenden Märkten zustande kommende Preis als der effizienteste Steuerungsmechanismus von wirtschaftlichen Aktivitäten angesehen wurde, erkannte Coase, dass die Koordinationsaufgabe anstelle von Märkten von Unternehmen übernommen werden kann. In der Coaseschen Analyse wurden Markt und Unternehmung somit zu alternativen Institutionen zur Steuerung der Allokation der den Knappheitsverhältnissen unterliegenden Ressourcen. ${ }^{274}$ Während in einem Unternehmen hierarchische Verhältnisse vorherrschen und die Koordination der individuellen Handlungen über eine Weisung erfolgt, findet auf dem Markt eine dezentrale Abstimmung der einzelwirtschaftlichen Pläne statt, wobei die Änderung der relativen Preise Anpassungsreaktionen hervorruft. ${ }^{275}$

Coase erkannte jedoch auch, dass die Inanspruchnahme beider Steuerungssysteme mit indiosynkratischen Kosten verbunden ist. Wäre dies nicht der Fall, d.h. wäre nur eine Abwicklung der Transaktion über den Markt ressourcenintensiv, würden alle Wirtschaftsaktivitäten von einem einzigen Unternehmen (kostenfrei) koordiniert. Den Grund für die Entstehung der Kosten unternehmensinterner Steuerung von Wirtschaftsaktivitäten sah Coase in der zunehmenden Komplexität der arbeitsteiligen Verflechtung in wachsenden Unternehmen. ${ }^{276}$ Bei steigender Unternehmensgröße - so seine These - erweist es sich als zunehmend schwer, die Ressourcen entsprechend ihrer produktivsten

${ }^{273}$ Vgl. dazu Coase (1937), S. 390: „The main reason why it is profitable to establich a firm would seem to be that there is a cost of using the price mechanism."

${ }^{274}$ Vgl. dazu Coase (1937), S. 388: „Outside the firm, price movements direct production, which is co-ordinated through a series of exchange transations on the market. Within a firm, these market transactions are eliminated and in place of the complicated market structure with exchange transactions is subsituted the entrepreneu-co-ordinator, who directs production. It is clear that these are alternative methods of co-ordinating production."

275 Vgl. Erlei/Leschke/Sauerland (1999), S. 66.

276 Vgl. Kiwit (1994), S. 117. 
Verwendung einzusetzen. Daher verliert die hierarchische Steuerung mittels Anweisung an einem bestimmten Punkt ihren Vorteil gegenüber der dezentralen Marktlösung; eine ökonomisch fundierte Grenze der Unternehmung kann anhand eines Grenzkostenvergleichs beider alternativer Steuerungsmechanismen bestimmt werden. ${ }^{277}$

\subsubsection{Williamsons vertragstheoretische Weiterentwick- lung}

Während Coase die natürliche Grenze einer Unternehmung in der Koordinationsfähigkeit des Unternehmers ${ }^{278}$ sah, d.h. in der Fähigkeit eines Entrepreneurs, nur eine begrenzte Menge von internalisierten Tauschvorgängen effizienter zu gestalten, ist es Williamson gelungen, die Eigenschaften von Transaktionen zu ermitteln, anhand derer eine Zuordnung einzelner Transaktionen zu den jeweiligen Koordinationsformen möglich ist. Williamson unterschied drei Attribute von Transaktionen, die nach seiner Ansicht die Höhe der jeweils anfallenden Tauschkosten determinieren: Faktorspezifität (asset specificity), Häufigkeit der Tauschvorgänge und Unsicherheit. ${ }^{279}$ Er behauptete, dass eine kosteneinsparende Zuordnung der jeweiligen Transaktionen zu den sich in den Anreiz- und Anpassungseigenschaften unterscheidenden alternativen Beherrschungs- und Überwachungssystemen den richtigen Ansatz biete für die vergleichende Beurteilung institutioneller Alternativen zwischen dem klassischen Markttausch und der zentralisierten hierarchischen Organisation. ${ }^{280}$

\subsubsection{Zum Begriff der Transaktionskosten}

Bevor in den nächsten Abschnitten auf die Eigenschaften der Transaktionen (Abschnitt 4.2.2.2) und der einzelnen Koordinationsformen ökonomischen Geschehens eingegangen wird (Abschnitt 4.2.2.3), sollen zunächst die Begriffe

277 Vgl. dazu die Aussagen von Coase (1937), S. 394 f.: „[...] it may be that as the transactions which are organised increase, the entrepreneur fails to place the factors of production in the uses where their value is greatest, that is, fails to make the best use of the factors of production" und "Naturally, a point must be reached where the costs of organising an extra transaction within the firm are equal to the costs involved in carrying out the transaction in the open markt, or, to the costs of organising by another entrepreneur."

${ }^{278} \mathrm{Zu}$ diesem Ausdruck vgl. Kiwit (1994), S. 117.

279 Vgl. Williamson (1990), S. 59.

280 Vgl. Williamson (1990), S. $47 \mathrm{f}$. 
Transaktion und Transaktionskosten (TK) definiert werden, da sie in der Literatur unterschiedlich abgegrenzt und in verschiedenen Bedeutungszusammenhängen verwendet werden. ${ }^{281}$ Bei der Wahl der Definitionen, die den nachfolgenden Ausführungen zugrunde gelegt werden, gilt es zu beachten, dass die gewählten Abgrenzungen es ermöglichen sollen, die Erkenntnisse des Transaktionskostenansatzes auf die Problematik der Vertragsforschung zu übertragen.

Als eine Transaktion definiert Williamson den Transfer von Gütern und Dienstleistungen über eine technologisch separierbare Schnittstelle. ${ }^{282}$ Mit dieser Definition wird die oft implizit unterstellte Identität einer Transaktion und einem auf dem Markt stattfindenden Tauschvorgang verlassen: Da es sich um die Weitergabe von Transaktionsobjekten handelt, die sowohl unternehmensextern als auch unternehmensintern erfolgen kann, wird in dieser Definition der Geltungsbereich des Begriffes Transaktion auch auf diejenigen Transfers ausgeweitet, die innerhalb einer hierarchischen Organisation stattfinden. Als Nachteil muss jedoch eingestuft werden, dass in dieser Abgrenzung Güter und Dienstleistungen - und nicht die an ihnen bestehenden Verfügungsrechte (property rights) - als Transaktionsobjekte fungieren. Daher wird die Williamsonsche Definition zugunsten einer anderen Abgrenzung aufgegeben, die als Ausgangspunkt sowohl transaktionskostenorientierter als auch die Rolle der Property Rights betonender Theorierichtungen aufgefasst werden kann. Eine solche Definition wurde von Martiensen vorgeschlagen: Eine Transaktion ist ein Prozess der Einrichtung, Änderung und/oder Übertragung von Verfügungsrechten. ${ }^{283}$ Wenn im Folgenden von Transaktionen auf dem FuEDienstleistungsmarkt gesprochen wird, ist dabei die Einrichtung bzw. Übertragung von Property-Rights an innovationsrelevantem Wissen als einem Ergebnis der FuE-Tätigkeit gemeint.

In seinem grundlegenden Beitrag von 1937, in dem die Bedeutung der Friktionen im Marktgeschehen herausgestellt wurde, verwendete Coase nicht den Begriff der Transaktionskosten, sondern den der Vermarktungskosten (marketing costs). Gemeint war damit jedoch stets der Ressourcenverbrauch, der bei der Abwicklung einer Transaktion über den Markt anfällt. Im Verlauf späterer Forschung erwies es sich jedoch als vorteilhaft, den Begriff der Transaktionskosten - ähnlich wie den der Transaktion - nicht nur im Bezug auf das Marktgeschehen zu verwenden, sondern seine Geltung auch auf den bei der unternehmensinternen Koordination von ökonomischen Aktivitäten nötigen

\footnotetext{
281 Vgl. Kiwit (1994), S. $111 \mathrm{f}$.

282 Williamson (1990), S. 1, Williamson (1996), S. 58.

283 Vgl. Martiensen (2000), S. 272.
} 
Ressourcenverbrauch auszuweiten. Somit wurde mit Transaktionskosten derjenige Ressourcenverbrauch umschrieben, der jede Transaktion begleitet, und zwar unabhängig davon, ob diese Transaktion auf dem Markt oder unternehmensintern abgewickelt wird. In diesem Sinne wurden die Transaktionskosten von Arrow als "cost of running the economic system" ${ }^{284}$ oder von Demsetz als „cost of organizing resources" ${ }^{485}$ definiert.

Auf diese sehr weite Fassung des Begriffes der Transaktionskosten (TK) als "Betriebskosten des Wirtschaftssystems" wird im Folgenden zugunsten einer Abgrenzung von Tietzel verzichtet. Tietzel definiert Transaktionskosten als im Zusammenhang mit der Bestimmung, Übertragung, Überwachung und Durchsetzung von Verfügungsrechten entstehenden Ressourcenverbrauch. ${ }^{286}$ Damit umschließen TK im Kontext der Vertragsforschung sowohl die Such- und Informationskosten, die bei der Anbahnung eines FuE-Vertrages entstehen, die Verhandlungskosten, die bei dem Abschluss des Vertrages anfallen als auch die Überwachungs- und Durchsetzungskosten, welche die Abwicklung des Vertrages begleiten.

\subsubsection{Einflussgrößen auf die Höhe der Transaktionskosten}

Grundlegend für die Entstehung und die Höhe der Transaktionskosten sind in der Williamsonschen Transaktionskostenökonomik neben drei Eigenschaften der Transaktionen zwei zentrale Annahmen bezüglich des Verhaltens der Wirtschaftsakteure: begrenzte Rationalität und Opportunismus. Unter begrenzter Rationalität wird das Unvermögen der Individuen verstanden, alle ihnen zufließenden Informationen zu erfassen, zu verarbeiten, zu speichern und in einer Entscheidungssituation effektiv umzusetzen. ${ }^{287}$ Dem intendiert rationalen Verhalten der Wirtschaftsakteure sind somit aufgrund ihrer eingeschränkten kognitiven Kapazität Grenzen gesetzt. ${ }^{288}$ Die Wirtschaftsakteure verfügen über keine perfekte Prognosefähigkeit, können die Umweltveränderungen nicht antizipieren und infolge dessen keine vollständigen Verträge formulieren. ${ }^{289}$

\footnotetext{
${ }^{284}$ Arrow (1969), S. 48, zitiert nach Williamson (1996), S. 5.

285 Demsetz (1988), S. 144.

286 Vgl. Tietzel (1981), S. 211.

287 Vgl. Chung (1998), S. 62.

288 Vgl. Williamson (1993), S. 11.

289 Vgl. Chung (1998), S. 62.
} 
Die Unvollständigkeit der Verträge stellt jedoch ein grundlegendes Problem nur in Verbindung mit der zweiten Verhaltensannahme der Williamsonschen Transaktionskostenökonomik dar: dem Opportunismus der Wirtschaftsakteure, verstanden als ihre Neigung, Eigeninteressen unter Anwendung von List und Tücke zu verfolgen. ${ }^{290}$ Bei Annahme potenziell opportunistischen Verhaltens von Transaktionspartnern im Sinne eines egoistischen Ausnutzens von günstigen Positionen ${ }^{291}$ fungieren unvollständige Verträge als Träger von Gefahren wie einseitige Leistungszurückhaltung (shirking), moralische Versuchung (moral hazard) oder Erpressung (hold-up) ${ }^{292}$ Aufgrund dieser Gefahren, die je nach Art der Transaktionen verschieden stark ausfallen können, bedarf es nach Ansicht Williamsons unterschiedlicher Koordinationsformen zwischenmenschlicher Austauschbeziehungen, die die Aufgabe von Kontroll- und Überwachungssystemen (governance structures) erfüllen können.

Unter den Eigenschaften der Transaktionen nimmt die Faktorspezifität (asset specificity) in der Williamsonschen Transaktionskostenökonomik eine zentrale Stellung ein. Von Faktorspezifität wird dann gesprochen, wenn im Vorfeld einer bestimmten abzuwickelnden Transaktion Investitionen getätigt werden, die ursprünglich homogene Inputfaktoren so verändern, dass sie in alternativer Verwendung entweder keinen oder nur geringen Wert aufweisen; der Wert der investierten Ressourcen hängt nun unmittelbar von dem Zustandekommen der antizipierten Transaktion ab. ${ }^{293}$ Transaktionsspezifität manifestiert sich folglich in der Bindung von dauerhaften Investitionen an einen gut definierten Transaktionsvorgang. ${ }^{294}$

Tritt einer der Vertragspartner in Vorleistung, indem er die für die Abwicklung einer Transaktion notwendige hochspezifische Investitionen tätigt, führt das zur Entstehung von einseitiger Abhängigkeit, dessen Grad von der Differenz des Wertes abhängt, den die investierten Ressourcen in der ursprünglich geplanten und in der nächstbesten Verwendungsmöglichkeit erzielen können. Diese Differenz wird als Quasi-Rente bezeichnet. ${ }^{295}$ Sie kann den Wert von Null einnehmen, wenn es sich bei den nötigen Investitionen z.B. um Mehrzwecktechnologien handelt. Sie kann aber auch der Höhe der getätigten In-

${ }^{290}$ Zur eingehenden Erläuterung der Definition von Opportunismus vgl. Wx/illiamson (1990), S. $54 \mathrm{ff}$.

${ }^{291} \mathrm{Zu}$ dieser Definition von Opportunismus vgl. Kaas/Fischer (1993), S. 687.

292 Vgl. Williamson (2000), S. 601.

293 Vgl. Chung (1998), S. 64.

294 Vgl. Williamson (1990), S. 63.

295 Zum Konzept der Quasi-Rente vgl. Klein/Crawford/Alchian (1978), S. 298 ff. 
vestitionen entsprechen, wenn hochspezifische Aufwendungen (Einzwecktechnologien) involviert sind. Je höher diese Differenz ausfällt, desto komplexere Überwachungs- und Kontrollsysteme werden für die Absicherung der Vertragsbeziehung benötigt, da die aufgrund von Faktorspezifität entstandene Machtasymmetrie bzw. einseitige Abhängigkeit eine Gefahr der Ausbeutung durch den Transaktionspartner in sich birgt. Daher stellt Williamson die Faktorspezifität als diejenige Dimension einer Transaktion heraus, die die ausschlaggebende Bedeutung bei der Wahl einer institutioneller Einbettung für einen Vertrag einnimmt: Dort, wo die Faktorspezifität vernachlässigt werden kann, stellt der Markttausch die leistungsfähigste bzw. effizienteste Lösung dar. Im Umfeld nichtumschichtbarer Vorlaufinvestitionen erweist sich jedoch eine preisgesteuerte Vertragsabwicklung als unterlegen gegenüber der internen Organisation. ${ }^{296}$

Die Ausnutzung der Ausbeutungsmöglichkeit, die aufgrund von Faktorspezifität entsteht, wird in der institutionenökonomischen Literatur als hold-up (Überfall auf die Quasi-Rente) bezeichnet. Hold-up kann sich beispielweise in einem Versuch eines der Transaktionspartner manifestieren, die ursprünglich vereinbarten Vertragsmodalitäten zu seinen Gunsten zu verändern, nachdem der Tauschpartner die hochspezifische Investitionen getätigt hat. Die Entstehung der Hold-up-Gefahr lässt sich folglich auf die Tatsache zurückführen, dass bei Tauschvorgängen, die sich auf transaktionsspezifische Investitionen stützen, die anonyme (neoklassische) Marktbeziehung durch eine Beziehung ersetzt wird, in welcher die beidseitige Identität der Transaktionspartner die entscheidende Rolle spielt. Aus der ursprünglichen Situation eines beidseitigen Polypols entsteht durch die transaktionsspezifische Investition die Marktstruktur eines Monopsons bzw. Monopols. ${ }^{297}$ Die Wandlung der Marktform im Anschluss an die Durchführung von spezifischen Investitionen bezeichnet Williamson als fundamentale Transformation ${ }^{298}$, die entstandene Situation der wechselseitigen Abhängigkeit als lock-in-Effekt.

Die Häufigkeit, mit der Transaktionen durchgeführt werden, hat als zweite „Dimension“ des Williamsonschen Ansatzes einen starken Einfluss auf die Höhe der Transaktionskosten. Tendenziell gilt, dass isolierte Tauschvorgänge gut über den Markt abgewickelt werden können. Eine hohe Bedeutung erlangt Häufigkeit vorwiegend im Zusammenspiel mit der bereits erläuterten Faktorspezifität. Transaktionen, die sich auf hochspezifische Investitionen stützen, verlangen nach komplexen institutionellen Regelungen, die i.d.R.

296 Vgl. Williamson (1990), S. 88.

297 Vgl. Erlei/Leschke/Sauerland (1999), S. 182, Williamson (1989), S. 145.

${ }^{298}$ Zur Problematik der fundamentalen Transformation vgl. Williamson (1990), S. $70 \mathrm{ff}$. 
selbst stark ressourcenintensiv sind. Die beträchtlichen Fixkosten der komplexen Steuerungs- und Überwachungssysteme können nur bei großer Nutzungshäufigkeit dieser Systeme bzw. bei hohen Transaktionsvolumina amortisiert werden. Williamson stellt hierzu fest: „Nur häufig wiederholte Transaktionen werden ein hochspezialisiertes Beherrschungs- und Überwachungssystem stützen“. 299

Unsicherheit kann als Unmöglichkeit der Wirtschaftsakteure aufgefasst werden, etwaige Umweltzustände umfassend zu prognostizieren und mit subjektiven Eintrittswahrscheinlichkeiten zu belegen. ${ }^{300}$ Unsicherheit, die sich aus einer Unvorhersehbarkeit bzw. Unwägbarkeit der zukünftigen Umweltentwicklungen ergibt, führt dazu, dass vollkommene Spezifizierung von Verträgen unmöglich wird und sich adaptive, sequentielle Entscheidungen in der Vertragsabwicklungsphase als erforderlich herausstellen. Da sich jedoch eine nachträgliche Anpassung der Vertragsmodalitäten an eine aufgetretene umweltbedingte (d.h. exogene) Störung als schwierig erweisen kann, da sich in diesem Falle opportunistisch handelnde Transaktionspartner über eine Veränderung einigen müssen ${ }^{301}$, ist die Transaktionsunsicherheit sowohl durch die Entwicklung der Umwelt, als auch durch das Verhalten der Tauschpartner bedingt. ${ }^{302}$ Im Allgemeinen gilt: Eine hohe Unsicherheit begünstigt die Entscheidung für eine interne Steuerung (vertikale Integration).

Mit den erläuterten Einflussgrößen auf die Höhe der Transaktionskosten in Verbindung mit spezifischen Verhaltensannahmen lässt sich die Entscheidung für eine vertikale Integration der Transaktion in eine Firma begründen: Immer dann, wenn wiederholte Transaktionen durch hochspezifische Investitionen in Vermögensgegenstände gestützt werden, und wenn Unsicherheit über die zukünftige Umweltentwicklung vorliegt, ist eine unternehmensinterne hierarchische Steuerung effizienter. ${ }^{303} \mathrm{Im}$ Falle der vertikalen Integration gibt es keinen Transaktionspartner mehr, der einen Ausbeutungsversuch (hold-up) unternehmen könnte. Die transaktionsspezifischen Quasi-Renten sind nicht mehr gefährdet; folglich gibt es keinen Anhaltspunkt für volkswirtschaftlich unerwünschte Unterinvestitionen in spezifisches Kapital.

\footnotetext{
299 Williamson (1990), S. 82.

$300 \mathrm{Vgl}$. Chung (1998), S. 67.

301 Vgl. Chung (1998), S. 67.

$302 \mathrm{Vgl}$. Terberger (1994), S. 66.

303 Vgl. Chung (1998), S. 67.
} 


\subsubsection{Komparative Analyse der Koordinationsformen}

Alternative Mechanismen der Ressourcenallokation - Märkte, Unternehmen und sog. hybride Formen - unterscheiden sich laut Williamson im Hinblick auf ihre indiosynkratischen Anreiz- und Anpassungseigenschaften, d.h ihre Fähigkeit, auf unvermeidbare exogene Störungen (disturbances) in der Transaktionsbeziehung zu reagieren. Nachfolgend werden die aus diesem Winkel betrachteten Unterschiede zwischen den institutionellen Arrangements näher untersucht.

Williamson geht in seinen Überlegungen von der Hayekschen Beobachtung aus, dass ökonomische Probleme als Konsequenz von Umweltveränderungen auftreten $^{304}$ und untersucht folglich die Fähigkeit der einzelnen Koordinationsformen ökonomischen Geschehens, auf Veränderungen im Transaktionsumfeld zu reagieren. Im Bereich homogener Ressourcen stellt er weitgehend eine Überlegenheit der marktlichen Koordination fest. Da auf dem Markt Austauschprozesse aus spontanen Interaktionen zwischen unabhängigen und gemäß ihren individuellen Präferenzen handelnden Akteuren erwachsen ${ }^{305}$, sind alle beteiligten Wirtschaftseinheiten an einer effizienten Anpassung an veränderte Rahmenbedingungen unmittelbar interessiert. Auf Veränderungen der relativen Preise reagieren sie dezentral mit autonomen Anpassungsreaktionen (Anpassungsfähigkeit vom Typ A), handelnd auf der Grundlage eigener Nutzenmaximierung. ${ }^{306}$ Der komparative Vorteil des Marktes im Bereich homogener Ressourcen begründet sich folglich sowohl im Hinblick auf die Anpassungsflexibilität und -zeit als auch auf die entfaltete Anreizwirkung.

Seinen komparativen Vorteil im Hinblick auf die Anpassungszeit verliert der Markt jedoch dann, wenn wechselseitige Abhängigkeiten aufgrund von Faktorspezifität bestehen. In einer solchen Situation bedarf es im Falle wesentlicher Änderungen der Rahmenbedingungen einer synchronisierten Anpassung zweier oder mehrerer sich in einer Transaktionsbeziehung befindender Akteure. Dies kann sich insbesondere vor dem Hintergrund opportunistischer Spielräume als schwierig und zeitaufwendig erweisen. Hierarchische und nicht dezentral stattfindende Anpassungsprozesse und -maßnahmen sind dann effizienter. Die langwierigen Verhandlungen zwischen den Transaktionspartnern können durch An-

304 Vgl. Hayek (1945), S. 524.

305 Vgl. Fritsch/Wein/Ewers (1996), S. 4.

${ }^{306}$ Preise stellen laut Williamson im Bereich homogener Güter „suffiziente Maßzahlen“ dar, d.h. liefern die notwendige Entscheidungsbasis und ermöglichen schnelle Anpassungsreaktionen, vgl. dazu Williamson (1991a), S. 20. 
weisungen der Unternehmensleitung ( $f i a t$ ) ersetzt werden (Anpassungsfähigkeit vom Typ B). ${ }^{307}$

Der Zuwachs an Anpassungsfähigkeit vom Typ B in einer hierarchischen Beherrschungsstruktur geschieht jedoch auf Kosten der reduzierten Anreizstärke. Williamson geht davon aus, dass die Anreizstärke des Marktes aufgrund der direkten Verknüpfung zwischen einer Leistung und der Gegenleistung besteht. Diese direkte Verknüpfung geht jedoch in einer zentral gesteuerten Organisation (nach der vertikalen Integration) verloren: Eine marktähnliche, ergebnisorientierte Belohnung individueller Leistungsanstrengungen wird erschwert.

Dieser Effekt lässt sich auch durch die Einführung weitgehender unternehmensinterner Simulation des Marktes nicht ausgleichen. Wäre eine Unternehmung in der Lage, marktähnliche Koordination innerhalb der eigenen Organisationsstruktur einzuführen, und nur in Ausnahmefällen (d.h. selektiv), angesichts wesentlicher Umweltstörungen, auf die Weisungsbefugnis zurückzugreifen, wäre dies optimal. Williamson behauptet jedoch, dass die Weisungsinstanz eine Neigung zu einer überhöhten Eingriffsintensität aufweist. Berücksichtigt man zudem, dass der zentralisierten Unternehmensleitung nicht alle - dezentral vorliegenden - Informationen zur Verfügung stehen, d.h. dass es im Rahmen einer Unternehmung mit mehreren Hierarchiestufen Informationsvermittlungsprobleme geben kann, dann können die Interventionen der Unternehmensleitung fehlerhafte, erfolgsschmälernde Eingriffe zur Folge haben. ${ }^{308}$

Im Ergebnis sind es die innerbetrieblichen Anreizprobleme und die Unmöglichkeit der selektiven Intervention, die in der Williamsonschen Transaktionskostenökonomik zur Entstehung von „Bürokratiekosten“ führen. ${ }^{309}$ Eine Zusammenfassung obiger Ausführungen zu den komparativen Vor- und Nachteilen einzelner Organisationsformen kann der Tab. 4.1 entnommen werden.

Um die Entscheidung zwischen Markt um Hierarchie treffen zu könnnen, bedarf es jedoch nach Ansicht von Williamson im Allgemeinen einer gleichzeitigen Berücksichtigung von Produktions- und Transaktionskosten. Weitere Vorteile der Märkte im Bereich nichtspezifischer Ressourcen liegen nämlich in der Bündelung von Nachfrage und Angebot, wodurch sich Größen- und Skaleneffekte realisieren lassen. Bei Homogenität der Einsatzfaktoren ist die interne Herstellung eines Gutes für den Eigengebrauch ineffizienter, da in diesem Falle

307 Vgl. Chung (1998), S. 68.

308 Zur Problematik der überhöhten Eingriffsintensität vgl. insbesondere Erlei/Leschke/Sauerland (1999), S. 186 f.

309 Vgl. Chung (1998), S. $71 \mathrm{f}$. 
Tabelle 4.1: Eigenschaften von Markt, Unternehmen und hybriden Koordinationsformen

\begin{tabular}{lccc}
\hline \hline & \multicolumn{3}{c}{ Beherrschungs- und } \\
& Überwachungsmechanismus \\
\hline & Markt & Hybridform & Hierarchie \\
\hline \hline Anpassungsfähigkeit vom Typ A & ++ & + & 0 \\
Anpassungsfähigkeit vom Typ B & 0 & + & ++ \\
Anreizstärke & ++ & + & 0 \\
administrative Kontrollmöglichkeiten & 0 & + & ++ \\
\hline \hline
\end{tabular}

$0=$ schwach $;+=$ weniger stark, $++=$ stark

Quelle: Williamson (1991b), S. 281.

ein komplexes, d.h. kostenintensiveres Beherrschungs- und Überwachungssystem (eine Unternehmung) eingesetzt wird, um die Allokation von homogenen Faktoren zu steuern. Bei steigender Faktorspezifität lässt dieser Vorteil des Marktes allerdings nach, da in diesem Falle Spezialisierungsvorteile realisiert und die Kosten der Nutzung eines komplexen Steuerungssystems kompensiert werden können.

\subsubsection{Transaktionskostenorientierte Analyse der Ver- tragsforschung}

Nachdem in den vorangegangenen Abschnitten die grundlegenden Argumentationsbausteine der Williamsonschen Transaktionskostenökonomik erläutert wurden, erfolgt nun eine Übertragung der dargestellten Konzepte auf die Problematik der einzelwirtschaftlichen Wahl der institutionellen Produktionsstruktur im FuE-Bereich. Diese Vorgehensweise soll es ermöglichen, transaktionskostengeleitete Bedingungen für die Wahl der Vertragsforschung als einer Abwicklungsalternative für eine FuE-Leistung zu identifizieren.

Aus der Perspektive der Transaktionskostenökonomik kann Vertragsforschung als ein Prozess der Einrichtung und Übertragung der Property-Rights an dem im Rahmen einer FuE-Dienstleistung erarbeiteten Wissen interpretiert werden. Die Vergabe von FuE-Aufträgen an andere institutionelle Akteure ist einer 
Entscheidung für die Marktlösung, d.h. einer preisgesteuerten Abwicklung der FuE-Prozesse, gleichzusetzen. Die entscheidende Frage ist, welche Faktoren die Entscheidung für die Marktlösung begünstigen bzw. verhindern können.

\subsubsection{Zur Bedeutung der Vertragsunvollständigkeit}

Zunächst kann davon ausgegangen werden, dass bei der Auftragsvergabe im FuE-Bereich die Problematik der Unvollständigkeit von Verträgen eine besondere Rolle spielt. Da es sich bei Forschungstätigkeit um einen Produktionsprozess mit einem hohen Grad an prozessinterner Unsicherheit handelt, dürfte eine vollständige Spezifizierung der von einer Seite zu erbringenden Leistung mit erheblichen Problemen verbunden sein. Teece erläutert in einer synthetischen Weise die im Zusammenhang mit der Spezifizierung von FuE-Verträgen entstehenden Probleme. ${ }^{310}$ Erstens müssen in der Vertragsvorbereitungsphase dem potenziellen Tauschpartner Informationen vermittelt werden, die wettbewerbsrelevante Hinweise enthalten können. Angesichts der Tatsache, dass dabei auch Wissen vermittelt werden kann, das Eigenschaften von öffentlichen Gütern aufweist und folglich nur eingeschränkt vor unentgeltlicher Weiterverwendung geschützt werden kann, dürfte sich dieser Umstand bereits im Vorfeld einer antizipierten Beziehung transaktionshemmend auswirken. Zweitens führt die stochastische Natur der FuE-Tätigkeit dazu, dass Vertragsmodalitäten nicht ex-ante exakt festgelegt werden können, sondern parallel zu Erkenntnisfortschritten in der Vertragsabwicklungsphase in sequentiellen Entscheidungsprozessen verändert und angepasst werden müssen. ${ }^{311}$ Drittens können erhebliche Probleme bei der vertraglichen Festlegung des Preises entstehen, da aufgrund der prozessinternen Unsicherheit die effektiven Kosten der Forschungsleistung schwer zu ermitteln sind.

Insbesondere die ersten beiden von Teece angesprochenen Probleme der Vertragsfestlegung machen einen weiteren Aspekt deutlich. Die Notwendigkeit eines intensiven Austausches sowohl in der Vertragsvorbereitung- als auch während der Vertragsabwicklungsphase birgt Gefahren für beide Transaktionspartner insofern in sich, als hierbei eine gegenseitige Übermittlung von innovationsrelevantem Wissen erfolgen muss. Im Interesse beider Parteien liegt es dabei nicht, die über das notwendige Mindestmaß hinausgehenden Wis-

\footnotetext{
$310 \mathrm{Vgl}$. Teece (1988), S. $260 \mathrm{f}$.

311 Zur Notwendigkeit der sequentiellen Anpassung der Vertragsmodalitäten vgl. auch Pisano (1990), S. 157 f., Rüdiger (2000), S. 107.
} 
sensvorsprünge und Erfahrungen an den jeweiligen Partner weiterzuleiten. ${ }^{312}$ Daher kann vermutet werden, dass für die Vertragsparteien Instrumente zum Schutz eigener Wissensvorsprünge eine besondere Rolle spielen (Hypothese 1). ${ }^{313}$

Wird von der Kondition eines unvollständigen Vertrages ausgegangen, so bedeutet dies, dass „nicht unerheblicher Raum für Opportunismus verbleibt “314, sowohl auf der Seite des Auftraggebers als auch des FuE-Dienstleisters. Aus der Sicht des Auftraggebers besteht eine Gefahr darin, dass der effektive Forschungseinsatz des Auftragnehmers nicht bzw. nur unzureichend beobachtet werden kann. ${ }^{315}$ Eine unter dem notwendigen Niveau liegende FuE-Investition des FuE-Dienstleisters kann folglich nicht ausgeschlossen werden. Zwei Instrumente zur Bewältigung der Gefahr einer Leistungszurückhaltung seitens des FuE-Anbieters stehen dem Auftraggeber zur Verfügung. Zum einen können umfangreiche Kontroll- und Überwachungssysteme aufgebaut werden. Da jedoch Kontrollsysteme ein kostenintensives institutionelles Arrangement darstellen, kann vermutet werden, dass bei der Wahl eines FuE-Dienstleisters auf die Partner zurückgegriffen wird, zu denen Vertrauen besteht, welches beispielweise in informellen und formellen Kooperationsbeziehungen aufgebaut werden konnte (Hypothese 2). Besteht Vertrauen zwischen den involvierten Tauschpartnern, so können Verhaltensunsicherheiten und in der Folge Kontrollkosten reduziert werden. ${ }^{316}$

Eine weitere Gefahr, die infolge der Unmöglichkeit exakter Spezifizierung der Vertragsbedingungen entsteht, bezieht sich auf die Art der Verwendung des im Rahmen einer FuE-Dienstleistung erarbeiteten Wissens. Der Vorteil einer FuE-Dienstleistung liegt seitens des Auftragnehmers darin, dass während der auszuführenden Arbeiten spezifische Erfahrungen im Umgang mit bestimmten Problemstellungen gesammelt werden und distinktive Kompetenzen aufgebaut werden können. Während folglich der Auftraggeber durch Auftragsvergabe Lernkurveneffekte verliert, baut der Auftragnehmer internes Wissen auf. $^{317}$ In der Konsequenz ist davon auszugehen, dass Unternehmen, die FuELeistungen bereits im Auftrag vergeben haben, zunehmend darauf angewiesen

312 Vgl. Pisano (1991), S. 245.

$313 \mathrm{Vgl}$. Teece (1988), S. 260.

314 Rüdiger (2000), S. 104.

$315 \mathrm{Vgl}$. hierzu ausführlicher Abschnitt 4.3.4.2.

316 Vgl. Tapon/Thong (1997), S. 28 f., Rüdiger (2000), S. 108, Kaltwasser (1994), S. 175.

317 Vgl. Kommission der Europäischen Gemeinschaften (1973), S. 60, Tapon/Tong (1997), S. 22, Kaltwasser (1994), S. 138 f., Teece (1988), S. 261. 
sind, weitere FuE-Leistungen extern zu beziehen. Die im Zeitablauf steigende Abhängigkeit des Auftraggebers lässt sodann eine asymmetrische Beziehung mit starker Stellung des FuE-Dienstleisters und der resultiertenden Hold-upGefahr entstehen. Da sich in einer solchen Situation die Preisverhandlungen bei Nachfolgeaufträgen als schwierig erweisen können, ist ferner zu vermuten, dass im Endeffekt eine Übernahme des FuE-Dienstleisters, d.h. eine vertikale Integration beider zuvor selbstständiger Tauschpartner, aus Auftraggebersicht die ökonomisch sinnvollere Alternative darstellt.

\subsubsection{Zum Einfluss der Transaktionseigenschaften}

Der Bedingung der Faktorspezifität kommt insofern eine besondere Rolle zu, als im Vorfeld einer FuE-Vertragsbeziehung spezifische Investitionen in spezielle technische Ausrüstungen notwendig sein können oder nicht rückholbare Kosten im Zusammenhang mit der Einstellung spezialisierten Fachpersonals oder Durchführung von Machbarkeitsstudien anfallen können. ${ }^{318}$ Werden derartige transaktionsspezifische Investitionen getätigt, entstehen für den FuEDienstleister erhöhte fixe, zum großen Teil versunkene Kosten, die zu kurzund längerfristiger Abhängigkeit und Hold-up-Gefahr führen können. Da aus der Sicht des TKA die durch hochspezifische Investitionen gestützten Transaktionen die Entscheidung für eine vertikale Integration begünstigen, ist von der Wahl der Vertragsforschung als einer Marktlösung dann auszugehen, wenn keine hochspezifischen Transaktionen getätigt werden müssen. Dies ist dann gegeben, wenn der Auftragnehmer die erforderlichen Investitionen bereits in der Vergangenheit getätigt hat und/oder die geforderte Spezialkompetenz auf einem bestimmten Bereich aufweist, die nicht erst zeit- und kostenintensiv aufgebaut werden muss. ${ }^{319}$ Folglich kann vermutet werden, dass Auftragnehmer unter denjenigen Unternehmen zu erwarten sind, die über materielle (Ausrüstungsgegenstände) und immaterielle (distinktive Kompetenzen) Ressourcen verfügen, die nicht erst als transaktionsspezifische Investitionen aufgebracht werden müssen (Hypothese $\mathbf{3}$ ).

Häufig anfallende Prozesse sollen aus der transaktionskostenorientierten Sicht intern durchgeführt werden, während für vereinzelt abzuwickelnde Vorgänge der Marktlösung der Vorzug gegeben wird. Forschung und Entwicklungstätigkeit als kontinuierlicher, kumulativer Produktionsvorgang soll folglich primär

\footnotetext{
318 Vgl. Rüdiger (2000), S. 102.

319 Vgl. Schneider/Zieringer (1991), S. 65.
} 
intern abgewickelt werden; Auftragsvergabe im FuE-Bereich kann kosteneinsparend in den zwei folgenden Situationen wirken. Aus Auftraggebersicht bietet sich zum einen die Möglichkeit an, auf das Instrument der Vertragsforschung dann zurückzugreifen, wenn einmalige Leistungen erforderlich werden, die im Rahmen größerer Projekte eingebettet sind. ${ }^{320}$ Zum anderen kann bei kurzfristig anfallenden Kapazitätsengpässen die Auftragsvergabe sinnvoll sein, wenn eine externe FuE-Dienstleistung die kostengünstigere Alternative zum zusätzlichen Aufbau der im Regelfall nicht auszulastenden Kapazitäten darstellt. ${ }^{321}$ Der komparative Vorteil der Marktlösung begründet sich in diesem Falle in der erhöhten Anpassungsflexibilität. Spiegelbildlich können die Auftragnehmer dann Vertragsforschungsdienstleistungen anbieten, wenn eine Grundauslastung mit eigenen FuE-Projekten kurzfristig nicht gegeben ist. Folglich kann festgehalten werden: die Vertragsforschung stellt ein kostengünstiges institutionelles Arrangement dar im Kontext der Auslastungsschwankungen und der Engpassüberwindung (Hypothese 4).

Hohe Unsicherheit begünstigt in der Williamsonschen Transaktionskostenökonomik die Entscheidung für eine interne Prozessabwicklung. Im Kontext der FuE-Tätigkeit entsteht Unsicherheit nicht nur aufgrund der auftretenden exogenen Umweltstörungen: Die Natur der FuE-Tätigkeit ist selbst maßgeblich für die Existenz immenser Unsicherheiten verantwortlich. Daher stellt die Auftragsvergabe aus der transaktionskostenorientierten Perspektive ein stark risikobehaftetes institutionelles Arrangement zur Abwicklung von Forschungstätigkeit dar; Entscheidungen für Vertragsforschung erscheinen nur dann ökonomisch sinnvoller, wenn es sich bei der zu erbringenden Leistung um eine routinierte Bearbeitung von gut spezifizierbaren FuE-Teilleistungen handelt. ${ }^{322}$

Entgegen den Postulaten der Transaktionskostentheorie wird in der Literatur jedoch häufig argumentiert, dass den unternehmensexternen Koordinationsformen gerade in einem durch Unsicherheiten gekennzeichneten Umfeld eine

320 Koschatzky et al. nennen ein Beispiel aus der Erhebungspraxis, das in dem vorliegenden Zusammenhang zur Verdeutlichung herangezogen werden kann: Die Beauftragung von einem Unternehmen der Informationstechnologie mit dem Aufbau einer Datenbank, die für biologische Forschungen in einem Pharma-Konzern genutzt werden kann, vgl. Koschatzky/Reinhard/Grenzmann (2003), S. 30.

${ }^{321}$ Vgl. Schneider/Zieringer (1991), S. 84, Kommission der Europäischen Gemeinschaften (1973), S. 44. In diesem Zusammenhang erwähnt auch Kaltwasser die Rolle der Vertragsforschung als eines Mittels zur Entlastung der unternehmensinternen FuE-Bereiche, vgl. Kaltwasser (1994), S. 166.

322 Vgl. Tapon/Thong (1997), S. 27. 
besondere Rolle zukomme. So dürfte es beispielweise den Unternehmen der Spitzentechnologie oft schwer fallen, die aktuellen technologischen Entwicklungen stets zu verfolgen, ihr Potenzial jeweils rechtzeitig valide einzuschätzen und in ein eigenes FuE-Programm zu integrieren. Pisano (1991) verweist in diesem Zusammenhang auf das Beispiel der pharmazeutischen Industrie. In der zweiten Hälfte der 70er Jahre haben nur einige wenige Pharma-Konzerne Forschung auf dem sich gerade in der Entstehungsphase befindenden Gebiet der Biotechnologie betrieben. Den Zugang zu den neuen technologischen Entwicklungen konnten sie sich jedoch sichern durch Nutzung unternehmensexterner Koordinationsformen wie Kapitalbeteiligungen an Biotechnologieunternehmen, Gründungen von research joint ventures und Rückgriff auf das Instrument der Vertragsforschung, ohne dass der Aufbau unternehmensinterner Kapazitäten im unsicheren Umfeld notwendig war. Diese Strategie der PharmaKonzerne führte zu einem erheblichen Bedeutungszugewinn der Vertragsforschung mit der Entstehung des FuE-Dienstleistungsmarktes mit spezialisierten Biotechnologieunternehmen auf der Angebotsseite. ${ }^{323}$ Auf ähnliche Weise wird auch in der Studie von Tapon und Thong argumentiert:

\begin{abstract}
"No firm alone can keep up under such conditions when the fields of science are in a complete state of flux. Scientific bets cannot be placed without more information. Outsourcing research, at this early and riskiest stage, is a costeffective way of exploring new technologies without having to commit resources and hiring researchers. “324
\end{abstract}

Das von Pisano angeführte Beispiel sowie die Überlegungen von Tapon und Tong lassen vermuten, dass Vertragsforschung für Unternehmen der Spitzentechnologie eine wichtige Rolle spielt als ein Instrument der Sondierung von neuen technologischen Entwicklungen (Hypothese 5). Schneider und Zieringer verweisen in ihrer Studie ebenfalls auf dieses Phänomen und bezeichnen die erläuterte Vorgehensweise der forschungsintensiven Unternehmen als „FuE-Window-Politik“325.

\title{
4.2.3.3 Zur Frage der Koordinationsfähigkeit
}

Schließlich soll die Problematik der Komplexität der hierarchischen Steuerung von FuE-Prozessen angesprochen werden. Williamson weist auf die Koordi-

$323 \mathrm{Vgl}$. Pisano (1991), S. 239 f.

324 Tapon/Tong (1997), S. 13.

$325 \mathrm{Vgl}$. Schneider/Zieringer (1991), S. 67. Zu dieser Bezeichnung vgl. auch Tapon/Tong (1997), S. 14. 
nationsschwierigkeiten der betriebsinternen Ressourcenallokation hin, welche aufgrund von Informationsvermittlungsproblemen in hierarchisch organisierten Unternehmenseinheiten entstehen. Die resultierenden Fehler bei der selektiven Intervention erhöhen die Wahrscheinlichkeit einer unproduktiveren Ressourcenzuweisung. Zieht man in diesem Zusammenhang zusätzlich die Überlegungen von Coase über die eingeschränkte Koordinationsfähigkeit eines Unternehmers bei zunehmender Komplexität der Verflechtungen im Rahmen einer großen Unternehmenseinheit in Betracht, dann kommt man zu dem Ergebnis, dass bei wachsender Unternehmensgröße die Fehlallokation von Ressourcen innerhalb einer hierarchischen Organisation zunehmen muss, was die Kosten interner Steuerung über die Kosten ansteigen läßt, die bei Bezugs einer Leistung über den Markt entstehen. Dies lässt die Schlussfolgerung zu, dass die Auftragsvergabe in der Gruppe der Großunternehmen als ein Instrument zur Bewältigung interner Koordinationsprobleme eingesetzt wird (Hypothese 6).

\subsubsection{Grenzen der transaktionskostenorientierten Ana- lyse}

Ziel der Analyse im vorangegangenen Abschnitt war es zu überprüfen, welche Argumentationsbausteine der Transaktionskostenansatz bei der Ableitung der Hypothesen über die Bestimmungsgründe der Vertragsforschung liefern kann. In den nachfolgenden Ausführungen wird daher nicht auf eine umfassende kritische Würdigung des TKA abgestellt. ${ }^{326}$ Der Schwerpunkt soll auf die Einschätzung der Eignung dieses Forschungsprogramms für die Erklärung der institutionellen Produktionsstruktur im FuE-Bereich gelegt werden, wobei insbesondere der Frage nach potenziellen Erklärungsdefiziten nachgegangen werden soll.

Bei der Würdigung der transaktionskostenorientierten Betrachtungsweise kann zunächst darauf verwiesen werden, dass es dieser facettenreichen verbalen Variante des institutionenökonomischen Forschungsprogramms gelingt, konzeptionelle Grundlagen für die Analyse von Transaktionsbeziehungen zu legen, die durch wechselseitige Abhängigkeit der Wirtschaftsakteure gekennzeichnet sind. Das Williamsonsche Gedankengut ermöglicht sodann die Untersuchung

326 Vgl. Terberger (1994), Alchian/Woodward (1988). Zur kritischen Auseinandersetzung mit dem Transaktionskostenansatz vgl. Schneider (1985), Noorderhaven (1995), Slater/Spencer (2000), Windsperger (1997), Windsperger (1998), Ghoshal/Moran (1996). 
der Vertragsforschungsaktivitäten vor dem Hintergrund starker Interdependenzen zwischen den Wirtschaftsakteuren. ${ }^{327}$ Es werden Gefahren dieser Koordinationsform der FuE-Tätigkeit hervorgehoben, welche aufgrund der Vertragsunvollständigkeit in Verbindung mit der den Transaktionspartnern unterstellten Tendenz zum opportunistischen Verhalten entstehen. Durch den Verweis auf Schwierigkeiten bei der Vertragsfestlegung und -abwicklung kann in der Folge erklärt werden, warum die FuE-Tätigkeit schwerpunktmäßig intern abgewickelt wird. Die Analyse der Maßnahmen, die getroffen werden können, um die entstehenden Gefahren zu mildern, ermöglicht zugleich die Ableitung von Bedingungen, unter denen Vertragsforschung eine kostengünstige Alternative darstellt.

Ein wesentlicher Vorteil der transaktionskostenorientierten Analyse besteht ferner darin, dass dieses Forschungsprogramm hilft, das Spektrum der Koordinationsformen für FuE-Tätigkeit im Formenkontinuum zwischen Markt und Hierarchie anzusiedeln. Vertragsforschung kann dadurch als eine buyAlternative von anderen Organisationsformen abgegrenzt werden. Problematisch erscheint allerdings, dass Williamson die Wahl zwischen einer hierarchischen, durch eine zentrale Instanz gesteuerten Koordination der Ressourcenallokation im Rahmen einer Unternehmung und der Marktlösung fast ausschließlich unter dem Gesichtspunkt der Transaktionskosteneinsparung analysiert. ${ }^{328}$ Zwar betont er, dass eine umfassende Analyse der make-or-buyEntscheidungen der Interdependenzen zwischen der Produktions- und Transaktionssphäre Rechnung tragen muss. ${ }^{329}$ Allerdings wird bei der Untersuchung der Grundlage einzelwirtschaftlicher Entscheidungen für unternehmensinterne oder -externe Koordination die Annahme gemacht, dass die Kosten einer bestimmten Produktionsleistung gleich hoch anfallen, unabhängig davon, ob diese Leistung unternehmensintern erstellt oder unternehmensextern bezogen

\footnotetext{
327 Vgl. Martiensen (2000), S. 119.
}

$328 \mathrm{Vgl}$. dazu die Aussage von Williamson: „Die Transaktionskostenanalyse ersetzt die bislang vorherrschende Beschäftigung mit Technologie und mit Produktions- (oder Verteilungs)kosten im Gleichgewicht durch eine Untersuchung der komparativen Kosten von Planung, Modifizierung und Überwachung der Aufgabenerfüllung in alternativen Beherrschungs- und Überwachungssystemen [...].“, Williamson (1990), S. 1.

$329 \mathrm{Vgl}$. dazu die Aussage von Williamson: „The criterion for organizing commercial transactions is assumed to be the strictly instrumental one of cost economizing. Essentially this takes two parts: economizing on production expense and economizing on transaction costs. In fact these are not independent and need to be adressed simultaneously. The study of the latter, however, is much less well developed and is emphasized here.", Williamson (1981), S. 1547. 
wird. Nur bei der Voraussetzung der identischen Produktionsbedingungen in jedem auf dem Markt agierenden Unternehmen kann die komparative Höhe der Transaktionskosten als das ausschlaggebende Entscheidungskriterium bei der Wahl der optimalen institutionellen Einbettung einer Transaktion fungieren. ${ }^{330}$ In Worten von Williamson:

„Ein sinnvolles Verfahren zur Erläuterung der Integrationsentscheidung besteht darin, die Technologie für die alternativen Organisationsformen konstant zu halten und offensichtliche Ursachen ökonomischer Nutzendifferentiale auszuschalten $[\ldots]$. “331

Mit der Annahme der homogenen Verteilung der Technologie zwischen den Wirtschaftsakteuren bzw. mit der Ausschaltung der „offensichtlichen Ursachen ökonomischer Nutzendifferenziale" wird in der Williamsonschen Transaktionskostenökonomik jedoch unterstellt, dass alle Unternehmen vollkommene Substitute zueinander sind. ${ }^{332}$ Dadurch werden Unternehmen als Beherrschungsund Überwachungsstrukturen angesehen, die im Produktionsbereich letztlich genauso wie im neoklassischen Marktmodell als homogene „Produktionsfunktionen" fungieren. ${ }^{333}$ Es überrascht folglich nicht, dass Williamson vorgeworfen wird, dass er in seiner Theorie das neoklassische Diktat der technologischen Produktionseffizienz durch den Determinismus des Kostenvergleichs in der Transaktionssphäre ersetzt. ${ }^{334}$ Diesem Vorwurf kann Williamson entgegenhalten, dass es sich hierbei um „eine Art Wiedergutmachung - angesichts der bisherigen Vernachlässigung und Unterschätzung“335 bzw. um eine „pädagogische Maßnahme" ${ }^{\text {336 }}$ handele.

Im Rahmen des Transaktionskostenansatzes kommt es folglich zur Überbetonung der Transaktions- gegenüber der Produktionssphäre. Weder die Ursachen der potenziell heterogenen Unternehmensleistung im Produktionsbereich noch ihre Bedeutung für Organisationsentscheidungen werden analysiert. Diese Aspekte können jedoch im Kontext der Vertragsforschung nicht unberücksichtigt bleiben insbesondere vor dem Hintergrund, dass sich der Zugriff auf unternehmensexterne Ressourcen in der bisherigen empirischen Forschung als eines

330 Vgl. Madhok (2002), S. 538.

331 Williamson (1990), S. 99.

332 Vgl. Pfaffmann (1999), S. 617, Chung (1998), S. 135, Foray (1991), S. 396.

333 Vgl. zu der Ansicht Pfaffmann (1999), S. 616, Englander (1988), S. 350.

334 Vgl. hierzu Englander (1988), S. 339.

335 Williamson (1990), S. 19.

336 Williamson (1988), S. 355. 
der zentralen Motive für die Auftragsvergabe herausstellt. Zur Erläuterung der Problematik der Heterogenität der Ressourcenausstattung muss deshalb auf alternative Ansätze zurückgegriffen werden. Dies soll im nächsten Abschnitt erfolgen. Es wird gezeigt, dass die Hartsche Modellierung des Property-RightsAnsatzes das Augenmerk auf die Bedeutung der zugrunde liegenden Ressourcenverteilung zwischen den Wirtschaftsakteuren lenkt und somit die Analyse der Organisationsentscheidungen vor dem Hintergrund der Ressourcenbasis der Wirtschaftsakteure ermöglicht.

Es kann schließlich darauf hingewiesen werden, dass die in dieser Arbeit verfolgte Vorgehensweise, die die Beleuchtung der Ressourcenallokation im FuEBereich aus verschiedenen theoretischen Perspektiven vornimmt, der von Williamson ausdrücklich empfohlenen Methodik folgt. Williamson stellt hierzu fest:

„In Anbetracht der Komplexität der betrachteten Erscheinungen sollte die Transaktionskostentheorie oft zusätzlich zu alternativen Analyseverfahren verwendet werden, durchaus nicht diese ausschließen." ${ }^{337}$

\subsection{Der Property-Rights-Ansatz}

Der Property-Rights-Ansatz (PRA) geht auf Grunderkenntnisse von Coase zurück $^{338}$, und wurde insbesondere von Hart, Grossman und Moore weiterentwickelt. ${ }^{339} \mathrm{Da}$ dieser Ansatz auf den Williamsonschen Vorüberlegungen zur Bedeutung von Vertragsunvollständigkeit und Faktorspezifität aufbaut, wird zur Beschreibung dieser Forschungsrichtung bzw. der auf Grossman und Hart zurückgehenden Modellierung dieses Forschungsansatzes, auch die Bezeichnung „Theorie der unvollständigen Verträge“ verwendet. ${ }^{340}$

Nachfolgend soll untersucht werden, wie sich aus der Perspektive des PropertyRights-Ansatzes Integrations- und Desintegrationsentscheidungen der Wirtschaftsakteure erklären lassen. Hierzu werden zunächst in Abschnitt 4.3.1 kurz die für die Hartsche Modellbildung grundlegenden Definitionen des Eigentums

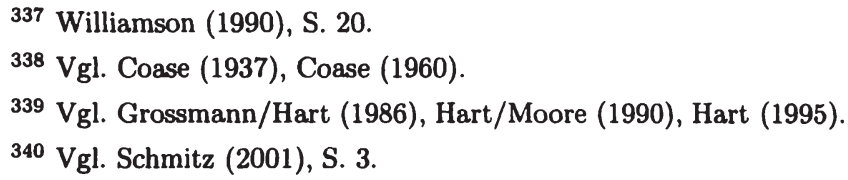


und der Unternehmung erläutert. Anschließend erfolgt in Abschnitt 4.3.2 eine verbale Erläuterung der modellierten Situation und in Abschnitt 4.3 .3 eine Darstellung des Modells. Bei der Vorstellung des Modells wird auf eine vollständige Darlegung aller Annahmen und Beweise verzichtet ${ }^{341}$; es sollen nur die für das Verständnis des Modells notwendigen Bausteine erläutert werden.

Auf der Grundlage der Modellanalyse wird in Abschnitt 4.3.4 diskutiert, welchen Beitrag der Property-Rights-Ansatz bei der Erklärung des Phänomens der Vertragsforschung liefern kann.

\subsubsection{Definitorische Grundlagen}

Grundlegend für die Hartsche Modellbildung ist zunächst die Definition des Eigentums. Eigentum definiert Hart als Kontrolle über die Verwendungsmöglichkeiten der physischen Vermögensteile einer Unternehmung. ${ }^{342}$ In einer Welt unvollständiger Verträge spielt diese Verfügungsmacht eine herausragende Rolle: "ownership is a source of power when contracts are incomplete“343. Können im Zuge einer Vertragsbeziehung nicht alle Umweltzustände antizipiert und in der Folge das Verhalten der Akteure nicht vollständig vertraglich festgelegt werden, dann bleiben allein in den Händen des Eigentümers die sogenannten Residualrechte: die Entscheidungsmacht über die Verwendung eigener Ressourcen in ex-ante nicht vorgesehenen Situationen. In Vertragsbeziehungen, die durch wechselseitige Abhängigkeitsverhältnisse aufgrund von spezifischen Investitionen gekennzeichnet sind, bedeutet die Beibehaltung der Residualrechte die Entstehung der Hold-up-Gefahr. Derjenige, der die Kontrollrechte behält, genießt den Vorteil einer stärkeren Verhandlungsposition bei im Bedarfsfall anfallenden Nachverhandlungen. Da er in diesem Falle einen höheren Anteil am Ertrag aus einer Transaktionsbeziehung antizipieren kann, hat dies einen Einfluss auf seine Investitionsentscheidungen im Bereich der transaktionsspezifischen Investitionen. ${ }^{344}$

In der Folge wird ein Unternehmen im Property-Rights-Ansatz als Bündel von Vermögensteilen (collection of physical assets ${ }^{345}$ ) im einheitlichen Eigentum

341 Vgl. hierzu Hart (1995), S. 34 ff., Siemer (2004), S. 33 ff.

342 Vgl. dazu Hart (1995), S. 30, Hart (1989), S. 1765.

${ }^{343}$ Hart (1995), S. 29.

344 Vgl. Tirole (1999), S. 749, Schmitz (2001), S. 11, Gibbons (2005), S. 205.

345 Vgl. Moore (1992), S. 496. 
konzeptualisiert ${ }^{346}$; bei der vertikalen Integration kommt es zur Überführung aller physischen Vermögensteile in die Hand eines einzigen Eigentümers. ${ }^{347}$ Physische Vermögenswerte (Ressourcen, Vermögensteile) umfassen Maschinen, Lagerbestände, Gebäude, Grundstücke, Patente, Kundenlisten etc. Das Humankapital hingegen bleibt vor und nach der Integration im Eigentum der Angestellten. ${ }^{348}$

\subsubsection{Die zu modellierende Situation}

Hart knüpft in seiner Analyse an die von Williamson eingeführten Konzepte der Unvollständigkeit von Verträgen und der durch wechselseitige Abhängigkeitsverhältnisse gekennzeichneten Transaktionsbeziehungen an und untersucht in seinem Modell die folgende Marktsituation. Zwei Unternehmen (Zulieferer und Endprodukthersteller) möchten in eine Transaktionsbeziehung treten, für die beiderseits transaktionspezifische Vorlaufinvestitionen notwendig sind. Die Höhe der Investitionen wird von beiden Vertragspartnern autonom entschieden, da sie ex-ante vertraglich nicht festgelegt werden kann.

Zwei Fragestellungen stehen im Mittelpunkt der Hartschen Analyse. Zum einen wird untersucht, wie sich die im Vorfeld einer abzuwickelnden Transaktion vorliegenden Eigenstumsverhältnisse (d.h. die Art der Verteilung von Vermögensgegenständen zwischen den Akteuren) auf die Entscheidungen über die Höhe der zu tätigenden Investitionen auswirken. Zu den untersuchten Eigentumsverhältnissen gehören 1) vertikale Integration vom Typ 1, in der der Endprodukthersteller den Zulieferer übernimmt, 2) vertikale Integration vom Typ 2, in der der Zulieferer den Endprodukthersteller aufkauft und 3) Selbstständigkeit der Transaktionspartner. ${ }^{349}$ Im Falle von Integrationslösungen vom Typ 1 und Typ 2 verfügt eine Unternehmung über Kontrollrechte über alle Ressourcen $\left(a_{1}, a_{2}\right)$; bei Selbstständigkeit behalten die jeweiligen Transaktionspartner residuelle Kontrollrechte über den Einsatz eigener Vermögensgegenstände. Eine schematische Darstellung der Zuordnung der Vermögensgegenstände zu den einzelnen Eigentumsstrukturen kann der Tabelle 4.2 entnommen werden.

346 Vgl. Hart (1989), S. 1771.

347 Vgl. Schweizer (1999), S. 7.

348 Vgl. Hart (1995), S. 29, Moore (1992), S. 496.

${ }^{349}$ Es wird angenommen, dass beide (risikoneutrale) Vertragspartner durch Finanzierungsprobleme in ihren Entscheidungen nicht eingeschränkt sind, vgl. Hart (1995), S. 35. 
Tabelle 4.2: Eigenstumsstrukturen (ownership structures) im Hartschen Modell

keine Integration Integrationstyp 1 Integrationstyp 2

\begin{tabular}{lccc}
\hline \hline $\begin{array}{l}\text { Endprodukt } \\
\text { hersteller (A) }\end{array}$ & $A=\left\{a_{1}\right\}$ & $A=\left\{a_{1}, a_{2}\right\}$ & $A=\emptyset$ \\
\hline Zulieferer (B) & $B=\left\{a_{2}\right\}$ & $B=\emptyset$ & $B=\left\{a_{1}, a_{2}\right\}$ \\
\hline
\end{tabular}

Quelle: Eigene Darstellung.

Zum anderen geht Hart der Frage nach der relativen Vorteilhaftigkeit einer Integrations- gegenüber einer Marktlösung in Abhängigkeit von der Art und Konstellation der den beiden Partnern zur Verfügung stehenden Ressourcen nach. Diese Frage erscheint vor dem Hintergrund der Zielsetzung der vorliegenden Arbeit besonders interessant: Auf der Basis des Hartschen Modells können Bedingungen identifiziert werden, welche Entscheidungen für Markttransaktionen und gegen eine vertikale Integration beider Partner begünstigen.

Es erscheint sinnvoll, die von Hart modellierte Situation an einem Beispiel zu verdeutlichen, anhand dessen sich der zu erläuternde Modellrahmen auf den Kontext der Vertragsforschung übertragen lässt. Hierbei kann auf die in der Literatur mehrmals erörterte Problematik der Organisation der FuE-Tätigkeit in der Pharmaindustrie zurückgegriffen werden. ${ }^{350}$ Betrachtet werden zwei Unternehmen, ein Pharma-Konzern (Endprodukthersteller, A) und ein Biotechnologieunternehmen (Zulieferer, B). Aus der Perspektive des Pharma-Konzerns stellt sich die Frage, ob die Entwicklungsleistungen des Biotechnologieunternehmens im Rahmen einer Vertragsforschungsbeziehung vergütet werden sollten (d.h. ob eine Markttransaktion zwischen zwei unabhängigen Unternehmen stattfindet), oder ob die Übernahme des Biotechnologieunternehmens die ökonomisch sinnvollere Alternative darstellt. Im letzteren Fall kommt es - in Terminologie des Hartschen Modells - zur vertikalen Integration vom Typ 1.

Im Modell wird vorausgesetzt, dass eine zwischen beiden Parteien antizipierte Transaktion beiderseits Vorlaufinvestitionen erfordert, wie es zum Beispiel folgender Situation entspräche: Der Zulieferer (Biotechnologieunternehmen) konzentriert sich auf die Weiterentwicklung eines gentechnischen/diagnostischen

350 Vgl. hierzu insbesondere Pisano (1991). 
Verfahrens. ${ }^{351}$ Der Endprodukthersteller - die Firma der pharmazeutischen Industrie - unternimmt dagegen (wie von Hart explizit vorgesehen ${ }^{352}$ ) Investitionen, die mit der Vorbereitung der Markteinführung verbunden sind.

Eine derartige „vertikale Arbeitsteilung“ (vertical division of labor) zwischen Biotechnologieunternehmen und etablierten Unternehmen der PharmaIndustrie im Bereich der Produktentwicklung beschreibt Pisano (1991) in seiner Studie über die Organisationsstruktur des Biotechnologiesektors. Er sammelt Daten zu neuen gentechnischen Arzneimitteln, die in den 80er Jahren von der Arzneimittelzulassungsbehörde der Vereinigten Staaten (Food and Drug Administration) zugelassen wurden bzw. für die Ende der 80er und Anfang der 90er Jahre eine Zulassung beantragt wurde und zeigt, dass die Mehrheit dieser Produkte von den Biotechnologieunternehmen entwickelt wurde, aber von den etablierten Pharma-Unternehmen vermarktet werden sollte. Diese Art der Arbeitsteilung führte er auf die komparativen Vorteile der etablierten Firmen im Bereich der Vermarktung von fertigen Produkten zurück. ${ }^{353}$

Der Ablauf der modellierten Transaktionsbeziehung kann anhand der schematischen Darstellung in Abb. 4.1 erläutert werden. Am Anfang der Transaktionsbeziehung $\left(t_{0}\right)$ einigen sich die Wirtschaftsakteure zunächst auf eine bestimmte Eigentumsordnung: Entweder bleiben die Unternehmen selbstständig oder es kommt zur vertikalen Integration. Aufgrund der Annahme der NichtVerifizierbarkeit von Teilen der Vertragsmodalitäten können sie weder den für den Erfolg der Forschungsanstrengungen benötigten finanziellen Aufwand (d.h. die Höhe der transaktionsspezifischen Investitionen) noch die Tauschmodalitäten ex-ante im Rahmen eines vollständigen Vertrages festlegen und sind daher auf Nachverhandlungen zum späteren Zeitpunkt angewiesen. Die Annahme der "Nicht-Verifizierbarkeit" bedeutet in diesem Zusammenhang, dass die Parteien nicht in der Lage sind, einen Vertrag zu formulieren, der sowohl die Beschaffenheit des zu übertragenden Transaktionsobjektes als auch die Höhe der notwendigen transaktionsspezifischen Investitionen so eindeutig festlegt, dass im Konfliktfall eine Übeprüfung durch eine dritte Partei (Gericht) unproblematisch erfolgen kann. ${ }^{354}$

351 In dem Modell wird vorausgesetzt, dass die transaktionsspezifische Investitionen des Zulieferers Einfluss auf seine Kostenfunktion hat, vgl. Hart (1995), S. 36.

352 Vgl. Hart (1995), S. 35.

$353 \mathrm{Vgl}$. Pisano (1991), S. $241 \mathrm{f}$.

354 Vgl. Hart (1995), S. 81. Für weitere Begründungen für die Annahme der Unvollständigkeit von Verträgen im Rahmen des PRA vgl. Siemer (2004), S. 91 ff. sowie die dort zitierte Literatur. 
Abbildung 4.1: Ablauf der Transaktionsbeziehung im Hartschen Modell

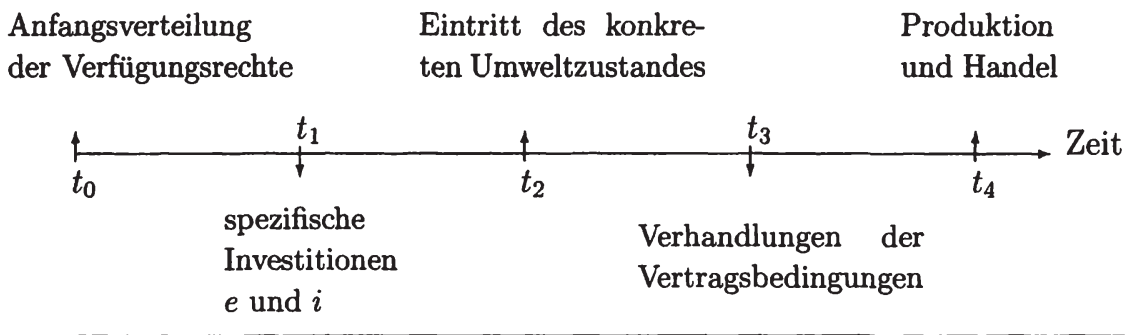

Quelle: Eigene Darstellung in Anlehnung an Erlei/Leschke/Sauerland (1999), S. 195.

Zum Zeitpunkt $t_{1}$ fällen beide Partner autonom Entscheidungen über die Höhe ihrer transaktionsspezifischen Investitionen und beobachten zum Zeitpunkt $t_{2}$ den eingetretenen Umweltzustand (wie beispielweise die Qualität des vom Biotechnologieunternehmen erarbeiteten Verfahrens oder die Wettbewerbssituation auf dem Absatzmarkt). Zum Zeitpunkt $t_{3}$ können die Vertragsmodalitäten festgelegt und im Zeitpunkt $t_{4}$ die antizipierte Transaktionsbeziehung vollzogen werden.

\subsubsection{Modellanalyse}

Nachdem die Marktsituation beschrieben wurde, erfolgt nun die Erläuterung des Hartschen Modells.

\subsubsection{Modellannahmen}

Wie bereits erläutert, beschreibt das Modell von Hart eine bilaterale Beziehung zwischen einem Endprodukthersteller (A) und einem Zulieferer (B), die transaktionsspezifische Investitionen ( $i$ bzw. $e$ ) auf beiden Seiten erfordert. Die von den beiden Transaktionspartnern getätigten Vorlaufinvestitionen beeinflussen die Ertrags- bzw. Kostenfunktion der Unternehmen, wobei der gesamte Ex-post-Payoff ${ }^{355}$ als Differenz zwischen den beiden Größen verstanden

355 Wie bei Siemer (2004) wird nachfolgend auf eine Übersetzung des englischen Begriffs "Payoff" verzichtet. 
wird. Können sich die beiden Unternehmen in der Vertragsabwicklungsphase auf einen Preis $p$ einigen, so wird die Transaktionsbeziehung sowohl durch die Verfügbarkeit aller physischen Vermögensgegenstände als auch des Humankapitals beider Parteien gestützt. Der gesamte Ex-post-Payoff ist dann gegeben durch:

$$
[R(i)-p]+[p-C(e)]=R(i)-C(e)
$$

Beim Scheitern der Nachverhandlungen müssen sie auf den freien Markt ausweichen und den gegebenen Preis von $\bar{p}$ akzeptieren. In dieser Situation gewinnt auch die Eigentumsstruktur an Bedeutung. Der Ex-post-Payoff ist dann gegeben durch:

$$
[r(i, A)-\bar{p}]+[\bar{p}-c(e, B)]=r(i, A)-c(e, B)
$$

wobei $A \cap B=\emptyset$ und $A \cup B=\{a 1, a 2\}$ (wie in Tab. 4.2 dargestellt).

Im Vergleich zu der ersten Situation fällt der Ex-post-Payoff bei fehlender Einigung infolge der Annahme, dass die spezifischen Investitionen zumindest partiell humankapitalspezifisch sind, geringer aus, bleibt jedoch stets positiv:

$$
R(i)-C(e)>r(i, A)-c(e, B) \geq 0 \quad \forall i, e, A, B
$$

Schließlich wird angenommen, dass der Grenzertrag der transaktionsspezifischen Investitionen ( $e$ bzw. $i$ ) umso höher ist, je mehr Vermögensgegenstände der jeweiligen Partei zur Verfügung stehen:

$$
R^{\prime}(i)>r^{\prime}\left(i, a_{1}, a_{2}\right) \geq r^{\prime}\left(i, a_{1}\right) \geq r^{\prime}(i, \emptyset)
$$

für alle $0<i<\infty$ und

$$
\left|C^{\prime}(e)\right|>\left|c^{\natural}\left(e, a_{1}, a_{2}\right)\right| \geq c^{\natural}\left(e, a_{2}\right) \geq c^{\prime}(e, \emptyset) \mid
$$

für alle $0<e<\infty$.

\subsubsection{Aufteilung der Ex-post-Transaktionsrente}

Gilt die Annahme 4.3, so sind sich beide Parteien der Tatsache bewusst, dass sie im Vergleich zu der Situation, in der sie auf andere Marktteilnehmer zurückgreifen müssen, vorteilhafter dastehen, wenn sie sich über Vertragsmodalitäten mit dem antizipierten Transaktionspartner einigen, da in diesem Falle eine abzuschöpfende Rente in der Höhe von $[(R-C)-(r-c)]$ entsteht. Daher führen die Verhandlungen über die Vertragsmodalitäten (zum Zeitpunkt $t_{3}$, vgl. Abb. 
4.1) immer zu einer effizienten Ex-post-Lösung (efficient ex-post outcome); der implizit drohende Bezug einer nichtspezifischen Leistung über den freien Markt wird nicht erfolgen. ${ }^{356}$ Hart setzt voraus, dass diese Rente im Fall einer Einigung im 1:1 Verhältnis geteilt wird (Nash-Verhandlungslösung) ${ }^{357}$ Ex-post erhalten dann die Transaktionspartner:

$\Pi_{A}=R-p=r-\bar{p}+1 / 2[(R-C)-(r-c)]=-\bar{p}+1 / 2 R+1 / 2 r-1 / 2 C+1 / 2 c$

und

$$
\Pi_{B}=p-C=\bar{p}-c+1 / 2[(R-C)-(r-c)]=\bar{p}-1 / 2 C-1 / 2 c+1 / 2 R-1 / 2 r
$$

Der dazugehörende Preis ist gegeben durch:

$$
p=\bar{p}+1 / 2(R-r)-1 / 2(c-C)
$$

\subsubsection{Investitionsentscheidungen}

Das erste Ziel des Hartschen Modells ist es, die Auswirkungen der einzelnen Eigentumsstrukturen auf die Investitionsbereitschaft der Wirtschaftsakteure im Bereich der transaktionsspezifischen Investitionen zu untersuchen. Hierzu werden zunächst die Payoff-Funktionen der Unternehmen (wie in den Gleichungen 4.6 und 4.7) abzüglich der Investitionskosten ( $e$ und $i$ ) für vier folgende Fälle aufgestellt. Im 1. Fall koordinieren zwei selbstständige Unternehmen ihre Investitionsentscheidungen und maximieren den gesamten Ex-ante-Payoff $R(i)-i-C(e)-e$. Die unter den Annahmen des Modells einzige Lösung des Maximierungsproblems wird mit $\left(i^{*}, e^{*}\right)$ bezeichnet. Die Bedingungen erster Ordnung für gewinnmaximale Investitionsniveaus lauten:

$$
\begin{gathered}
R^{\prime}\left(i^{*}\right)=1 \\
\left|C^{\prime}\left(e^{*}\right)\right|=1
\end{gathered}
$$

In den weiteren drei Fällen werden die Investitionsentscheidungen autonom von einzelnen Parteien getroffen, wobei jeweils eine andere Eigentumsstruktur zugrunde liegt (Selbstständigkeit der Unternehmen, Übernahme des Zulieferers durch den Endprodukthersteller und Übernahme des Endproduktherstellers durch den Zulieferer). Die Bedingungen erster Ordnung lauten entsprechend:

356 Vgl. Hart (1995), S. 39, Siemer (2004), S. 37.

357 Vgl. Hart (1995), S. 39. 


$$
\begin{gathered}
1 / 2 R^{\prime}(i)+1 / 2 r^{`}(i, A)=1 \\
1 / 2\left|C^{\prime}(e)\right|+1 / 2\left|c^{\prime}(e, B)\right|=1
\end{gathered}
$$

Für die drei analysierten Eigentumsstrukturen ergibt sich (die Subskripte bei transaktionsspezifischen Investitionen stehen jeweils für die betrachtete Eigentumsstruktur):

a) bei Selbstständigkeit der Akteure (0):

$$
\begin{gathered}
1 / 2 R^{\natural}\left(i_{0}\right)+1 / 2 r^{`}\left(i_{0}, a_{1}\right)=1 \\
1 / 2\left|C^{`}\left(e_{0}\right)\right|+1 / 2\left|c^{\natural}\left(e_{0}, a_{2}\right)\right|=1
\end{gathered}
$$

b) bei der Übernahme des Zulieferers durch den Endprodukthersteller (Integrationstyp 1):

$$
\begin{gathered}
1 / 2 R^{\prime}\left(i_{1}\right)+1 / 2 r^{\prime}\left(i_{1}, a_{1}, a_{2}\right)=1 \\
1 / 2\left|C^{\prime}\left(e_{1}\right)\right|+1 / 2\left|c^{\prime}\left(e_{1}, \emptyset\right)\right|=1
\end{gathered}
$$

c) bei der Übernahme des Endproduktherstellers durch den Zulieferer (Integrationstyp 2):

$$
\begin{gathered}
1 / 2 R^{\prime}\left(i_{2}\right)+1 / 2 r^{\prime}\left(i_{2}, \emptyset\right)=1 \\
1 / 2\left|C^{\prime}\left(e_{2}\right)\right|+1 / 2\left|c^{\prime}\left(e_{2}, a_{1}, a_{2}\right)\right|=1
\end{gathered}
$$

Erfüllen $i$ und $e$ die Bedingungen 4.11 und 4.12, dann gilt aufgrund der Annahmen 4.4 und 4.5, dass unter jeder Eigentumsordnung die Höhe der Investitionen suboptimal gegenüber der first-best-Lösung $\left(e^{*}, i^{*}\right)$ ausfällt (Satz 1). ${ }^{358}$ Insgesamt ergibt sich dann folgender Vergleich der unter jeder Eigentumsordnung getätigten Investitionen:

$$
\begin{gathered}
i^{*}>i_{1} \geq i_{0} \geq i_{2}, \\
e^{*}>e_{2} \geq e_{0} \geq e_{1} .
\end{gathered}
$$

Intuitiv kann das Ergebnis des suboptimalen Investitionsniveaus folgendermaBen begründet werden. Steigert der Endprodukthersteller die transaktionsspezifischen Investitionen, so erhöht sich der Ertrag um $R^{\prime}(i)$. Er profitiert davon jedoch nur in der Höhe von $1 / 2 R^{\prime}(i)+1 / 2 r^{\prime}(i, A)<R^{\prime}(i)$; der Rest kommt dem Zulieferer zugute. Als eigennütziger Wirtschaftsakteur berücksichtigt der Endprodukthersteller den Payoff-Zuwachs beim Zulieferer nicht. Seine Anreize zur Ausdehnung des Investitionsniveaus sinken. Die gleiche Überlegung gilt für die Entscheidungssituation des Zulieferers. ${ }^{359}$

358 Für den Beweis vgl. Hart (1995), S. 41.

359 Vgl. Hart (1990), S. 41. 


\subsubsection{Analyse der optimalen Eigentumsstruktur}

Im Rahmen seines Modells gelingt es Hart nicht nur, die Existenz der exante Vertragskosten in der Form der Verzerrung der Investitionsbereitschaft zu zeigen. Hart untersucht auch, wie sich das im Vorfeld der abzuwickelnden Transaktion geltende Ressourcenverteilungsmuster zwischen den beiden Unternehmen auf die relative Vorteilhaftigkeit einzelner Eigentumsstrukturen auswirkt. Er untersucht folgende Möglichkeiten: unterschiedliche Elastizitäten bzw. Produktivitäten der Investitionen, Unabhängigkeit bzw. Komplementarität der Produktionsfaktoren und Unverzichtbarkeit des Humankapitals. Für die Fragestellung der vorliegenden Arbeit erscheinen insbesondere die letzten drei der genannten Szenarien interessant. ${ }^{360}$

Bei Unabhängigkeit der Ressourcen beider Unternehmen steigt laut Definition die Grenzproduktivität der spezifischen Investition eines Partners nicht, wenn er zusätzlich die Kontrollrechte an den Produktionsfaktoren des anderen Partners übernimmt, d.h. es gilt $r^{\natural}\left(i, a_{1}, a_{2}\right) \equiv r^{\natural}\left(i, a_{1}\right)$ und $c^{\natural}\left(e, a_{1}, a_{2}\right) \equiv c^{c}\left(e, a_{2}\right)$. In diesem Falle sind sowohl die Gleichungen 4.13 und 4.15 als auch 4.14 und 4.18 identisch, d.h. $i_{1}=i_{0}$ und $e_{2}=e_{0}$. Da jedoch $e_{1} \leq e_{0}$ und $i_{2} \leq i_{0}$, dominiert Nichtintegration beide Integrationstypen. Mit anderen Worten: Bei Unabhängigkeit der Ressourcen stellen Markttransaktionen zwischen den selbstständigen Unternehmen das optimale institutionelle Arrangement dar.

Komplementärität der Produktionsfaktoren liegt vor, wenn entweder $r^{\prime}\left(i, a_{1}\right)=r^{\prime}(i, \emptyset)$ oder $c^{\prime}\left(e, a_{2}\right)=c^{\prime}(e, \emptyset)$. Dies bedeutet, dass die Kontrolle allein über $a_{1}$ keine Auswirkungen auf die Grenzproduktivität der Investition des Endproduktherstellers bzw. die Kontrolle allein über $a_{2}$ keine Auswirkungen auf die Grenzproduktivität der Investition des Zulieferers hat. ${ }^{361}$ Anders ausgedrückt: Streng komplementäre Produktionsfaktoren erhöhen nur dann die Grenzproduktivität einer Investition, wenn sie gemeinsam in der Hand eines Investors liegen. ${ }^{362}$ In diesem Falle sind die Lösungen der Gleichungen 4.13 und 4.17 identisch, $i_{0}=i_{2}$. Da aber $e_{0} \leq e_{2}$, dominiert Integrationstyp 2 die Nichtintegration. Eine analoge Beweisführung zeigt, dass die Selbstständigkeit beider Akteure vom Integrationstyp 1 dominiert wird, wenn $c^{\natural}\left(e, a_{2}\right)=c^{\natural}(e, \emptyset)$. Bei strikter Komplementarität der Ressourcen stellt in der Folge die Integration eine optimale Lösung dar.

360 Für die Erläuterung der ersten beiden Szenarien vgl. Hart (1995), S. 44 ff.

361 Vgl. Hart (1995), S. 45.

362 Vgl. Siemer (2004), S. 41. 
Das Humankapital des Endproduktherstellers bzw. des Zulieferers ist unverzichtbar, wenn die transaktionsspezifischen Investitionsgrenzerträge unabhängig sind von der Verteilung der Kontrollrechte an den Produktionsfaktoren, d.h. $r^{\prime}\left(i, a_{1}, a_{2}\right) \equiv r^{\natural}(i, \emptyset)$ bzw. $c^{\prime}\left(e, a_{1}, a_{2}\right) \equiv c^{\natural}(e, \emptyset)$. Ist das Humankapital beider Parteien unverzichtbar, dann sind die Lösungen von $4.13,4.15,4.17$ sowie von $4.14,4.16$ und 4.18 identisch, d.h. $i_{0}=i_{1}=i_{2}$ und $e_{0}=e_{1}=e_{2}$. In dieser Situation ist die Integrationsform irrelevant. Ist das Humankapital nur einer Partei unverzichtbar, dann soll diese Partei die physischen Ressourcen der anderen Partei übernehmen. Bei Unverzichtbarkeit des Humankapitals des Endproduktherstellers sind die Lösungen der Gleichungen 4.14, 4.16 und 4.18 identisch, d.h. $e_{0}=e_{1}=e_{2}$. Da jedoch $i_{1} \geq i_{0} \geq i_{2}$, ist Integrationstyp 1 optimal. Eine analoge Beweisführung zeigt die Überlegenheit des Integrationstypus 2 bei Unverzichtbarkeit des Humankapitals des Zulieferers. ${ }^{363}$

\subsubsection{Implikationen im Kontext der Vertragsforschung}

\subsubsection{Zur Bedeutung der Vertragsunvollständigkeit}

Bei der Interpretation der Vertragsforschung aus der Perspektive des PropertyRights-Ansatzes kann zunächst darauf hingewiesen werden, dass Transaktionsbeziehungen im PRA - genauso wie im Transaktionskostenansatz - vor dem Hintergrund der Unvollständigkeit von Verträgen analysiert werden. Während jedoch im TKA die Entstehung der Vertragsunvollständigkeit auf die Annahme der begrenzten Rationalität der Wirtschaftsakteure zurückgeführt werden kann, liegt die Quelle der Vertragsunvollständigkeit im PRA bei der Annahme der "Nicht-Verifizierbarkeit" von Teilen der Vertragsmodalitäten. ${ }^{364}$

Der Kondition der "Nicht-Verifizierbarkeit“ von Teilen der Vertragsmodalitäten kann im Umfeld der Vertragsforschungsbeziehungen eine besondere Rolle zukommen. Diesen Aspekt betont insbesondere eine im Auftrag der Kommission der Europäischen Gemeinschaften durchgeführte Studie, im Rahmen welcher Gespräche mit ausgewählten auftraggebenden und auftragnehmenden

363 Zur Diskussion der Annahmen und Ergebnisse der auf Grossman, Hart und Moore zurückgehenden Modellierung des Property-Rights-Ansatzes vgl. de Meza/Lockwood (1998), Chiu (1998), Edlin/Reichelstein (1996), Maskin/Tirole (1999); für eine Übersicht der Kritikpunkte vgl. Siemer (2004), S. 88-99. Eine Antwort auf einige Kritikpunkte bieten Hart (1995) sowie Hart und Moore (1999).

364 Vgl. Saussier (2000), S. 380. 
Unternehmen aus sechs europäischen Ländern ${ }^{365}$ durchgeführt wurden. In dieser Studie wird folgende aus der Sicht des Auftraggebers auftretende Problemsituation dargelegt: Bei der Festlegung des Forschungsprogramms darf der Auftraggeber „nicht zu enge Grenzen ziehen“366, da er mit der Beauftragung des unternehmensexternen Partners die Erwartung verbindet, dass der FuE-Dienstleister bei der ihm anvertrauten Aufgabe eigene Erfahrungen und spezifisches Know-how beisteuert, um zu einer neuartigen Problemlösung zu gelangen - und nicht nur das festgelegte Programm bearbeitet. Eine zu große Freiheit für den Auftragnehmer birgt jedoch die Gefahr in sich, dass der FuE-Dienstleister die entstandenen Freiräume nutzen kann, um eigenen angrenzenden - Interessengebieten nachzugehen, da der Auftraggeber nicht in der Lage ist einzuschätzen, inwieweit bestimmte Forschungsleistungen bei der Bearbeitung des gestellten Problems absolut notwendig sind. Diese Gefahren dürfen - in der Einschätzung dieser Studie - insbesondere für jene Unternehmen von Bedeutung sein, die nicht über eigene FuE-Kapazitäten verfügen und folglich größere Schwierigkeiten bei der Überwachung der FuE-Leistungen des Auftragnehmers haben. ${ }^{367}$ Daher dürfte für Unternehmen ohne eigene FuEKompetenz das Instrument der Vertragsforschung mit besonderen Gefahren verbunden sein und folglich die Möglichkeiten einer substitutiven - zur eigenen FuE-Tätigkeit - externen Wissensbeschaffung deutlich eingrenzen. Bei forschungstätigen und forschungsintensiven Unternehmen dürfte dagegen die Problematik der "Nicht-Verifizierbarkeit" der im Rahmen der Vertragsforschungsbeziehung zu bezahlenden Teilleistungen weniger ausgeprägt sein. Im Ergebnis kann die Hypothese formuliert werden, dass interne FuE-Kompetenz die Entscheidung für eine Auftragsvergabe begünstigt (Hypothese 7).

Die „Nicht-Verifizierbarkeit“ von Teilen der Vertragsmodalitäten stellt jedoch auch ein Problem im Zusammenhang mit der Übertragung von Resultaten der FuE-Tätigkeit dar. So stellt sich aus der Sicht des Auftraggebers die Frage, ob alle relevanten Ergebnisse an ihn weitergeleitet wurden oder ob der FuE-Dienstleister bewusst einen Teil seiner Erkenntnisse zurückhält, um sich auf diese Weise weitere Auftragsarbeiten zu sichern. ${ }^{368}$ Insbesondere dürften Wissenskomponenten mit Know-how-Charakter leicht dem Auftraggeber vorenthalten werden. Behält der Auftragnehmer die Residualrechte an dem erar-

${ }^{365}$ Italien, Frankreich, Bundesrepublik Deutschland, Belgien, Niederlande und Luxemburg, vgl. Kommission der Europäischen Gemeinschaften (1973), S. 5.

366 Kommission der Europäischen Gemeinschaften (1973), S. 56.

367 Vgl. Kommission der Europäischen Gemeinschaften (1973), S. 56 f.

$368 \mathrm{Vgl}$. Kommission der Europäischen Gemeinschaften (1973), S. 54. 
beiteten Wissen, dann entsteht eine besondere Gefahr für den Auftraggeber auch dadurch, dass das im Rahmen der bezahlten Forschungsleistung erzielte Know-how vom Dienstleister bei Vertragsforschungsbeziehungen mit weiteren Unternehmen verwertet und/oder weitergegeben wird. Inwieweit diese Gefahr tatsächlich zutrifft, dürfte aus der Sicht des Auftraggebers schwer zu ermitteln sein. Auch in diesem Zusammenhang kann auf die besondere Rolle des Vertrauensverhältnisses zwischen den Transaktionspartnern verwiesen werden (vgl. Hypothese 2).

\subsubsection{Ressourcen und Organisationsentscheidungen}

Der zentrale Vorteil des Property-Rights-Ansatzes begründet sich in der Hervorhebung der Bedeutung von Ressourcensituationen einzelner Unternehmen bei der Wahl einer allokationsoptimalen Eigentumsstruktur. Es kann gezeigt werden, dass bei bestimmten Konstellationen der Ressourcen die vertikale Integration der Selbstständigkeit von Wirtschaftsakteuren vorgezogen wird. Dies ist insbesondere dann der Fall, wenn die Ressourcen beider Transaktionspartner komplementär sind bzw. das Humankapital nur eines Partners unverzichtbar ist.

Das erste für die vorliegende Arbeit als zentral einzustufende Ergebnis der Hartschen Analyse lautet somit: Streng komplementäre Ressourcen sollen in einheitliches Eigentum überführt werden; bei Unabhängigkeit der Ressourcen stellen Markttransaktionen zwischen selbstständigen Akteuren ein optimales institutionelles Arrangement dar. Dieses Ergebnis kann herangezogen werden, um im Kontext der Desintegrationsentscheidungen (Ausgliederung von FuEGesellschaften ${ }^{369}$ ) den Unterschied zwischen einer transaktionskosten- und Property-Rights-orientierten Analyse zu verdeutlichen. Im Sinne der Hartschen Analyse ist davon auszugehen, dass komplementäre Ressourcen im einheitlichen Eigentum verbleiben; ausgegliedert werden periphäre Vermögensteile, d.h. diejenigen, die die Grenzerträge transaktionsspezifischer Investitionen nicht erhöhen. Aus der Perspektive des Endproduktherstellers stellt sich die Entscheidungssituation über die Ausgliederung unabhängiger Vermögensteile folgendermaßen dar: Sein Investitionsniveau bleibt gleich, unabhängig davon, ob er zusätzlich zu eigenen Ressourcen die Kontrolle über die (periphären)

369 In einer ifo-Befragung gaben $5 \%$ der innovierenden deutschen Unternehmen an, von 1997 bis 2000 eigenständige FuE-Tochterunternehmen ausgegründet zu haben, 7,3\% der Unternehmen planten eine Ausgliederung von FuE-Unternehmen im Zeitraum zwischen 2000 und 2003, vgl. Koschatzky/Reinhard/Grenzmann (2003), S. 69. 
Vermögensteile behält. Allerdings fällt das Investitionsniveau des Zulieferers (FuE-Dienstleisters) höher aus, wenn der Letztere über Residualrechte an eigenen Ressourcen verfügt. Daher kann es aus der Sicht des Endproduktherstellers sinnvoll sein, Teile eigener Ressourcen auszugliedern, da in diesem Falle der gesamte Ex-Post-Payoff höher ausfällt. Einschränkend gilt jedoch: Reine Ausgliederung ist nicht per se wirksam. Erst die Übertragung der Kontrollrechte an den Vermögensteilen eines ausgegliederten Unternehmens bewirkt die Umgestaltung der Anreizstruktur im Bereich der transaktionsspezifischen Investitionen bei der ausgegliederten Unternehmenseinheit.

Während folglich im Property-Rights-Ansatz eine Überführung von unabhängigen Vermögenswerten in eine FuE-Gesellschaft nur dann zur Reduzierung der ex-ante Kosten der Vertragsunvollständigkeit führt, wenn zugleich die Übertragung der Kontrollrechte an den der FuE-Gesellschaft zur Verfügung stehenden Ressourcen in getrenntes Eigentum erfolgt, kann aus der Perspektive des TKA eine Ausgliederung von nicht periphären Vermögensteilen effizient sein, wenn dadurch die in einer hierarchischen Organisation bestehenden Koordinationsprobleme bewältigt werden.

Treffen die Wirtschaftsakteure die Entscheidung, Vermögensteile auszugliedern - aufgrund interner Koordinationsprobleme oder in der Erwartung der Verbesserung der Leistungsanreize - dann kommt es zur Externalisierung von bisher intern abgewickelten Transaktionen. In der Folge kann im FuE-Bereich davon ausgegangen werden, dass Vertragsforschungsbeziehungen in der Folge von Ausgliederungsprozessen entstehen (Hypothese 8).

Das zweite zentrale Ergebnis der Hartschen Analyse lautet: Bei Unabdingbarkeit des Humankapitals beider Parteien ist die Eigentumsstruktur irrelevant; es kann sowohl zur vertikalen Integration als auch zu Markttransaktionen zwischen zwei selbstständigen Wirtschaftsakteuren kommen. Aus der Perspektive des Endproduktherstellers argumentiert heißt dies, dass die vertikale Integration mit dem Zulieferer nicht allokationseffizienter ist; es besteht kein Grund, den wirtschaftlich selbstständig agierenden FuE-Dienstleister zu übernehmen. Die besondere Rolle des Humankapitals als einer Einflussgröße auf die Entscheidung der etablierten Pharma-Unternehmen, die Biotechnologieunternehmen nicht zu übernehmen, betont auch Pisano (1991). Er behauptet:

„For established firms, buying biotechnology R\&D from the outside and focusing on commercialization had advantages. [...] One of the major advantages NBFs (new biotechnology firms - Anmerkung der Autorin) had over established firms was their ability to attract top scientists from the relevant academic 
fields. Established corporations were generally hard pressed to offer the combination of equity-based financial incentives and research environment sought by the academic scientists. “370

Folglich kann als Hypothese 9 festgehalten werden, dass Vertragsforschungsanbieter unter den humankapitalintensiven Unternehmen erwartet werden.

Schließlich kann darauf verwiesen werden, dass im Rahmen der Theorie der unvollständigen Verträge das Phänomen der Vertragsforschung von Aghion und Tirole (1994) explizit modelliert wurde. Die Autoren widmeten sich in ihrem Beitrag der Untersuchung der Problematik der anreizoptimalen Gestaltung einer Vertragsforschungsbeziehung. Sie gingen dabei insbesondere der Frage nach, wer die Eigentumsrechte an Erfindungen halten sollte: der FuE-Dienstleister oder der Käufer, welcher die Ergebnisse der FuE-Leistung vermarktet. Sie konnten zeigen, dass es aus der Sicht des Käufers optimal sein kann, die Eigentumsrechte an der zu erarbeitenden Erfindung dem FuEDienstleister zu überlassen und in diesem Falle eine Kofinanzierung durch einen Investor zu verlangen. ${ }^{371}$

\subsection{Strategieorientierte Modelle der Indu- strieökonomik}

Der Transaktionskosten- und der Property-Rights-Ansatz bieten einen Analyserahmen, der es ermöglicht zu untersuchen, unter welchen Bedingungen bestimmte Transaktionen vertikal in einer Firma integriert bzw. zwischen selbstständigen Akteuren abgewickelt werden. Die Grundüberlegungen dieser Ansätze gelten jedoch klassischerweise der Analyse von Integrations- und Desintegrationsentscheidungen im Bereich der klassischen Güter. Die Ressourcenallokation im FuE-Bereich zeichnet sich jedoch durch einige Besonderheiten aus, die bei der Analyse des Phänomens der Vertragsforschung nicht unbeachtet bleiben können. Insbesondere ist die FuE-Tätigkeit durch das Vorliegen von Externalitäten gekennzeichnet: Es kann davon ausgegangen werden, dass die Spillover-Problematik nicht nur die Entscheidungen der Akteure im Hinblick

370 Pisano (1991), S. 240.

371 Zur Anwendung des Theorierahmens des Property-Rights-Ansatzes auf den Kontext der FuE-Kooperationen vgl. auch Rosenkranz/Schmitz (1999), Adams/Chiang/Jensen (2003). 
auf die Aufnahme und Ausweitung der unternehmensinternen FuE-Tätigkeit beeinflusst, sondern auch Auswirkungen auf Organisationsstrukturen der industriellen FuE-Tätigkeit hat.

Strategieorientierte Ansätze der Industrieökonomik widmen sich explizit der Modellierung von Organisationsentscheidungen der Wirtschaftsakteure im FuE-Bereich und berücksichtigen dabei die Problematik der unvollständigen Appropriierung des erarbeiteten Wissens. Der Rückgriff auf die industrieökonomischen Modelle der Kollusion im FuE-Bereich ermöglicht daher eine vertiefte Analyse der Bedeutung von positiven Externalitäten der FuE-Tätigkeit.

Zwei zentrale Konzepte der industrieökonomischen Literatur sollen nachfolgend beleuchtet werden. In Abschnitt 4.4.1 wird zunächst auf die Modellierung des Phänomens der Externalitäten im FuE-Bereich eingegangen. Anschließend wird in Abschnitt 4.4.2 der von Cohen und Levinthal eingeführte Begriff der "Absorptionsfähigkeit" näher erläutert. Im Mittelpunkt der Analyse in Abschnitt 4.4.3 steht die Formulierung der Implikationen industrieökonomischer Erkenntnisse für die Analyse des Phänomens der Vertragsforschung.

\subsubsection{Spillovers und FuE-Kooperation}

Strategieorientierte Ansätze der Industrieökonomik modellieren meist horizontale Kooperationen, d.h. Kooperationen zwischen Unternehmen, die auf dem Absatzmarkt im direkten Wettbewerb stehen. Die meisten Modelle bauen auf den Arbeiten von d'Aspremont und Jacquemin $(1988,1990)$ auf, indem sie Bedingungen für die relative Überlegenheit bestimmter Kooperations- bzw. Wettbewerbsstrategien vor dem Hintergrund technologischer Externalitäten im Rahmen zwei- oder dreistufiger Cournot- bzw. Bertrand-Modelle untersuchen.

Grundlegend für alle auf den Arbeiten von d'Aspremont und Jacquemin aufbauenden Modelle zur Beschreibung von FuE-Kooperationen ist die Annahme positiver Externalitäten der FuE-Tätigkeit. Vorausgesetzt wird, dass einzelne Unternehmen die Ergebnisse ihrer eigenen Forschungsanstrengungen nicht vollständig aneignen können, da ein Teil des FuE-relevanten Wissens stets Wettbewerbern zufließt. Die Konkurrenten sind in der Lage, dieses aus ihrer Sicht extern zugängliche Wissen intern umzusetzen und $\mathrm{zu}$ verwerten.

Kostenreduzierende Modelle der FuE-Kooperation beschreiben das Verhalten von Unternehmen, die in ihrer Forschungspolitik das Ziel der Prozessinnovationen verfolgen, d.h. ihre Forschung auf das Hervorbringen von kostensenkenden Inventionen ausrichten. Positive Wissensexternalitäten werden dabei 
derart beschrieben, dass alle Unternehmen des relevanten Marktes von der Forschungstätigkeit der Wettbewerber profitieren, indem sie einen Teil ihrer Forschungsergebnisse aneignen können. Der aneigenbare Anteil des extern zugänglichen Wissens wird durch den Parameter $\beta$ vorgegeben.

Neben der Zulassung der unvollständigen Appropriierbarkeit können folgende Gemeinsamkeiten der in diesem Unterkapitel zu erläuternden Modelle identifiziert werden: ${ }^{372}$

- Es wird zur Vereinfachung eine deterministische FuE-Erfolgsfunktion unterstellt, d.h. es wird vorausgesetzt, dass die Ergebnisse von FuE nicht zufallsabhängig sind, sondern dass die geleisteten FuE-Investitionen immer eine wirtschaftlich verwertbare Invention zur Folge haben. Kurz gefasst: Interne Unsicherheit wird ausgeschlossen. ${ }^{373}$

- Kollusion ist industrieweit, d.h. schließt alle Unternehmen ein, die sonst im Wettbewerb im FuE-Bereich stünden. Die Unternehmen sind symmetrisch.

- Die Analyse ist wohlfahrtsorientiert in dem Sinne, dass vorwiegend die effektiven Forschungsanstrengungen und Outputentscheidungen der Unternehmen unter alternativen Szenarien analysiert werden.

Um Verständnis für die Grundannahmen und Struktur kostenreduzierender Modelle der FuE-Kooperation zu entwickeln, soll zunächst auf das Grundmodell von d'Aspremon und Jacquemin (1988) genauer eingegangen werden (Abschnitt 4.4.1.1). Im Anschluss daran wird auf die Besonderheiten der Modellierung von technologischen Externalitäten in späteren Modellen eingegangen, ohne dass die diesen Modellen zugrunde liegende mathematische Formulierung dargelegt wird (Abschnitt 4.4.1.2). Der Schwerpunkt wird statt dessen auf die Erläuterung der Modellergebnisse gelegt.

\subsubsection{Das Grundmodell von d'Aspremont und Jacquemin}

Beim Modell von d'Aspremont und Jacquemin (AJ-Modell) handelt es sich um eine adaptierte, zweistufige Form des homogenen Cournot-Modells. ${ }^{374}$ Zwei

372 Vgl. Veugelers (1998), S. 423 f.

373 Zur Definition der internen Unsicherheit vgl. Abschnitt 2.1.

${ }^{374}$ Für das Grundmodell vgl. d'Aspremont/Jacquemin (1988). Für die Untersuchung der Stabilitätsbedingungen vgl. Henriquez (1990) und d'Aspremont/Jacquemin (1990). Eine Be- 
Unternehmen $(i, j=1,2, i \neq j)$ legen in der ersten Spielstufe simultan die Höhe ihrer FuE-Budgets und damit die erreichbaren Kostenreduktionen $\left(x_{i}\right)$ fest. Gegeben die Entscheidungen im FuE-Bereich, bestimmen sie dann in der zweiten Spielstufe die Cournot-Produktionsmengen $\left(q_{i}\right) \cdot{ }^{375}$ Beide Unternehmen berücksichtigen dabei, dass ein Teil ihrer Forschungsergebnisse dem Wettbewerber zufließt und zur Reduktion seiner Produktionskosten führt. Das Ausmaß des kostensenkenden Effekts der Forschungstätigkeit des Konkurrenten wird durch den Wert des Spillover-Parameters $(\beta, 0 \leq \beta \leq 1)$ bestimmt. Die Produktionskosten des Unternehmens $i\left(c_{i}\right)$ in der zweiten Spielstufe sind folglich gegeben durch

$$
c_{i}\left(q_{i}, x_{i}, x_{j}\right)=\left(A-x_{i}-\beta x_{j}\right) q_{i}
$$

wobei $x_{i}$ die dank der eigenen Forschungstätigkeit erzielte Kostenreduktion und $\beta x_{j}$ den vom Unternehmen $i$ aneigenbaren Anteil der Kostenreduktion des Wettbewerbers angibt (ausgehend vom Kostenniveau $A$ ). In der Folge gibt die Funktion

$$
X_{i}=x_{i}+\beta x_{j}
$$

die gesamte vom Unternehmen $i$ erreichte Kostenreduktion an. ${ }^{376}$ Die Kosten, die eines der beiden Unternehmen aufbringen muß, um eine Reduktion der Produktionskosten um $x_{i}$ zu erreichen, betragen $\frac{\gamma}{2} x_{i}^{2}$, wobei $\gamma$, der FuEKostenparameter, größer 0 ist.

Der Markt, auf dem die Unternehmen agieren, wird durch die inverse Nachfragefunktion $P(Q)=a-b Q, Q=q_{i}+q_{j}$ beschrieben.

D'Aspremont und Jacquemin unterziehen vier verschiedene Verhaltensszenarien einer komparativen Analyse:

Szenario 1: Wettbewerb sowohl in FuE als auch auf dem Absatzmarkt,

Szenario 2: FuE-Kooperation bei Beibehaltung der Konkurrenz auf dem Absatzmarkt,

Szenario 3: Kollusion sowohl im Forschungs- als auch im Produktionsbereich,

schreibung einer vereinfachten Version des Modells von d'Aspremont und Jacquemin bieten Bühler/Jaeger (2002), S. $130 \mathrm{ff}$.

375 Das Spiel wird mit Hilfe der Rückwärtsinduktion gelöst.

376 Vgl. Amir (2000), S. 1014. 
Szenario 4: wohlfahrtsoptimale Lösung.

Nachfolgend werden die ersten zwei Szenarien des Spieles näher betrachtet, d.h. Wettbewerb in FuE sowie auf dem Absatzmarkt und FuE-Kooperation bei Beibehaltung der Konkurrenz auf dem Absatzmarkt. ${ }^{377}$

Im 1. Fall (Szenario 1) koordinieren die Unternehmen weder die FuE- noch die Produktionsentscheidungen, daher werden beide Größen durch das Kalkül der individuellen Gewinnmaximierung bestimmt. ${ }^{378}$ Die Gewinnfunktion der Unternehmen in der zweiten Spielstufe ist gegeben durch ${ }^{379}$

$$
\Pi_{i}=\left(P(Q)-c_{i}\right) q_{i}-\frac{\gamma}{2} x_{i}^{2}
$$

Löst man die Bedingung erster Ordnung $\frac{d \Pi_{i}}{d q_{i}}=0$ nach $q_{i}$ auf ${ }^{380}$ und beachtet die symmetrische Marktstruktur, so ergibt sich als Nash-Cournot-Produktmarktgleichgewicht

$$
q_{i}=\frac{(a-A)+(2-\beta) x_{i}+(2 \beta-1) x_{j}}{3 b}
$$

Um die gewinnmaximierende FuE-Strategie herzuleiten, setzt man nun $q_{i}$ und $q_{j}\left(q_{j}=q_{i}\right)$ aus (4.24) in die Gewinnfunktion aus (4.23) ein. Die Gewinnfunktion der ersten Spielstufe ist gegeben durch

$$
\Pi_{i}^{*}=\frac{1}{9 b}\left[(a-A)+(2-\beta) x_{i}+(2 \beta-1) x_{j}\right]^{2}-\frac{\gamma}{2} x_{i}^{2}
$$

Löst man die Bedingung erster Ordnung $\frac{d \Pi_{i}^{*}}{d x_{i}}=0$ nach $x_{i}$, so erhält man die symmetrische Lösung

$$
x_{i}^{*}=\frac{(a-A)(2-\beta)}{4.5 b \gamma-(2-\beta)(\beta+1)}
$$

Die Produktionsmenge ergibt sich direkt durch Einsetzen von $x^{*}\left(x_{i}=x_{j}\right)$ in (4.24). Man erhält für $Q^{*}=q_{i}^{*}+q_{j}^{*}$

$$
Q^{*}=\frac{2(a-A)}{3 b} \frac{4.5 b \gamma}{4.5 b \gamma-(2-\beta)(\beta+1)}
$$

377 Für die Analyse aller vier Szenarien vgl. u.a. Phlips (1995), Kap. 10, S. 173-178.

378 Vgl. Bester (2004), S. 190.

${ }^{379}$ Für die nachfolgende Erläuterung vgl. Bester (2004), S. $145 f$.

${ }^{380}$ Für die Bedingungen zweiter Ordnung vgl. jeweils d'Aspremont/Jacquemin (1988). 
Im 2. Fall (Szenario 2) legen die Unternehmen ihre FuE kooperativ fest, d.h. sie maximieren in der ersten Spielstufe den gemeinsamen Gewinn

$$
\hat{\Pi}=\Pi_{1}^{*}+\Pi_{2}^{*}=\frac{1}{9 b} \Sigma_{i=1}^{2}\left\{\left[(a-A)+(2-\beta) x_{i}+(2 \beta-1) x_{j}\right]^{2}-\frac{\gamma}{2} x_{i}^{2}\right\}
$$

Löst man nun die Bedingung erster Ordnung unter Beachtung der Symmetrie auf, so erhält man direkt

$$
\hat{x}=\frac{(a-A)(\beta+1)}{4.5 b \gamma-(\beta+1)^{2}}
$$

und schließlich die Produktionsmenge

$$
\hat{Q}=\frac{2(a-A)}{3 b} \frac{4.5 b \gamma}{4.5 b \gamma-(\beta+1)^{2}}
$$

Vergleicht man $x^{*}$ aus (4.26) und $\hat{x}$ aus (4.29) und die Produktionsmengen $Q^{*}$ aus (4.27) und $\hat{Q}$ aus (4.30), so zeigt sich, dass im Falle bedeutender Spillovers $(\beta>0.5)$ sowohl die Forschungsleistung als auch der Output größer ausfällt, wenn die Unternehmen kooperieren. Dieses Ergebnis kann auf die Internalisierung externer Effekte durch die gemeinsame Festlegung der Forschungsleistung zurückgeführt werden. Partielle Kollusion (im FuE-Bereich) erweist sich damit beim Vorliegen bedeutender Spillovers aus wohlfahrtstheoretischer Sicht als überlegen gegenüber dem FuE-Wettbewerb. Es kann jedoch gezeigt werden, dass im Falle bedeutender Spillovers ein Übergreifen der Kollusion auf die Produktmärkte zur Senkung der Outputmenge (und damit zur Preissteigerung) führt. ${ }^{381}$

\subsubsection{Modellierung von Spillovers}

In der Analyse von d'Aspremont und Jacquemin wurde davon ausgegangen, dass die Spillover-Effekte bei FuE-Kollusion genauso stark ausfallen wie bei FuE-Wettbewerb. Es kann jedoch vermutet werden, dass kooperierende Unternehmen mehr Informationen austauschen, d.h. stärker von den positiven Externalitäten profitieren, als Unternehmen, die miteinander konkurrieren. ${ }^{382}$ Die Unterscheidung zwischen der gemeinsamen Festlegung von gewinnoptimalen Forschungsanstrengungen und der Entscheidung, die Ergebnisse der Forschungsarbeiten zu teilen, ist von Kamien et. al (1992) berücksichtigt worden.

381 Vgl. d'Aspremont/Jacquemin (1988), S. 1135.

382 Vgl. Kamien/Muller/Zang (1992), S. 1296, d'Aspremont/Jacquemin (1990), S. 641, Bester (2004), S. 193, Phlips (1995), S. 179. 
In ihrem auf der Arbeit von d'Aspremont und Jacquemin aufbauenden, jedoch um Produktheterogenitätsparameter $(\gamma)$ erweiterten und auf $n$ Unternehmen verallgemeinerten Modell ${ }^{383}$ unterschieden die Autoren vier Szenarien: ${ }^{384}$

- Beim FuE-Wettbewerb entscheidet jedes Unternehmen in der ersten Spielstufe über die eigenen FuE-Investitionen (gegeben das FuE-Niveau des Wettbewerbers). In der zweiten Spielstufe werden die Grenzkosten der Produktion dank der eigenen FuE-Anstrengung sowie durch den aneigenbaren Teil der FuE-Anstrengungen des Konkurrenten reduziert.

- Beim FuE-Kartell koordinieren die Unternehmen in der ersten Spielstufe ihre FuE-Entscheidungen, um die Summe der gemeinsamen Gewinne zu maximieren. In der zweiten Spielstufe profitieren sie von den technologischen Externalitäten, die Spilloverrate steigt jedoch im Vergleich zum FuE-Wettbewerb nicht an.

- Beim RJV-Wettbewerb entscheiden beide Unternehmen in der ersten Spielstufe unabhängig voneinander über ihre FuE-Investitionen, die Firmen tauschen jedoch die FuE-relevanten Informationen aus und vermeiden damit duplizierende Forschungsanstrengungen. In der zweiten Spielstufe werden die Grenzkosten der Produktion durch die Summe aller FuE-Aktivitäten reduziert (der Spilloverparameter erreicht sein maximales Niveau, $\beta=1$ ).

- Beim RJV-Kartell koordinieren die Unternehmen ihre FuEAktivitäten, um die Summe der gemeinsamen Gewinne zu maximieren und tauschen FuE-relevanten Informationen aus. Die Grenzkosten der Produktion in der zweiten Spielstufe werden durch die Summe aller FuE-Aktivitäten reduziert $(\beta=1)$.

In beiden Szenarien der Kartellisierung werden die Forschungsaufwendungen so festgelegt, dass die Summe der gemeinsamen Gewinne maximiert wird. Nur im Rahmen der research joint ventures (RJV) findet jedoch perfekter Informationsaustausch statt, wodurch duplizierende Forschung vermieden wird und es zur vollständigen Internalisierung externer Effekte der Forschungstätigkeit

383 Für eine umfassende und eingehende komparative Analyse der Modelle von d'Aspremont und Jacquemin (1988) und Kamien/Muller/Zang (1992) vgl. insbesondere Amir (2000).

${ }^{384}$ Vgl. Kamien/Muller/Zang (1992), S. 1295, Lenz (1998), S. 44. 
kommt. Im Unterschied zum Modell von d'Aspremont und Jacquemin berücksichtigen folglich Kamien et al. (1992) explizit Spielszenarien, welche sich durch die Entscheidung der Spieler für die vollständige Offenlegung der FuE-relevanten Informationen auszeichnen.

Die Modellanalyse zeigt dann, dass die erzielte Kostenreduktion im Falle bedeutender Spillovers $\left(\beta>\frac{\gamma}{2}\right)$ größer im Szenario des FuE-Kartells ausfällt als beim FuE-Wettbewerb. Dieses Ergebnis führen die Autoren auf das Vorliegen von zwei unterschiedlichen Typen von Externalitäten zurück. Die erste Art der Externalität (competitive-advantage externality) entsteht dadurch, dass die FuE-Tätigkeit eines Unternehmens nicht nur zur Senkung seiner eigenen Produktionskosten sondern auch (über die Spillover-Effekte) zur Kostenreduktion bei seinen Konkurrenten führt und in der Folge den Wettbewerb zwischen den Unternehmen verschärft. Dies wird die Anreize zur Ausweitung der eigenen FuE-Tätigkeit senken. Die zweite Art der Externalität (combined-profits externality) entsteht als Auswirkung der eigenen FuE-Tätigkeit eines Unternehmens auf die Summe der Gewinne der Wettbewerber ${ }^{385}$; sie wird beim FuEWettbewerb ignoriert und bei Kartellisierung im FuE-Bereich internalisiert. Bei entsprechend hoher Spilloverrate wird der negative Effekt der ersten Externalität vom positiven Effekt der zweiten Externalität überkompensiert. ${ }^{386}$

Die Modellanalyse zeigt ferner, dass das RJV-Kartell aus wohlfahrtstheoretischer Sicht allen analysierten Szenarien vorzuziehen ist: Es dominiert alle anderen Szenarien sowohl in Hinsicht auf die erzielten Kostenreduktionen, als auch im Hinblick auf die durch die jeweiligen Unternehmen erzielten Gewinne. ${ }^{387}$ Die Überlegenheit des RJV-Kartells im Vergleich zum RJV-Wettbewerb kann unter Rückgriff auf die Problematik des Trittbrettfahrerverhaltens (freerider effect) begründet werden. Im RJV-Wettbewerb-Szenario berücksichtigt das jeweilige Unternehmen $(i)$ bei der Entscheidung über die Höhe eigener FuEInvestitionen den antizipierten Spillover-Effekt: Steigt der Wert des SpilloverParameters an und ändern andere Unternehmen $(j \neq i)$ ihre FuE-Investitionen nicht, dann ist es aus der Sicht des jeweiligen Unternehmens vorteilhaft, die eigenen Forschungsaufwendungen zu reduzieren, um vordergründig von den Externalitäten zu profitieren. ${ }^{388}$

385 Die FuE-Investitionen des Unternehmens $i$ erhöhen seine Produktionseffizienz und haben eine Erhöhung seines Marktanteils auf Kosten der Wettbewerber zur Folge, vgl. hierzu auch Hinloopen (1997), S. 162 f.

386 Vgl. Kamien/Muller/Zang (1992), S. 1294 f. sowie S. $1299 \mathrm{ff}$.

387 Vgl. Kamien/Muller/Zang (1992), S. 1302.

388 Vgl. Kamien/Muller/Zang (1992), S. 1302. 
Im Hinblick auf die Herleitung der wirtschaftspolitischen Implikationen bedeutet dies, dass die Bildung von joint ventures im FuE-Bereich befürwortet werden kann, wenn zwei Bedingungen erfüllt werden: a) die Partner sollen ihre gemeinsamen Gewinne aus der FuE-Tätigkeit maximieren (wie im Szenario des RJV-Kartells) und zugleich b) im Produktionsbereich nicht kollidieren. ${ }^{389}$

Poyago-Theotoky (1999) ging in ihrem Beitrag von der Beobachtung aus, dass in den meisten Modellen der FuE-Kooperation die technologischen Externalitäten (vorgegeben durch den Parameter $\beta$ ) exogenen bestimmt sind. Sie behauptete jedoch, dass die Möglichkeiten der einzelwirtschaftlichen Einflussnahme auf die Höhe des Parameters $\beta$ zugelassen werden sollte, denn "firms have some power in deciding how much of the information/new knowledge they create becomes publicly available and hence useful to their competitors. “390 Daher erweiterte sie das AJ-Modell um eine zusätzliche Spielstufe, in der die Unternehmen, nachdem sie die Höhe der FuE-Investitionen festgelegt haben, Entscheidungen darüber treffen, inwieweit sie die Ergebnisse ihrer FuE-Tätigkeit teilen. Analysiert wurden dann zwei Spielszenarien: im ersten Fall stehen die Unternehmen in allen Spielstufen im Wettbewerb; im zweiten Fall koordinieren sie ihre Entscheidungen bezüglich der Höhe der zu tätigenden FuE-Investitionen und - in der zweiten Spielstufe - bezüglich des Anteils der auszutauschenden FuE-Resultate (disclosure rate).

Die Modellanalyse zeigte, dass Kooperation im FuE-Bereich nicht nur zu höheren erreichten FuE-Kostenreduktionen führt, sondern auch, dass die die gemeinsamen Gewinne maximierenden Unternehmen dazu veranlasst werden, alle relevanten FuE-Ergebnisse auszutauschen. Da in diesem Falle die Gewinne pro Firma höher ausfallen, werden die Unternehmen immer einen Anreiz haben, eine FuE-Kooperation einzugehen. Wird dagegen keine FuE-Kooperation angestrebt, dann werden ihre Gewinne maximiert, wenn sie sich gegen eine Offenlegung von FuE-Ergebnissen entscheiden. Wird folglich die Möglichkeit der strategischen Einflussnahme auf den Wert des Spillover-Parameters zugelassen, so zeigt sich, dass kooperierende Unternehmen, die ihre Gewinne maximieren wollen, alle relevanten FuE-Resultate teilen sollten.

Hinloopen (2003) vertiefte die Analyse der Bedeutung von Spillovers für die Entscheidung der Akteure im Bereich der Organisation von FuE. Er ging dabei von der Einschätzung aus, dass die Modelle von d'Aspremont und Jacquemin

389 Vgl. Phlips (1995), S. 179.

390 Poyago-Theotoky (1999), S. 254. 
(AJ-Modell) und von Kamien, Muller und Zang (KMZ-Modell) die drei folgenden Schwächen in Hinsicht auf die Modellierung von Spillovers aufweisen. ${ }^{391}$

Erstens stellte er fest, dass im AJ-Modell Spillovers auftreten, nachdem der FuE-Prozess beendet wurde, d.h. es diffundieren FuE-Resultate und nicht der FuE-Input. ${ }^{392} \mathrm{Er}$ argumentierte, dass nicht nur von der Diffusion der FuEErgebnisse sondern auch vom Vorliegen von Spillovers während der FuE-Phase ausgegangen werden müsste.

Zweitens verwies er auf die implizite Annahme der perfekten Additivität der FuE-Anstrengungen sowohl im AJ-Modell als auch im KMZ-Modell. Diese Annahme hielt er für nicht haltbar aus folgenden Gründen. Eine vollständige Beseitigung von Duplikation im Rahmen der FuE-Kooperation würde sich weitestgehend als unmöglich erweisen, weil (1) die Forschungsanstrengungen einzelner Unternehmen nicht zwangsläufig die gleichen Pfade verfolgen, sodass nicht alle Forschungsergebnisse einer Unternehmung von unmittelbarer Relevanz für andere Unternehmen seien, (2) Unterschiede in Forschungsstrategien, Unternehmenskultur und interner Organisation der FuE-Tätigkeit für die Unmöglichkeit der reibungslosen und vollständigen Aneignung der Forschungsergebnisse anderer Unternehmen sorgen würden.

Drittens verwies er auch auf die Unhaltbarkeit der Annahme der perfekten Additivität der FuE-Resultate (im AJ-Modell) in Kombination mit den ebenfalls vorausgesetzten fallenden Grenzerträgen der FuE-Tätigkeit. Bei hohen Spilloverraten hätte dies zur Folge, dass bei unterschiedlichen FuE-Investitionen der Unternehmen es sich aus der Sicht der Firma mit höherem FuE-Niveau lohnen würde, die zusätzlichen Geldmittel dem Wettbewerber zur Verfügung zu stellen. Da das andere Unternehmen in diesem Fall von einem relativ niedrigerem Niveau investierte, würde der zusätzliche Input für höhere FuE-Erträge sorgen. Diese könnten dann - bei Annahme perfekter Additivität - vollständig von der finanzierenden Einheit internalisiert werden. ${ }^{393}$

In seinem Modell wählte er folglich die im KMZ-Modell verwendete Spezifikation der FuE-Produktionsfunktion, die im Unterschied zu der Spezifikation im

\footnotetext{
391 Vgl. Hinloopen (2003), S. 109 f.

392 Aneigenbar ist im AJ-Modell die von dem jeweils anderen Unternehmen erzielte $\mathrm{Ko}$ stenreduktion, d.h. das Ergebnis der FuE-Anstrengungen, vgl. hierzu auch Amir (2000), S. 1014.
}

${ }^{393}$ Vgl. Hinloopen (2003), S. 110, Amir (2000), S. 1028. 
AJ-Modell das Vorliegen von Spillovers in der FuE-Phase sicherstellt. ${ }^{394}$ Ferner führte er in seinem Modell zusätzlich eine obere Schranke für den Wert des Spillover-Parameters ein $(\hat{\beta}<1)$, und schloss damit die perfekte Additivität aus. Zusätzlich unterschied er zwischen dem Spillover-Parameter $\beta$ $(\beta \in[0, \hat{\beta}))$ im Szenario des FuE-Wettbewerbs und dem Spilloverparameter $\theta(\theta \in[0, \hat{\beta}))$ im Szenario des FuE-Kartells. Um zu gewährleisten, dass die Spillover-Effekte zwischen kooperierenden Unternehmen nicht kleiner als die Spillover-Effekte bei konkurrierenden Firmen ausfallen, ließ er zudem $\theta \geq \beta$ gelten. Im Gegensatz zu Kamien et al. ermöglichte er damit einen Anstieg der technologischen Spillovers beim FuE-Kartell im Vergleich zum FuE-Wettbewerb.

Die Modellanalyse bestätigte das zuvor von d'Aspremont und Jacquemin sowie Kamien et al. erhaltene Ergebnis, dass bei erheblichen Spilloverraten die Forschungsanstrengungen und folglich die erzielten Kostenreduktionen im Falle des kooperativen Verhaltens im FuE-Bereich höher ausfallen als im Szenario des FuE-Wettbewerbs. Allerdings zeigte sich zugleich, dass bei kleineren Werten des Spillover-Parameters die FuE-Kooperation auch zu höheren FuEAufwendungen führen kann, vorausgesetzt, dass es zu einer hinreichend starken Erhöhung des Spillover-Parameters dank der Kooperation kommt. Dies Ergebnis kann erklärt werden mit dem Hinweis auf die zweite Art der von Kamien et al. erläuterten Externalität (combined-profits externality). Die kooperierenden Unternehmen sind sich der Tatsache bewusst, dass eine steigende SpilloverRate den gesamten Gewinn erhöht. ${ }^{395}$

Hinloopen kann daher folgende wirtschaftspolitischen Implikationen formulieren:

\begin{abstract}
"Rather than acquiring only as good an approximation of technological spillovers as possible in order to assess the desirability of the formation of a particular R\&D cooperative, policy makers, in addition, have to assess the likely increase in technological spillover between potential $R \& D$ partners due to the cooperative aggreement. Indeed, in case technological spillovers are small but this incresae it thought to be large „enough“, there is still good reason to allow the formation of the proposed cooperative." 396
\end{abstract}

\footnotetext{
${ }^{394}$ In dem KMZ-Modell gibt die Funktion $X_{i}$ aus (4.22) nicht die insgesamt erzielte Kostenreduktion, sondern die gesamten FuE-Investitionen (effective R\&D investment) des Unternehmens $i$ an.

395 Vgl. Hinloopen (2003), S. 111.

396 Vgl. Hinloopen (2003), S. 112.
} 


\subsubsection{Zur Frage der Absorptionsfähigkeit}

In diesem Abschnitt soll der Begriff der "Absorptionsfähigkeit“ (absorptive capacity) aufgegriffen werden. Die Einführung dieses Konzepts in die industrieökonomische Debatte geht auf Cohen und Levinthal zurück. In Abschnitt 4.4.2.1 sollen daher zunächst die Überlegungen dieser Autoren skizziert werden. Anschließend wird in Abschnitt 4.4.2.2 gezeigt, wie das Phänomen der Absorptionsfähigkeit in die industrieökonomischen Modelle der FuE-Kooperation von Kamien und Zang (2000) integriert wurde.

\subsubsection{Die Grunderkenntnisse von Cohen und Levinthal}

In ihrem im Jahre 1990 veröffentlichten Beitrag „Absorptive capacity: a new perspective on learning and innovation" führten Cohen und Levinthal in die innovationsökonomische Literatur das Konzept der Absorptionsfähigkeit ein. Als absorptive capacity definierten sie die Fähigkeit der Wirtschaftsakteure, den Wert des im Umfeld einer Organisation zugänglichen Wissens zu erkennen und die unternehmensextern vorliegenden innovationsrelevanten Wissensressourcen für betriebsinterne Zwecke zu übernehmen und verwerten. ${ }^{397}$ Damit distanzierten sie sich von der Annahme, dass das im technologischen Umfeld eines Unternehmens vorhandene Wissen kostenfrei übernommen und angeeignet werden kann; sie widmeten sich eingehend der Identifizierung von Bedingungen, die erfüllt sein müssen, damit die Übernahme und Verwertung externen Wissens erfolgreich stattfinden kann.

Die Fähigkeit der Wirtschaftsakteure, externe Informationsquellen adäquat einzuschätzen und zu verwerten, sahen die Autoren insbesondere als Funktion des internen Wissensbestandes einer Organisation. Sie behaupteten, dass die unternehmensinterne FuE-Tätigkeit der Wirtschaftsakteure zwei Funktionen erfülle. Sie dient an erster Stelle der betriebsinternen Erarbeitung technologischer Neuerungen. Das dank der internen Auseinandersetzung mit technologischen Fragestellungen angesammelte Wissen bildet jedoch zugleich die Grundlage für die Fähigkeit zur Übernahme der extern vorhandenen wissenschaftlichen Erkenntnisse. ${ }^{398}$ Erst das kontext-spezifische Know-how ${ }^{399}$ befähigt die Akteure, verwandte, unternehmensextern erarbeitete Erkenntnisse valide in

397 Vgl. Cohen/Levinthal (1990), S. 128.

$398 \mathrm{Vgl}$. Cohen/Levinthal (1990), S. 138.

399 Die Autoren weisen explizit darauf hin, dass ein Teil des Wissensbestandes einer Organisation impliziter Natur sein kann, vgl. Cohen/Levinthal (1990), S.135. 
Hinblick auf ihre Eignung für eigene Zwecke zu bewerten und im Bedarfsfall aktiv anzuwenden. Die Autoren verwiesen zugleich auf die Bedeutung der in der Produktion angesammelten Erfahrung: „through direct involvement in manufacturing, a firm is better able to recognize and exploit new information relevant to a particular product market." 400 Absorptionsfähigkeit sahen sie nicht nur als „Nebenprodukt" der internen FuE-Tätigkeit, sondern auch als „a byproduct of a firm's manufacturing operations ${ }^{\text {“401 }}$.

Die Autoren verwiesen in ihrem Beitrag zugleich auf die Gefahren der Pfadabhängigkeiten, die entstehen, wenn man vorhandene Absorptionsfähigkeiten als eine notwendige Bedingung für die Integration unternehmensextern erarbeiteter technologischer Wissensfortschritte auffasst. Sie argumentierten hierbei folgendermaßen: Absorptionsfähigkeit, die ihrerseits an die spezifische Erfahrungs- und Wissensstruktur einer Organisation gekoppelt ist, predeterminiert natürlicherweise die Art der aneigenbaren externen Wissensressourcen. Sind interne Absorptionsfähigkeiten auf bestimmten Technologiefeldern organisationsintern nicht vorhanden, können relevante technologische Fortschritte nicht in das interne FuE-Programm integriert werden. Auf Gebieten, die durch schnelle Entwicklungs- und Wachstumsraten gekennzeichnet sind, kann es infolgedessen zu einem Effekt kommen, der von den Autoren als lockout bezeichnet wird: Das Fehlen von frühen Investitionen in Absorptionsfähigkeit macht die Integration und Verwertung extern zugänglichen Wissens unmöglich. Von den technologischen Möglichkeiten kann nun nicht profitiert werden; damit steigt zunehmend das zum späteren Zeitpunkt notwendige Investitionsvolumen und die Attraktivität eines bestimmten Technologiefeldes reduziert sich. Das Unternehmen verbleibt auf dem bisherigen technologischen Pfad, auch wenn nachträglich das Potenzial der neuen Entwicklung erkannt wird. Dieses Unternehmen verliert damit den Anschluss an die neue Technologie oder - in Worten von Cohen und Levinthal - ist ausgeschlossen (locked-out) von den nachfolgenden technologischen Entwicklungen. ${ }^{402}$ Im Ergebnis stellen die Autoren fest: "lack of investment in an area of expertise early on may foreclose the future development of a technical capability in that area." 403

400 Cohen/Levinthal (1990), S. 129.

401 Cohen/Levinthal (1990), S. 129.

$402 \mathrm{Vgl}$. Cohen/Levinthal (1990), S. $136 \mathrm{ff}$.

403 Cohen/Levinthal (1990), S. 128. 


\subsubsection{Die Modellierung der Absorptionsfähigkeit}

Das Phänomen der Absorptionsfähigkeit wurde von Kamien und Zang (2000) in die Modellierung von Kooperationsentscheidungen der Wirtschaftsakteure integriert. Ausgehend von der „intuitiven Plausibilität und empirischen Stichhaltigkeit “ ${ }^{404}$ der Annahme, dass für die Übernahme unternehmensextern vorliegender Wissensressourcen die interne Absorptionsfähigkeit eine notwendige Bedingung darstellt, widmeten sie sich explizit der Erfassung des Phänomens der Absorptionsfähigkeit im Rahmen der Funktion der effektiven Forschungsleistung (effective RED effort) eines Unternehmens. Sie gingen dabei von der Hypothese von Cohen und Levinthal aus, dass die Absorptionsfähigkeit eines Unternehmens als eine Funktion seiner Forschungstätigkeit aufgefasst werden kann und schlugen folgende Spezifizierung vor:

$$
A C_{i}=\left(1-\delta_{i}\right) x_{i}^{\delta_{i}}
$$

Die Absorptionsfähigkeit $A C$ des Unternehmens $i$ fassten sie folglich als eine Funktion seiner Forschungsleistung $\left(x_{i}\right)^{405}$ und der gewählten FuE-Strategie $\left(\delta_{i}\right)$ auf. ${ }^{406}$ Eine FuE-Strategie, die auf die Verfolgung allgemeiner Erkenntnisfortschritte bzw. auf die Auseinandersetzung mit generellen technologischen Problemstellungen abstellt, entspricht $\delta=0$ und maximiert die Absorptionsfähigkeit eines Unternehmens. Eine FuE-Strategie, die auf das Hervorbringen von firmensspezifischem Wissen abzielt, entspricht dagegen $\delta=1$. Widmet sich ein Unternehmen ausschließlich der Erarbeitung spezifischer, nur unternehmensintern relevanter Wissensressourcen, so ist es nicht in der Lage, extern zugängliches Wissen für betriebsinterne Zwecke zu internalisieren $\left(A C_{i}=0\right)$.

Die Funktion der effektiven Forschungsleistung einer Unternehmung wurde folglich gegeben durch:

$$
X_{i}=x_{i}+\left(1-\delta_{i}\right)\left(1-\delta_{j}\right) \beta x_{i}^{\delta_{i}} x_{j}^{1-\delta_{i}}
$$

d.h. erneut als die Summe interner Forschungstätigkeit $\left(x_{i}\right)$ und des aneigenbaren Teils der Forschungsleistung des Wettbewerbers (zweiter Term). Der aneigenbare Teil der Forschungsleistung des Wettbewerbers variiert jedoch dabei in Abhängigkeit von der eigenen Absorptionsfähigkeit $\left(\left(1-\delta_{i}\right) x_{i}^{\delta_{i}}\right)$, dem Wert des

${ }^{404}$ Vgl. Kamien/Zang (2000), S. 996.

405 Die Variable $x_{i}$ repräsentiert in dem KMZ-Modell die FuE-Investitionen des Unternehmens und im AJ-Modell die erzielte Kostenreduktion.

406 Vgl. Kamien/Zang (2000), S. 997 f. 
Spillover-Parameters $(\beta)$, der von dem Wettbewerber gewählten FuE-Strategie $\left(\delta_{j}\right)$ und seinem Investitionsvolumen $\left(x_{j}\right)$.

Wählen nun beide Unternehmen ( $i$ und $j, i \neq j$ ) eine breite FuE-Strategie $\left(\delta_{i}=\delta_{j}=0\right)$, so reduziert sich (4.32) zu:

$$
X_{i}=x_{i}+\beta x_{j}
$$

einer Funktion, die in den bisherigen Modellen der FuE-Koooperation verwendet wurde (vgl. Formel 4.22 im AJ-Modell).

Wählt das Unternehmen $i$ eine FuE-Strategie, die auf die Erarbeitung nur unternehmensintern relevanter Wissensressourcen abstellt, dann reduziert sich (4.32) zu $X_{i}=x_{i}$, d.h. das Unternehmen profitiert nicht von den Forschungsanstrengungen der Wettbewerber aufgrund fehlender Absorptionsfähigkeit.

Die Berücksichtigung der strategischen Variable $\delta$ machte die Erweiterung des zweistufigen Spieles von d'Aspremont und Jacquemin um eine weitere Spielstufe notwendig. In der Folge stellten Kamien und Zang ein dreistufiges Spiel auf: In der ersten Spielstufe entscheiden die Unternehmen simultan über ihre FuE-Strategie $\delta$. In der zweiten Spielstufe legen sie simultan die Höhe ihrer FuE-Investitionen (und damit der erreichbaren Kostenreduktion, $x$ ) fest. In der dritten Spielstufe werden schließlich die Cournot-Produktionsmengen simultan festgelegt $(q) \cdot{ }^{407}$ Das Spiel wird erneut mit Hilfe der Rückwärtsinduktion gelöst.

Vier Szenarien des Spieles wurden von den Autoren betrachtet: Im ersten Fall treffen beide Unternehmen ihre FuE-Entscheidungen unabhängig voneinander sowohl in der ersten als auch zweiten Spielstufe. Im zweiten Fall findet in beiden Spielstufen eine Kooperation statt. Im dritten Szenario treffen die Unternehmen ihre Entscheidungen über die FuE-Strategien unabhängig voneinander, koordinieren jedoch ihre FuE-Investitionen. Im letzten Fall koordinieren die Unternehmen ihre FuE-Strategien aber nicht ihre FuE-Investitionen. Die Bezeichnung research joint venture (RJV) verwendeten die Autoren nun zur Beschreibung einer Strategie mit der Kooperation in der zweiten Spielstufe. ${ }^{408}$

Die Modellanalyse zeigt, dass wenn die Unternehmen nicht kooperieren, d.h. ihre Forschungsleistung $\left(x_{i}\right)$ im Hinblick auf die Maximierung eigener Gewinne festlegen, dann wählen sie im Gleichgewicht eine FuE-Strategie, die auf die Erarbeitung firmenspezifischer Wissensressourcen $\left(\delta_{i}=\delta_{j}=1\right)$ abstellt.

407 Vgl. Kamien/Zang (2000), S. 998 f.

$408 \mathrm{Vgl}$. Kamien/Zang (2000), S. 1000. 
Dieses Ergebnis gilt unabhängig davon, ob sie in der ersten Spielstufe ihre FuEStrategie koordinieren oder nicht. Sie entscheiden sich bei Nicht-Kooperation für eine generelle FuE-Strategie $\left(\delta_{i}=\delta_{j}=0\right)$ nur dann, wenn keine Externalitäten der FuE-Tätigkeit bestehen $(\beta=0)$. Intuitiv können diese $\mathrm{Er}$ gebnisse folgendermaßen begründet werden. Die Spillover-Effekte können durch die Wahl einer FuE-Strategie reduziert werden, welche auf eine Erarbeitung firmenspezifischer Wissensressourcen abstellt und folglich die Externalitäten der FuE-Tätigkeit eingrenzt. Eine breite FuE-Strategie wird nur dann gewählt, wenn keine Gefahr der Externalitäten besteht. ${ }^{409}$

Kooperieren die Unternehmen in der zweiten Spielstufe, d.h. treffen sie in der zweiten Spielstufe Entscheidungen im Hinblick auf die Maximierung gemeinsamer Gewinne, dann, unabhängig davon, ob sie in der ersten Spielstufe kooperieren oder nicht, stellt die Wahl der breiten FuE-Strategie $(\delta=0)$ das einzige symmetrische teilspielperfekte Gleichgewicht dar. ${ }^{410}$ Diese Ergebnisse verdeutlichen zugleich, dass die Wahl der verfolgten FuE-Strategie von der Entscheidung für oder gegen ein research joint venture abhängt: Wird ein RJV angestrebt, dann soll im Hinblick auf die Gewinnmaximierung eine generelle FuE-Strategie gewählt werden. Die Autoren verweisen in diesem Zusammenhang auf die Ähnlichkeit ihrer Ergebnisse mit den Ergebnissen der Analyse von Poyago-Theotoky. Der Unterschied besteht lediglich darin, dass die Entscheidung, die FuE-Resultate eigener FuE-Tätigkeit dem Partner zugänglich zu machen, in dem Modell von Kamien und Zang mit der Wahl einer generellen FuE-Strategie verbunden ist. ${ }^{411}$

\subsubsection{Implikationen im Kontext der Vertragsforschung}

Die in diesem Abschnitt erläuterten kostenreduzierenden Modelle der FuEKooperation analysieren die Entscheidungen der Wirtschaftsakteure vor dem Hintergrund der technologischen Externalitäten der FuE-Tätigkeit. Die Berücksichtigung der Spillover-Problematik führt in diesen Modellen zur Unterscheidung zwischen dem kostensenkenden Effekt eigener FuE-Leistung eines Unternehmens $\left(x_{i}\right)$ und dem gesamten FuE-Effekt $\left(X_{i}\right)$, der als Summe der eigenen FuE-Leistung und des aneigenbaren Teils der Forschungsleistung

409 Vgl. Kamien/Zang (2000), S. 1006.

$410 \mathrm{Vgl}$. Kamien/Zang (2000), S. 1008.

411 Vgl. Kamien/Zang (2000), S. 1008. 
der Wettbewerber $\left(\beta x_{j}\right)$ zustande kommt. Es kann im Rahmen dieser Modelle gezeigt werden, dass kooperative FuE-Niveaus im Falle bedeutender Spillovers höher als nicht-kooperative FuE-Investitionen ausfallen. Während folglich die unvollständigen Aneignungsmöglichkeiten die Anreize zur Aufnahme und Ausweitung der eigenen FuE-Tätigkeit senken, führt die Internalisierung bedeutender externer Effekte in einer FuE-Kooperation zu einem Anstieg der Forschungsanstrengungen der Unternehmen.

Ferner zeigen sowohl Kamien et al. (1992) als auch Poyago-Theotoky (1999) explizit, dass die im FuE-Bereich kooperierenden Unternehmen, welche die Entscheidung für die vollständige Offenlegung von FuE-relevanten Informationen treffen, höhere Gewinne erreichen können. Es stellt sich folglich die Frage, warum kooperatives Verhalten mit vollständiger Informationsoffenlegung, welches im Hinblick auf die erzielbaren Gewinne dem nicht-kooperativen Verhalten vorzuziehen ist, nicht von allen Unternehmen gewählt wird. In diesem Zusammenhang verweisen Kamien und Zang explizit auf das Phänomen der Transaktionskosten. ${ }^{412}$

Obwohl folglich eine Kooperation aus wohlfahrtstheoretischer Sicht befürwortet werden kann, wenn von erheblichen Spilloverraten ausgegangen wird und aus einzelwirtschaftlicher Sicht unter gleicher Bedingung aufgrund der Gewinnsteigerung angestrebt werden sollte, können sich die Wirtschaftsakteure gegen diese Option entscheiden. Dies verdeutlicht die Problematik der Zusammenarbeit im FuE-Bereich: während die Gewinnsituation der Unternehmen bei einer Entscheidung für den Austausch von allen relevanten FuE-Informationen verbessert wird, kann die Erwartung des opportunistischen Verhaltens zu einer Entscheidung gegen die Offenlegung eigenen Wissens führen.

Der Hinweis der industrieökonomischen Modelle auf die Möglichkeit einer strategischen Einflussnahme auf die Höhe des Spillover-Parameters hat Konsequenzen für die empirische Analyse der Determinanten der Vertragsforschung. In vielen empirischen Studien werden die Externalitäten der FuE-Tätigkeit auf der Branchenebene erfasst, d.h. es wird letztlich davon ausgegangen, dass einzelne Unternehmen eines Technologiefeldes nur begrenzte Möglichkeiten haben, die Spillovereffekte ihrer eigenen FuE-Tätigkeit zu beeinflussen. Die Autoren der dargestellten Modelle weisen jedoch explizit darauf hin, dass der einzelwirtschaftliche Einfluss nicht a priori ausgeschlossen werden kann. So bemerken beispielweise d'Aspremont und Jacquemin, dass SpilloverEffekte unterschiedlich stark ausfallen können in Abhängigkeit davon, ob sich

${ }^{412}$ Vgl. Kamien/Zang (2000), S. 1009. 
die Unternehmen auf Forschungs- oder auf anwendungsnahe Entwicklungsaktivitäten konzentrieren. ${ }^{413}$ In einem Modell von Vonortas (1994) führt dies sogar zu der Annahme, dass Externalitäten nur bei Forschungsaktivitäten auftreten können, nicht jedoch bei Entwicklungsaktivitäten, denn „,[d]evelopment research creates firm-specific knowledge which cannot be transferred at a reasonable cost." ${ }^{414} \mathrm{Da}$ davon auszugehen ist, dass verschiedene Unternehmen unterschiedliche Anteile der Grundlagenforschung, angewandter Forschung und experimenteller Entwicklung am gesamten FuE-Budget haben können, sollen die Externalitäten der FuE-Tätigkeit in der vorliegenden Arbeit stets auf Unternehmensebene erfasst werden.

Von zentraler Bedeutung für die vorliegende Studie ist der Hinweis der industrieökonomischen Literatur auf die grundlegende Rolle der Absorptionsfähigkeit als einer Voraussetzung für die Übernahme relevanter Wissensressourcen aus dem technologischen Umfeld einer Unternehmung. Diese Erkenntnis, welche von Kamien und Zang (2000) im Zusammenhang mit der FuE-Kooperation erläutert wurde, gilt desto mehr im Kontext der Vertragsforschungsbeziehungen. Wird die Annahme zugelassen, dass Integration und Verwertung unternehmensextern erarbeiteten Wissens nur dann erfolgreich sein kann, wenn auch ein Mindestniveau an betriebsinternen FuE-Kapazitäten vorhanden ist, dann muss erneut (vgl. Abschnitt 4.3.4.2) das Potenzial der zu eigener FuE-Tätigkeit substitutiven Auftragsvergabe als gering eingeschätzt werden. Lässt man die Notwendigkeit interner Absorptionsfähigkeit gelten, dann ist davon auszugehen, dass der Auftragsvergabe eine gewichtige Rolle insbesondere als Instrument der komplementären Wissensbeschaffung zukommt.

Für den empirischen Teil der Arbeit sollen die obigen Ausführungen in der folgenden Form festgehalten werden:

Hypothese 10 Für die Auftragsvergabe stellt die interne Absorptionsfähigkeit eine notwendige Bedingung dar.

Cohen und Levinthal (1990) gingen in ihrem Beitrag davon aus, dass Absorptionsfähigkeit insbesondere als Funktion eigener FuE-Tätigkeit aufgefasst werden kann. Kamien und Zang (2000) verwiesen in ihrem Beitrag zudem darauf, dass bei der Erfassung der Absorptionsfähigkeit verstärkt auf das im Laufe

413 Vgl. d'Aspremont/Jacquemin (1990), S. 641.

414 Vonortas (1994), S. 417. 
der FuE-Tätigkeit angesammelte Wissen und die gemachten Erfahrungen abgestellt werden sollte: „a firm's absorptive capacity may be more explicitly recognized to result from its cumulative R\&D activity over time ${ }^{415}$. Daher wird in der vorliegenden Arbeit zur Operationalisierung des Konzepts der Absorptionsfähigkeit die Eigenschaft der Unternehmen herangezogen, FuE-Tätigkeit auf kontinuierlicher Basis zu betreiben.

\subsection{Zusammenfassung und abschließende Be- merkungen}

Nachfolgen sollen die zentralen Ergebnisse der theoriegeleiteten Auseinandersetzung mit dem Phänomen der Vertragsforschungsaktivitäten zusammengefasst werden. In Abschnitt 4.5.1 werden zunächst einige Ähnlichkeiten und Unterschiede herausgearbeitet, die sich bei der Betrachtung dieses Phänomens aus der Perspektive der drei verschiedenen Forschungsrichtungen ergeben. In Abschnitt 4.5.2 werden die im Rahmen der Analyse formulierten Hypothesen zusammengestellt und abschließend diskutiert.

\subsection{1 Ähnlichkeiten und Unterschiede}

Obwohl das Phänomen der Vertragsforschung in diesem Kapitel aus der Perspektive von drei unterschiedlichen Theorierichtungen beleuchtet wurde, ergeben sich bei genauerer Analyse einige evidente Ähnlichkeiten. Sie sollen nachfolgend stichpunktartig festgehalten werden:

- Zunächst kann betont werden, dass die beiden institutionenökonomischen Forschungsansätze die Entscheidungen der Wirtschaftsakteure vor dem Hintergrund der Unvollständigkeit der Verträge und der wechselseitigen Abhängigkeit aufgrund von getätigten transaktionspezifischen Investitionen untersuchen. Es wird nicht von einer anonymen Marktbeziehung ausgegangen, sondern von Transaktionsbeziehungen, bei denen die gegenseitige Identität der Vertragsparteien eine besondere Rolle spielt. Die Analyse der FuE-Kooperationen im industrieökonomischen Forschungsprogramm erfolgt im Rahmen von Oligopolmodellen,

415 Kamien/Zang (2000), S. 1009. 
welche ebenfalls den Interdependenzen zwischen den Wirtschaftsakteuren Rechnung tragen.

- Alle Ansätze verweisen auf Gefahren, die entstehen, wenn eine externe Koordinationsform für die Abwicklung von FuE-Leistungen gewählt wird. Aus der Perspektive des Transaktionskostenansatzes können die Gefahren auf die Tendenz der Vertragsparteien zu opportunistischem Verhalten zurückgeführt werden. Der Property-Rights-Ansatz verweist auf die Bedeutung der Residualrechte, die zum einen die positive Wirkung der Verbesserung von Leistungsanreizen desjenigen Partners zur Folge haben, der diese Rechte behält, zum anderen jedoch Gefahren eines hold-up-Versuchs dieser Partei bei den Nachverhandlungen entstehen lassen. Im Rahmen der industrieökonomischen Modelle wird explizit auf die Problematik des Trittbrettfahrerverhaltens des Oligopolisten hingewiesen.

- Ferner kommen sowohl der Property-Rights-Ansatz als auch die industrieökonomischen Modelle zu dem Ergebnis, dass optimale Lösungen bei streng kooperativem Verhalten der Wirtschaftsakteure erreicht werden können. Zugleich wird jedoch argumentiert, dass trotz der Tatsache, dass ein perfekt abgestimmtes Verhalten der Wirtschaftsakteure im Hinblick auf die Gewinnmaximierung angestrebt werden sollte, sich die Wirtschaftsakteure gegen diese Lösung entscheiden können. Hart (1995) führt dies auf die Kondition der Unvollständigkeit der Verträge zurück. Kamien und Zang (2000) verweisen explizit auf das Phänomen der Transaktionskosten.

- Im Transaktionskostenansatz wird davon ausgegangen, dass Transaktionen, die hochspezifische Investitionen verlangen, intern abgewickelt werden. Im Modell von Kamien und Zang (2000) wird das Ergebnis formuliert, dass firmenspezifische Wissensressourcen dann erarbeitet werden, wenn sich ein Unternehmen gegen eine Zusammenarbeit mit einem unternehmensexternen Partner entscheidet. Ein Blick auf diese Resultate lässt die Vermutung zu, dass der internen Koordination von FuE-Leistungen dann der Vorzug gegeben wird, wenn es sich bei dem FuE-Vorhaben um die Erarbeitung hochspezifischer, unternehmensintern notwendiger Wissensressourcen handelt.

- Die Industrieökonomik betont die Rolle der internen Absorptionsfähigkeit als einer Voraussetzung für die Übernahme und effektive Anwendung des unternehmensextern erarbeiteten Wissens. Auch im Rahmen 
der Property-Rights-orientierten Analyse wurde argumentiert, dass die Problematik der "Nicht-Verifizierbarkeit" von Teilen der Vertragsmodalitäten - dabei insbesondere der durch den Auftragnehmer erbrachten FuE-Leistungen - für einen auf dem relevanten Technologiegebiet kompetenten Auftraggeber weniger ausgeprägt sein dürfte. Die Kondition der "Nicht-Verifizierbarkeit" dürfte sich besonders in der Gruppe der Unternehmen ohne FuE-Kompetenz transaktionshemmend auswirken. Im Hinblick auf die Argumentationsstruktur beider Forschungsrichtungen kann folglich die Vermutung aufgestellt werden, dass das Fehlen interner FuEKompetenz die Entscheidung für eine Auftragsvergabe verhindert. Dem Instrument der Vertragsforschung dürfte eine besondere Rolle als Mittel der komplementären Wissensbeschaffung zukommen.

Zuletzt soll auf ein spezielles Ergebnis der Industrieökonomik im Hinblick auf die Bedeutung von Externalitäten der FuE-Tätigkeit hingewiesen werden. Wird davon ausgegangen, dass die Spillover-Problematik von den Wirtschaftsakteuren in einer Entscheidungssituation explizit berücksichtigt wird, dann ist im Sinne der industrieökonomischen Modelle davon auszugehen, dass in einem Technologieumfeld, welches durch erhebliche Spilloverraten gekennzeichnet ist, die Unternehmen mehr Anreize haben, eine Zusammenarbeit mit anderen Wirtschaftsakteuren einzugehen, da sie in diesem Falle die SpilloverEffekte ihrer FuE-Tätigkeit internalisieren können. Während folglich aus der Perspektive des TKAs argumentiert werden muss, dass die Vertragsparteien ihre Wissensvorsprünge voreinander schützen sollten, kann im Rahmen der industrieökonomischen Modelle der FuE-Kooperation gezeigt werden, dass bei ohnehin vorhandenen erheblichen Spillovereffekten eine Entscheidung für die Zusammenarbeit mit unternehmensexternen Partnern und für eine weitere vollständige - Offenlegung aller FuE-relevanten Informationen die gewinnoptimale Alternative darstellt.

\subsubsection{Zusammenführung der Hypothesen}

Während in den einzelnen Abschnitten dieses Kapitels das Phänomen der Organisation der FuE-Tätigkeit im Wirtschaftssektor bei expliziter Berücksichtigung der facettenreichen Argumentationsstruktur einzelner Ansätze differenziert durchleuchtet wurde, was die Ableitung mehrerer Hypothesen über die Bestimmungsfaktoren der Vertragsforschung ermöglichte, sollen in diesem Abschnitt die bisherigen Ausführungen auf einige zentrale Erkenntnisse verdichtet 
werden. Die im Laufe der Untersuchung formulierten Hypothesen seien nachfolgend nochmals aufgelistet:

Hypothese 1 Eine hohe Einschätzung der Effektivität von Mechanismen zum Schutz eigenen innovationsrelevanten Wissens begünstigt die Entscheidungen für die Vertragsforschung.

Hypothese 2 Das in Kooperationsbeziehungen aufgebaute Vertrauen zum Transaktionspartner begünstigt die Entscheidung für Vertragsforschung.

Hypothese 3 Auftragnehmer sind unter denjenigen Unternehmen zu erwarten, die über materielle (Ausrüstungsgegenstände) und immaterielle (distinktive Kompetenzen) Ressourcen verfügen, welche nicht erst als transaktionsspezifische Investitionen aufgebracht werden müssen.

Hypothese 4 Die Vertragsforschung stellt ein kostengünstiges institutionelles Arrangement dar im Kontext von Auslastungsschwankungen und Engpassüberwindung.

Hypothese 5 Die Vertragsforschung spielt für die Unternehmen der Spitzentechnologie eine wichtige Rolle als ein Instrument der Sondierung neuer technologischer Entwicklungen.

Hypothese 6 Die Auftragsvergabe wird in der Gruppe der Großunternehmen als ein Instrument zur Bewältigung interner Koordinationsprobleme eingesetzt.

Hypothese 7 Interne FuE-Kompetenz begünstigt die Entscheidung für die Auftragsvergabe.

Hypothese 8 Vertragsforschungsbeziehungen entstehen als Folge einer Ausgliederung von FuE-Gesellschaften.

Hypothese 9 Die Vertragsforschung wird bei einer großen Bedeutung des Humankapitals unternehmensexterner Partner erwartet.

Hypothese 10 Für die Auftragsvergabe stellt die interne Absorptionsfähigkeit eine notwendige Bedingung dar.

Ein Blick auf die einzelnen Hypothesen ermöglicht sodann eine Unterscheidung von zwei Gruppen von Motiven, die Entscheidungen für eine Vertragsvergabe begünstigen. Die erste Gruppe von Motiven betont die Bedeutung der 
im technologischen Umfeld einer Unternehmung zugänglichen innovationsrelevanten Ressourcen und Kompetenzen (Hypothesen 3, 5 und 9), d.h. - in der Terminologie der Innovationsforschung - der technologischen Möglichkeiten. Unternehmen greifen demnach auf das Instrument der Vertragsforschung insbesondere dann zurück, wenn sie Zugriff auf Kompetenzen der unternehmensexternen Partner erhalten oder sich einen Einblick in neuere, auf dem relevanten Gebiet stattfindende technologische Entwicklungen verschaffen wollen. Da die unternehmensintern vorhandene Absorptionsfähigkeit eine Voraussetzung für die Übernahme des extern erarbeiteten Wissens bildet (vgl. Hypothesen 7 und 10), kann davon ausgegangen werden, dass diese ressourcenbzw. kompetenzorientierte Auftragsvergabe für FuE-aktive Unternehmen eine besondere Rolle spielt.

Der Zugriff auf unternehmensextern bereits vorhandene Kompetenzen ermöglicht sodann eine Reduzierung der Entwicklungszeiten; ein Faktor, dem angesichts der Verkürzung der Lebenszyklen von Produkten und Technologien eine herausragende Rolle zukommt. Allerdings ist in diesem Falle die Entscheidung für die Auftragsvergabe mit dem Verzicht auf einen eigenständigen Aufbau des unternehmensintern benötigten Wissens verbunden, wodurch Lernkurveneffekte nicht erzielt werden. Den kumulativen Charakter der FuE-Tätigkeit unterstellt, ist in diesem Falle von einer zunehmenden Abhängigkeit von unternehmensexternen Partnern auszugehen. Dies kann wiederum die nachfolgende Entscheidung für eine vertikale Integration begünstigen.

Bei dieser ressourcen- bzw. kompetenzorientierten Auftragsvergabe kommt es folglich zu einer Abwägung zwischen den Vorteilen eines schnellen Zugriffs auf die im technologischen Umfeld vorhandene FuE-Kompetenz und den Nachteilen, die sich aus dem Verzicht auf einen eigenständigen Aufbau des unternehmensintern benötigten Wissens ergeben.

Die zweite Gruppe von Motiven betont die Kostenvorteile der marktlichen Abwicklung von FuE-Leistungen. So kann Vertragsforschung dann eingesetzt werden, wenn es zu periodenabhängigen Schwankungen in der Auslastung unternehmensinterner FuE-Kapazitäten kommt (Hypothese 4). Der Vorteil externer Koordinationsformen begründet sich in diesem Falle in der erhöhten Flexibilität bei der Planung von FuE-Projekten. Kostenvorteile können ebenfalls durch Ausgliederung von periphären FuE-Kapazitäten erzielt werden (Hypothese 8). Erbringt die ausgegliederte Gesellschaft auch FuE-Leistungen für Dritte, dann können fixe Kosten der Vorhaltung gewisser Kapazitäten auf mehrere Projekte und Wirtschaftsakteure verteilt werden. Schließlich verweist die Hypothese 6 auf die Möglichkeiten der Senkung von 
Koordinationskosten in Großunternehmen durch den externen Bezug von spezifizierten FuE-Leistungen.

Die obige Analyse verdeutlicht zugleich, dass bei der Untersuchung des Phänomens der Vertragsforschung auf die in Kapitel 3 erläuterten Konzepte der Innovationsökonomik zurückgegriffen werden kann. Die Bedeutung der Kompetenzen und Ressourcen der unternehmensexternen Partner wird in der innovationsökonomischen Literatur unter Zuhilfenahme des Konzepts der technologischen Möglichkeiten konzeptualisiert und meist unter Anwendung von einzelwirtschaftlichen Angaben zur Nutzung von externen Wissensquellen operationalisiert. Dieser Methodologie wird in der nachfolgenden empirischen Analyse gefolgt. Die Problematik der Externalitäten der FuE-Tätigkeit wird in der innovationsökonomischen Literatur ebenfalls stark betont. Zur Operationalisierung des Phänomens der unvollständigen Appropriierungsmöglichkeiten wird meist auf die von Levin et al. (1987) etablierte Methodologie der Messung der Effektivität von Mechanismen zum Schutz des Wissens- und Technikvorsprungs abgestellt. Auch diese Operationalisierungsmethode wird in den nachfolgenden Schätzungen verwendet. 


\section{Kapitel 5}

\section{Determinanten der Vertragsforschung in der Empirie}

Die Ausführungen dieses Kapitels gelten der empirischen Untersuchung des Phänomens der Vertragsforschung. Im Mittelpunkt der Analyse steht dabei die Überprüfung der im theoretischen Teil der Arbeit formulierten Hypothesen über die Determinanten der Vertragsforschungsaktivitäten.

Erste Aufschlüsse über die Gültigkeit einzelner Hypothesen können bereits aus der Analyse vorliegender Studien gewonnen werden. Daher sollen in Abschnitt 5.2 die in der empirischen Literatur bisher gewonnenen Erkenntnisse über den deutschen FuE-Dienstleistungsmarkt synoptisch erläutert werden.

In Abschnitten 5.3 und 5.4 werden die Schätzungen der Determinanten der Vertragsforschung anhand der im Rahmen des Mannheimer Innovationspanels erhobenen Unternehmensdaten vorgenommen. Die hierbei gewonnenen Ergebnisse werden den Resultaten anderer ökonometrischer Studien gegenübergestellt. Sämtliche in der einschlägigen Literatur vorzufindenden ökonometrischen Beiträge basieren auf Daten zur Forschungstätigkeit von Unternehmen aus anderen europäischen Ländern und aus den Vereinigten Staaten, was eine vergleichende Analyse und zugleich eine Identifizierung der länderübergreifend geltenden Zusammenhänge ermöglicht.

Eine Diskussion der zentralen Ergebnisse des empirischen Teils der Arbeit in Abschnitt 5.5 schließt die Ausführungen dieses Kapitels ab. 


\subsection{Anmerkungen zu Datenquellen}

Bevor die empirische Analyse des Phänomens der Vertragsforschung erfolgt, sollen zunächst die Datenquellen dargestellt werden, denen Informationen zur Forschungstätigkeit im Wirtschaftssektor Deutschlands entnommen werden können und auf denen die nachfolgend zu erläuternden Studien über den deutschen FuE-Dienstleistungsmarkt basieren. Der Schwerpunkt liegt dabei auf der Erläuterung besonderer Fragestellungen der einzelnen Erhebungen, der Darlegung der Art der gewonnen Daten und schließlich der Begründung der Wahl des Mannheimer Innovationspanels (MIP) als Datenbasis für die vorliegende Studie (Abschnitt 5.1.1). Anschließend wird in Abschnitt 5.1.2 das Erhebungsdesign des MIP näher erläutert.

\subsubsection{Die deutsche FuE-Statistik}

Das System der FuE-Statistik ist in Deutschland sektoral organisiert. ${ }^{416}$ Das Statistische Bundesamt ermittelt Daten zu FuE-Aufwendungen und zum FuEPersonal der Hochschulen, des Staatssektors und der privaten Organisationen ohne Erwerbszweck. Die Daten zur FuE-Tätigkeit im Wirtschaftssektor werden in Selbstverantwortung der Wirtschaft erhoben. Diese Aufgabe liegt seit über 40 Jahren bei einer durch die Wirtschaft getragenen Institution zur Förderung der Wissenschaft, dem Stifterverband für die Deutsche Wissenschaft. Mit der Aufgabe der Durchführung der Datenermittlung hat der Stifterverband seine Tochter, die Wissenschaftsstatistik $\mathrm{GmbH}$, beauftragt.

Die Daten werden in ungeraden Jahren auf Basis einer Vollerhebung und in geraden Jahren auf Basis einer Kurzerhebung ermittelt. Die Grundgesamtheit der jeweiligen Erhebung für die ungeraden Jahre wird aus „alle[n] bekannten FuE-affinen Unternehmen " ${ }^{417}$ des Wirtschaftssektors gebildet, d.h. aus Wirtschaftseinheiten, von denen angenommen wird, dass sie positive FuEAufwendungen aufweisen. Die Erhebung erfolgt in Übereinstimmung mit den Empfehlungen des international abgestimmten Regelwerkes der OECD, des Frascati-Handbuchs.

Die von der Wissenschaftsstatistik $\mathrm{GmbH}$ ermittelten Daten zur Forschungstätigkeit im Wirtschaftssektor waren bis zum Erhebungsjahr 1999 allei-

416 Vgl. Grenzmann (2004), S. 8 f.

417 Vgl. Legler/Grenzmann/Marquandt (2006), S. 9. 
nige Datenbasis für die nationale und internationale Berichterstattung. ${ }^{418}$ Seit 1999 ermittelt das Statistische Bundesamt im Rahmen der Kostenstrukturerhebung im Verarbeitenden Gewerbe, Bergbau sowie in der Gewinnung von Steinen und Erden zusätzliche Angaben zur innerbetrieblichen Forschung und Entwicklung. ${ }^{419}$ Im Unterschied zu den Erhebungen der Wissenschaftsstatistik besteht bei der Kostenstrukturerhebung eine Auskunftspflicht.

Angaben zur Forschungs- und Entwicklungstätigkeit im Wirtschaftssektor in Deutschland können auch aus wiederkehrenden Erhebungen weiterer Institutionen entnommen werden: ${ }^{420}$

- Seit 1993 führt das Zentrum für Europäische Wirtschaftsforschung (ZEW) - in Zusammenarbeit mit dem infas Institut für angewandte Sozialwissenschaft - systematische Erhebungen zum Innovationsverhalten der deutschen Wirtschaft durch. Im sogenannten Mannheimer Innovationspanel werden Forschungs- und Entwicklungsaufwendungen der Unternehmen als Teil der Innovationsaufwendungen in Übereinstimmung mit den Abgrenzungen des Frascati-Handbuchs in den Erhebungen explizit berücksichtigt. ${ }^{421}$

- Das ifo Institut für Wirtschaftsforschung führt seit 1979 einen sogenannten Innovationstest durch, der sich methodisch aus zwei Teilen zusammensetzt: Im Rahmen des ifo-Konjunkturtests werden zunächst allgemeine Fragen zur Innovationstätigkeit gestellt. Aus dem Berichtskreis des ifo-Konjunkturtests werden anschließend vorwiegend diejenigen Unternehmen um Ausfüllung eines detaillierteren Fragebogens des Innovationstests gebeten, die positive Innovationsaktivitäten verzeichnen. ${ }^{422}$

- Die Kreditanstalt für Wiederaufbau (KfW) führt seit 2003 eine repräsentative Befragung aller mittelständischen Unternehmen in Deutschland durch. Im Rahmen des sogenannten KfW-Mittelstandspanels liegt

$418 \mathrm{Vgl}$. Haug/Revermann (2003), S. 1130.

419 Vgl. Statistisches Bundesamt (2005).

420 Neben den nachfolgend aufgelisteten Erhebungen nennen Legler et al. (2006) auch die Studien von Euronorm (früher: Forschungsagentur Berlin), in denen regelmäßig im Auftrag des BMWi/BMWA das FuE-Verhalten in den neuen Bundesländern - als begleitende Untersuchung der FuE-Förderung - verfolgt wird. Vgl. Legler/Grenzmann/Marquandt (2006), S. $7 \mathrm{f}$.

421 Vgl. Janz/Ebling/Gottschalk/Peters/Schmidt (2003), S. 14.

422 Vgl. Penzkofer (2004), S. 149 ff. 
der Schwerpunkt auf der Erhebung von Daten zur Unternehmensentwicklung, Investitionstätigkeit und Finanazierungsstuktur der deutschen KMU. In den Befragungen aus den Jahren 2003 und 2005 wurden auch Informationen zum Innovationsverhalten der KMU ermittelt. ${ }^{423}$

- Eckdaten zur Forschungs- und Entwicklungstätigkeit werden in unregelmäßigen Abständen vom Institut für Arbeitsmarkt- und Berufsforschung (IAB) im Rahmen des IAB-Betriebspanels erhoben. Das IAB-Betriebspanel ist eine jährliche Arbeitgeberbefragung, die seit 1993 in Westdeutschland und seit 1996 auch in den neuen Bundesländern durchgeführt wird. Allgemeine Fragen zur Forschungs- und Entwicklungstätigkeit wurden in den Erhebungen der Jahre 1993, 1998 und 2004 gestellt. $^{424}$

Aufgrund der unterschiedlichen Schwerpunktlegung einzelner Studien (Beschäftigungstrends beim IAB-Betriebspanel, Innovationsgeschehen beim MIP, FuE-Tätigkeit beim Stifterverband) unterscheiden sich die einzelnen Datenquellen stark voneinander im Hinblick auf die Verfügbarkeit von innovationsrelevanten Daten. In der Kostenstrukturerhebung des Statistischen Bundesamtes und im IAB-Betriebspanel wird grundsätzlich nicht zwischen interner und externer Forschung und Entwicklung unterschieden. Daher können die Ergebnisse dieser Erhebungen nicht als Datengrundlage für die nachfolgenden Untersuchungen herangezogen werden. Der KfW-Mittelstandspanel beschränkt sich auf die Gruppe der KMU. Da ein Einfluss der Unternehmensgröße auf Entscheidungen im Bereich der Vertragsforschung im Lichte der vorliegenden Studien nicht ausgeschlossen werden kann, muss ebenfalls auf einen Rückgriff auf die KfW-Daten verzichtet werden.

Der Stifterverband für die Deutsche Wissenschaft stellt zwar statistische Daten zur innerbetrieblichen FuE in der notwendigen Ausdifferenzierung zur Verfügung. Allerdings beschränken sich die Erhebungen der Wissenschaftsstatistik im Wesentlichen auf die Ermittlung von FuE-Daten, so dass die für die ökonometrische Analyse relevanten Informationen, wie zum Beispiel Angaben $\mathrm{zu}$ Wettbewerbsbedingungen, Innovationshemmnissen oder Aneignungsstrategien, nicht zugänglich sind. Die Daten des Stifterverbandes sowie des ifo-Innovationstests wurden zudem bereits in einer umfangreichen Studie über

$423 \mathrm{Vgl}$. KfW-Bankengruppe (2005), S. $12 \mathrm{f}$.

${ }^{424}$ Eine Frage zur FuE-Tätigkeit wurde auch in dem Zusatzfragebogen (für neue Betriebe) der Erhebung von 1994 gestellt. 
den FuE-Dienstleistungsmarkt in Deutschland ausgewertet. ${ }^{425}$ Die Ergebnisse dieser Studie werden in Abschnitten 5.2.2 und 5.2.3 näher erläutert. Auf die Datensätze des Mannheimer Innovationspanels wurde in den bisherigen Studien zum FuE-Dienstleistungsmarkt in Deutschland nicht zurückgegriffen. Daher werden bei der Schätzung der Determinanten der Vertragsforschung in Abschnitten 5.3 und 5.4 die Daten des MIP zugrunde gelegt.

\subsubsection{Das Mannheimer Innovationspanel (MIP)}

Beim Mannheimer Innovationspanel handelt es sich um systernatische Erhebungen zum Innovationsverhalten der deutschen Wirtschaft, die vom Zentrum für Europäische Wirtschaftsforschung (ZEW) in Zusammenarbeit mit dem infas Institut für angewandte Sozialwissenschaft - im Auftrag des Bundesministeriums für Bildung und Forschung - seit 1993 durchgeführt werden. Die Erhebungen aus den Jahren 1993, 1997 und 2001 bildeten den deutschen Beitrag zu den von Eurostat koordinierten europaweiten Innovationserhebungen (Community Innovation Surveys, CIS I bis III). ${ }^{426}$

Die Erhebungsmethodik des Mannheimer Innovationspanels basiert auf dem Oslo-Handbuch (Oslo Manual), in welchem Empfehlungen hinsichtlich der Richtlinien zur Erhebung und Interpretation von technologischen Innovationsdaten seitens OECD und Eurostat gegeben werden. ${ }^{427}$ Forschungs- und Entwicklungsaufwendungen der Unternehmen verstanden als Teil der Innovationsaufwendungen ${ }^{428}$ werden in Übereinstimmung mit den Abgrenzungen des Frascati-Handbuchs in den Erhebungen explizit berücksichtigt.

Die Erhebungen des MIP sind als Panelstichproben konzipiert. ${ }^{429}$ Aus der Zielgrundgesamtheit, bestehend aus rechtlich selbstständigen Unternehmen

\footnotetext{
$425 \mathrm{Vgl}$. Koschatzky/Reinhard/Grenzmann (2003).

426 Vgl. Janz/Ebling/Gottschalk/Peters (2002), Janz/Ebling/Gottschalk/Peters/Schmidt (2003), Janz/Ebling/Gottschalk/Niggemann (2001).

427 Vgl. OECD/Eurostat (1997).

$428 \mathrm{Zu}$ den Innovationsaufwendungen gehören nach Abgrenzungen des Oslo-Handbuchs neben den FuE-Aufwendungen: (1) Ausgaben für externes Wissen (disembodied technology and know-how) wie beispielweise Patente und Lizenzen, (2) Ausgaben für Maschinen und Sachmittel (embodied technology), (3) Ausgaben für Konstruktion, Produktgestaltung, Prototypen und Vorbereitungen für die Produktion, (4) Weiterbildungsausgaben und (5) Ausgaben für die Markteinführung von Produkten, vgl. OECD/Eurostat (1997), S. 87.

${ }^{429}$ Vgl. Janz/Ebling/Gottschalk/Peters/Schmidt (2003), S. 12.
} 
mit mindestens 5 Beschäftigten, wird eine geschichtete Zufallsstichprobe gezogen. ${ }^{430}$ In jedem Erhebungsjahr werden die gleichen statistischen Einheiten (Unternehmen) befragt. Die Stichprobe wird jedoch zweijährlich um eine geschichtete Zufallstichprobe vorwiegend aus neu gegründeten Unternehmen ergänzt und jährlich um Unternehmenszusammenschlüsse bzw. -schließungen bereinigt. Ostdeutsche Unternehmen haben eine höhere Auswahlwahrscheinlichkeit. ${ }^{431}$ Die Liste der regelmäßig erhobenen Variablen (Strukturvariablen der Unternehmen und zentrale Innovationsvariablen) wird bei der jeweiligen Erhebung - unter Berücksichtigung spezieller Forschungsschwerpunkte - um eine Vielzahl von detaillierten Fragestellungen ergänzt. Dadurch unterscheiden sich die einzelnen Wellen des MIP zum Teil stark im Hinblick auf die Verfügbarkeit von für die nachfolgenden Schätzungen relevanten Daten.

Deshalb sind als Grundlage für die folgende Analyse zwei Wellen des Mannheimer Innovationspanels ausgewählt worden. Zum einen ist dies die erste MIPWelle aus dem Jahre 1993, die bei den Schätzungen in Abschnitt 5.3 zugrunde gelegt wird. Der Vorteil dieses Datensatzes begründet sich schwerpunktmäßig in der Zugänglichkeit von Angaben zur Durchführung von FuE-Aufträgen. Diese Daten wurden nur im ersten Erhebungsjahr ermittelt. In späteren Fragebögen sind lediglich Fragen zur externen FuE gestellt worden, die zwar eine Erfassung des Phänomens der Auftragsvergabe ermöglichen, aber keine Aufschlüsse über die Gruppe der FuE-Dienstleister aus dem Wirtschaftssektor liefern. Da aufgrund der Schwierigkeiten der Datenermittlung gerade diese letztere Gruppe bisher wenig untersucht wurde, ist es sinnvoll, auf den Datensatz der ersten Erhebungswelle zurückzugreifen, um grundlegende Erkenntnisse über die Anbieter der FuE-Dienstleistungen aus dem Wirtschaftssektor zu gewinnen. Darüber hinaus liefert dieser Datensatz Angaben zur Nutzung von verschiedenen Formen des Erwerbs bzw. der Weitergabe neuen technologischen Wissens durch Unternehmen. Dies lässt Schätzungen über die Verbreitung der hybriden und kooperativen Koordinationsformen für FuE-Leistungen zu. Schließlich bietet dieser Datensatz eine Vielzahl von detaillierten Angaben zu Innovationshemmnissen, zur Wettbewerbssituation sowie Effektivität der Mechanismen zum Schutz des innovationsrelevanten Wissens und zur Bedeutung von unternehmensexternen Wissensquellen, die zum Teil ebenfalls in späteren Fragebögen nicht berücksichtigt wurden.

430 Die Schichtung erfolgt nach Branchen, Betriebsgrößenklassen und Regionen (alte bzw. neue Bundesländer); für Einzelheiten der Stichprobenziehung vgl. Janz/Ebling/Gottschalk/Peters (2002).

431 Vgl. Janz/Ebling/Gottschalk/Peters/Schmidt (2003), S. 12. 
Zum anderen wird in Abschnitt 5.4 die letzte für externe Nutzer zugängliche Welle des MIP aus dem Jahre 2003 zugrunde gelegt. Dieser Datensatz liefert Daten zu externen FuE-Aufwendungen der Unternehmen und erlaubt damit eine Schätzung der Determinanten der Auftragsvergabe. Ein Vergleich der Ergebnisse der Schätzungen anhand der ersten und letzten MIP-Welle ermöglicht die Identifizierung der Bestimmungsfaktoren der Vertragsforschung, die unabhängig vom Zeitpunkt der Betrachtung eine wichtige Rolle spielen.

Für externe Nutzer stehen die Daten des MIP in anonymisierter Form für eine nicht kommerzielle Grundlagenforschung zur Verfügung. Für die nachfolgenden Untersuchungen ist jedoch auf die Originaldaten zurückgegriffen worden, so dass Probleme, die aufgrund der Nutzung von anonymisierten Datensätzen entstehen, diese Arbeit nicht tangieren.

\subsection{Ergebnisse bisheriger empirischer Studien}

In diesem Abschnitt sollen die wichtigsten Befunde bereits vorliegender empirischer Studien zum FuE-Dienstleistungsmarkt in Deutschland erläutert werden. Einige als zentral betrachtete Studien sind dabei gewählt worden, welche eine ausdifferenzierte Betrachtung des Phänomens der Vertragsforschung erlauben. ${ }^{432}$ In Abschnitt 5.2.1 werden zunächst die wichtigsten Ergebnisse von zwei Evaluierungen der Fördermaßnahme des Bundesministeriums für Bildung und Forschung „Externe Vertragsforschung“ dargelegt. Die Befunde dieser Studien ermöglichen Rückschlüsse über die Nachfrager von FuE-Dienstleistungen aus der Gruppe der Klein- und Mittelunternehmen. In den Abschnitten 5.2.2 und 5.2.3 werden die zentralen Ergebnisse der neuesten und umfangreichsten Untersuchung zum FuE-Dienstleistungsmarkt in Deutschland aus dem Jahre 2003 erläutert. Abschnitt 5.2.4 fasst die Ergebnisse einer Studie über die ostdeutschen Vertragsforschungsanbieter, die sogenannten „externen Industrieforschungseinrichtungen", zusammen.

432 Auf eine gesonderte Darlegung der Ergebnisse der Studie von Rötlılingshöfer (1972) wird verzichtet, da sie sich schwerpunktmäßig mit der Bestandsaufnahme der Vertragsforschungsaktivitäten zum Ende der 60er Jahre beschäftigt. Auf einige in dieser Studie formulierte Tendenzaussagen wird in Abschnitt 5.2.5 hingewiesen. Auch die Befunde der Studien zu Vertragsforschungsaktivitäten in der Pharmaindustrie bzw. dem Biotechnologiesektor Deutschlands werden als Fallstudien einzelner Branchen vor dem Hintergrund des breiter angelegten Forschungsinteresses der vorliegenden Arbeit nicht gesondert analysiert. Vgl. hierzu Dillmann (1996), Dillmann (1997), Reiß/Hüsing (1992). 


\subsubsection{Evaluierung der Fördermaßnahme „Externe Ver- tragsforschung"6}

Die Fördermaßnahme „Externe Vertragsforschung“433 war Teil eines Gesamtkonzepts der Bundesregierung zur Förderung der Innovationstätigkeit von kleinen und mittleren Unternehmen und gründete sich auf die Einschätzung, dass KMUs „keinen ständigen Bedarf an Forschung und Entwicklung haben oder ihnen sachliche, personelle oder finanzielle Voraussetzungen zur Durchführung solcher Arbeiten fehlen “434. Durch die Bereitstellung von Fördermitteln sollten die Zugangsmöglichkeiten der Klein- und Mittelbetriebe zu Forschungsund technologischen Dienstleistungen verbessert und ihre Innovationskraft gestärkt werden. Im Rahmen dieser Maßnahme konnten Unternehmen mit einem Jahresumsatz von bis zu 200 Mio $\mathrm{DM}^{435}$, die externe Partner mit der Durchführung von bestimmten FuE-Leistungen beauftragt haben, einen Zuschuss von $30 \%$ der Projektkosten erhalten. Als Auftragnehmer konnten zunächst sowohl öffentlich finanzierte Einrichtungen als auch private Forschungsinstitute, Ingenieurbüros oder FuE-betreibende Unternehmen fungieren; nach einer Richtlinienänderung wurden Forschungsaufträge an produzierende Unternehmen als nicht mehr förderfähig deklariert. ${ }^{436}$ Eine Zwischenevaluation dieser Fördermaßnahme wurde 1982 vom Fraunhofer-Institut für Systemtechnik und Innovationsforschung (ISI) und der Technologie-TransferStelle der Technischen Universität Berlin durchgeführt. ${ }^{437}$

Die statistische Grundauswertung von 909 Anträgen zeigte, dass über die Hälfte der geförderten Vorhaben auf Antragssteller aus den Branchen Maschinenbau, Elektrotechnik und chemische Industrie entfielen. Als Auftragnehmer fungierten vorwiegend Akteure der folgenden Wirtschaftszweige: Wissenschaft und Forschung (32\%), Dienstleistungen für Unternehmen (32\%) sowie Unternehmen des produzierenden Gewerbes (25\%), darunter insbesondere die Branchen Maschinenbau und Elektrotechnik. ${ }^{438}$

433 Nach der Empfehlung der Zwischenevaluierung der Fördermaßnahme wurde später die Bezeichnung „Auftragsforschung und Entwicklung“ verwendet.

434 Allesch/Fiedler/Martin (1983), S. 3.

435 Im Jahre 1984 wurde diese Obergrenze auf 500 Mio DM angehoben; zu weiteren Förderrichtlinien vgl. Fraunhofer-ISI/Prognos AG (1991), S. 222.

436 Vgl. Allesch/Fiedler/Martin (1983), S. 3, Fraunhofer-ISI/Prognos AG (1991), S. 222.

437 Vgl. Allesch/Fiedler/Martin (1983).

438 Vgl. Allesch/Fiedler/Martin (1983), S. 5 ff. sowie Tab. 20 und Tab. 29 im Tabellenanhang. 
Eine schriftliche und persönliche Befragung der Zuwendungsempfänger und der Auftragnehmer ermöglichte zudem die folgenden Schlussfolgerungen:

Die geförderten Unternehmen verfügten fast alle über eigene FuE-Kapazitäten und waren vergleichsweise forschungsintensiv: Sie wendeten im Jahre $1981 \mathrm{im}$ Durchschnitt $6 \%$ ihres Umsatzes für Forschung und Entwicklung auf. Fast alle (98\%) zählten zur Gruppe der Innovatoren, d.h. gaben an, in den vergangenen fünf Jahren neue oder verbesserte Produkte und/oder Verfahren eingeführt zu haben. ${ }^{439}$ Die Analyse zeigte folglich, dass es sich bei den Zuwendungsempfängern mehrheitlich um Unternehmen handelte, die über eigene FuEKapazitäten verfügten. Das Instrument der Vertragsforschung wurde von ihnen nicht angesichts eines Mangels an eigenen FuE-Kapazitäten eingesetzt, sondern zur Verstärkung vorhandener Innovationskompetenz herangezogen. Bereits in der Gruppe der KMU konnten folglich Hinweise auf Komplementarität der externen und internen Beschaffung des innovationsrelevanten Wissens gefunden werden.

Ferner wurden die Zuwendungsempfänger nach den Gründen für die Vergabe von Entwicklungsaufträgen gefragt. Es zeigte sich hierbei die in Tab. 5.1 angebende Rangfolge von Motiven der Auftragsvergabe.

Tabelle 5.1: Gründe für die Vergabe von Entwicklungsaufträgen

\begin{tabular}{lc}
\hline \hline Motiv & $\begin{array}{c}\text { Häufigkeit } \\
\text { der Nennung }\end{array}$ \\
\hline spezielles Know-how nicht vorhanden & $58,0 \%$ \\
apparative Einrichtungen nicht vorhanden & $46,9 \%$ \\
FuE-Personal zeitlich ausgelastet & $42,0 \%$ \\
Vergabe nach außen kostengünstiger & $15,6 \%$ \\
kein FuE-Personal & $10,7 \%$ \\
\hline \hline
\end{tabular}

Anmerkung: Mehrfachnennungen möglich.

Quelle: Allesch/Fiedler/Martin (1983), S. 59.

Es wurde deutlich, dass Unternehmen, die über eigene FuE-Kapazitäten verfügten, dennoch in ihrer Innovationsstrategie auf unternehmensexternes

$439 \mathrm{Vgl}$. Allesch/Fiedler/Martin (1983), S. $42 \mathrm{ff}$. 
Know-how sowie apparative Vorrichtungen unternehmensexterner Partner angewiesen waren. Es wurden folglich Hinweise auf die Relevanz des Zugriffs auf komplementäre Ressourcen unternehmensexterner Partner gefunden. So wiesen die Autoren der Zwischenbilanz darauf hin, dass beispielweise Unternehmen aus dem Bereich Maschinenbau im Rahmen ihrer Innovationsstrategie auf das Instrument der Vertragsforschung zuzugreifen schienen, um „den technologischen Fortschritt in der Elektrotechnik und Mikroelektronik für das Produktionsprogramm nutzbar zu machen" ${ }^{\star 440}$.

Die Analyse der Motive der Auftragsvergabe lieferte ferner einen Hinweis auf die Gültigkeit der Hypothese über die Bedeutung der Vertragsforschung bei Schwankungen in der Auslastung der internen FuE-Einrichtungen. Für $42 \%$ der befragten Unternehmen stellte die zeitweise volle Auslastung des FuEPersonals einen der Gründe für die Auftragsvergabe dar. Auffällig war zudem die relativ geringe Bedeutung des Kostenfaktors: Nur 15,6\% der Unternehmen nannten diesen Aspekt als eines der relevanten Motive. Schließlich bleibt zu bemerken, dass über $10 \%$ der Unternehmen auf das Instrument der Vertragsforschung angesichts offensichtlich fehlender interner FuE-Kapazitäten zugriff.

Angaben der Unternehmen zur Beurteilung der Zusammenarbeit mit den Auftragnehmern ermöglichte zudem eine Analyse der während der Abwicklungsphase entstehenden Probleme. So gaben $14 \%$ der Unternehmen an, dass die veranschlagten Kosten, und $40 \%$, dass der Zeitplan für die Erbringung der FuE-Leistung überschritten wurden. Ferner war aus der Sicht von $43,5 \%$ der Zuwendungsempfänger eine regelmäßige Abstimmung bei der Abwicklung des Vorhabens notwendig; nur bei $1,5 \%$ der Unternehmen fand keine Zwischenabstimmung in der Vertragsabwicklungsphase statt. ${ }^{441}$

Im Auftrag des Bundesministeriums für Forschung und Technologie wurde 1991 eine weitere Evaluation der Fördermaßnahme durchgeführt. Hierbei bestätigte sich die Beobachtung einer tendenziell stärkeren Innovationsorientierung derjenigen Unternehmen, die das Instrument der Vertragsforschung genutzt haben: Die geförderten Unternehmen beschäftigten im Schnitt mehr FuE-Personal, nahmen deutlich häufiger neue Produkte in ihr Produktionsprogramm auf und sahen sich öfter steigenden Entwicklungsaufwendungen und kurzen Produktlebenszyklen gegenüber als Unternehmen der Vergleichsgruppe. ${ }^{442}$ Erneut zeigte sich zudem die Bedeutung des Zugangs zu distinktiven

440 Allesch/Fiedler/Martin (1983), S. 51.

$441 \mathrm{Vgl}$. Allesch/Fiedler/Martin (1983), S. 60.

442 Vgl. Fraunhofer-ISI/Prognos AG (1991), S. 228 f. 
Kompetenzen unternehmensexterner Partner als zentrales Motiv der Auftragsvergabe: Für $74 \%$ der Unternehmen war die Tatsache, dass spezielles Wissen intern nicht vorhanden ist, ein wichtiger bzw. sehr wichtiger Grund für den externen Bezug der FuE-Leistung. Bei der Untersuchung der Kriterien für die Partnerauswahl wurde auch deutlich, dass die auftraggebenden Unternehmen bei den externen Partnern vorwiegend technische Kompetenz und hohe Anwendungsnähe suchten. ${ }^{443}$ Insgesamt bestätigte sich der stark komplementäre Charakter der Vertragsforschung: Die geförderten Aufträge bezogen sich in $57 \%$ der Fälle auf Teilaufgaben von Gesamtprojekten. Daher kamen die Autoren der Studie zu dem Ergebnis, dass die geförderten FuE-Aufträge sehr oft ein integraler Teil der normalen FuE-Tätigkeit der betreffenden Unternehmen waren..$^{444}$

\subsubsection{Sonderauswertung der Wissenschaftsstatistik}

Im Jahre 2001 wurde die Wissenschaftsstatistik $\mathrm{GmbH}$ vom Bundesministerium für Bildung und Forschung (BMBF) beauftragt, eine Sonderauswertung der externen FuE-Aufwendungen im Wirtschaftssektor zu erstellen. Grundlage für diese Auswertung bildeten die von der Wissenschaftsstatistik erhobenen Daten zur FuE-Tätigkeit im Wirtschaftssektor Deutschlands. Die Ergebnisse dieser Sonderauswertung werden nachfolgend eingehender erläutert, da sie im Unterschied zu weiteren Studien sowie dem methodologischen Ansatz dieser Arbeit - nicht die Anteile der auftragserteilenden Unternehmen ermitteln, sondern Aussagen über die absoluten Volumina der externen FuE-Aufwendungen in Abhängigkeit von Strukturmerkmalen der Unternehmen zulassen.

In der Auswertung nach Betriebsgrößenklassen ließen die Daten der Wissenschaftsstatistik eine Konzentration der Volumina der externen FuEAufwendungen auf die Gruppe der Großunternehmen erkennen: Im Jahre 1999 entfielen auf Unternehmen mit über 500 Beschäftigten ca. $94 \%$ der gesamten externen FuE-Aufwendungen. Diese dominierende Rolle von Großunternehmen war auch für den gesamten betrachteten Zeitraum von 1979 bis 1999 feststellbar. Eine relativ größere Bedeutung der Gruppe der kleineren Unternehmen als Nachfrager von FuE-Dienstleistungen ließ sich nur in den 80er Jahren feststellen, was möglicherweise auf die Wirkung der Fördermaßnahme „Externe Vertragsforschung“ zurückgeführt werden kann. In diesem Zeitraum entfielen

443 Vgl. Fraunhofer-ISI/Prognos AG (1991), S. 236.

444 Vgl. Fraunhofer-ISI/Prognos AG (1991), S. 23. 
auf Unternehmen mit bis zu 499 Beschäftigten 18\%-34\% der gesamten externen FuE-Aufwendungen der deutschen Unternehmen.

Die Auswertung nach Wirtschaftszweigen (vgl. Tab. 5.2) zeigte, dass die chemische Industrie $(15,0 \%)$ und der Fahrzeugbau $(22,4 \%)$ die höchsten Anteile der externen FuE-Aufwendungen an den FuE-Gesamtaufwendungen im Jahre 1999 verzeichneten. Diese zwei Branchen prägten auch maßgeblich den für das gesamte Verarbeitende Gewerbe ermittelten Durchschnitt von $15,1 \%$. Bei der Analyse der Struktur der Auftraggeber zeigte sich, dass in allen betrachteten Branchen die größte Bedeutung der wirtschaftsinternen Auftragsvergabe zukam. Außerdem ergab sich für die chemische Industrie und den Fahrzeugbau - im Vergleich mit den Durchschnittwerten für das gesamte Verarbeitende Gewerbe - eine unterdurchschnittliche Bedeutung der Wissenschaft und eine überdurchschnittliche Bedeutung der ausländischen Akteure. Bei der Erläuterung der Ergebnisse der Sonderauswertung für Fahrzeugbau wurde zudem darauf hingewiesen, dass nicht nur die Kfz-Hersteller zur Auftragsvergabe neigen, sondern dass auch innnerhalb der Gruppe der Zulieferer Vertragsforschungsaktivitäten festgestellt werden konnten. ${ }^{445}$

Tabelle 5.2: Relativer Anteil und Struktur externer FuE-Aufwendungen der Unternehmen nach Wirtschaftszweigen (Anteile in \%)

\begin{tabular}{|c|c|c|c|c|}
\hline \multirow{2}{*}{ Wirtschaftszweig } & \multirow{2}{*}{$\begin{array}{l}\text { externe FuE / } \\
\text { FuE-Gesamt- } \\
\text { aufwendungen }\end{array}$} & \multicolumn{3}{|c|}{ Struktur der Auftragnehmer } \\
\hline & & Wirtschaft & Ausland & Wissenschaft \\
\hline Chemische Industrie & 15,06 & 67,44 & 26,08 & 6,47 \\
\hline Maschinenbau & 6,67 & 65,39 & 7,83 & 26,78 \\
\hline Elektrotechnik & 7,39 & 61,72 & 7,45 & 30,82 \\
\hline Fahrzeugbau & 22,38 & 73,92 & 19,10 & 6,97 \\
\hline \multicolumn{5}{|l|}{ Verarbeitendes } \\
\hline Gewerbe & 15,14 & 70,68 & 18,61 & 10,70 \\
\hline
\end{tabular}

Die Angaben beziehen sich auf das Jahr 1999.

Quelle: Auszug aus Tab. 3.6 in: Koschatzky/Reinhard/Grenzmann (2003), S. 57.

${ }^{445}$ Vgl. Koschatzky/Reinhard/Grenzmann (2003), S. 59. 
Für den Maschinenbau und die Elektrotechnik konnte ein Anteil der externen FuE-Aufwendungen von etwa $7 \%$ ermittelt werden. Bei der Analyse der relativen Bedeutung der Wissenschaft und des Auslandes in der Struktur der Auftragnehmer dieser Branchen ergab sich ein umgekehrtes Bild zur chemischen Industrie und zu dem Fahrzeugbau: eine überdurchschnittliche Bedeutung der Wissenschaft und eine unterdurchschnittliche Rolle des Auslandes. In der Elektrotechnik, dem Wirtschaftszweig mit dem höchsten Wertanteil von an wissenschaftliche Einrichtungen vergebenen FuE-Aufträgen, zeigte sich zudem, dass die Auftragsvergabe schwerpunktmäßig nicht an Hochschulen sondern an außeruniversitäre Forschungsinstitute erfolgte. In beiden Branchen wurde eine weniger stark ausgeprägte Dominanz der Großunternehmen festgestellt. Im Maschinenbau entfiel 1999 auf Betriebe mit bis zu 499 Beschäftigten ein 20\%iger Anteil an den gesamten externen FuE-Aufwendungen dieser Branche und im Jahr 1993 ein Anteil von über 37\%. ${ }^{446}$

Ein Blick auf die Forschungsintensitäten der Unternehmen (FuEAufwendungen im Verhältnis zum Umsatz) ließ erkennen, dass die Bedeutung der Vertragsforschung für forschungsintensivere Unternehmen größer war: Während für die FuE-Intensitätsklasse bis 3,5\% ein 5,3\%-iger Anteil der externen FuE-Aufwendungen an den FuE-Gesamtaufwendungen festgestellt werden konnte, lag der entsprechende Anteil in der Gruppe der Spitzentechnologieunternehmen (FuE-Intensität über $8,5 \%$ ) bei über $14 \%$ (vgl. Tab. 5.3). Der höchste Anteil (von 18.2\%) konnte in der Gruppe der Unternehmen mit mittlerer Forschungsintensität $(3,5 \%$ bis $8,5 \%)$ festgestellt werden. Für diese letzte Gruppe zeigte sich zudem bei der Untersuchung der Auftragnehmerstruktur eine unterdurchschnittliche Bedeutung der Wissenschaft und des Auslandes und eine verstärkte wirtschaftsinterne Verflechtung. Diese Unternehmen waren 1999 für über die Hälfte der FuE-Gesamtaufwendungen und über $62 \%$ der externen FuE-Aufwendungen der deutscher. Unternehmen verantwortlich. $^{447}$

Schließlich kam die Sonderauswertung der Wissenschaftsstatistik zu dem Ergebnis, dass Unternehmen, die Teil einer Unternehmensgruppe waren, einen höheren Anteil von externen FuE-Aufwendungen verzeichneten (16,9\%) als konzernunabhängige Unternehmen $(12,2 \%)$. Zwischen den Unternehmen im ausländischen und inländischen Eigentum zeigten sich nur geringe Unterschiede $(17,7 \%$ und $16,1 \%)$.

\footnotetext{
446 Vgl. Koschatzky/Reinhard/Grenzmann (2003), S. 58 sowie zu einzelnen Daten den Tabellen- und Abbildungsanhang, S. $204 \mathrm{ff}$.
}

447 Vgl. Koschatzky/Reinhard/Grenzmann (2003), S. 63. 
Tabelle 5.3: Relativer Anteil und Struktur externer FuE-Aufwendungen nach Forschungsintensitäten und Konzernabhängigkeit der Unternehmen (Anteile in \%)

\begin{tabular}{lcccc}
\hline \hline \multirow{2}{*}{ Merkmal } & \multicolumn{2}{c}{ Anteil } & \multicolumn{2}{c}{ Struktur der Auftragnehmer } \\
& externe FuE & Wirtschaft & Ausland & Wissenschaft \\
\hline Forschungsintensität & & & & \\
$\leq 3,5 \%$ & 5,27 & 54,12 & 31,34 & 24,50 \\
$3,5-8,5 \%$ & 18,18 & 76,52 & 15,50 & 8,02 \\
$\geq 8,5 \%$ & 14,16 & 61,88 & 24,20 & 13,92 \\
Konzernabhängigkeit der Unternehmen & & \\
konzernabhängig & 16,9 & 70,3 & 17,4 & 12,3 \\
konzernunabhängig & 12,2 & 67,0 & 22,9 & 10,1 \\
\hline \hline
\end{tabular}

Die Angaben beziehen sich auf das Jahr 1999.

Quelle: Auszüge aus Tab. 3.7 und Tab. 3.8 in: Koschatzky/Reinhard/Grenzmann (2003), S. $61 \mathrm{f}$.

\subsubsection{Ifo-Befragung im Verarbeitenden Gewerbe}

Das ifo Institut für Wirtschaftsforschung wurde im Rahmen der Schwerpunktstudien zur technologischen Leistungsfähigkeit Deutschlands 2001 vom Bundesministerium für Bildung und Forschung beauftragt, eine Studie zu FuEDienstleistungen zu erstellen. Im Rahmen des ifo-Innovationstests 2001 wurde hierfür eine Erhebung unter den Unternehmen des Verarbeitenden Gewerbes durchgeführt. Bei der Auswertung wurde ein Gewichtungsverfahren verwendet, so dass von einer Repräsentativität der Ergebnisse auszugehen ist. ${ }^{448}$

Von den befragten Unternehmen gaben $40 \%$ an, externe FuE-Dienstleistungen (FuE-Aufträge und sonstige externe technisch-wissenschaftliche Dienstleistungen zur Unterstützung der eigenen FuE-Tätigkeit) zu nutzen; 26,6\% der Unternehmen erteilte FuE-Aufträge an unternehmensexterne Partner. Es zeigte sich ein signifikanter Betriebsgrößeneffekt: Von den kleinen Unternehmen (unter 200 Beschäftigten) nutzten $29 \%$ externe FuE-Dienstleistungen; in der Gruppe der Unternehmen mit über 1000 Beschäftigen lag der Anteil bei über $75 \%$.

448 Vgl. Koschatzky/Reinhard/Grenzmann (2003), S. 145. 
Tabelle 5.4: Motive der Unternehmen bei der Nutzung externer FuEDienstleistungen nach Auftragnehmertypen (Häufigkeit der Nennung in \%)

\begin{tabular}{lcc}
\hline \hline Motiv & $\begin{array}{c}\text { private } \\
\text { Unternehmen }\end{array}$ & $\begin{array}{c}\text { Forschungs- } \\
\text { einrichtungen }\end{array}$ \\
\hline Verkürzung der Innovationszeiten & 49,8 & 32,3 \\
Integration komplementären Know-hows & 48,1 & 46,7 \\
Zugang zu technischer Ausrüstung & 35,6 & 45,9 \\
Quantitative Kapazitätsausweiterung & 34,4 & 22,4 \\
Interne FuE nicht rentabel & 27,3 & 21,3 \\
Nutzung von Grundlagenwissen & 26,5 & 51,3 \\
Senkung der FuE-Kosten & 14,4 & 17,0 \\
\hline \hline
\end{tabular}

Anmerkung: Mehrfachnennungen möglich.

Quelle: Koschatzky/Reinhard/Grenzmann (2003), S. 147.

Bei der Untersuchung von Motiven der Auftragsvergabe zeigte sich ein ähnliches Bild wie in der in Abschnitt 5.2.1 erläuterten Studie. Integration komplementären Know-hows und Zugang zu technischer Ausrüstung stellten - neben dem Motiv der Verkürzung von Innovationszeiten - die am häufigsten genannten Ziele des Bezugs externer FuE-Dienstleistungen von privaten Unternehmen dar (vgl. Tab. 5.4). Das Motiv der Verkürzung der Innovationszeiten wurde zudem signifikant häufiger bei der Inanspruchnahme privater Unternehmen im Vergleich zu öffentlichen Forschungseinrichtungen genannt; die Autoren der Studie interpretierten dieses Ergebnis als ein Hinweis auf die primäre Rolle des Zeitfaktors bei produktnahen Entwicklungsvorhaben. Erneut zeigte sich die relativ nachrangige Bedeutung des Kostenfaktors: Nur $14 \%$ der Unternehmen gaben an, dass die Senkung der FuE-Kosten eines der Ziele bei der externen Wissensbeschaffung darstelle. Wiederholt stellte sich zudem heraus, dass auf unternehmensexterne Partner bei internen Bedarfsschwankungen zurückgegriffen wird: Über ein Drittel der befragten Unternehmen nannte die quantitative Erweiterung der internen Personalkapazität als ein Motiv der Nutzung externer Dienstleister. Dieses Ergebnis interpretierten die Autoren als einen Hinweis auf die Entkopplung der internen Kapazität von der kurzfristigen Geschäftsentwicklung: Im Falle guter Auftragslage kann die interne FuE-Personalkapazität durch externen Bezug von FuE-Dienstleistungen ergänzt werden, bei Auftragsrückgängen können die Kapazitäten schnell ange- 
passt werden. ${ }^{449}$ Eine zusätzliche Auswertung nach Betriebsgrößenklassen zeigte ferner, dass das Motiv der quantitativen Kapazitätserweiterung signifikant häufiger von größeren Unternehmen genannt wurde: Die Hälfte der Betriebe mit über 1000 Beschäftigten und 28\% der Betriebe mit bis zu 200 Beschäftigten maß diesem Aspekt große Bedeutung bei.

Der Befund, dass die Vertragsforschung bei Auslastungsschwankungen eine Rolle spielen könnte, verdeutlicht die Bedeutung der in Abschnitt 3.3.1 erläuterten Problematik hoher Anpassungskosten der FuE-Tätigkeit. Es wurde darauf hingewiesen, dass sich Unternehmen möglicherweise darum bemühen, das FuE-Budget über mehrere Perioden zu glätten und nicht an die Schwankungen des Kapitalflusses zu koppeln. Nur dadurch kann der Diffusion des personengebunden Wissens zu den Wettbewerbern entgegengewirkt werden. Die Untersuchung der Motive der Auftragsvergabe zeigt, dass die Vertragsforschung ein Instrument darstellen kann, welches den Unternehmen hilft, bei positiver, jedoch in der Erwartung nicht dauerhafter Nachfrageentwicklung, dem gestiegenen FuE-Bedarf Rechnung zu tragen, ohne dass interne FuEKapazitäten aufgestockt - und bei antizipiertem Nachfragerückgang wieder abgebaut werden müssten. Die Vertragsforschung ermöglicht es folglich, die Schwankungen im FuE-Personalbestand - mit der Konsequenz des Wissensabflusses beim Personalabbau - zu vermeiden.

Neben Vorteilen der externen Wissensbeschaffung wurden in der Befragung auch Fragen nach den Hemmnisfaktoren gestellt. Am häufigsten wurde dabei der hohe Preis externer FuE-Dienstleistungen privater Unternehmen genannt $(70,9 \%)$. Dieses Hemmnis war insbesondere in der Gruppe der kleineren Unternehmen (bis zu 200 Beschäftigten) relevant (83,2\% der Nennungen.) Es wurde auch auf einige Gefahren der Vertragsforschung verwiesen: 60,8\% der Unternehmen nannten die Gefahr des Know-how-Abflusses, der Ungewissheit über die Leistungsqualität ( $57,6 \%$ der Nennungen), und allgemein die hohen Transaktionskosten externer Koordinierung (54,3\%). Der Gefahr des Know-how-Abflusses maßen insbesondere große Unternehmen (mit über 1000 Beschäftigten) eine große Bedeutung bei ( $82,2 \%$ der Nennungen) ${ }^{450}$

Die Erhebung ermöglichte eine Einschätzung darüber, welcher Anteil der Unternehmen FuE-Dienstleistungen durchführt. ${ }^{451}$ Es ergab sich ein Anteil von

449 Vgl. Koschatzky/Reinhard/Grenzmann (2003), S. 156.

$450 \mathrm{Zu}$ Motiven, Hemmnissen und allgemeinen Daten über die industriellen Nachfrager von FuE-Dienstleistungen vgl. Koschatzky/Reinhard/Grenzmann (2003), Kap. 5.

$451 \mathrm{Zu}$ nachfolgenden Daten vgl. Koschatzky/Reinhard/Grenzmann (2003), Abschnitt 4.7. 
$5,5 \%$, wobei ein signifikant positiver Betriebsgrößeneffekt verzeichnet werden konnte: Während nur 3,1\% der kleinsten Unternehmen (mit bis zu 50 Beschäftigten) FuE-Aufträge durchführten, lag der entsprechende Anteil in der Gruppe der größten Unternehmen bei $27,7 \%$.

Bei der Schätzung der durch Vertragsforschungsdienstleistungen erzielten Umsätze der privaten Unternehmen ergab sich ein Wert von rund 3,6 Mrd. EUR. Dabei stammten $82,3 \%$ der Einnahmen von inländischen Auftraggebern und $17,7 \%$ aus dem Ausland. Fast $40 \%$ der Erlöse resultierten aus Aufträgen der Kunden und nur 0,4\% aus Aufträgen der Lieferanten. Knapp 18\% der Einnahmen gingen auf konzerninterne Verflechtungen zurück.

Unter den Motiven der Durchführung von FuE-Dienstleistungen wurde am häufigsten die Möglichkeit der Gewinnung von zusätzlichem Know-how und die Nutzung von Synergieeffekten genannt (vgl. Tab. 5.5). Erneut zeigte sich die Bedeutung der Vertragsforschung bei Auslastungsschwankungen: 72,7\% der Unternehmen gaben an, das die Annahme externer FuE-Aufträge eine bessere Auslastung interner FuE-Kapazitäten gewährleistet. ${ }^{452}$ Somit liefern die Ergebnisse dieser Studie einen Hinweis auf die Gültigkeit der Hypothese 4 auch in der Gruppe der Vertragsforschungsanbieter.

Tabelle 5.5: Motive der Unternehmen bei der Durchführung von FuEDienstleistungen für externe Auftraggeber (Häufigkeit der Nennung in \%)

\begin{tabular}{lc}
\hline \hline Motiv & Anteil \\
\hline Zusätzliche Möglichkeiten der Gewinnung von Know-how & 88,7 \\
Nutzung von Synergieeffekten & 76,5 \\
Bessere Auslastung der internen Kapazitäten & 72,7 \\
Betätigung auf einem eigenständigen Geschäftsfeld & 59,2 \\
Nutzung von Größenvorteilen & 24,0 \\
Sonstige Motive & 22,9 \\
\hline \hline
\end{tabular}

Anmerkung: Mehrfachnennungen möglich.

Quelle: Koschatzky/Reinhard/Grenzmann (2003), S. 137.

452 Vgl. Koschatzky/Reinhard/Grenzmann (2003), S. 139. 


\subsubsection{Vertragsforschungsanbieter aus den neuen Bun- desländern}

Im Zuge des Transformationsprozesses der ostdeutschen Wirtschaft entstand eine Vielzahl von sogenannten "externen Industrieforschungseinrichtungen“. Es handelt sich hierbei um Wirtschaftsakteure, die als Ausgründungen von Hochschulen, der ehemaligen Akademie der Wissenschaften und der Kombinate entstanden sind. Die Zahl dieser Einrichtungen, die zum großen Teil dem Wirtschaftszweig „Forschung und Entwicklung für Unternehmen“ zugerechnet werden, wird in der einschlägigen Literatur auf 240 geschätzt. ${ }^{453}$ Am Ende der 90er Jahre hat das Bundesministerium für Wirtschaft und Technologie eine Studie zu den Rahmenbedingungen und Erfolgsfaktoren der Vertragsforschungsaktivitäten dieser Einrichtungen in Auftrag gegeben.

Eine schriftliche Befragung der Unternehmen, die der Gruppe der externen Industrieforschungseinrichtungen zugerechnet werden konnten, wurde 1998 durchgeführt. Von den 92 angeschriebenen Unternehmen sandten 56 verwertbare Fragebögen zurück. Diese relativ kleine Stichprobe ermöglichte - angesichts der ebenfalls relativ kleinen Grundgesamtheit - eine Analyse der spezialisierten Gruppe ostdeutscher Vertragsforschungsanbieter.

Die Analyse der erhobenen Daten zeigte, dass es sich bei den befragten Vertragsforschungsanbietern um relativ kleine Unternehmen handelte, die im Durchschnitt knapp 37 Mitarbeiter beschäftigten und einen Umsatz von 4,75 Mio. DM pro Jahr erzielten. Über $47 \%$ der erzielten Gesamteinnahmen stammten von der Vertragsforschung, weitere $28 \%$ aus der öffentlichen Förderung und $12 \%$ aus der industriellen Produktion. Hinsichtlich des Kriteriums des Anwendungsbezugs ließ sich der Gegenstand der Mehrheit der Vertragsforschungsprojekte $(50,5 \%)$ der Kategorie „Entwicklung“ zuordnen; der Anteil der Grundlagenforschung lag bei 5,5\%. Wie bei der Evaluierung der Fördermaßnahme „Externe Vertragsforschung“ zeigte sich folglich die große Anwendungsnähe der in Auftrag gegebenen FuE-Projekte. Zu den Gebieten, auf welchen die ostdeutschen Vertragsforschungsanbieter FuE-Dienstleistungen anboten, gehörten vorwiegend Umweltschutz und Umwelttechnik, Maschinen- und Gerätebau, Elektrotechnik, Elektronik, Mikroelektronik, Optik, Produktions/Verfahrenstechnik sowie Werkstoffentwicklung. ${ }^{454}$

453 Vgl. Rüdiger (2000), S. 212 f. sowie die dort zitierten Quellen.

454 Vgl. Rüdiger (2000), S. $227 \mathrm{ff}$. 
Zu den Auftraggebern der befragten Unternehmen gehörten vorwiegend private Unternehmen (zu 63\%), welche vor allem der Gruppe der Klein- und Mittelbetriebe (bis zu 250 Beschäftigte) zugeordnet werden konnten. Weniger als die Hälfte der privatwirtschaftlichen Nachfrager der FuE-Dienstleistungen kamen aus den alten Bundesländern, etwa $40 \%$ aus den neuen Bundesländern und knapp $12 \%$ aus dem Ausland. Die Untersuchung der Branchenstruktur der industriellen Kunden der befragten Unternehmen zeigte, dass über ein Viertel des Auftragvolumens von Unternehmen des Maschinenbaus stammte. Der Autor der Studie verwies in diesem Zusammenhang auf den hohen Anteil der Maschinenbaubranche an der FuE in den neuen Bundesländern. ${ }^{455}$

\subsubsection{Zwischenfazit}

Trotz der Unterschiede in der Schwerpunktlegung einzelner Studien und im speziellen Untersuchungsgegenstand wiederholen sich in den jeweiligen Untersuchungen einige Befunde, die nachfolgend festgehalten werden sollen.

Die Auswertung der Wissenschaftsstatistik sowie die Studie des ifo-Instituts kommen zu dem Ergebnis, dass die Nachfrageseite des FuE-Marktes in Deutschland insbesondere von der Tätigkeit der Großunternehmen geprägt wird. In der Evaluierung der Fördermaßnahme „Externe Vertragsforschung" sowie in der Auswertung der Wissenschaftsstatistik zeigt sich ferner, dass das Instrument der Vertragsforschung insbesondere von den forschenden - und forschungsintensiven - Unternehmen zur Ergänzung bzw. Verstärkung eigener FuE-Kompetenz herangezogen wird. Unter den Motiven der Auftragsvergabe spielen Zugang zu Know-how und apparativen Einrichtungen unternehmensexterner Partner sowie die Überwindung von Engpasssituationen eine herausragende Rolle. Dem Kostenfaktor kommt in der Einschätzung der Unternehmen nur eine untergeordnete Bedeutung zu. Allerdings weist die ifo-Studie darauf hin, dass Kosten- und Rentabilitätsüberlegungen implizit in den anderen genannten Motiven enthalten sind. ${ }^{456}$

Ferner stellen die Studie über die ostdeutschen Vertragsforschungsanbieter und die Evaluationen der Fördermaßnahme fest, dass von der Anwendungsnähe derjenigen Vorhaben auszugehen ist, die den Gegenstand von Vertragsforschungsbeziehungen bilden. Allerdings wurde in beiden Studien die Gruppe der Klein- und Mittelunternehmen betrachtet, einer Betriebsgrößenklasse, der

$455 \mathrm{Vgl}$. Rüdiger (2000), S. 238.

$456 \mathrm{Vgl}$. Koschatzky/Reinhard/Grenzmann (2003), S. 147 f. 
im Allgemeinen eine stärkere Anwendungsorientierung in der Forschungs- und Entwicklungstätigkeit zugestanden wird. Inwieweit der Befund der erheblichen Anwendungsnähe der entgeltlich erbrachten FuE-Dienstleistungen auch in der Gruppe der Großunternehmen festgestellt werden kann, lässt sich anhand der vorliegenden Daten nicht abschätzen.

Schließlich kann darauf hingewiesen werden, dass die meisten Studien von einer künftig steigenden Bedeutung externer FuE-Aufwendungen ausgehen. Bereits in der ältesten Studie zum FuE-Dienstleistungsmarkt in Deutschland von $1972^{457}$ wurde festgestellt, dass die Rolle der Vertragsforschung im Rahmen der gesamten FuE-Politik der Unternehmen weit über den damals statistisch ermittelten Anteil von 3-4\% hinausging und weiter an Bedeutung gewinnen dürfte. So wurde die Schlussfolgerung formuliert: „[D]ie Fallstudien erbrachten den Beweis, dass fortschrittliche Großfirmen sich ernsthafte Gedanken darüber machen, wie das Instrument der Vertragsforschung optimal in die gesamte FuE-Politik eines Unternehmens eingebaut werden kann. Der Weg der Zukunft dürfte daher wegführen von der heute noch üblichen, gelegentlichen Nutzbarmachung externer FuE-Ressourcen als Ausnahme zur Überbrückung von Engpässen hin zur systematischen Verwendung der Vertragsforschung als Mittel der rationalen Gestaltung und Organisation der eigenen FuE-Arbeit. “458

In der neuesten Untersuchung von 2003 ging man ebenfalls von einem weiteren Bedeutungszugewinn der externen FuE aus. ${ }^{459}$ So ergab die im Rahmen der ifo-Studie durchgeführte Erhebung, dass die befragten Unternehmen für 2001 einen Anstieg der externen FuE-Ausgaben gegenüber 2000 von knapp $6 \%$ planten. Dieses 6\%-ige Wachstum lag deutlich über dem geplanten Anstieg der gesamten FuE-Ausgaben von 4\%. Zudem ergab die Umfrage, dass während gut $28 \%$ der Unternehmen ihre externen FuE-Ausgaben im Jahre 2002 zu erhöhen planten, nur $17 \%$ die Ausgaben für den externen Bezug von FuE-Leistungen senken wollten. ${ }^{460}$ Die neuesten Daten der Wissenschaftsstatistik zur Entwicklung der externen FuE-Aufwendungen, wie sie im einleitenden Kapitel der Arbeit dargelegt wurden, bestätigen diese mit den unternehmerischen Angaben begründete positive Einschätzung der Wachstumsperspektiven des FuE-Marktes.

457 Vgl. Röthlingshöfer (1972).

458 Vgl. Röthlingshöfer (1972), S. 38.

459 Vgl. Koschatzky/Reinhard/Grenzmann (2003), S. 73 sowie S. 112.

460 Vgl. Koschatzky/Reinhard/Grenzmann (2003), S. 73. 


\subsection{Modellschätzungen I (MIP 1993)}

Nachdem im vorigen Abschnitt die vorliegende empirische Evidenz zum FuEDienstleistungsmarkt in Deutschland dargelegt wurde, erfolgt in diesem Abschnitt eine erste Schätzung der Determinanten des Angebots von bzw. der Nachfrage nach extern erbrachten FuE-Leistungen (Abschnitte 5.3.3 und 5.3.4). Die Datengrundlage dieser beiden Studien bildet zunächst die erste Erhebungswelle des Mannheimer Innovationspanels. Eine Schätzung anhand von Daten der letzten - für externe Nutzer zugänglichen - Erhebung aus dem Jahre 2003 wird in Abschnitt 5.4 vorgenommen.

\subsubsection{Anmerkungen zur Stichprobe}

In den nachfolgenden Analysen werden nur innovierende Unternehmen betrachtet, da lediglich für diese Unternehmen im Rahmen des MIP FuErelevante Daten erhoben werden. Als Innovatoren werden dabei diejenigen Unternehmen bezeichnet, die innerhalb der letzten drei Jahre Produkt- oder Prozessinnovationen durchgeführt bzw. zum Zeitpunkt der Erhebung die Entwicklung und/oder Markteinführung von Produkt- oder Prozessinnovationen in den nächsten drei Jahren geplant haben. Die Beschränkung auf Innovatoren führt zur Reduzierung der Stichprobengröße um 542 auf 2317 Unternehmen. Diese um Nichtinnovatoren bereinigte Stichprobe wird bei der Analyse der Verbreitung alternativer Koordinationsformen für FuE-Leistungen in Abschnitt 5.3.2 verwendet.

In den Regressionen kann nur auf die Beobachtungen zurückgegriffen werden, bei denen keine fehlenden Werte auftreten. Beim gegebenen komplexen Charakter der erhobenen Daten stehen nicht zu jeder Frage vollständige Angaben zur Verfügung. In den Schätzungen kann in der Folge auf reduzierte Stichproben im Umfang von 1379 Unternehmen (Abschnitt 5.3.3) bzw. 1389 Unternehmen (Abschnitt 5.3.4) zurückgegriffen werden. Die Zusammensetzung der um Nichtinnovatoren bereinigten Stichprobe des MIP 1993 sowie der in Schätzungen verwendeten Stichproben nach Wirtschaftszweigen, Betriebsgrößenklassen sowie neuen und alten Bundesländern befindet sich in Tab. 5.6.

Wie der Tab. 5.6 entnommen werden kann, sind bei der bivariaten Untersuchung der Zusammensetzung der jeweiligen Stichproben nur geringfügige Unterschiede feststellbar - und dies trotz der starken Reduzierung der Anzahl der Beobachtungen in den Datensätzen, die bei den Schätzungen verwendet 


\begin{tabular}{|c|c|c|c|}
\hline & $\begin{array}{c}\text { MIP } 1993 \\
(5.3 .2)\end{array}$ & $\begin{array}{l}\text { Stichpr. } 1 \\
(5.3 .3)\end{array}$ & $\begin{array}{l}\text { Stichpr. } 2 \\
(5.3 .4)\end{array}$ \\
\hline \multicolumn{4}{|l|}{ Wirtschaftszweige (nach WZ 1993) } \\
\hline 1) Bergbau (10-14) & 0,69 & 0,51 & 0,50 \\
\hline 2) Ernährung/Tabak (15-16) & 3,93 & 3,34 & 3,60 \\
\hline 3) Textil-/Bekleidung-/Ledergewerbe (17-19) & 4,06 & 4,64 & 4,54 \\
\hline \multicolumn{4}{|l|}{ 4) Holz-/Papier-/Verlags-/Druckgewerbe } \\
\hline$(20-22)$ & 4,45 & 3,99 & 3,38 \\
\hline 5) Chemische Industrie (23-24) & 7,29 & 8,19 & 8,78 \\
\hline 6) Gummi- und Kunststoffe (25) & 6,39 & 7,47 & 7,20 \\
\hline 7) Glasgewerbe, Keramik (26) & 3,24 & 3,41 & 3,67 \\
\hline \multicolumn{4}{|l|}{ 8) Herstellung von Metallen } \\
\hline und Metallerzeugnissen (27-28) & 12,65 & 12,76 & 13,25 \\
\hline 9) Maschinenbau (29) & 19,59 & 22,55 & 22,68 \\
\hline \multicolumn{4}{|l|}{ 10) EDV, Elektro-, und Nachrichtentechnik } \\
\hline$(30-32)$ & 8,46 & 9,64 & 8,71 \\
\hline 11) MMSR, Optik (33) & 6,82 & 7,61 & 8,06 \\
\hline 12) Fahrzeugbau (34-35) & 5,44 & 6,67 & 6,19 \\
\hline 13) Herstellung von MSMSS (36) & 3,37 & 3,70 & 3,74 \\
\hline \multicolumn{4}{|l|}{$\begin{array}{l}\text { 14) Recycling, Elektrizitäts- und Wasser- } \\
\text { versorgung, Abwasser und Abfall- }\end{array}$} \\
\hline beseitigung $(37,40-41,90)$ & 3,32 & 1,60 & 1,66 \\
\hline 15) Baugewerbe (45) & 3,71 & 3,48 & 3,46 \\
\hline \multicolumn{4}{|l|}{ 16) Datenverarbeitung und Datenbanken, FuE, } \\
\hline Dienstleistungen für Unternehmen (72-74) & 6,60 & 0,44 & 0,58 \\
\hline \multicolumn{4}{|l|}{ Betriebsgrößenklassen } \\
\hline 5-49 Beschäftigte & 29,82 & 26,25 & 26,49 \\
\hline 50-249 Beschäftigte & 32,28 & 31,83 & 30,96 \\
\hline über 250 Beschäftigte & 37,89 & 41,91 & 42,55 \\
\hline \multicolumn{4}{|l|}{ Bundesländer } \\
\hline alte Bundesländer & 67,37 & 68,60 & 69,26 \\
\hline neue Bundesländer & 32,63 & 31,40 & 30,74 \\
\hline \multicolumn{4}{|l|}{ Vertragsforschungsaktivitäten } \\
\hline Durchführung von FuE-Leistungen & 21,71 & 20,16 & \\
\hline Vergabe von FuE-Aufträgen & 32,17 & & 33,48 \\
\hline Anzahl der Beobachtungen & 2317 & 1379 & 1389 \\
\hline
\end{tabular}

Quelle: MIP 1993, eigene Auswertung. 
werden. Ein Blick auf die Branchenstruktur verdeutlicht, dass forschungsintensive Wirtschaftszweige wie die chemische Industrie, der Maschinenbau, die Messtechnik und Optik oder der Fahrzeugbau in den Schätzungsstichproben (estimation samples) etwas stärker vertreten sind als diejenigen Branchen, bei denen geringere FuE-Aufwendungen verzeichnet werden (wie das Holz-, Papier-, Verlags- und Druckgewerbe, die Elektrizitäts- und Wasserversorgung, die Abwasser und Abfallbeseitigung). Möglicherweise fällt es den forschungsintensiven Unternehmen leichter, spezielle innovationsrelevante Fragen zu beantworten wie beispielweise nach den Hemmnissen der Innovationstätigkeit oder nach der Wirksamkeit von Mechanismen zum Schutz unternehmensinterner Wissensvorsprünge. Besonders gewichtig ist jedoch nur die geringe $\mathrm{Be}-$ obachtungszahl in der Dienstleistergruppe: Dem über 6\%-igen Anteil in dem um Nichtinnovatoren bereinigten Datensatz des Mannheimer Innovationspanels steht ein unter 1\%-iger Anteil in beiden in den Schätzungen verwendeten Stichproben gegenüber.

Bei Betrachtung der Betriebsgrößenklassen und Regionen (neue bzw. alte Bundesländer) zeigt sich ferner, dass es in den bei der Schätzung verwendeten Stichproben zu einer leichten Verschiebung zugunsten der größeren Wirtschaftseinheiten und Unternehmen aus Westdeutschland kommt. Insgesamt ergeben sich jedoch keine gewichtigen Unterschiede bei den Anteilen der FuE-Aufträge durchführenden und erteilenden Unternehmen.

\subsubsection{Koordinationsformen für FuE-Leistungen}

In diesem Abschnitt wird der Frage nachgegangen, inwieweit unterschiedliche Koordinationsformen für die Beschaffung und Weitergabe neuen technologischen Wissens in der Subpopulation der innovierenden Unternehmen verbreitet sind. Hierzu stehen ausschließlich im MIP 1993 Angaben zur Nutzung der verschiedenen Kanäle des Technologietransfers zur Verfügung. Die Unternehmen wurden gefragt, welche von acht vorgegebenen Formen des Erwerbs bzw. der Weitergabe neuen technologischen Wissens im vergangenen Jahr genutzt wurden. ${ }^{461}$ Aus dieser Liste sind diejenigen ausgesucht worden, die in Übereinstimmung mit dem Systematisierungsansatz der Abb. 2.1 (S. 41) -

$461 \mathrm{Zu}$ den aufgelisteten Formen des Transfers neuen Wissens gehörten: 1) Lizenznahme bzw. -vergabe, 2) Vertragsforschung, 3) Beratungsdienstleistungen, 4) Kauf bzw. Verkauf eines anderen Unternehmens, 5) Gründung oder Beitritt zu einem joint venture, 6) Kauf bzw. Verkauf von Ausrüstungsgegenständen, 7) Erfahrungstausch und 8) Mobilität qualifizierten Personals. 
Tabelle 5.7: Verbreitung der Formen des Erwerbs neuen technischen Wissens

Form des Erwerbs

Anteil

der Unternehmen

Lizenznahme; Nutzungsrechte auf Erfindungen oder

Schutzrechte anderer

24,8

Erwerb der Forschungsergebnisse beauftragter

externer Unternehmen

32,2

Erfahrungstausch mit anderen Unternehmen

82,2

Gründung oder Beitritt zu einem joint venture

11,7

Kauf eines anderen Unternehmens/Geschäftsbereichs

15,7

Anmerkung: Mehrfachnennungen möglich.

Quelle: MIP 1993, eigene Auswertung.

den hybriden und externen Koordinationsformen für FuE zugeordnet werden können.

Wie der Tab. 5.7 entnommen werden kann, sind die über Eigenforschung hinausgehenden Koordinationsformen relativ stark verbreitet. Der Anteil der innovierenden Unternehmen, die bei der Beschaffung neuen technologischen Wissens auf eine durch Vertrag oder Absprache geregelte Zusammenarbeit mit anderen Wirtschaftsakteuren zurückgreifen, variiert je nach Art der Koordinationsform zwischen $12 \%$ und $82 \%$. Dabei zeigt sich eine eindeutige Präferenz für informale Formen der Zusammenarbeit. Über $82 \%$ der Unternehmen geben an, sich am Erfahrungstausch mit anderen Unternehmen zu beteiligen. Der Anteil der Unternehmen, die die institutionalisierte Kooperationsform eines joint ventures wählen, liegt dagegen nur bei $12 \%$. Für die externen Koordinationsformen der Beschaffung technologischen Wissens gilt: Während weniger als $25 \%$ der Unternehmen rechtlich geschützte Nutzungsrechte an Erfindungen erwerben, beauftragen über $32 \%$ der Unternehmen andere Wirtschaftseinheiten mit der Aufgabe, bestimmte Forschungsarbeiten durchzuführen und das gewonnene Wissen rückzutransferieren. Somit ist bei Betrachtung dieser Ergebnisse von einer stärkeren Verbreitung des Instruments der Vertragsforschung im Vergleich zur institutionalisierten Kooperationsform eines joint ventures auszugehen. 
Tab. 5.8 zeigt die Verbreitung der Formen der Weitergabe neuen technologischen Wissens. Der Vergleich mit Werten der Tab. 5.7 führt zu dem Ergebnis, dass die Gruppe der Forschungsdienstleister kleiner als die Gruppe der auftragserteilenden Firmen ausfällt: Während 32\% der innovierenden Unternehmen FuE-Aufträge vergibt, liegt der Anteil der FuE-Aufträge durchführenden Unternehmen an allen innovierenden Unternehmen bei knapp $22 \%$.

Tabelle 5.8: Verbreitung der Formen der Weitergabe neuen technischen Wissens

Form der Weitergabe

Anteil

der Unternehmen

Lizenzvergabe; Nutzungsrechte an eigenen Erfindungen

23,0

Durchführung der Forschungsaufträge für andere

Unternehmen

21,7

Erfahrungstausch mit anderen Unternehmen

69,4

Gründung oder Beitritt zu einem joint venture

11,3

Verkauf eines Teils des Unternehmens

4,7

Anmerkung: Mehrfachnennungen möglich.

Quelle: MIP 1993, eigene Auswertung.

Der anhand von Daten der ersten Welle des Mannheimer Innovationspanels ermittelte Anteil der FuE-Dienstleister unter allen Innovatoren von $22 \%$ unterscheidet sich damit erheblich von dem in der ifo-Studie berichteten Anteil von 5,5\%. Die Ursache kann dabei in der unterschiedlichen Zusammensetzung der Stichproben der beiden Erhebungen gesucht werden: Während in der ifoStudie nur Unternehmen des Verarbeitenden Gewerbes untersucht wurden, sind in dem MIP-Datensatz zusätzlich folgende Branchen repräsentiert: Bergbau, Baugewerbe, Elektrizitäts- und Wasserversorgung, Abwasser- und Abfallbeseitigung sowie wissensintensive Dienstleistungen ${ }^{462}$. Zudem wird in der

462 Als "wissensintensive Branchen des Dienstleistungssektors“ bzw. "wissensintensive Dienstleistungen" werden nachfolgend folgende Wirtschaftszweige bezeichnet: a) in der Abgrenzung der Klassifikation der Wirtschaftszweige aus dem Jahre 1993 (WZ 1993): Datenverarbeitung und Datenbanken (72), Forschung und Entwicklung (73), Erbringung von Dienstleistungen überwiegend für Unternehmen (74); b) in der Abgrenzung der Klassifikation der Wirtschaftszweige aus dem Jahre 2003 (Abschnitt 5.4): Datenverarbeitung und Datenban- 
vorliegenden Arbeit nur die Population der innovierenden Unternehmen untersucht.

Ferner fällt beim Vergleich der Werte der Tab. 5.7 mit denen der Tab. 5.8 auf, dass $82 \%$ der Unternehmen angeben, im Rahmen informeller Kooperationsbeziehungen Erfahrungswerte Dritter zu übernehmen, während nur $69 \%$ der Unternehmen angeben, eigene Erfahrungswerte an Dritte weiterzugeben. Dies wird erklärbar, wenn man die im Antwortverhalten zum Ausdruck kommende Diskrepanz als einen Hinweis auf die in der Williamsonschen Transaktionskostenökonomik stark betonte Tendenz zum opportunistischen Verhalten deutet. Zwar ist es das Ziel einer informellen Kooperationsbeziehung, Erfahrungswerte auszutauschen, aber möglicherweise versuchen die Wirtschaftsakteure im Rahmen einer solchen Kooperation Wissen Dritter zu internalisieren, ohne dabei durch Weitergabe eigenen innovationsrelevanten Wissens in Gegenleistung zu treten.

Innovatoren, die angaben, das Instrument der Vertragsforschung zu nutzen, wurden zudem gebeten, die geografische Herkunft der Auftragnehmer bzw. -geber zu nennen (Mehrfachnennungen waren möglich). Hierbei zeigte sich eine eindeutige Präferenz der deutschen Unternehmen für inländische Akteure: 91\% der mit der Durchführung einer FuE-Leistung beauftragten Wirtschaftsakteure kamen aus Deutschland und nur 17,8\% aus anderen Ländern der Europäischen Gemeinschaften (EG). Nur vereinzelt wurden Unternehmen aus anderen Ländern genannt (europäische nicht-EG-Länder, USA, Japan). Zu den Auftraggebern der deutschen FuE-Dienstleister gehörten ebenfalls vorwiegend inländische Akteure (82\%), gefolgt von Partnern aus EG-Ländern (25\%), den Vereinigten Staaten (13\%) und europäischen nicht EG-Ländern (12\%).

\subsubsection{Determinanten der Durchführung von FuE- Aufträgen}

In diesem Abschnitt wird zunächst der Frage nach den Bestimmungsfaktoren der Durchführung von FuE-Leistungen nachgegangen. Es sollen insbesondere die in der theoretischen Analyse formulierten Hypothesen einer ökonometrischen Prüfung unterzogen werden. Zudem gilt es jedoch auch einige weitere in der Literatur formulierte Hypothesen explizit zu berücksichtigen.

ken (72), Forschung und Entwicklung (73), Erbringung von wirtschaftlichen Dienstleistungen (74). 


\subsubsection{Spezifikation von Determinanten}

Die abhängige Variable der nachfolgenden Schätzung basiert auf der im Fragebogen des MIP 1993 gestellten Frage, ob die Firmen im vergangenen Jahr für andere Unternehmen FuE-Aufträge durchgeführt haben. Sie nimmt bei einer positiven (negativen) Antwort den Wert 1 (0) ein.

Die in der Schätzung verwendeten unabhängigen Variablen sind in Tab. 5.9 zusammengestellt. Sie sind aus Gründen der Übersichtlichkeit in sechs Gruppen zusammengefasst und sollen nachfolgend erläutert und in Beziehung zu den Hypothesen gestellt werden.

In Übereinstimmung mit den Hypothesen 3 und 9 ist $\mathrm{zu}$ erwarten, dass Unternehmen auf das Instrument der Vertragsforschung dann zurückgreifen, wenn sie Zugang zu spezifischen Ressourcen unternehmensexterner Partner erlangen wollen. Die FuE-Dienstleister sind folglich in der Gruppe derjenigen Unternehmen zu erwarten, die über bestimmte, im FuE-Bereich relevante Ressourcen und Kompetenzen verfügen.

Zur Erfassung spezifischer Ressourcen im FuE-Bereich werden drei Variablen berücksichtigt. Erstens wird mit einer Dummy-Variable (fuekon) die Eigenschaft der Unternehmen erfasst, die FuE-Aktivitäten kontinuierlich durchzuführen. Es kann erwartet werden, dass diejenigen Unternehmen intangible Kompetenzen aufbauen konnten und über spezielle Einrichtungen und Geräte verfügen, die sich auf kontinuierlicher Basis der Lösung bestimmter Problemstellungen widmen. Zweitens geht die Variable „Personalkostenintensität" (human, Personalaufwand im Verhältnis zum Umsatz) in die Schätzung ein, mit welcher - wie in der niederländischen Studie von den Hertog und Thurik - die Humanressourcen der Unternehmen erfasst werden. ${ }^{463}$ Vom positiven Einfluss dieser Variable auf die Wahrscheinlichkeit der Durchführung von FuE-Aufträgen ist insbesondere im Sinne der Hypothese 9 auszugehen. Zur Beschreibung der finanziellen Ressourcen wird die Variable finanz herangezogen, die auf der Angabe basiert, inwieweit aus der Sicht der jeweiligen Unternehmung fehlendes Eigenkapital ein Innovationshemmnis darstellt (gemessen auf einer Ordinalskala mit fünf Kategorien: keine Bedeutung bis sehr große Bedeutung). Es wird erwartet, dass diejenigen Unternehmen, die Finanzierungsbeschränkungen unterliegen, am FuE-Markt auf der Anbieterseite

463 Vgl. den Hertog/Thurik (1993), S. 285. 
Tabelle 5.9: Spezifikation der Determinanten der Durchführung von FuELeistungen

\begin{tabular}{|c|c|c|c|}
\hline Variable & Erläuterung & MW & SD \\
\hline & Unternehmenseigenschaften & & \\
\hline fuekon & kontinuierliche FuE (Dummy) & 0,65 & 0,48 \\
\hline human & Humankapitalintensität (Personalkosten/Umsatz) & 0,35 & 0,16 \\
\hline finanz & Fehlendes Eigenkapital ist ein Innovationshemmnis $\left({ }^{*}\right)$ & 3,09 & 1,45 \\
\hline bgk1 & Betriebsgrößenklasse: bis 50 Beschäftigte (Dummy) & 0,26 & 0,44 \\
\hline bgk3 & Betriebsgrößenklasse: über 250 Beschäftigte (Dummy) & 0,42 & 0,49 \\
\hline \multirow[t]{2}{*}{ ugrup } & Unternehmen ist Teil einer Unternehmensgruppe & & \\
\hline & (Dummy) & n.a. & n.a. \\
\hline risiko & Hohes Innovationsrisiko ist ein Innovationshemmnis $\left({ }^{*}\right)$ & 3,01 & 1,14 \\
\hline
\end{tabular}

Wirksamkeit der Schutzmechanismen

Faktoranalytische Komprimierung von 6 Mechanismen

zum Schutz des Wissens- bzw. Technikvorsprungs

gesetz Patente, Gebrauchsmuster

$0,02 \quad 0,76$

strateg Geheimhaltung, Komplexität, zeitlicher Vorsprung

langfristige Bindung qualifizierten Personals

$0,00 \quad 0,72$

Erfahrung

\begin{tabular}{llll} 
koop & Kooperationstätigkeit im Innovationsbereich (Dummy) & 0,39 & 0,49 \\
\hline Bedeutung unternehmensexterner Wissensquellen
\end{tabular}

Faktoranalytische Komprimierung von Informationsquellen

iwiss private und öffentliche Forschungseinrichtungen,

Patensschriften Universitäten, Fachhochschulen,

$\begin{array}{lrl}\text { Technologietransferstellen } & 0,01 & 0,88\end{array}$

$\begin{array}{lll}\text { izulief Zulieferer } & -0,01 & 0,70\end{array}$

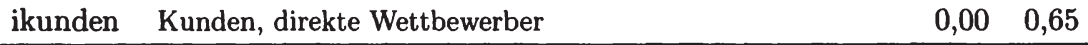

Marktbedingungen

wetint erwartete Wettbewerbsintensität in den nächsten

\begin{tabular}{llrr} 
& 3 Jahren $\left({ }^{*}\right)$ & 4,27 & 0,90 \\
\hline & Sonstige Variablen & & \\
ost & Unternehmen aus neuen Bundesländern (Dummy) & 0,31 & 0,46 \\
\hline \hline
\end{tabular}

MW: Mittelwert; SD: Standardabweichung; $\left({ }^{*}\right)$ Ordinalskalierte Variablen mit fünf Kategorien; n.a.: nicht anwendbar aufgrund der reduzierten Stichprobengröße. Quelle: MIP 1993, eigene Auswertung. 
auftreten. Durchführung von vergüteten FuE-Leistungen für Dritte ermöglicht diesen Unternehmen den Zugang zu zusätzlichen finanziellen Mitteln. ${ }^{464}$

Gemäß der Hypothese 6 ist ferner zu erwarten, dass mit steigender Unternehmensgröße die Koordinationsprobleme innerhalb einer Unternehmung zunehmen, was die Unternehmen dazu veranlasst, Teile der Forschungsaktivitäten an unternehmensexterne Partner zu vergeben. Die FuE-Dienstleister sind folglich verstärkt in der Gruppe der kleineren Unternehmen zu suchen, die weniger bürokratisch organisiert sind und Vorteile aufgrund von schwächer ausgeprägten Koordinationsproblemen genießen. Zur Prüfung dieser Hypothese werden zwei Dummy-Variablen berücksichtigt, die den Effekt der Zugehörigkeit zur Gruppe der kleinen Unternehmen (weniger als 50 Beschäftigte, Betriebsgrößenklasse 1, bgk1) und Großunternehmen (über 250 Beschäftigte, bgk3) erfassen. Die Referenzkategorie bildet die Gruppe der mittleren Unternehmen (50 bis 249 Beschäftigte).

Bei der Diskussion der Hartschen Modellierung des Property-Rights-Ansatzes ist ferner die Hypothese formuliert worden, dass Vertragsforschungsbeziehungen in der Folge einer Ausgliederung von FuE-Gesellschaften entstehen können (Hypothese 8). Zur Prüfung dieser Hypothese wird eine Dummy-Variable herangezogen (ugrup), die den Wert von 1 für rechtlich selbstständige Unternehmen einnimmt, die Teil einer Unternehmensgruppe sind.

Die letzte Variable der Gruppe „Unternehmenseigenschaften“ (risiko) ermöglicht es zu untersuchen, ob sich ein hohes Innovationsrisiko negativ auf die Entscheidung für die Durchführung von FuE-Dienstleistungen auswirkt. Sie basiert auf den einzelwirtschaftlichen Einschätzungen der Unternehmen, inwieweit hohes Risiko ein Innovationshemmnis darstellt (gemessen auf einer 5-stufigen Ordinalskala: keine Bedeutung bis sehr große Bedeutung).

In der transaktionskostenorientierten Analyse wurden die Gefahren der Vertragsforschung herausgestellt, die aufgrund der Tendenz zum opportunistischen Verhalten der Wirtschaftsakteure zustande kommen. Aus der Sicht der Forschungsdienstleister besteht die größte Gefahr im potenziellen Versuch des Auftraggebers, sich auch das innovationsrelevante Wissen des FuEDienstleisters anzueignen, welches keinen Bestandteil des FuE-Vertrages darstellt. Zum Schutz eigener Wissensvorsprünge vor einer ungewollten Diffusion sollten folglich die FuE-Dienstleister bemüht sein, Instrumente zu entwickeln, die es ihnen ermöglichen, den unkontrollierten Wissensabfluss zu verhindern. Anknüpfend an die Typologie von Levin et al. (1987) wurde im Fragebogen

464 Vgl. Pisano (1991), S. 240. 
der ersten Welle des Mannheimer Innovationspanels die Frage nach der Effektivität von sechs verschiedenen Instrumenten zum Schutz des Wissens- und Technikvorsprungs gestellt. Diese einzelwirtschaftlichen, subjektiven Angaben - faktoranalytisch zu zwei Variablen verdichtet - werden nachfolgend als eine Proxy für unternehmensspezifische Aneignungsbedingungen verwendet.

Die Ergebnisse der Faktoranalyse ${ }^{465}$ - nach der Varimax-Rotation - finden sich in Tab. 5.10. Hervorgehoben sind die für die Interpretation charakteristischen Faktorladungen. Der erste Faktor repräsentiert die Wirksamkeit der gesetzlichen, der zweite die der strategischen Maßnahmen zum Schutz innovationsrelevanten Wissens. Gemäß der Hypothese 1 wird erwartet, dass diejenigen Unternehmen, die die Wirksamkeit der Instrumente zum Schutz des Wissensvorsprungs höher einschätzen, eine größere Neigung aufweisen, FuEDienstleistungen anzubieten, d.h. ein positiver Einfluss der Variablen gesetz und strateg.

Tabelle 5.10: Ergebnisse der Faktoranalyse der Schutzmaßnahmen

\begin{tabular}{lcc}
\hline \hline Schutzmechanismen & $\begin{array}{c}\text { Faktor 1 } \\
\text { (gesetz) }\end{array}$ & $\begin{array}{c}\text { Faktor } 2 \\
\text { (strateg) }\end{array}$ \\
\hline Patente & $\mathbf{0 , 6 6}$ & 0,12 \\
Gebrauchsmuster, Copyright & $\mathbf{0 , 6 5}$ & 0,15 \\
Geheimhaltung & 0,33 & $\mathbf{0 , 4 4}$ \\
Komplexität der Produktgestaltung & 0,10 & $\mathbf{0 , 5 2}$ \\
Zeitlicher Vorsprung & 0,29 & $\mathbf{0 , 5 6}$ \\
Langfristige Bindung qualifizierten Personals & 0,06 & $\mathbf{0 , 4 4}$ \\
\hline \hline
\end{tabular}

Rotierte Faktorladungen (Varimax-Rotation); Eigenwerte: 1,60; 0,48. Quelle: MIP 1993, eigene Auswertung.

Eine weitere Möglichkeit, der Gefahr eines opportunistischen Verhaltens der Transaktionspartner entgegenzuwirken, besteht darin, eine Vertrauensbasis zwischen den Unternehmen aufzubauen. Im FuE-Bereich bietet sich hierfür die Möglichkeit, den Partner zunächst im Rahmen informeller Kooperationsbeziehungen kennenzulernen, bevor auf das Instrument der Vertragsforschung

465 Zum Verfahren der Faktor- bzw. Faktorenanalyse vgl. Lehrbücher der multivariaten Verfahren wie z.B. Johnson/Wichern (1999) oder Rinne (2000). In der vorliegen Studie wurde bei der Bestimmung der Faktorladungen die Hauptfaktorenmethode verwendet. 
zurückgegriffen wird. Zur Überprüfung der Frage, ob die in Kooperationsbeziehungen gesammelten Erfahrungen den von der Theorie erwarteten positiven Einfluss ausüben, wird die Dummy-Variable koop berücksichtigt, die auf der Angabe der Unternehmen darüber basiert, ob sie aktiv gemeinsame FuEProjekte mit anderen Unternehmen betreiben.

Die vierte Gruppe der Regressoren bilden Variablen, die aus der faktoranalytischen Komprimierung der einzelwirtschaftlichen Angaben zur Bedeutung von 9 verschiedenen unternehmensexternen Wissensquellen hervorgegangen sind. Die Ergebnisse der Faktoranalyse - nach der Varimax-Rotation - können der Tab. 5.11 entnommen werden. Die 3-Faktorenlösung ermöglicht folgende Interpretation: Der erste Faktor (iwiss) erfasst die Bedeutung der wissenschaftlichen Wissensquellen. Der zweite Faktor (izulief) repräsentiert die Bedeutung von Zulieferern von Vorprodukten und Ausrüstungsgegenständen. Der dritte Faktor (ikuwett) erfasst die Bedeutung von Kunden und Wettbewerbern.

Tabelle 5.11: Ergebnisse der Faktoranalyse der unternehmensexternen Wissensquellen

\begin{tabular}{lccc}
\hline \hline Wissensquellen & $\begin{array}{c}\text { Faktor 1 } \\
\text { (iwiss) }\end{array}$ & $\begin{array}{c}\text { Faktor 2 } \\
\text { (izulief) }\end{array}$ & $\begin{array}{c}\text { Faktor 3 } \\
\text { (ikunden) }\end{array}$ \\
\hline Zulieferer von Vorprodukten, Materialien, & & & \\
$\quad$ Komponenten & 0,06 & $\mathbf{0 , 6 0}$ & 0,12 \\
Zulieferer von Ausrüstungsgegenständen & 0,11 & $\mathbf{0 , 6 0}$ & 0,03 \\
Kunden & 0,14 & 0,10 & $\mathbf{0 , 5 4}$ \\
direkte Wettbewerber & 0,16 & 0,10 & $\mathbf{0 , 5 3}$ \\
industriefinanzierte Forschungseinrichtungen & $\mathbf{0 , 6 3}$ & 0,14 & 0,12 \\
Universitäten und Fachhochschulen & $\mathbf{0 , 7 3}$ & $-0,00$ & 0,09 \\
Großforschungseinrichtungen und sonstige & & & \\
technisch-wissenschaftliche Insitute & $\mathbf{0 , 7 9}$ & 0,05 & 0,06 \\
Technologietransferstellen & $\mathbf{0 , 6 6}$ & 0,15 & 0,09 \\
Patentschriften & $\mathbf{0 , 5 0}$ & $-0,05$ & 0,26 \\
\hline \hline
\end{tabular}

Rotierte Faktorladungen (Varimax-Rotation); Eigenwerte: 2,56; 0,75; 0,47. Quelle: MIP 1993, eigene Auswertung. 
Es kann vermutet werden, dass FuE-Dienstleistungen angeboten werden, wenn die Unternehmen durch die Zusammenarbeit mit Kunden bzw. Wettbewerbern Erkenntnisse über die Anwendung eigener Technologien erhalten wollen. Diesen Aspekt betont Pisano (1991) in seiner Studie der Organisationsstruktur des Pharma-Sektors. Er behauptet, dass Zugang zu nachgelagerten Kompetenzen (downstream capabilities) in Produktion, Durchführung klinischer Studien und Vermarktung fertiger Produkte eines der zentralen Motive für Biotechnologieunternehmen darstellte, die Vertragsforschungsdienstleistungen für Unternehmen der Pharma-Industrie anboten. Es wird ein positiver Einfluss der Variable ikunden erwartet.

Ferner kann argumentiert werden, dass Vertragsforschungsanbieter eine Schnittstelle zwischen den Forschungseinrichtungen und den industriellen Anwendern der wissenschaftlichen Erkenntnisse darstellen. Daher wird auch ein positiver Einfluss der Variable iwiss erwartet. Angesichts des häufig ermittelten negativen Einflusses einer großen Bedeutung von Zulieferern als externe Wissensquelle für Entscheidungen im FuE-Bereich, wird auf die Formulierung einer Hypothese über die Richtung des Einflusses der Variable izulief verzichtet.

In der Literatur wird häufig angeführt, dass der steigende Wettbewerbsdruck zur Veränderung der Organisationsstruktur privatwirtschaftlicher FuE führt. ${ }^{466}$ Diese Hypothese kann unter Zuhilfenahme einer Variable überprüft werden (wetint), die auf einer subjektiven Einschätzung der befragten Wirtschaftseinheiten bezüglich der erwarteten Entwicklung der Wettbewerbsintensität auf dem für eine Unternehmung relevanten Markt beruht. Die Entwicklung der Wettbewerbsintensität wurde auf einer Ordinalskala mit fünf Kategorien gemessen (erheblicher Rückgang bis starke Zunahme).

Schließlich soll wie in den Studien von Felder et al. (1996) und König und Licht (1995) die Variable ost berücksichtigt werden, mit welcher der besonderen Struktur und Wettbewerbssituation der Unternehmen aus den neuen Bundesländern Rechnung getragen wird.

466 Vgl. Radnor (1991), S. 113, Chung (1998), S. 41 sowie die dort zitierte Literatur. 


\subsubsection{Schätzergebnisse}

Aufgrund der dichotomen Ausprägung der abhängigen Variable wurde bei der Schätzung das Logit-Modell verwendet. ${ }^{467}$ Eine Kontrollschätzung unter Anwendung des Probit-Modells führte zu keinen abweichenden Ergebnissen im Hinblick auf Vorzeichen und Signifikanz der Variablen. Die Ergebnisse der Schätzungen der Logit-Modelle können der Tab. 5.12 entnommen werden.

Das erste angeführte Modell (1A) beinhaltet neben allen relevanten abhängigen Variablen Dummies für die in Tab. 5.6 aufgelisteten Branchen. Eine zweite Schätzung wurde unter Ausschluss der Branchendummies durchgeführt. Wegen des Unterschieds im Bayesschen Informationskriterium (BIC) von 79,6 ist dem Modell 1B (ohne Branchendummies) der Vorzug zu geben. ${ }^{468}$ Im dritten angeführten Modell (1C) ist zusätzlich die Variable ugrup berücksichtigt worden. Die Schätzung mit der Variable ugrup ist getrennt durchgeführt worden, da aufgrund von fehlenden Werten die Berücksichtigung dieser Variablen zu einer starken Reduzierung der Stichprobengröße um 384 Beobachtungen führt.

Der Likelihood-Ratio-Test lässt für alle Modelle die Hypothese verwerfen, dass alle Koeffizienten außer der Konstanten gleich Null sind. Der Wert für McFaddens-Pseudo- $r^{2}$ für die im Weiteren als Referenzmodell betrachtete Schätzung 1B liegt bei 0,18 , einem in der empirischen Innovationsforschung üblichen Wert. Zur weiteren Prüfung des Referenzmodells 1B wurden auch einflussreiche Fälle diagnostiziert unter Anwendung der von Pregibon (1981) vorgeschlagenen $\Delta \beta$-Statistik. ${ }^{469}$ Drei Kovariaten-Muster (mit je einer Beobachtung) waren besonders auffällig. In zwei Fällen handelte es sich dabei um kleine, nicht kontinuierlich forschende Innovatoren (unter 150 Beschäftigten), die zur Gruppe der Vertragsforschungsanbieter gehörten und in einem Fall um einen kleinen, forschungsintensiven Maschinenbauer, der keine FuE-Dienstleistungen

${ }^{467}$ Zum Logit-Modell vgl. insbesondere Long (1997), Hosmer/Lemeshow (1989). Zu Fragen der Interpretation der Logit-Modelle vgl. Liao (1994).

468 Richtlinien zur Anwendung des Bayesschen Informationskriteriums als einer ModelfitStatistik und einer Statistik, die zum Vergleich der Modelle herangezogen werden kann, wurden von Raftery (1996) vorgeschlagen. Im Allgemeinen gilt, dass dem Modell mit größerem negativen Wert des BIC der Vorzug zu geben ist. Folgende Intervalle wurden von Raftery (1996) bei der Beurteilung der Stärke der Evidenz für die Präferenz eines bestimmten Modells vorgeschlagen: 0 bis 2: schwacher Hinweis, 2 bis 6: positiver Hinweis, 6 bis 10: starker Hinweis, über 10: sehr starker Hinweis. Diesen Richtlinien wurde in dieser Arbeit gefolgt. Vgl. zu dieser Problematik auch Long/Freese (2001), S. 86 f.

469 Für eine lehrbuchartige Erläuterung der $\Delta \beta$-Statistik vgl. insbesondere Hosmer/Lemeshow (1989), Abschnitt 5.3 ab S. 149, auch Kohler/Kreuter (2001), S. $281 \mathrm{ff}$. 
Tabelle 5.12: Schätzergebnisse der Determinanten der Durchführung von FuEAufträgen

\begin{tabular}{|c|c|c|c|c|c|c|}
\hline \multirow{2}{*}{ Variable } & \multicolumn{2}{|c|}{ Modell 1A } & \multicolumn{2}{|c|}{ Modell 1B } & \multicolumn{2}{|c|}{ Modell 1C } \\
\hline & Koeff. & $\mathrm{t}$-Werte & Koeff. & t-Werte & Koeff. & t-Werte \\
\hline Konstante & $-5,83^{* * *}$ & $-8,33$ & $-5,03^{* * *}$ & $-9,85$ & $-4,48 * * *$ & $-7,69$ \\
\hline \multicolumn{7}{|c|}{ Unternehmenseigenschaften } \\
\hline fuekon & $1,35^{* * *}$ & 5,16 & $1,42^{* * *}$ & 5,59 & $1,28^{* * *}$ & 3,98 \\
\hline human & $1,26^{* *}$ & 2,37 & $1,47^{* *}$ & 2,89 & $1,02^{*}$ & 1,74 \\
\hline finanz & $0,10^{*}$ & 1,81 & $0,11^{*}$ & 1,86 & 0,05 & 0,78 \\
\hline bgk1 & 0,27 & 1,23 & 0,26 & 1,22 & $-0,04$ & $-0,10$ \\
\hline bgk3 & $-0,14$ & $-0,72$ & $-0,20$ & $-1,05$ & $-0,22$ & $-1,09$ \\
\hline ugrup & & & & & 0,12 & 0,67 \\
\hline risiko & 0,07 & 0,99 & 0,08 & 1,19 & 0,07 & 0,93 \\
\hline \multicolumn{7}{|c|}{ Wirksamkeit der Schutzmechanismen } \\
\hline gesetz & $-0,10$ & $-0,88$ & $-0,07$ & $-0,64$ & 0,11 & 0,85 \\
\hline strateg & $0,23^{*}$ & 1,80 & $0,22^{*}$ & 1,77 & 0,23 & 1,57 \\
\hline \multicolumn{7}{|l|}{ Erfahrung } \\
\hline koop & $1,25^{* * *}$ & 7,54 & $1,27^{* * *}$ & 7,65 & $1,21^{* * *}$ & 6,42 \\
\hline \multicolumn{7}{|c|}{ Bedeutung unternehmensexterner Wissensquellen } \\
\hline iwiss & $0,26^{* * *}$ & 2,72 & $0,24 * * *$ & 2,59 & $0,19^{*}$ & 1,79 \\
\hline izulief & $-0,17$ & $-1,54$ & $-0,18$ & $-1,62$ & $-0,15$ & $-1,18$ \\
\hline ikunden & 0,20 & 1,44 & $0,27^{*}$ & 1,95 & $0,30^{*}$ & 1,94 \\
\hline \multicolumn{7}{|c|}{ Marktbedingungen } \\
\hline wetint & $0,22^{* *}$ & 2,59 & $0,19^{* *}$ & 2,35 & $0,17^{*}$ & 1,74 \\
\hline ost & $-0,36^{*}$ & $-1,79$ & $-0,40^{* *}$ & $-1,98$ & $-0,13$ & $-0,54$ \\
\hline Branchendummies & \multicolumn{2}{|c|}{ ja } & \multicolumn{2}{|c|}{ nein } & \multicolumn{2}{|c|}{ nein } \\
\hline $\mathrm{n}$ & \multicolumn{2}{|c|}{1379} & \multicolumn{2}{|c|}{1379} & \multicolumn{2}{|c|}{995} \\
\hline Log-Likelihood & \multirow{2}{*}{\multicolumn{2}{|c|}{$-563,05$}} & \multirow{2}{*}{\multicolumn{2}{|c|}{$-570,24$}} & \multirow{2}{*}{\multicolumn{2}{|c|}{$-449,86$}} \\
\hline Likelihood-Ratio- & & & & & & \\
\hline$\chi^{2}$ & \multirow{2}{*}{\multicolumn{2}{|c|}{210,73}} & \multirow{2}{*}{\multicolumn{2}{|c|}{187,11}} & \multirow{2}{*}{\multicolumn{2}{|c|}{121,14}} \\
\hline McFaddens & & & & & & \\
\hline Pseudo- $r^{2}$ & \multicolumn{2}{|c|}{0,19} & \multicolumn{2}{|c|}{0,18} & \multicolumn{2}{|c|}{0,14} \\
\hline BIC & \multicolumn{2}{|c|}{$-8640,43$} & \multicolumn{2}{|c|}{$-8720,03$} & & \\
\hline
\end{tabular}

Huber/White-Schätzer der Varianz.

${ }^{* * *}$ Signifikant auf dem $1 \%$ Niveau; ${ }^{* *}$ signifikant auf dem $5 \%$ Niveau; *signifikant auf dem $10 \%$ Niveau.

Quelle: MIP 1993, eigene Auswertung. 
anbot, aber zur Gruppe der Nachfrager von FuE-Dienstleistungen gehörte. Eine Schätzung des Modells 1B unter Ausschluss dieser Beobachtungen veränderte jedoch die Vorzeichen bei den Koeffizienten nicht, lediglich ein Einfluss auf die Signifikanz von zwei Koeffizienten war feststellbar. Erstens erwies sich der Koeffizient bei der Variable $b g k 1$ als schwach signifikant $(p=0,098)$; der WaldTest ließ die Hypothese verwerfen $\left(\chi^{2}=6,02 ; 2\right.$ Freiheitsgrade, $\left.p<0,05\right)$, dass der Betriebsgrößeneffekt nicht feststellbar ist $(b g k 1=b g k 3=0)$. Zweitens verlor der Koeffizient bei der Variable strateg an Signifikanz $(p=0,131)$.

Im Lichte der in Tab. 5.12 dargestellten Schätzergebnisse (mit allen Beobachtungen) scheinen insbesondere die internen FuE-Kompetenzen der Unternehmen eine Voraussetzung für die Auftragsakquisition zu sein. Kontinuierlich forschende Unternehmen haben eine 4-fach höhere Chance $\left(e^{1,42}=4,14\right)$, FuEDienstleistungen für Dritte durchzuführen als Wirtschaftseinheiten, die sich der Lösung bestimmter Probleme im Rahmen interner FuE-Tätigkeit nur gelegentlich widmen bzw. keine internen FuE-Anstrengungen unternehmen. Auch Humankapitalintensität wirkt sich signifikant positiv auf die Wahrscheinlichkeit für die Durchführung von FuE-Leistungen aus. Kontinuierlich forschende humankapitalintensive Unternehmen verfügen offensichtlich über eigene Kompetenzen und materielle Ressourcen, auf die externe Partner bei der Vergabe von FuE-Verträgen zugreifen wollen. Diese Ergebnisse bestätigen somit die Befunde der empirischen Motivforschung, nach denen der Zugriff auf unternehmensexternes Know-how eines der zentralen Motive für die Auftragsvergabe bildet, sowie die Schätzergebnisse der Determinanten von Auftragsakquisition („Insourcing“ von FuE-Leistungen) in der Population der amerikanischen Unternehmen. ${ }^{470}$ In der letzten Studie kamen die Autoren ebenfalls zu der Schlussfolgerung, dass ,insourcing laboratories are more skill intensive than average ${ }^{\text {4 } 471}$.

Der Einfluss der Unternehmensgröße erweist sich in den dargestellten Modellen als nicht signifikant. Auch dieser Befund stimmt mit den Ergebnissen der Schätzungen in der Population der amerikanischen Unternehmen überein. ${ }^{472}$

470 In der auf den Daten zur FuE-Tätigkeit der US-amerikanischen Unternehmen basierenden Untersuchung von Adams und Marcu wurden die internen Kompetenzen der Forschungslaboratorien (industrial RED laboratories) mit einem Indikator abgebildet, der den Anteil des hochqualifizierten Personals unter den Mitarbeitern des jeweiligen FuE-Labors erfasste. In der Schätzung zeigte sich ein signifikant positiver Einfluss dieser Variable auf den Wertanteil der für Dritte durchgeführten FuE-Leistungen an dem gesamten FuE-Budget der Forschungslaboratorien, vgl. Adams/Marcu (2004).

471 Adams/Marcu (2004), S. 18.

472 Vgl. Adams/Marcu (2004), S. 25. 
Allerdings soll erneut darauf hingewiesen werden, dass bei der Schätzung des Modells 1B ohne einflussreiche Fälle der Koeffizient bei der Variable bgk1 signifikant wurde. Größenbedingte Vorteile der kleinen Unternehmen bei der Durchführung von FuE-Leistungen können folglich nicht ausgeschlossen werden.

Hohes Innovationsrisiko scheint für die Gruppe der FuE-Dienstleister kein bedeutendes Hemmnis zu sein. Gleichzeitig zeigt sich erwartungsgemäß, dass fehlendes Eigenkapital die Entscheidung für die Durchführung von FuEDienstleistungen begünstigt.

Der Einfluss von wirksamerem Schutz vor Wissensdiffusion durch formale Mittel (Patente und Gebrauchsmuster) erweist sich in der Schätzung als nicht signifikant, effektiverer Schutz eigener Wissensvorsprünge durch strategische Mittel (Geheimhaltung, Zeitvorsprung etc.) nur als schwach signifikant. Der Wald-Test lässt auf Unsignifikanz der Variablen schließen, die die Aneignungsbedingungen beschreiben $\left(H_{0}\right.$ : geset $z=$ strateg $=0, \chi^{2}=3,41 ; 2$ Freiheitsgrade; $p=0,18)$. Beide die Aneinungsbedingungen beschreibenden Variablen sind zudem im Modell 1C insignifikant. Berücksichtigt man darüber hinaus das Ergebnis der Schätzung ohne einflussreiche Fälle (fehlende Signifikanz der Variable strateg), dann kann man die Schlussfolgerung formulieren, dass Aneignungsbedingungen die Entscheidungen über die Durchführung von FuE-Leistungen nicht beeinflussen.

Einen stark positiven Einfluss übt die Variable koop aus. Kooperierende Innovatoren haben im Vergleich zu Unternehmen ohne Kooperationserfahrung eine 3,6-fach größere Chance $\left(e^{1,27}=3,56\right)$, als Vertragsforschungsanbieter zu fungieren.

Einen signifikant positiven Einfluss üben ferner erwartungsgemäß die Variablen iwiss aus, was auf eine höhere Wissenschaftsbindung der Vertragsforschungsanbieter im Vergleich zur Gruppe der sonstigen Innovatoren hinweist, sowie ikunden, was einen Hinweis auf Gültigkeit der Hypothese von Pisano (1991) liefert, wonach der Zugang zu Kompetenzen der Kunden einen der Gründe für das Anbieten von Vertragsforschungsdienstleistungen bildet. Der Einfluss der Variable izulief erweist sich dagegen als nicht signifikant.

Die erwartete Intensivierung des Wettbewerbs im Umfeld einer Unternehmung (Variable wetint) wirkt positiv auf die Neigung, FuE-Dienstleistungen anzubieten. Die Ergebnisse der Schätzungen bestätigen somit die in der Literatur häufig angeführte Hypothese, dass der verstärkte Wettbewerb zu Veränderung der Organisationsstruktur im FuE-Bereich führt. 
Unternehmen mit Sitz in den neuen Bundesländern haben eine 0,67-fach geringere Chance $\left(e^{-0,4}=0,67\right)$, zur Gruppe der Vertragsforschungsdienstleister zu gehören.

\subsubsection{Determinanten der Vergabe von FuE-Aufträgen}

Nachfolgend wird die Schätzung der Bestimmungsfaktoren der Auftragsvergabe vorgenommen. Erneut werden dabei neben den im theoretıschen Teil der Arbeit formulierten Hypothesen auch weitere in der Literatur vorzufindende Behauptungen hinsichtlich der Einflussfaktoren auf die Entscheidung für einen externen Bezug von FuE-Leistungen überprüft.

\subsubsection{Spezifikation von Determinanten der Auftragsvergabe}

Bei der Schätzung der Determinanten der Auftragsvergabe wird als abhängige Variable eine Dummy-Variable verwendet, die den Wert 1 für Unternehmen einnimmt, die im Jahr 1992 FuE-Aufträge an Dritte erteilt haben.

In Tab. 5.13 sind die in der Schätzung der Determinanten der Auftragsvergabe verwendeten Regressoren zusammengestellt. Sie sind erneut in sechs Gruppen zusammengefasst und sollen nachfolgend erläutert und in Beziehung zu den Hypothesen gestellt werden.

Die ersten beiden Regressoren der Gruppe „Unternehmenseigenschaften“ bilden zwei Dummy-Variablen: bgk1, die den Wert 1 annimmt, wenn die Unternehmen unter 50 Mitarbeiter beschäftigen und $b g k 3$, die den Wert 1 bei Unternehmen mit über 250 Beschäftigten annimmt. Gemäß der Hypothese 6 ist zu erwarten, dass große Unternehmen verstärkt auf das Instrument der Vertragsforschung zurückgreifen.

Drei weitere Variablen der Gruppe „Unternehmenseigenschaften“ (risiko, kosten und divers) werden herangezogen, um die in der Literatur häufig genannten Hypothesen über die Motive der Auftragsvergabe zu überprüfen. Hohes Innovationsrisiko stellt einen der Hemmnisfaktoren für unternehmensinterne FuE-Aktivität dar; es wir folglich oft postuliert, dass mit der Auftragsvergabe ein Versuch verbunden sei, die prozessinternen Risiken der FuE-Tätigkeit auf unternehmensexterne Partner zu übertragen. ${ }^{473}$ Daher wird erwartet, dass diejenigen Unternehmen, die dem Faktor hohes Innovationsrisiko eine große

${ }^{473} \mathrm{Vgl}$. die tabellarische Literatursynopse in Rüdiger (2000), S. 46. 
Tabelle 5.13: Spezifikation der Determinanten der Auftragsvergabe (MIP 1993)

\begin{tabular}{|c|c|c|c|}
\hline Variable & Erläuterung & MW & SD \\
\hline & Unternehmenseigenschaften & & \\
\hline bgk1 & Betriebsgrößenklasse: bis 50 Beschäftigte (Dummy) & 0,26 & 0,44 \\
\hline bgk3 & Betriebsgrößenklasse: über 250 Beschäftigte (Dummy) & 0,43 & 0,49 \\
\hline risiko & hohes Innovationsrisiko ist ein Innovationshemmnis $\left({ }^{*}\right)$ & 3,04 & 1,14 \\
\hline kosten & zu hohe Innovationskosten sind ein Innovationshemmnis $\left({ }^{*}\right)$ & 3,21 & 1,22 \\
\hline \multirow[t]{2}{*}{ divers } & Diversifizierung (Kehrwert des Herfindal-Index der & & \\
\hline & Produktgruppenumsätze; 4 umsatzstärkste Produkte) & 2,14 & 1,26 \\
\hline ugrup & Unternehmen ist Teil einer Unternehmensgruppe (Dummy) & n.a. & n.a. \\
\hline umver & Umsatzentwicklung in den vergangenen 3 Jahren $\left(^{*}\right)$ & 3,41 & 1,43 \\
\hline \multirow[t]{2}{*}{ expint } & Exportintensität (Exporte/Umsatz) & & \\
\hline & Absorptionsfähigkeit & & \\
\hline \multirow[t]{3}{*}{ fuekon } & kontinuierliche FuE (Dummy) & 0,65 & 0,48 \\
\hline & Bedeutung unternehmensexterner Wissensquellen & & \\
\hline & Faktoranalytische Komprimierung von Informationsquellen & & \\
\hline \multirow[t]{3}{*}{ iwiss } & private und öffentliche Forschungseinrichtungen, & & \\
\hline & Patensschriften, Universitäten, Fachhochschulen, & & \\
\hline & Technologietransferstellen & 0,03 & 0,88 \\
\hline izulief & Zulieferer & $-0,01$ & 0,69 \\
\hline \multirow[t]{3}{*}{ ikunden } & Kunden, direkte Wettbewerber & 0,00 & 0,65 \\
\hline & Wirksamkeit der Schutzmechanismen & & \\
\hline & $\begin{array}{l}\text { Faktoranalytische Komprimierung von } 6 \text { Mechanismen } \\
\text { zum Schutz des Wissens- bzw. Technikvorsprungs }\end{array}$ & & \\
\hline gesetz & Patente, Gebrauchsmuster & 0,02 & 0,75 \\
\hline \multirow[t]{3}{*}{ strateg } & Geheimhaltung, Komplexität, zeitlicher Vorsprung & & \\
\hline & langfristige Bindung qualifizierten Personals & 0,01 & 0,71 \\
\hline & Erfahrung & & \\
\hline \multirow[t]{2}{*}{ koop } & Kooperationstätigkeit im Innovationsbereich (Dummy) & 0,40 & 0,49 \\
\hline & Marktbedingungen & & \\
\hline \multirow[t]{2}{*}{ wetint } & erwartete Wettbewerbsintensität in den nächsten 3 Jahren $\left({ }^{*}\right)$ & 4,27 & 0,91 \\
\hline & Sonstige Variablen & & \\
\hline ost & Unternehmen aus neuen Bundesländern (Dummy) & 0,31 & 0,46 \\
\hline
\end{tabular}

MW: Mittelwert; SD: Standardabweichung; * Ordinalskalierte Variablen mit fünf Kategorien; n.a.: nicht anwendbar aufgrund der reduzierten Stichprobengröße. Quelle: Eigene Darstellung. 
Bedeutung beimessen, eine stärkere Neigung aufweisen, auf das Instrument der Vertragsforschung zurückzugreifen.

Zum anderen wird häufig die Hypothese formuliert, dass auf das Instrument der Vertragsforschung zurückgegriffen wird, wenn durch Auftragsvergabe eine Senkung der Kosten der FuE-Tätigkeit erwartet wird. ${ }^{474}$ Zur Überprüfung dieser Hypothese wird die Variable kosten berücksichtigt, die auf den einzelwirtschaftlichen Angaben darüber basiert, inwieweit zu hohe Innovationskosten ein Innovationshemmnis darstellen (gemessen auf einer Ordinalskala mit fünf Kategorien).

Es wird ferner postuliert, dass die zunehmende Komplexität und Überlappung von Technologien zur Veränderung der Organisationsstruktur im FuEBereich führt: Da einzelne Unternehmen unternehmensinterne Kompetenzen nicht auf jedem relevanten Gebiet aufbauen können, sind sie auf den Rückgriff auf das Know-how unternehmensexterner Partner angewiesen. ${ }^{475}$ Es kann vermutet werden, dass insbesondere stark diversifizierte Unternehmen mit breiter Produktpalette auf einen Zugriff auf externe Ressourcen und Kompetenzen angewiesen sind. ${ }^{476}$ Zur Erfassung des Diversifikationsgrades einer Unternehmung wird ein Indikator verwendet, der als Kehrwert des Herfindal-Index der vier umsatzstärksten Produktgruppen berechnet wurde. Die Variable divers nimmt den Wert 1 ein, wenn der gesamte Umsatz eines Unternehmens auf ein Produkt bzw. eine Produktgruppe fällt und steigt mit zunehmendem Diversifizierungsgrad eines Unternehmens. Erwartet wird ein positiver Einfluss dieser Variable.

Gemäß der Hypothese 4 kann Vertragsforschung als ein Instrument der Engpassüberwindung eingesetzt werden. Zur Überprüfung dieser Hypothese wird die Variable umver herangezogen, die auf den einzelwirtschaftlichen Einschätzungen der Umsatzentwicklung in den vergangenen drei Jahren basiert (gemessen auf einer 5-stufigen Ordinalskala: erheblicher Rückgang bis erhebliche Zunahme). Es wird erwartet, dass stark wachsende Unternehmen interne FuE-Kapazitäten nicht zeitgleich erweitern können und die Erforschung bestimmter Zusammenhänge zum Teil auf unternehmensexterne Partner verlagern müssen.

\footnotetext{
474 Vgl. Rüdiger (2000), S. 46.

475 Vgl. Hermes (1995), S. 1, Radnor (1991), S. 119, Koschatzky/Reinhard/Grenzmann (2003), S. 149, sowie ebenfalls Rüdiger (2000), S. 46.
}

476 Vgl. hierzu auch Beneito (2003), S. 707. 
Die Variable expint beschreibt die Exportintensität der Unternehmen (Exporte im Verhältnis zum Umsatz). Sie wird berücksichtigt, um für die Population der deutschen Unternehmen den Einfluss der Exporttätigkeit zu untersuchen; in den Schätzungen der Determinanten der Auftragsvergabe in der Population der finnischen Unternehmen hat sich ein hochsignifikant positiver Einfluss dieser Variable gezeigt. ${ }^{477}$

Für die Übernahme des extern erarbeiteten Wissens sind in Übereinstimmung mit der Hypothese 10 interne Absorptionsfähigkeiten eine Voraussetzung. Ein positiver Einfluss der internen FuE-Kompetenz wird auch gemäß der Hypothese 7 erwartet. Zur Erfassung der Absorptionsfähigkeit wird die Variable fuekon verwendet. Es wird davon ausgegangen, dass diejenigen Unternehmen externes Wissen internalisieren können, die über eigene Kompetenzen und Erfahrungen verfügen, die in kontinuierlicher Forschungstätigkeit aufgebaut werden konnten. ${ }^{478}$

Der Zugriff auf unternehmensexterne Ressourcen ist in den Hypothesen 3 und 9 als eines der zentralen Motive für die Auftragsvergabe herausgestellt worden. Zur Erfassung des Einflusses der Bedeutung des unternehmensextern vorhandenen Wissens wird erneut auf die Variablen zurückgegriffen, die aus der faktoranalytischen Komprimierung der einzelwirtschaftlichen Angaben zur Bedeutung von 9 verschiedenen Wissensquellen hervorgegangen sind (vgl. Tab. 5.11).

Gemäß der Hypothese 1 ist ferner zu erwarten, dass besserer Schutz vor unfreiwilliger Wissensdiffusion zum FuE-Dienstleister die Entscheidung für Auftragsvergabe begünstigen sollte. Zur Erfassung der Möglichkeiten des Schutzes innovationsrelevanter Wettbewerbsvorteile werden die aus der Faktoranalyse hervorgegangenen Variablen verwendet (gesetz und strateg, vgl. Tab. 5.10).

Wie in der Schätzung der Determinanten der Durchführung von FuELeistungen wird schließlich ein positiver Einfluss der Variablen koop und wetint erwartet.

\subsubsection{Schätzergebnisse}

Bei der Schätzung der Determinanten der Auftragsvergabe wurde erneut das Logit-Modell verwendet. Die Ergebnisse der Schätzungen der Logit-Modelle

477 Vgl. Leiponen (2002), S. 273.

478 Vgl. Cohen/Levinthal (1990), S. 138. 
sind in Tab. 5.14 aufgelistet. Das erste angeführte Modell (2A) beinhaltet erneut Branchendummies; eine zweite Schätzung ist unter Ausschluss der Branchendummies durchgeführt worden. Wegen des Unterschieds im BIC von 96,7 ist erneut dem Modell ohne Branchendummies der Vorzug zu geben. Wiederholt ist ebenfalls aufgrund der Problematik fehlender Werte eine getrennte Schätzung unter Berücksichtigung der Variable ugrup durchgeführt worden (Modell 2C). Ferner wurde das Modell 2B unter Ausschluss der nicht signifikanten Variablen (kosten, divers, gesetz und strateg) geschätzt. Die geschätzten Koeffizienten unterschieden sich jedoch dabei von den in Tab. 5.14 angeführten Ergebnissen der Schätzung des Modells 2B nur nach der zweiten Kommastelle und werden daher nicht gesondert aufgeführt. Eine Kontrollschätzung unter Anwendung des Probit-Modells führte ebenfalls zu keinen abweichenden Ergebnissen.

Eine Schätzung des Modells 2B unter Ausschluss von 4 Kovariaten-Mustern, die bei der Analyse der $\Delta \beta$-Statistik besonders auffällig waren, führte zu keinen Unterschieden in den Vorzeichen und Signifikanzen der Koeffizienten mit einer Ausnahme: Der Koeffizient bei der Variable strateg erwies sich als signifikant $(p=0,079)$. Die Hypothese über den fehlenden Einfluss der Appropriierungsbedingungen $($ strateg $=$ geset $z=0$ ) konnte jedoch auch in der Schätzung ohne einflussreiche Fälle nicht verworfen werden $\left(\chi^{2}=3,10\right.$; 2 Freiheitsgrade; $p=0,21)$.

Der McFaddens-Pseudo- $r^{2}$ von 0,26 (Modell 2B) ist höher als die entsprechende Statistik in der Schätzung der Determinanten der Durchführung von FuE-Aufträgen $(0,18)$ und auch höher als in der Probit-Schätzung der Determinanten der Auftragsvergabe in der Population der finnischen Unternehmen $(0,17) \cdot{ }^{479}$

In der Schätzung der Determinanten der Auftragsvergabe zeigt sich ein signifikanter Betriebsgrößeneffekt, ein Ergebnis, welches in Untersuchungen anhand von Daten zur FuE-Tätigkeit von US-amerikanischen und finnischen Unternehmen ebenfalls erzielt wurde. ${ }^{480} \mathrm{Im}$ Vergleich zur Referenzkategorie (mittlere Unternehmensgröße) haben kleine Unternehmen eine 0,6-fache geringere

479 Vgl. Leiponen (2002), S. 274.

$480 \mathrm{Vgl}$. Adams/Marcu (2004), Leiponen (2002). Lediglich in der niederländischen Studie von van Hertog und Thurik (1993) zeigte sich ein negativer Einfluss der Unternehmensgröße auf den Anteil der externen FuE (gemessen mit der Anzahl der extern beschäftigten FuEMitarbeiter an der Gesamtzahl der Beschäftigen des jeweiligen Unternehmens sowie an dem intern und extern mit der Durchführung von FuE-Leistungen beauftragten Personal), vgl. den Hertog/Thurik (1993), S. 280. 
Tabelle 5.14: Schätzergebnisse der Determinanten der Auftragsvergabe (MIP 1993)

\begin{tabular}{|c|c|c|c|c|c|c|}
\hline \multirow{2}{*}{ Variable } & \multicolumn{2}{|c|}{ Modell 2A } & \multicolumn{2}{|c|}{ Modell 2B } & \multicolumn{2}{|c|}{ Modell 2C } \\
\hline & Koeff. & t-Werte & Koeff. & $\mathrm{t}$-Werte & Koeff. & t-Werte \\
\hline Konstante & $-4,50^{* * *}$ & $-3,63$ & $-3,68^{* * *}$ & $-7,37$ & $-3,59^{* * *}$ & $-6,73$ \\
\hline \multicolumn{7}{|c|}{ Unternehmenseigenschaften } \\
\hline bgk1 & $-0,49 * *$ & $-2,35$ & $-0,49 * *$ & $-2,38$ & 0,37 & 0,83 \\
\hline bgk3 & $0,42^{* *}$ & 2,49 & $0,41^{* *}$ & 2,51 & $0,29 *$ & 1,71 \\
\hline risiko & $0,13^{*}$ & 1,80 & $0,12^{*}$ & 1,79 & 0,06 & 0,75 \\
\hline kosten & 0,03 & 0,39 & 0,02 & 0,28 & 0,07 & 0,91 \\
\hline ugrup & & & & & 0,18 & 1,15 \\
\hline divers & $-0,02$ & $-0,41$ & $-0,02$ & $-0,32$ & $-0,00$ & $-0,08$ \\
\hline umver & $0,13^{* *}$ & 2,40 & $0,11^{* *}$ & 2,18 & $0,10^{*}$ & 1,87 \\
\hline expint & $1,61^{* * *}$ & 4,92 & $1,59^{* * *}$ & 5,01 & $1,27^{* * *}$ & 3,74 \\
\hline \multicolumn{7}{|c|}{ Absorptionsfähigkeit } \\
\hline fuekon & $0,41^{* *}$ & 2,16 & $0,41^{* *}$ & 2,28 & $0,55^{* *}$ & 2,57 \\
\hline \multicolumn{7}{|c|}{ Bedeutung unternehmensexterner Wissensquellen } \\
\hline iwiss & $0,66^{* * *}$ & 7,94 & $0,65^{* * *}$ & 7,87 & $0,64^{* * *}$ & 6,70 \\
\hline izulief & $-0,22^{* *}$ & $-1,99$ & $-0,24^{* *}$ & $-2,37$ & $-0,21^{*}$ & $-1,82$ \\
\hline ikunden & $-0,07$ & $-0,52$ & $-0,05$ & $-0,41$ & $-0,03$ & $-0,21$ \\
\hline \multicolumn{7}{|c|}{ Wirksamkeit der Schutzmechanismen } \\
\hline gesetz & $-0,00$ & $-0,00$ & 0,00 & 0,00 & 0,00 & 0,03 \\
\hline strateg & 0,17 & 1,42 & 0,16 & 1,42 & 0,11 & 0,87 \\
\hline \multicolumn{7}{|l|}{ Erfahrung } \\
\hline koop & $1,38^{* * *}$ & 9,11 & $1,35^{* * *}$ & 9,23 & $1,28^{* * *}$ & 7,76 \\
\hline \multicolumn{7}{|l|}{ Marktbedingungen } \\
\hline wetint & $0,15^{*}$ & 1,90 & $0,14^{*}$ & 1,88 & $0,14^{*}$ & 1,65 \\
\hline \multicolumn{7}{|l|}{ Sonstige Variablen } \\
\hline ost & $0,32^{*}$ & 1,76 & $0,31^{*}$ & 1,72 & 0,10 & 0,50 \\
\hline Branchendummies & \multicolumn{2}{|c|}{ ja } & \multicolumn{2}{|c|}{ nein } & \multicolumn{2}{|c|}{ nein } \\
\hline $\mathrm{n}$ & \multicolumn{2}{|c|}{1389} & \multicolumn{2}{|c|}{1389} & \multicolumn{2}{|c|}{1006} \\
\hline Log-Likelihood & \multicolumn{2}{|c|}{$-653,08$} & \multicolumn{2}{|c|}{$-658,98$} & \multicolumn{2}{|c|}{$-527,47$} \\
\hline Likelihood-Ratio- $\chi^{2}$ & \multicolumn{2}{|c|}{329,04} & \multicolumn{2}{|c|}{316,22} & \multicolumn{2}{|c|}{225,16} \\
\hline \multicolumn{7}{|l|}{ McFaddens } \\
\hline Pseudo- $r^{2}$ & \multicolumn{2}{|c|}{0,26} & \multicolumn{2}{|c|}{0,26} & \multicolumn{2}{|c|}{0,22} \\
\hline $\mathrm{BIC}$ & \multicolumn{2}{|c|}{$-8513,55$} & \multicolumn{2}{|c|}{$-8610,30$} & & \\
\hline
\end{tabular}

Huber/White-Schätzer der Varianz. ${ }^{* * *}$ Signifikant auf dem $1 \%$ Niveau; ${ }^{* *}$ signifikant auf dem $5 \%$ Niveau; ${ }^{*}$ signifikant auf dem $10 \%$ Niveau.

Quelle: MIP 1993, eigene Auswertung. 
Chance $\left(e^{-0,49}=0,61\right)$, FuE-Verträge an unternehmensexterne Partner zu erteilen. Große Unternehmen (mit über 250 Beschäftigten) haben dagegen eine 1,5-fache größere Chance $\left(e^{0,41}=1,51\right)$, zur Gruppe der Auftraggeber zu gehören. Der Koeffizient bei der Variable bgk1 verliert an Signifikanz, wenn (in der reduzierten Stichprobe) für die Zugehörigkeit zur Unternehmensgruppe kontrolliert wird.

Von den Hemmnisfaktoren der Innovationstätigkeit spielt im Lichte der Schätzergebnisse nur die Risikoeinstellung eine Rolle. Naheliegend ist folglich, die Möglichkeit zur Externalisierung von Risiken der FuE-Tätigkeit als eines der relevanten Motive für die Auftragsvergabe zu betrachten. Der Einfluss der Diversifizierung erweist sich als nicht signifikant.

Eine positivere Umsatzentwicklung wirkt sich positiv auf die Entscheidung für Auftragsvergabe aus, ein ebenfalls in der US-amerikanischen Schätzung erzieltes Ergebnis. ${ }^{481}$ Können schnell wachsende Unternehmen ihre interne FuEKapazitäten nicht zeitgleich aufbauen, so greifen sie auf das Instrument der Vertragsforschung zurück.

Die Wahrscheinlichkeit für die Vergabe von FuE-Leistungen steigt - wie in der finnischen Studie von Leiponen (2002) - auch mit der Exportintensität der Unternehmen.

Das Vorhandensein von Absorptionsfähigkeiten, operationalisiert durch die Variable fuekon, scheint eine notwendige Voraussetzung für die Vergabe von FuEVerträgen an Dritte zu sein. Kontinuierlich forschende Unternehmen haben eine 1,5 -fache größere Chance $\left(e^{0,41}=1,51\right)$, externe FuE-Dienstleistungen nachzufragen.

Der Einfluss vom wirksameren Schutz vor Wissensdiffusion durch formale (Patente und Gebrauchsmuster) und informale Mittel (Geheimhaltung, zeitlicher Vorsprung etc.) zeigt sich in der Schätzung als nicht signifikant. Einen signifkant positiven Einfluss übt dagegen erwartungsgemäß die Variable iwiss aus. Aneignungsbedingungen scheinen folglich die Entscheidungen für Auftragsvergabe nicht zu beeinflussen. Zugleich verstehen es die Auftraggeber, extern zugängliche wissenschaftliche Wissensquellen für unternehmensinterne Zwecke zu verwerten.

481 Vgl. Adams/Marcu (2004), S. 25. In der niederländischen Studie von van Hertog und Thurik wirkte sich das Marktwachstum signifikant positiv auf die Intensität der externen FuE (Anteil der extern beschäftigten FuE-Mitarbeitern an der Gesamtzahl der Beschäftigten des jeweiligen Unternehmens) und insignifikant (positiv) auf den Anteil der externen FuE an der gesamten FuE-Leistung einer Unternehmung, vgl. den Hertog/Thurik (1993), S. 286. 
Interessanterweise stimmen die Ergebnisse der Schätzung in der Population der deutschen Unternehmen mit den Befunden der finnischen Studie von Leiponen (2002) über den Einfluss der technologischen Möglichkeiten auf Entscheidungen im Bereich der Vergabe von FuE-Verträgen vollständig überein trotz der Unterschiede in der Konstruktion von Indikatoren. Während in der vorliegenden Studie die auf Unternehmensebene erfassten Angaben zur Bedeutung von unternehmensexternen Wissensquellen faktoranalytisch zu drei Variablen verdichtet werden, gehen bei Leiponen die Angaben zur Bedeutung von Wettbewerbern, Kunden, Zulieferern und Universitäten als Branchendurchschnitte in die Schätzung ein. Trotzdem zeigt sich in der finnischen Probit-Schätzung ein gleiches Muster der Einflussgrößen: ein signifikant positiver Einfluss des Wissens von wissenschaftlicher Einrichtungen, ein signifikant negativer Einfluss des Wissens von Zulieferern und ein insignifikanter Einfluss des Wissens von Kunden und Wettbewerbern. ${ }^{482}$

Zudem wird bei der Schätzung der Determinanten der Auftragsvergabe ein ähnliches Ergebnis erzielt wie bei Schätzungen der allgemeinen Bestimmungsgründe der FuE-Tätigkeit. Bei letzteren wird auch häufig ein negativer $\mathrm{Zu}$ sammenhang zwischen der Bedeutung der Zulieferer als unternehmensexterner Wissensquelle und interner FuE-Tätigkeit ermittelt. Eine hohe Einschätzung der Zulieferer führt zu einer Senkung eigener FuE-Anstrengungen der Unternehmen und zur Reduzierung der Wahrscheinlichkeit der Vergabe von FuEVerträgen. Die Betrachtung beider Befunde lässt folgende Interpretation zu: Auch wenn Zulieferer als wichtige Wissensquelle eingeschätzt werden, wird das von ihnen erarbeitete Wissen nicht als immaterielles Produkt in Form von Ergebnissen ihrer Forschungstätigkeit zugekauft. Es kann vermutet werden, dass das innovationsrelevante Wissen der Zulieferer in der materiellen Form - als Bestandteil neuer Produkte bzw. Verfahren - erworben wird. Diese Einschätzung repräsentieren auch die Autoren der in Abschnitt 5.2.3 erläuterten Studie zum FuE-Dienstleistungsmarkt in Deutschland. Sie stellen fest: „In der Vergangenheit wurden Entwicklungsaufträge der Kunden an ihre Lieferanten separat bezahlt. Gegenwärtig gehen die OEM (produzierende Endkunden, Anmerkung der Autorin) in der Automobilindustrie dazu über, Entwicklungsaufträge nicht mehr direkt $\mathrm{zu}$ finanzieren. Sie möchten eine Vorfinanzierung vermeiden und stattdessen die Entwicklungsleistungen über die Preise der bezogenen Produkte bezahlen." 483

482 Vgl. Leiponen (2002), S. 274.

483 Koschatzky/Reinhard/Grenzman (2003), S. 155. 
Schließlich zeigt sich in der Schätzung der Determinanten der Auftragsvergabe eine positive Beziehung zwischen der Kooperationstätigkeit und der Neigung zur Auftragsvergabe sowie der positive Einfluss der antizipierten Entwicklung der Wettbewerbsintensität. Erneut können folglich Hinweise auf die Gültigkeit der Hypothese über den Einfluss der Wettbewerbsbedingungen auf die Organisationsstruktur im FuE-Bereich gefunden werden.

\subsection{Modellschätzungen II (MIP 2003)}

\subsubsection{Anmerkungen zur Stichprobe}

Der Datensatz des MIP 2003 unterscheidet sich von dem der Erhebung des Jahres 1993 insbesondere dadurch, dass mehrere Dienstleistungsbranchen wie beispielweise Handel, Transport oder Grundstücks- und Wohnungswesen berücksichtigt wurden. Um einen Vergleich der Schätzergebnisse für die Erhebungsjahre 1993 und 2003 zu ermöglichen, sind Unternehmen dieser Branchen aus dem Datensatz entfernt worden. Ferner beschränkt sich die Analyse erneut auf die Gruppe der innovierenden Unternehmen, d.h. Wirtschaftseinheiten, die in den vorangegangenen drei Jahren neue oder merklich verbesserte Produkte oder Prozesse eingeführt haben bzw. die Entwicklung und/oder die Markteinführung von neuen oder verbesserten Produkten oder Verfahren nicht abgeschlossen bzw. abgebrochen haben. Nach dem Ausschluss ausgewählter Dienstleistungsbranchen und Nichtinnovatoren reduziert sich die Beobachtungszahl auf 2169 Unternehmen. Diese Stichprobe wird bei der Betrachtung der Verbreitung des Instruments der Auftragsvergabe in Abhängigkeit von Strukturmerkmalen der Unternehmen in Abschnitt 5.4.2 zugrunde gelegt.

In den nachfolgenden Schätzungen der Determinanten der Auftragsvergabe wird eine reduzierte Stichprobe von 1150 Unternehmen zugrunde gelegt, die nach der Eliminierung der Beobachtungen mit fehlenden Werten zustande gekommen ist. Ihre Zusammensetzung kann der Tab. 5.15 entnommen werden.

Bei der bivariaten Betrachtung der Zusammensetzung beider Stichproben zeigen sich erneut - trotz der starken Reduzierung der Stichprobengröße von 2169 auf 1150 Unternehmen - nur geringfügige Abweichungen. Eine Analyse der Branchenstruktur lässt erkennen, dass folgende Branchen in der Schätzungsstichprobe verstärkt repräsentiert sind: chemische Industrie, Glasgewerbe/Keramik, EDV, Nachrichten-, Elektro-, Mess-, Medizintechnik, Optik und wissensintensive Dienstleistungen. Der relative Anteil dieser Branchen 
erhöht sich jedoch nur um insgesamt 3,69 Prozentpunkte. Es kommt zugleich zu einer leichten Verschiebung zugunsten der kleineren Unternehmen (unter 249 Beschäftigten). Auffällig ist jedoch, dass es zu einer Erhöhung des Anteils der Unternehmen mit Vertragsforschungsaktivitäten von $31,2 \%$ in der Stichprobe des MIP 2003 auf $40,0 \%$ in der reduzierten Stichprobe kommt. Es kann folglich festgehalten werden, dass die Stichprobe, die bei den Schätzungen der Determinanten der Auftragsvergabe verwendet wird, die Anteile der auftragserteilenden Unternehmen überschätzt.

Im Vergleich zu der in Abschnitt 5.3.4 - bei der Schätzung der Determinanten der Auftragsvergabe anhand des Datensatzes der ersten Welle des Mannheimer Innovationspanels - verwendeten Stichprobe (vgl. Tab. 5.6) ergeben sich folgende Unterschiede. Mehrere Branchen werden in der Stichprobe von MIP 2003 im Vergleich zur Stichprobe von MIP 1993 unterrepräsentiert. Dazu gehören neben Branchen mit geringeren Forschungsintensitäten (Textil-, Bekleidung- und Ledergewerbe, Herstellung von Möbel, Schmuck, Musikinstrumenten und Sportgeräten) auch diejenigen Branchen, bei denen bei bivariater Betrachtung in beiden Beobachtungsjahren hohe Anteile derjenigen Unternehmen festgestellt werden können, die FuE-Verträge an Dritte vergeben (chemische Industrie, Maschinenbau, Fahrzeugbau). Die Verschiebung in den relativen Anteilen ergibt sich daraus, dass Unternehmen der wissensintensiven Dienstleistungsbranchen (Datenverarbeitung und Datenbanken, FuE, Dienstleistungen für Unternehmen) verstärkt repräsentiert sind. Um zu überprüfen, inwieweit die Dienstleistungsunternehmen die Schätzergebnisse beeinflussen, sind weitere Schätzungen unter Ausschluss jener Wirtschaftseinheiten durchgeführt worden. Sie werden nach der Vorstellung der Ergebnisse der Referenzmodelle in Abschnitt 5.4.3.2 näher erläutert.

Unterschiede ergeben sich auch bei der Betrachtung der Zusammensetzung beider Stichproben nach Betriebsgrößenklassen. Sowohl in der um Nichtinnovatoren und einige Dienstleistungsbranchen bereinigten Stichprobe des Mannheimer Innovationspanels 2003 (zweite Spalte der Tab. 5.15) als auch in der Stichprobe, die bei den nachfolgenden Schätzungen zugrunde gelegt wird (dritte Spalte der Tab. 5.15), kommt es - im Vergleich zu den Stichproben der ersten Welle des Mannheimer Innovationspanels - zu einer Verschiebung der relativen Anteile zugunsten der kleinen und mittleren Unternehmen. Dies kann auch im Wesentlichen auf die starke Berücksichtigung der Unternehmen der wissensintensiven Branchen des Dienstleistungssektors zurückgeführt werden: $81 \%$ dieser Unternehmen beschäftigen weniger als 249 Mitarbeiter. Inwieweit die verstärkte Berücksichtigung der im Durchschnitt kleineren Unternehmen 
Tabelle 5.15: Zusammensetzung der Stichproben (MIP 2003)

\begin{tabular}{|c|c|c|}
\hline & $\begin{array}{c}\text { MIP } 2003 \\
\text { (Innovatoren) }\end{array}$ & $\begin{array}{l}\text { Stichprobe } 1 \\
\text { (Regression) } \\
\end{array}$ \\
\hline \multicolumn{3}{|l|}{ Wirtschaftszweige (nach WZ 2003) } \\
\hline 1) Bergbau (10-14) & 0,78 & 0,35 \\
\hline 2) Ernährung/Tabak (15-16) & 3,32 & 3,13 \\
\hline 3) Textil-/Bekleidung-/Ledergewerbe (17-19) & 3,09 & 2,87 \\
\hline 4) Holz-/Papier-/Verlags-/Druckgewerbe (20-22) & 4,01 & 3,65 \\
\hline 5) Chemische Industrie (23-24) & 5,81 & 6,00 \\
\hline 6) Gummi- und Kunststoffe (25) & 4,98 & 4,70 \\
\hline 7) Glasgewerbe, Keramik (26) & 2,95 & 3,13 \\
\hline \multicolumn{3}{|l|}{ 8) Herstellung von Metallen } \\
\hline und Metallerzeugnissen (27-28) & 9,08 & 8,43 \\
\hline 9) Maschinenbau (29) & 11,53 & 11,04 \\
\hline 10) EDV, Elektro-, und Nachrichtentechnik (30-32) & 7,28 & 7,91 \\
\hline 11) MMSR, Optik (33) & 8,02 & 8,43 \\
\hline 12) Fahrzeugbau (34-35) & 4,29 & 4,17 \\
\hline 13) Herstellung von MSMSS (36) & 2,81 & 2,61 \\
\hline \multicolumn{3}{|l|}{$\begin{array}{l}\text { 14) Recycling, Elektrizitäts- und Wasser- } \\
\text { versorgung, Abwasser und Abfall- }\end{array}$} \\
\hline beseitigung $(37,40-41,90)$ & 3,87 & 3,39 \\
\hline 15) Baugewerbe (45) & 1,75 & 1,48 \\
\hline \multicolumn{3}{|l|}{ 16) Datenverarbeitung und Datenbanken, FuE, } \\
\hline Dienstleistungen für Unternehmen (72-74) & 26,42 & 28,70 \\
\hline \multicolumn{3}{|l|}{ Betriebsgrößenklassen } \\
\hline 5-49 Beschäftigte & 43,38 & 44,87 \\
\hline 50-249 Beschäftigte & 28,63 & 30,96 \\
\hline über 250 Beschäftigte & 27,11 & 24,17 \\
\hline fehlende Angaben & 0,88 & n.a. \\
\hline \multicolumn{3}{|l|}{ Bundesländer } \\
\hline alte Bundesländer & 66,67 & 65,65 \\
\hline neue Bundesländer & 33,29 & 34,35 \\
\hline fehlende Angaben & 0,04 & n.a. \\
\hline Vergabe von FuE-Aufträgen & 31,17 & 40,00 \\
\hline Anzahl der Beobachtungen & 2169 & 1150 \\
\hline
\end{tabular}

n.a.: Nicht anwendbar aufgrund von Bereinigung der Stichprobe um Beobachtungen mit fehlenden Werten.

Quelle: MIP 2003, eigene Auswertung. 
der wissensintensiven Dienstleistungsbranchen Einfluss auf die Schätzergebnisse ausübt, kann folglich ebenfalls bei der Schätzung unter Ausschluss dieser Beobachtungen kontrolliert werden.

Es bleibt jedoch festzuhalten, dass die Unterschiede in der Zusammensetzung der Stichproben der Erhebungswellen 1993 und 2003 auf die Veränderung der Strukturmerkmale der innovierenden deutschen Unternehmen zurückgeführt werden können.

\subsubsection{Deskriptive Statistik}

Bevor in Abschnitt 5.4.3.2 die Schätzung der Determinanten der Auftragsvergabe vorgenommen wird, soll nachfolgend eine bivariate Analyse der Beteiligung an der Vertragsforschung in Abhängigkeit von zentralen Strukturmerkmalen der Unternehmen vorgenommen werden. Die Auswertung basiert auf der in der zweiten Spalte der Tab. 5.15 beschriebenen Stichprobe und bezieht sich auf Unternehmen, die die jeweils relevanten Informationen übermittelt haben.

\subsubsection{Auftragsvergabe und Unternehmensgröße}

Bei Betrachtung der Auswertung nach Betriebsgrößenklassen (vgl. Tab. 5.16) fällt zunächst auf, dass der Anteil der Unternehmen, die FuE-Aufträge an Dritte vergeben, stark mit der Unternehmensgröße steigt: Er liegt bei 30\% in der Gruppe der kleinen Unternehmen, bei $37 \%$ in der Gruppe der mittleren Unternehmen und bei knapp $58 \%$ in der Gruppe der Großunternehmen. Über die Hälfte der innovierenden Großunternehmen greift demnach in ihrer Innovationsstrategie auf das Instrument der Vertragsforschung zurück. Diese Ergebnisse bestätigen die Befunde der ifo-Studie über die Relevanz des Betriebsgrößeneffektes bei einer Betrachtung der Nutzung externer FuE-Dienstleistungen. 
Tabelle 5.16: Beteiligung an Vertragsforschung nach Betriebsgrößenklassen

\begin{tabular}{lc}
\hline \hline Betriebsgrößenklassen & Anteil der Unternehmen \\
\hline 5-49 Beschäftigte & 30,4 \\
$50-249$ Beschäftigte & 37,3 \\
über 250 Beschäftigte & 57,9 \\
\hline \hline
\end{tabular}

Quelle: MIP 2003, eigene Auswertung.

\subsubsection{Auftragsvergabe und Eigentumsverhăltnisse der Unterneh- men}

Bei der Erfassung der Unternehmensprofile wird im Fragebogen des MIP die Frage berücksichtigt, ob es sich bei der berichterstattenden Unternehmenseinheit um ein rechlich selbstständiges Einzelunternehmen oder einen rechtlich selbstständigen Teil einer Unternehmensgruppe handelt. Dies ermöglicht die Beantwortung der Frage, inwieweit unterschiedliche Innovationsstrategien in Abhängigkeit von Eigentumsverhältnissen beobachtet werden können. Die Auswertung zeigt, dass Unternehmen, die Teil einer Unternehmensgruppe sind, signifikant häufiger $\left(\chi^{2}=58,6 ; p<0,00\right)$ FuE-Dienstleistungen nachfragen (vgl. Tab. 5.17). Über die Hälfte dieser Unternehmen beteiligt sich an der Auftragsvergabe. Der entsprechende Anteil liegt in der Gruppe der Einzelunternehmen bei $32 \%$.

Tabelle 5.17: Beteiligung an Vertragsforschung nach Eigentumsverhältnissen

\begin{tabular}{lcccc}
\hline \multirow{2}{*}{ Eigentumsverhältnisse } & \multirow{2}{*}{ insgesamt } & \multicolumn{3}{c}{ Betriebsgrößenklasse } \\
& & bis 49 & $50-249$ & über 250 \\
\hline Einzelunternehmen & 31,9 & 29,1 & 31,1 & 50,5 \\
Teil einer Unternehmensgruppe & 50,3 & 37,1 & 43,9 & 60,2 \\
\hline \hline
\end{tabular}

Quelle: MIP 2003, eigene Auswertung.

Die zusätzliche Differenzierung nach Unternehmensgröße (erfasst durch die Anzahl der Mitarbeiter) zeigt, dass der positive Effekt der Zugehörigkeit zu 
einer Unternehmensgruppe auch unabhängig von der Betriebsgrößenklasse der Unternehmen gilt.

\subsubsection{Auftragsvergabe und Forschungsintensität der Unterneh- men}

Ein Blick auf die Forschungsintensitäten der einzelnen Unternehmen (vgl. Tab. 5.18) lässt erkennen, dass die Neigung zur Auftragsvergabe mit der Forschungsintensität der Unternehmen (gemessen als Anteil der FuE-Aufwendungen am Umsatz) steigt. Während über ein Drittel der Unternehmen mit einer Forschungsintensität von bis zu 3,5\% FuE-Aufträge erteilt, liegt der Anteil der Nachfrager von FuE-Dienstleistungen in der Gruppe der Unternehmen mit einer Forschungsintensität von über $8,5 \%$ bei über $60 \%$.

Tabelle 5.18: Beteiligung an Vertragsforschung nach Forschungsintensität der Unternehmen

\begin{tabular}{lcccc}
\hline \hline \multirow{2}{*}{ Forschungsintensität } & \multirow{2}{*}{ insgesamt } & \multicolumn{3}{c}{ Betriebsgrößenklasse } \\
& & bis 49 & $50-249$ & über 250 \\
\hline$\leq 3,5 \%$ & 34,3 & 19,8 & 32,7 & 54,0 \\
$3,5-8,5 \%$ & 50,6 & 34,7 & 41,5 & 84,2 \\
$\geq 8,5 \%$ & 60,8 & 53,7 & 76,5 & 79,4 \\
\hline \hline
\end{tabular}

Quelle: MIP 2003, eigene Auswertung.

Bei der Ausdifferenzierung nach Betriebsgrößenklassen zeigt sich zudem, dass der Anteil der auftragserteilenden Unternehmen sowohl mit der Unternehmensgröße als auch mit der Forschungsintensität der Unternehmen steigt. Während nur knapp $20 \%$ der kleinen Innovatoren (mit bis zu 50 Beschäftigten) mit einer Forschungsintensität von bis zu 3,5\% externe FuE-Dienstleistungen nachfragt, liegt der entsprechende Anteil in der Gruppe der Unternehmen mit über 250 Beschäftigten und einer Forschungsintensität von über $8,5 \%$ bei knapp $80 \%$. Der externe Bezug von FuE-Leistungen ist demnach besonders stark in der Population der großen und forschungsintensiven Unternehmen verbreitet. 


\subsubsection{Auftragsvergabe nach alten und neuen Bundesländern}

Die bivariate Betrachtung lässt kaum Unterschiede zwischen Unternehmen aus den neuen und alten Bundesländern erkennen (vgl. Tab. 5.19). Der $\chi^{2}$-Test lässt auf jeden Fall auf einen insignifikanten Unterschied $\left(\chi^{2}=0,00 ; p=0,97\right)$ zwischen ost- und westdeutschen Unternehmen schließen. Eine zusätzliche Ausdifferenzierung nach Betriebsgrößenklassen zeigt, dass sowohl für Unternehmen mit Sitz in den alten Bundesländern als auch für Betriebe aus den neuen Bundesländern ein positiver Betriebsgrößeneffekt feststellbar ist. Allerdings neigen kleine ostdeutsche Unternehmen häufiger zur Auftragsvergabe als kleine westdeutsche Betriebe. Ein umgekehrter Zusammenhang gilt für die großen Unternehmen (mit über 250 Beschäftigten). Hierbei zeigt sich, dass große ostdeutsche Unternehmen seltener FuE-Dienstleistungen nachfragen als große westdeutsche Betriebe.

Tabelle 5.19: Beteiligung an Vertragsforschung nach Regionen (alte/neue Bundesländer)

\begin{tabular}{lcccc}
\hline \multirow{2}{*}{ Regionen } & \multirow{2}{*}{ insgesamt } & \multicolumn{3}{c}{ Betriebsgrößenklasse } \\
& & bis 49 & $50-249$ & über 250 \\
\hline alte Bundesländer & 39,6 & 27,1 & 34,2 & 59,6 \\
neue Bundesländer & 39,5 & 34,8 & 43,4 & 50,0 \\
\hline \hline
\end{tabular}

Quelle: MIP 2003, eigene Auswertung.

\subsubsection{Auftragsvergabe nach Wirtschaftszweigen der Unterneh- men}

Die Auswertung nach Branchen lässt folgende Schlüsse zu (vgl. Tab. 5.20). ${ }^{484}$ Hohe Anteile der Unternehmen, die auf das Instrument der Vertragsforschung zugreifen, können insbesondere in den Branchen festgestellt werden, die nach der Klassifikation von Pavit ${ }^{485}$ dem wissensbasierten Sektor und dem Sektor der Spezialanbieter zugeordnet werden (chemische Industrie, Glasgewerbe und

484 Die Ergebnisse für den Bergbau werden aufgrund der geringen Beobachtungszahl $(n=13)$ nicht berücksichtigt.

485 Vgl. Abschnitt 3.4.3.1, S. 89. 
Keramik, Maschinenbau, EDV, Nachrichten-, Elektro-, Messtechnik und Optik). Dies sind zugleich diejenigen Branchen des Verarbeitenden Gewerbes, in denen in Deutschland hohe Anteile von innovierenden Unternehmen festgestellt werden und die durch relativ hohe Forschungsintensitäten gekennzeichnet sind.

Den höchsten Anteil der FuE-Aufträge erteilenden Unternehmen verzeichnet mit knapp $69 \%$ der Fahrzeugbau, eine Branche, für die nach den Ergebnissen der Wissenschaftsstatistik für das Jahr 1999 auch die relativ höchste Bedeutung der externen FuE-Dienstleistungen festgestellt wurde (mit einem 22,4\%-igem Anteil der externen FuE-Aufwendungen an den FuEGesamtaufwendungen). Unter den innovierenden Unternehmen der Lebensmittelindustrie greifen über $40 \%$ auf das Instrument der Vertragsforschung zurück. Diese beiden Branchen können dem skalenintensiven Sektor zugeordnet werden, einer Gruppe, die ihren komparativen Vorteil laut Pavitt auf die Forschungsanstrengungen der Zulieferer stützt.

Lediglich knapp 30\% der Unternehmen der betrachteten Dienstleistungsgruppen (Datenverarbeitung und Datenbanken, FuE, Dienstleistungen für Unternehmen) vergeben FuE-Aufträge an unternehmensexterne Partner. Ein Anteil von $30,8 \%$ der auftragserteilenden Unternehmen des Dienstleistungssektors wurde ebenfalls in der Stichprobe des MIP 1993 festgestellt. Damit sind Unternehmen der wissensintensiven Dienstleistungsbranchen nicht überdurchschnittlich häufig an der Auftragsvergabe beteiligt; sie bieten jedoch verstärkt FuE-Dienstleistungen an: Eine zusätzliche Auswertung der Daten des MIP 1993 zeigt, dass über die Hälfte $(53,4 \%)$ dieser Unternehmen FuEDienstleistungen für Dritte durchführt.

Schließlich sind innovierende Unternehmen der durch niedrige Forschungsintensität gekennzeichneten Branchen wie Textil- und Bekleidungsgewerbe, Holzund Papier- und Verlagsgewerbe und Herstellung von Möbel, Schmuck, Musikinstrumenten, Sportgeräten und Spielwaren relativ selten (mit Anteilen von bis $20 \%$ ) an der Forschungsvergabe beteiligt.

\subsubsection{Determinanten der Auftragsvergabe}

In den nachfolgenden Schätzungen der Bestimmungsfaktoren der Auftragsvergabe wird als abhängige Variable eine Dummy-Variable verwendet, die auf der Angabe der Unternehmen darüber basiert, ob sie in den vergangenen zwei Jahren externe FuE-Aktivitäten durchgeführt haben, d.h. ob FuE-Aufträge an Dritte vergeben wurden. Diese Variable nimmt den Wert $1(0)$ bei einer positiven (negativen) Antwort auf diese Frage an. 
Tabelle 5.20: Beteiligung an Vertragsforschung nach Wirtschaftszweigen der Unternehmen

\begin{tabular}{lc}
\hline \hline Wirtschaftszweige (nach WZ 2003) & $\begin{array}{c}\text { Anteil } \\
\text { der Unternehmen }\end{array}$ \\
\hline Ernährung/Tabak (15-16) & 40,7 \\
Textil-/Bekleidung-/Ledergewerbe (17-19) & 12,8 \\
Holz-/Papier-/Verlags-/Druckgewerbe (20-22) & 19,3 \\
Chemische Industrie (23-24) & $\mathbf{5 5 , 9}$ \\
Gummi- und Kunststoffe (25) & 23,5 \\
Glasgewerbe, Keramik (26) & $\mathbf{5 1 , 0}$ \\
Herstellung von Metallen & \\
und Metallerzeugnissen (27-28) & 36,8 \\
Maschinenbau (29) & $\mathbf{5 2 , 2}$ \\
EDV, Elektro- und Nachrichtentechnik (30-32) & $\mathbf{5 2 , 9}$ \\
MMSR, Optik (33) & $\mathbf{5 8 , 3}$ \\
Fahrzeugbau (34-35) & $\mathbf{6 8 , 7}$ \\
Herstellung von MSMSS (36) & 15,0 \\
Recycling, Elektrizitäts- und Wasserversorgung, & \\
Abwasser und Abfallbeseitigung (37, 40-41, 90) & 39,4 \\
Baugewerbe & 23,5 \\
Datenverarbeitung und Datenbanken, FuE, & \\
Dienstleistungen für Unternehmen (72-74) & 29,4 \\
\hline \hline
\end{tabular}

Quelle: MIP 2003, eigene Auswertung. 


\subsubsection{Spezifikation der Determinanten}

Die in der Schätzung der Determinanten der Auftragsvergabe verwendeten unabhängigen Variablen sind bereits in Abschnitt 5.3.4.1 in Beziehung zu den Hypothesen erläutert worden. Daher wird an dieser Stelle nur auf die Unterschiede bei der Spezifikation einzelner Variablen eingegangen. Eine Zusammenstellung der verwendeten Variablen befindet sich in Tab. 5.21.

Im Fragebogen der Erhebung 2003 wurden die Unternehmen nicht gebeten, auf einer 5-stufigen Skala die Bedeutung der externen Wissensquellen einzuschätzen. Stattdessen wurden sie danach gefragt, ob Kunden, Wettbewerber, Wissenschaft und Lieferanten eine Innovationsquelle darstellen. In der Schätzung werden zur Beschreibung der technologischen Möglichkeiten die Antworten auf diese Frage verwendet, kodiert als Dummy-Variablen mit der Ausprägung 1 bei einer positiven Antwort.

Die Frage zur Bedeutung einzelner Hemmnisfaktoren der Innovationstätigkeit wurde ebenfalls umformuliert. Gefragt wurde lediglich, ob hohes wirtschaftliches Risiko bzw. hohe Innovationskosten zur Verlängerung von Projektlaufzeiten und zum Abbruch von Innovationsprojekten bzw. zum Verzicht auf Innovationsaktivitäten geführt haben oder ob diese Faktoren bei den Innovationsentscheidungen nicht relevant waren. Unter Anwendung dieser Angaben wurden zwei Dummy-Variablen gebildet (d_kosten und d_risiko). Sie nehmen den Wert 1 ein, wenn hohe Innovationskosten bzw. hohes Innovationsrisiko entscheidungsrelevant waren.

Im Fragebogen der Erhebung aus dem Jahr 2003 wurden die Unternehmen ebenfalls nicht nach der subjektiven Einschätzung der Umsatzentwicklung gefragt. Allerdings stehen in diesem Datensatz Angaben zum Umsatzniveau in den letzten drei Jahren zur Verfügung. Diese Angaben wurden herangezogen, um eine ordinalskalierte Variable zu bilden, die die Umsatzentwicklung der jeweiligen Unternehmen im Zeitraum zwischen 2000 und 2002 beschreibt. Sie nimmt folgende Werte in Abhängigkeit von der Umsatzentwicklung an:

- 1 für Innovatoren, deren Umsatz im betrachteten Zeitraum um mehr als $7 \%$ gesunken ist,

- 2 für Innovatoren, deren Umsatzentwicklung im Intervall zwischen -7\% und $+5 \%$ lag,

- 3 für Innovatoren, deren Umsatz zwischen $5 \%$ und $22 \%$ gestiegen ist, und 
Tabelle 5.21: Spezifikation der Regressoren (MIP 2003)

\begin{tabular}{|c|c|c|c|}
\hline Variable & Erläuterung & MW & $\mathrm{SD}$ \\
\hline & Unternehmenseigenschaften & & \\
\hline bgk1 & Betriebsgrößenklasse: bis 50 Beschäftigte (Dummy) & 0,45 & 0,50 \\
\hline bgk3 & Betriebsgrößenklasse: über 250 Beschäftigte (Dummy) & 0,24 & 0,43 \\
\hline \multirow[t]{2}{*}{ ugrup } & Unternehmen ist ein Teil einer Unternehmensgruppe & & \\
\hline & (Dummy) & 0,41 & 0,49 \\
\hline d_risiko & hohes Risiko ist ein Innovationshemmnis (Dummy) & 0,31 & 0,46 \\
\hline d_kosten & hohe Kosten sind ein Innovationshemmnis (Dummy) & 0,32 & 0,47 \\
\hline umwachs & Umsatzwachstum in vergangenen zwei Jahren $\left({ }^{*}\right)$ & 2,54 & 1,15 \\
\hline \multirow[t]{2}{*}{ expint } & Exportintensität (Exporte/Umsatz) & 0,22 & 0,26 \\
\hline & Bedeutung unternehmensexterner Wissensq & iellen & \\
\hline diwiss & Wissenschaft ist Innovationsquelle (Dummy) & 0,18 & 0,38 \\
\hline dikunden & Kunden sind Innovationsquelle (Dummy) & 0,61 & 0,49 \\
\hline d_iwettb & Wettbewerber sind Innovationsquelle (Dummy) & 0,24 & 0,43 \\
\hline \multirow[t]{2}{*}{ dizulief } & Lieferanten sind Innovationsquelle (Dummy) & 0,21 & 0,41 \\
\hline & Absorptionsfähigkeit & & \\
\hline \multirow[t]{2}{*}{ fuekon } & kontinuierliche FuE (Dummy) & 0,58 & 0,49 \\
\hline & Erfahrung & & \\
\hline \multirow[t]{3}{*}{ koop } & Kooperationstätigkeit im Innovationsbereich & & \\
\hline & (Dummy) & 0,46 & 0,50 \\
\hline & Sonstige Variablen & & \\
\hline ost & Unternehmen aus neuen Bundesländern (Dummy) & 0,34 & 0,48 \\
\hline
\end{tabular}

* Ordinalskalierte Variable mit fünf Kategorien.

Quelle: Eigene Darstellung. 
- 4 für Innovatoren, deren Umsatz um mehr als $22 \%$ gestiegen ist.

Die Unter- und Obergrenzen für die Kodierung wurden so gewählt, dass auf jede Ausprägung der Variable umwachs die gleiche Anzahl der Unternehmen entfällt, d.h. es wurden Quantile der Verteilung der Umsatzwachstumsraten verwendet.

Bei den Variablen bgk1, bgk3, ugrup, fuekon, expint, koop und ost bestehen keine Unterschiede zu der Spezifikation in Abschnitt 5.3.4.1. Dies wird zustätzlich durch die Verwendung der gleichen Variablennamen hervorgehoben.

Einige der Variablen, die in der Schätzung der Determinanten der Auftragsvergabe in Abschnitt 5.3.4.1 verwendet wurden, können in der Schätzung für das Jahr 2003 nicht berücksichtigt werden, da entsprechende Informationen in der Erhebung von 2003 nicht ermittelt wurden. Erstens wird für die Wirksamkeit der Schutzmechanismen nicht kontrolliert. Da sich der Einfluss dieser Variable als nicht signifikant erwiesen hat, stellt dies keine weitreichende Einschränkung dar. Allerdings kann damit für eine mögliche Änderung in der Einstellung der Unternehmen zum Schutz eigener Wettbewerbsvorteile nicht kontrolliert werden. Ferner wurden im Jahr 2003 keine Daten über Umsatzanteile der wichtigsten Produktgruppen erhoben, die Variable divers wird in der Folge ebenfalls nicht berücksichtigt. Auch diese Variable zeigte sich jedoch in der Schätzung anhand von Daten des MIP 1993 als nicht signifikant. Leider kann aufgrund von fehlenden Informationen nicht für den Einfluss von Wettbewerbsbedingungen kontrolliert werden.

\subsubsection{Schätzergebnisse}

Die Ergebnisse der Schätzungen von drei verschiedenen Logit-Modellen sind in Tab. 5.22 aufgelistet. Erneut wurde eine Schätzung mit Branchendummies (Modell 3A), eine Schätzung ohne Branchendummies (Modell 3B) und eine Schätzung unter zusätzlicher Berücksichtigung der Variable ugrup (Modell 3C) durchgeführt. Die Unterschiede in BIC lassen dem letzten Modell mit der Variable ugrup den Vorzug geben. Eine Schätzung des Modells 3C mit Branchendummies führte zu keinen Unterschieden in den Vorzeichen und der Signifikanz einzelner Koeffizienten mit einer Ausnahme: Wie im Modell 3A erwies sich der Einfluss der Variable expint als nicht signifikant. Schätzungen mit dem Probit-Modell führten zu keinen abweichenden Ergebnissen im Hinblick auf Vorzeichen und Signifikanz der Variablen. 
Tabelle 5.22: Schätzergebnisse der Determinanten der Auftragsvergabe (MIP 2003)

\begin{tabular}{|c|c|c|c|c|c|c|}
\hline \multirow[b]{2}{*}{ Variable } & \multicolumn{2}{|c|}{ Modell 3A } & \multicolumn{2}{|c|}{ Modell 3B } & \multicolumn{2}{|c|}{ Modell 3C } \\
\hline & Koeff. & $\mathrm{t}$-Werte & Koeff. & t-Werte & Koeff. & t-Werte \\
\hline Konstante & $-1,64$ & $-1,50$ & $-2,15^{* * *}$ & $-9,05$ & $-2,39 * * *$ & $-9,62$ \\
\hline \multicolumn{7}{|c|}{ Unternehmenseigenschaften } \\
\hline bgk1 & $-0,15$ & $-0,83$ & $-0,29^{*}$ & $-1,70$ & $-0,12$ & $-0,69$ \\
\hline bgk3 & $0,45^{* *}$ & 2,32 & $0,54^{* * *}$ & 2,97 & $0,43^{* *}$ & 2,30 \\
\hline ugrup & & & & & $0,52^{* * *}$ & 3,22 \\
\hline d_risiko & $-0,10$ & $-0,49$ & $-0,09$ & $-0,45$ & $-0,12$ & $-0,56$ \\
\hline d_kosten & $0,56^{* * *}$ & 2,62 & $0,55^{* * *}$ & 2,71 & $0,56^{* * *}$ & 2,68 \\
\hline umwachs & 0,02 & 0,35 & 0,03 & 0,43 & 0,02 & 0,39 \\
\hline expint & 0,38 & 1,23 & $0,62^{* *}$ & 2,23 & $0,54^{*}$ & 1,93 \\
\hline \multicolumn{7}{|c|}{ Bedeutung unternehmensexterner Wissensquellen } \\
\hline d_iwiss & $0,48^{* *}$ & 2,39 & $0,49^{* *}$ & 2,56 & $0,48^{* *}$ & 2,47 \\
\hline dikunden & 0,02 & 0,12 & 0,06 & 0,42 & 0,06 & 0,40 \\
\hline d jwettb & 0,09 & 0,55 & 0,12 & 0,74 & 0,08 & 0,50 \\
\hline d_izulief & $-0,06$ & $-0,35$ & $-0,15$ & $-0,83$ & $-0,12$ & $-0,72$ \\
\hline \multicolumn{7}{|c|}{ Absorptionsfähigkeit } \\
\hline fuekon & $0,91^{* * *}$ & 5,66 & $0,94^{* * *}$ & 6,10 & $0,96^{* * *}$ & 6,17 \\
\hline \multicolumn{7}{|l|}{ Erfahrung } \\
\hline koop & $1,15^{* * *}$ & 7,27 & $1,11^{* * *}$ & 7,61 & $1,11^{* * *}$ & 7,51 \\
\hline \multicolumn{7}{|c|}{ Sonstige Variablen } \\
\hline ost & 0,25 & 1,57 & $0,30^{*}$ & 1,95 & $0,31^{* *}$ & 2,02 \\
\hline Branchendummies & \multicolumn{2}{|c|}{ ja } & \multicolumn{2}{|c|}{ nein } & \multicolumn{2}{|c|}{ nein } \\
\hline $\mathbf{n}$ & \multicolumn{2}{|c|}{1150} & \multicolumn{2}{|c|}{1150} & \multicolumn{2}{|c|}{1150} \\
\hline Log-Likelihood & \multicolumn{2}{|c|}{$-606,80$} & \multirow{2}{*}{\multicolumn{2}{|c|}{$-638,44$}} & \multicolumn{2}{|c|}{$-633,11$} \\
\hline \multicolumn{5}{|l|}{ Likelihood-Ratio- } & & \\
\hline$\chi^{2}$ & \multicolumn{2}{|c|}{250,30} & \multirow{2}{*}{\multicolumn{2}{|c|}{213,45}} & \multicolumn{2}{|c|}{222,54} \\
\hline \multicolumn{5}{|l|}{ McFaddens } & & \\
\hline Pseudo- $r^{2}$ & \multicolumn{2}{|c|}{0,22} & \multicolumn{2}{|c|}{0,18} & \multicolumn{2}{|c|}{0,18} \\
\hline $\mathrm{BIC}$ & \multicolumn{2}{|c|}{$-6686,67$} & \multicolumn{2}{|c|}{$-6729,10$} & \multicolumn{2}{|c|}{$-6732,71$} \\
\hline
\end{tabular}

Huber/White-Schätzer der Varianz; *** Signifikant auf dem $1 \%$ Niveau; ${ }^{* *}$ signifikant auf dem $5 \%$ Niveau; *signifikant auf dem $10 \%$ Niveau.

Quelle: MIP 2003, eigene Auswertung. 
Der Likelihood-Ratio-Test lässt die Hypothese verwerfen, dass alle Koeffizienten außer der Konstanten gleich Null sind. Der Wert für McFaddens-Pseudo- $r^{2}$ für die beiden Modelle ohne Branchendummies (3B und 3C) liegt bei 0,18 und damit niedriger als in der Schätzung anhand von Daten der ersten Welle des Mannheimer Innovationspanels (vgl. Tab. 5.14).

Bei der Erläuterung der Schätzergebnisse soll im Folgenden explizit auf Ähnlichkeiten und Unterschiede $\mathrm{zu}$ den Befunden des Abschnittes 5.3.4.2 eingegangen werden.

In der Schätzung der Determinanten der Auftragsvergabe anhand von Daten des MIP 2003 zeigt sich erneut ein signifikanter Betriebsgrößeneffekt. Wird für die Zugehörigkeit zu einer Unternehmensgruppe kontrolliert (Modell 3C), dann verliert der Koeffizient bei der Variable bgk1 an Signifikanz. Im Unterschied zu der Schätzung in Abschnitt 5.3.4.2 übt jedoch die Zugehörigkeit zur Unternehmensgruppe einen signifikant positiven Einfluss aus auf die Neigung der Unternehmen, FuE-Verträge an Dritte zu erteilen. ${ }^{486}$ Verantwortlich für die Unterschiede in den Schätzergebnissen bezüglich der Variable ugrup können die in dem betrachteten Zeitraum stattgefundenen Ausgliederungen der FuEEinheiten in rechtlich selbstständige Unternehmen sein. Wie bereits erläutert, gaben in der ifo-Befragung $5 \%$ der Unternehmen an, ihre FuE-Kapazitäten zwischen 1997 und 2000 in eigenständige FuE-Tochtergesellschaften verlagert zu haben; $7,3 \%$ der Unternehmen planten eine Ausgliederung von FuEGesellschaften.

Der Einfluss der subjektiven Einschätzung über das Risiko der Innovationstätigkeit sowie der Einfluss der Umsatzentwicklung verliert in dieser Schätzung an Signifikanz. Der Koeffizient bei der Variable $d \_k o s t e n$, positiv aber insignifikant bei der Schätzung in Abschnitt 5.3.4.2, wird in der aktuellen Schätzung signifikant. Erneut steigt mit der Exportintensität der Unternehmen die Wahrscheinlichkeit für die Auftragsvergabe.

Die Ergebnisse zum Einfluss der technologischen Möglichkeiten erweisen sich als vergleichsweise robust. Signifikant positiver Einfluss geht von der DummyVariable aus, die die Bedeutung der Wissenschaft als eine Innovationsquelle erfasst. Der Einfluss des Wissens von Kunden und Wettbewerbern übt keinen signifikanten Einfluss aus. Im Unterschied zu der früheren Schätzung verliert lediglich der (negative) Koeffizient bei der Variable d_izulief an Signifikanz.

${ }^{486}$ Ein signifikant positiver Effekt der Zugehörigkeit zur Unternehmensgruppe wurde auch in der finnischen Studie festgestellt, vgl. Leiponen (2002), S. 273. 
Absorptionsfähigkeit ist weiterhin eine notwendige Voraussetzung für die Übernahme von im Rahmen von Vertragsforschungsbeziehungen erarbeitetem Wissen. Der Koeffizient bei der Variable fuekon ist in jedem Modell signifikant positiv. Kooperierende Unternehmen haben erneut eine über 3-fach höhere Chance $\left(e^{1,11}=3,03\right)$, FuE-Verträge zu erteilen als Innovatoren ohne Kooperationserfahrung.

Bei der multivariaten Betrachtung - d.h. insbesondere unter Kontrolle für Unternehmenseigenschaften - zeigt sich in beiden Schätzungen (anhand von Daten des MIP 1993 und des MIP 2003), dass ostdeutsche Unternehmen im Vergleich $\mathrm{zu}$ westdeutschen Unternehmen eine 1,3-fache Chance $\left(e^{0,31}=1,36\right)$ haben, FuE-Aufträge an Dritte zu erteilen.

Schätzungen unter Ausschluss der wissensintensiven Branchen des Dienstleistungssektors

Die Schätzungen unter Ausschluss der wissensintensiven Branchen des Dienstleistungssektors $(n=820)$ führen nur zu geringfügigen Unterschieden. Die Variablen, die sowohl in der Schätzung anhand von Daten des MIP 1993 als auch in den vorangegangenen Schätzungen anhand des Datensatzes des MIP 2003 (mit allen Beobachtungen) hochsignifikant waren, zeigen sich weiterhin signifikant. Ein positiver Einfluss auf die Wahrscheinlichkeit der Auftragsvergabe geht sowohl von der internen Absorptionsfähigkeit (Variable fuekon), der Kooperationserfahrung (koop) als auch von den Dummy-Variablen bgk3, ugrup und iwiss aus.

Unterschiede ergeben sich im Hinblick auf die Signifikanz von vier Variablen: Die Koeffizienten bei bgk1, d_kosten, expint und ost verlieren an Signifikanz. Die Ergebnisse bezüglich der Variable $d \_k o s t e n$ lassen die Vermutung zu, dass die kostenorientierte Auftragsvergabe eine besondere Rolle für Unternehmen der wissensintensiven Dienstleistungsbranchen spielen kann. Die Unternehmen der wissensintensiven Dienstleistungsbranchen prägen zudem offensichtlich die Schätzergebnisse bezüglich der Variable ost.

Zugleich steigt in der Schätzung ohne wissensintensive Dienstleister die Einflussstärke der Variablen umwachs und izulief. Der negative Koeffizient bei der Variable d_izulief verfehlt im Modell 3B nur knapp das Signifikanzniveau von $10 \%(\mathrm{p}=0,108)$. 
Tabelle 5.23: Schätzergebnisse der Determinanten der Auftragsvergabe (MIP 2003, ohne wissensintensive Branchen des Dienstleistungssektors)

\begin{tabular}{|c|c|c|c|c|c|c|}
\hline \multirow[b]{2}{*}{ Variable } & \multicolumn{2}{|c|}{ Modell 4A } & \multicolumn{2}{|c|}{ Modell 4B } & \multicolumn{2}{|c|}{ Modell 4C } \\
\hline & Koeff. & t-Werte & Koeff. & $\mathrm{t}$-Werte & Koeff. & t-Werte \\
\hline Konstante & $-1,42$ & $-1,37$ & $-1,99 * * *$ & $-7,93$ & $-2,21^{* * *}$ & $-7,85$ \\
\hline \multicolumn{7}{|c|}{ Unternehmenseigenschaften } \\
\hline bgk1 & $-0,33$ & $-1,50$ & $-0,30$ & $-1,43$ & $-0,15$ & $-0,68$ \\
\hline bgk3 & $0,55^{* * *}$ & 2,63 & $0,57^{* * *}$ & 2,93 & $0,48^{* *}$ & 2,36 \\
\hline ugrup & & & & & $0,50^{* * *}$ & 2,71 \\
\hline d_risiko & $-0,05$ & $-0,18$ & $-0,05$ & $-0,19$ & $-0,06$ & $-0,23$ \\
\hline d_kosten & 0,25 & 0,99 & 0,31 & 1,28 & 0,32 & 1,28 \\
\hline umwachs & 0,08 & 0,96 & 0,09 & 1,24 & 0,08 & 1,14 \\
\hline expint & 0,09 & 0,24 & 0,13 & 0,41 & 0,06 & 0,19 \\
\hline \multicolumn{7}{|c|}{ Bedeutung unternehmensexterner Wissensquellen } \\
\hline d_iwiss & $0,87^{* * *}$ & 3,04 & $0,92^{* * *}$ & 3,57 & $0,91^{* * *}$ & 3,49 \\
\hline d_ikunden & $-0,01$ & $-0,05$ & 0,06 & 0,33 & 0,05 & 0,28 \\
\hline d_iwettb & 0,12 & 0,59 & 0,14 & 0,72 & 0,10 & 0,52 \\
\hline dizulief & $-0,21$ & $-0,97$ & $-0,33$ & $-1,61$ & $-0,30$ & $-1,47$ \\
\hline \multicolumn{7}{|c|}{ Absorptionsfähigkeit } \\
\hline fuekon & $0,82^{* * *}$ & 4,26 & $0,93^{* * *}$ & 5,10 & $0,94^{* * *}$ & 5,12 \\
\hline \multicolumn{7}{|l|}{ Erfahrung } \\
\hline koop & $1,23^{* * *}$ & 6,70 & $1,28^{* * *}$ & 7,45 & $1,27^{* * *}$ & 7,34 \\
\hline \multicolumn{7}{|c|}{ Sonstige Variablen } \\
\hline ost & 0,03 & 0,14 & 0,04 & 0,22 & 0,07 & 0,36 \\
\hline Branchendummies & \multicolumn{2}{|c|}{ ja } & \multicolumn{2}{|c|}{ nein } & \multicolumn{2}{|c|}{ nein } \\
\hline $\mathrm{n}$ & \multicolumn{2}{|c|}{820} & \multicolumn{2}{|c|}{820} & \multicolumn{2}{|c|}{820} \\
\hline Log-Likelihood & \multirow{2}{*}{\multicolumn{2}{|c|}{$-425,53$}} & \multirow{2}{*}{\multicolumn{2}{|c|}{$-449,69$}} & \multicolumn{2}{|c|}{$-445,98$} \\
\hline Likelihood-Ratio- & & & & & \multirow{3}{*}{\multicolumn{2}{|c|}{176,21}} \\
\hline$\chi^{2}$ & \multirow{2}{*}{\multicolumn{2}{|c|}{197,90}} & \multirow{2}{*}{\multicolumn{2}{|c|}{170,99}} & & \\
\hline McFaddens & & & & & & \\
\hline Pseudo- $r^{2}$ & \multicolumn{2}{|c|}{0,24} & \multicolumn{2}{|c|}{0,20} & \multicolumn{2}{|c|}{0,21} \\
\hline $\mathrm{BIC}$ & \multicolumn{2}{|c|}{$-4462,72$} & \multicolumn{2}{|c|}{$-4508,32$} & \multicolumn{2}{|c|}{$-4509,03$} \\
\hline
\end{tabular}

Huber/White-Schätzer der Varianz; *** Signifikant auf dem $1 \%$ Niveau; ** signifikant auf dem $5 \%$ Niveau; ${ }^{*}$ signifikant auf dem $1 \%$ Niveau.

Quelle: MIP 2003, eigene Auswertung. 


\subsection{Diskussion der Ergebnisse}

In diesem Abschnitt sollen die zentralen Erkenntnisse der statistischen Analyse des Phänomens der Vertragsforschung zusammengefasst werden. Hierbei wird zunächst die empirische Evidenz zu den im theoretischen Teil der Arbeit formulierten Hypothesen ausführlich diskutiert. Anschließend wird auf einige besondere Ergebnisse der Analyse eingegangen.

Hypothese 1 Eine hohe Einschätzung der Effektivität von Mechanismen zum Schutz eigenen innovationsrelevanten Wissens begünstigt die Entscheidung für Vertragsforschung.

(nicht bestătigt)

Die ifo-Befragung im Verarbeitenden Gewerbe führte zwar zur Schlussfolgerung, dass die Gefahr des Know-how-Abflusses einer der wichtigsten Hemmnisfaktoren bei der Inanspruchnahme externer FuE-Dienstleistungen darstellt. Allerdings zeigte die hier vorliegende ökonometrische Analyse, dass die einzelwirtschaftlichen Einschätzungen über die im technologischen Umfeld einer Unternehmung vorherrschenden Appropriierungsbedingungen keinen signifikanten Einfluss sowohl auf die Wahrscheinlichkeit der Auftragsvergabe als auch auf die Wahrscheinlichkeit der Durchführung von FuE-Aufträgen ausüben. Unternehmen, die sich an den Vertragsforschungsaktivitäten beteiligen, sind demnach nicht zwangsläufig unter denjenigen Innovatoren zu finden, die die Effektivität von Mechanismen zum Schutz eigenen Wissens überdurchschnittlich hoch einschätzen.

Eine Erklärung für dieses Ergebnis kann unter Rückgriff auf die Postulate der industrieökonomischen Modelle der FuE-Kooperation gesucht werden. Bei ohnehin vorhandenen erheblichen Spilloverraten - so der Befund der Industrieökonomik - kann die Entscheidung für die horizontale Zusammenarbeit mit anderen Wirtschaftsakteuren und für die Offenlegung eigenen Wissens gewinnsteigernd wirken, wenn es dadurch zur Internalisierung der Externalitäten der FuE-Tätigkeit kommt. Die Entscheidungen der Unternehmen über eine Beteiligung an der Vertragsforschung könnte folglich als eine Abwägung zwischen Gefahren des einseitigen Wissensabflusses und Vorteilen der Internalisierung von Externalitäten der FuE-Tätigkeit aufgefasst werden.

Es ist jedoch auch eine alternative Erklärung für die Insignifikanz der Variablen möglich, die die Aneignungsbedingungen beschreiben. In der Industrieökonomik wird häufig von der Annahme ausgegangen, dass die Spilloverraten bei 
anwendungsnaher Forschung geringer ausfallen als bei FuE-Vorhaben, die auf die Erarbeitung genereller Wissensfortschritte abzielen. Nimmt man an, dass es sich bei den Vertragsforschungsbeziehungen um anwendungsnahe Vorhaben handelt - worauf zumindest die Untersuchungen der Vertragsforschungsaktivitäten in der Gruppe der Klein- und Mittelunternehmen schließen lassen, dann kann argumentiert werden, dass der insignifikante Einfluss der Variablen, die die Aneignungsbedingungen beschreiben, auf die spezifische Ausrichtung der über den Markt abgewickelten FuE-Projekte zurückzuführen ist, die gewährleistet, dass erhebliche Spilloverraten von den Unternehmen nicht erwartet werden.

Hypothese 2 Das in Kooperationsbeziehungen aufgebaute Vertrauen zum Transaktionspartner begünstigt die Entscheidung für die Vertragsforschung.

(bestätigt)

Der Einfluss der Variable koop erwies sich in allen geschätzten Modellen als hochsignifikant. Kooperierende Unternehmen haben eine 3,6-fache Chance, zur Gruppe der FuE-Dienstleister zu gehören (vgl. Modell 1B) und eine 3-fache Chance, FuE-Aufträge zu vergeben (vgl. Modelle 3B, 3C) als nicht kooperierende Unternehmen.

Hypothese 3 Auftragnehmer sind unter den Unternehmen zu erwarten, die über materielle (Ausrüstungsgegenstände) und immaterielle (distinktive Kompetenzen) Ressourcen verfügen, die nicht erst als transaktionsspezifische Investitionen aufgebracht werden müssen.

(bestätigt)

Bereits bei der Untersuchung der Motive der Auftragsvergabe zeigte sich, dass ein Zugriff auf Spezialwissen bzw. Know-how der Transaktionspartner sowie der Zugang zu spezieller technischer Ausrüstung die wesentlichen Faktoren bei der Entscheidung für einen externen Bezug von FuE-Leistungen bilden. Die vorliegende ökonometrische Analyse bestätigte diese Befunde. Es zeigte sich, dass kontinuierlich forschende Unternehmen, d.h. diejenigen Wirtschaftseinheiten, die ihre FuE-Kompetenzen in dauerhafter Auseinandersetzung mit der Lösung von technologischen Problemen aufbauen konnten, eine 4,1-fach höhere Chance haben, FuE-Aufträge zu attrahieren (vgl. Modell 1B).

Dieser Befund lässt die folgende Interpretation zu. Bei den Vertragsforschungsbeziehungen handelt es sich um homogene Transaktionsbeziehungen in dem 
Sinne, dass die Erbringung von transaktionsspezifischen Investitionen seitens des Vertragsforschungsanbieters nicht notwendig ist. Diese Notwendigkeit besteht jedoch deswegen nicht, weil die für die Erbringung einer bestimmten Leistung benötigten speziellen apparativen Vorrichtungen in der Vergangenheit angeschafft und das besondere Know-how von ausgewählten Unternehmen bereits angesammelt wurde. Nur aus der Sicht des Auftraggebers wären hochspezifische Investitionen notwendig, um die benötigte FuE-Leistung intern zu erbringen.

Dies impliziert zugleich, dass die Untersuchung des Phänomens der Vertragsforschung nicht im statischen Analyserahmen erfolgen sollte, sondern dass bei der Auseinandersetzung mit den Organisationsentscheidungen im FuE-Bereich der Problematik der Kumulativität der Wissenserzeugung explizit Rechnung getragen werden muss. Die Entscheidungen der Wirtschaftsakteure bezüglich der Inanspruchnahme externer FuE-Dienstleistungen werden offensichtlich bei Berücksichtigung der zum jeweiligen Zeitpunkt extern bereits vorhandenen innovationsrelevanten Ressourcen materieller und immaterieller Art getroffen. Ist bestimmtes Fachwissen von einem unternehmensexternen Partner in der Vergangenheit in kontinuierlicher Auseinandersetzung mit bestimmten technologischen Problemstellungen aufgebaut worden, dann verzichten ausgewählte Unternehmen auf die erneute Erarbeitung dieser Wissensfortschritte und verlassen sich bei neuen, verwandten FuE-Vorhaben auf die bereits vorhandenen Kompetenzen der externen Partner.

Hypothese 4 Die Vertragsforschung stellt ein kostengünstiges institutionelles Arrangement dar im Kontext von Aulastungsschwankungen und Engpassüberwindung.

(bestätigt)

Sowohl die Untersuchung der Motive der im Rahmen der Fördermaßnahme "Externe Vertragsforschung" geförderten kleinen und mittleren Unternehmen, als auch die Analyse der Motive der Auftragsvergabe in der gesamten Population der Unternehmen des Verarbeitenden Gewerbes (ifo-Studie) zeigte, dass die zeitliche Auslastung des eigenen FuE-Personals einen wesentlichen Einflussfaktor der Auftragsvergabe darstellt. In der ifo-Studie gaben auch über $72 \%$ der befragten FuE-Dienstleister an, dass „bessere Auslastung interner Kapazitäten" einen der Gründe für die Durchführung von FuE-Leistungen für Dritte darstellt. Die Schätzungen anhand von Daten des MIP 1993 bestätigten diese Befunde für die Gruppe der Auftraggeber (Modelle 2A-2C). Die 
Erwartung einer positiven Umsatzentwicklung begünstigt demnach die Entscheidung für Auftragsvergabe: Können schnell wachsende Unternehmen ihre interne FuE-Kapazitäten nicht zeitgleich aufbauen, so greifen sie auf das Instrument der Vertragsforschung zurück. In der Schätzung anhand von Daten des MIP 2003 übte die Variable umwachs zwar keinen signifikanten Einfluss auf die Wahrscheinlichkeit der Auftragsvergabe aus (Modelle 3A-3C). Allerdings kann dieses Ergebnis unter Umständen auf die Konstruktion des Indikators umwachs zurückgeführt werden, dem nicht mehr die subjektiven Einschätzungen der Unternehmen über das Unternehmenswachstum zugrunde lagen ${ }^{487}$, sondern bei dem eine branchenunabhängige Kodierung anhand der Quantile der Verteilung der Umsatzwachstumsraten verwendet wurde.

Hypothese 5 Die Vertragsforschung spielt für Unternehmen der Spitzentechnologie eine wichtige Rolle als Instrument der Sondierung von neuen technologischen Entwicklungen.

(bestätigt)

Die Sonderauswertung der Wissenschaftsstatistik zeigt, dass insbesondere in denjenigen Branchen des Verarbeitenden Gewerbes ein erheblicher Anteil der gesamten FuE-Ausgaben der Unternehmen für die externe FuE-Leistungen aufgewandt wird, die zugleich zu den forschungsintensiven Branchen der deutschen Wirtschaft gehören. Die bivariate Betrachtung anhand von Daten des MIP 2003 bestätigt für diese Branchen auch eine besonders starke Verbreitung des Instruments der Vertragsforschung: Über die Hälfte der innovierenden Unternehmen, die den Wirtschaftszweigen chemische Industrie, Glasgewerbe und Keramik, Maschinenbau, EDV, Nachrichten-, Elektro-, Messtechnik, Optik und Fahrzeugbau zugerechnet werden können, greift in ihrer Innovationsstrategie auf extern erbrachte FuE-Dienstleistungen zurück. Ferner zeigen die Schätzungen sowohl anhand von Daten des MIP 1993 als auch anhand von Daten des MIP 2003, dass eine hohe Einschätzung der Bedeutung der wissenschaftlichen Wissensquellen (öffentlich und industriefinanzierten Forschungseinrichtungen, Universitäten, Fachhochschulen, Technologietransferstellen und Patentschriften) die Entscheidung für die Vergabe von FuE-Aufträgen begünstigt (Modelle $2 \mathrm{~A}-2 \mathrm{C}, 3 \mathrm{~A}-3 \mathrm{C}, 4 \mathrm{~A}-4 \mathrm{C})$.

487 Diese Informationen wurden in der ersten Welle des MIP erhoben, nicht jedoch in der Erhebung 2003. 
Hypothese 6 Die Auftragsvergabe wird in der Gruppe der Großunternehmen als ein Instrument zur Bewältigung interner Koordinationsprobleme eingesetzt.

(bestätigt)

Die dominierende Rolle der Großunternehmen auf der Nachfrageseite des FuEDienstleistungsmarktes ist insbesondere in der Sonderauswertung der Wissenschaftsstatistik herausgestellt worden. Auch die ifo-Studie bestätigte, dass Großunternehmen signifikant häufiger das Instrument der Vertragsforschung in ihre Innovationsstrategie systematisch integrieren. Bei der bivariaten Analyse anhand von Daten des MIP 2003 wurde deutlich, dass größere Unternehmen signifikant häufiger FuE-Aufträge vergeben. In den Schätzungen zeigte sich ebenfalls, dass Unternehmen mit über 250 Beschäftigten signifikant höhere Chancen haben, zur Gruppe der Nachfrager von FuE-Dienstleistungen zu gehören - und dies auch dann, wenn für die Zugehörigkeit zur Unternehmensgruppe kontrolliert wurde (Modelle 2A-2C, 3A-3C, 4A-4C).

Hypothese 7 Interne FuE-Kompetenz begünstigt die Entscheidung für die Auftragsvergabe.

(beståtigt)

Bereits in der bivariaten Betrachtung wurde deutlich, dass das Instrument der Vertragsforschung insbesondere von denjenigen Unternehmen benutzt wird, die durch höhere Forschungsintensitäten (von über 3,5\%) gekennzeichnet sind. Dies liefert einen Hinweis darauf, dass der externe Bezug von FuEDienstleistungen mehrheitlich zur Ergänzung bzw. Stärkung der vorhandenen FuE-Kapazitäten herangezogen wird und nicht angesichts fehlender unternehmensinterner Forschungskompetenzen verwendet werden muss - ein Befund, der bereits bei der Betrachtung der im Rahmen der Maßnahme „Externe Vertragsforschung" geförderten Unternehmen zutage kam. Eine Erklärung für die offensichtlich komplementäre Inanspruchnahme der Vertragsforschung könnte in der von der industrieökonomischen Literatur betonten Rolle der Absorptionsfähigkeit liegen: vgl. hierzu die Diskussion der Evidenz zur Hypothese 10.

Hypothese 8 Vertragsforschungsbeziehungen können in der Folge einer Ausgliederung von FuE-Gesellschaften entstehen.

(bestătigt) 
In der Schätzung anhand von Daten des MIP 1993 zeigte sich zwar ein insignifikanter Einfluss der Zugehörigkeit zur Unternehmensgruppe sowohl auf die Wahrscheinlichkeit der Durchführung von FuE-Leistungen (Modell 1C) als auch auf die Wahrscheinlichkeit der Auftragsvergabe (Modell 2C). Ein signifikant positiver Einfluss der Zugehörigkeit zur Unternehmensgruppe ist dagegen in der Schätzung anhand von Daten des MIP 2003 erzielt worden (Modelle $3 \mathrm{C}$ und $4 \mathrm{C}$ ). Eine Erklärung für die Unterschiede kann in der stattgefundenen Welle der Ausgliederungen der rechtlich selbstständigen FuE-Gesellschaften im Zeitraum zwischen 1997 und 2003 gesucht werden, von der die ifo-Erhebung im Verarbeitenden Gewerbe berichtet. Auch die Wissenschaftsstatistik formuliert bei der Betrachtung von Ergebnissen der Sonderauswertung die Einschätzung, dass ein erheblicher Teil der Vertragsforschungsbeziehungen auf konzerninterne Verflechtungen zurückgeführt werden kann.

Hypothese 9 Die Vertragsforschung wird bei einer großen Bedeutung des Humankapitals unternehmensexterner Partner erwartet. (bestätigt)

Die Schätzungen zeigten, dass die Wahrscheinlichkeit der Durchführung von FuE-Leistungen mit der Humankapitalintensität der Unternehmen steigt (Modelle 1A-1C). Die Relevanz des Humankapitals wird auch durch die Ergebnisse einer US-amerikanischen Studie bestätigt, die zu der Schlussfolgerung kommt, dass „insourcing laboratories are more skill intensive than avarage“.

Hypothese 10 Für die Auftragsvergabe stellt die interne Absorptionsfähigkeit eine notwendige Bedingung dar.

(bestätigt)

Die grundlegende Bedeutung der Absorptionsfähigkeit bestätigt sich durchgehend in der multivariaten Analyse: Von der Variable fuekon geht unabhängig von der Spezifikation des empirischen Modells und dem Zeitpunkt der Betrachtung ein signifikant positiver Einfluss auf die Wahrscheinlichkeit der Auftragsvergabe aus (Modelle 2A-2C, 3A-3C, 4A-4C).

Nachfolgend soll stichpunktartig auf einige besondere Ergebnisse der empirischen Analyse hingewiesen werden:

- Erstens führen die Ergebnisse der Befragungen von Unternehmen zu der Schlussfolgerung, dass dem Motiv einer mit der Auftragsvergabe antizipierten Kostensenkung nur eine vergleichsweise geringe Rolle zukommt. 
In der ifo-Befragung im Verarbeitenden Gewerbe wurde zudem deutlich, dass ein hoher Preis für externe FuE-Leistungen den am häufigsten genannten Hemmnisfaktor bei der Inanspruchnahme externer FuEDienstleistungen darstellt. In der multivariaten Analyse anhand von Daten des MIP 1993 bestätigt sich tendenziell dieses Ergebnis: Die Variable kosten übt keinen signifikanten Einfluss auf die Wahrscheinlichkeit der Auftragsvergabe aus (Modelle 2A-2C). Allerdings liefern die Ergebnisse der Schätzungen anhand von Daten des MIP 2003 einen Hinweis darauf, dass der Kostenfaktor bei der Entscheidung für die Auftragsvergabe in der Gruppe der Unternehmen der wissensintensiven Dienstleistungsbranchen eine größere Rolle spielen kann.

- Besonders interessante Hinweise auf eine stattfindende Entwicklung liefern die Ergebnisse über die Bedeutung von Zulieferern als einer unternehmensexternen Innovationsquelle. Die auf Daten der ersten Welle des Mannheimer Innovationspanels basierenden Schätzungen zeigten einen signifikant negativen Einfluss der steigenden Bedeutung des Wissens von Zulieferern auf die Wahrscheinlichkeit der Auftragsvergabe. ${ }^{488}$ Dieses Ergebnis lässt sich als ein Hinweis auf die veränderte Strategie derjenigen Unternehmen interpretieren, die ihre Innovationsaktivitäten auf Forschungsleistungen der Zulieferer stützen: Möglicherweise gehen sie dazu über, die in eigener Innovationstätigkeit benötigte FuE-Leistung der Zulieferer nicht direkt zu vergüten, sondern bei der Preissetzung für die bezogenen Produkte zu berücksichtigen. Damit werden die Entwicklungsrisiken auf die Zulieferer übertragen; die industriellen Kunden vermeiden ihrerseits die Probleme, die sich bei der Spezifizierung und Abwicklung von FuE-Verträgen ergeben.

- Die ökonometrische Analyse anhand von Daten der ersten Welle des Mannheimer Innovationspanels liefert ferner Hinweise auf die Gültigkeit der Hypothese, dass die Zunahme der Wettbewerbsintensität zu Veränderungen der Organisationsstruktur von privatwirtschaftlicher FuE führt. Sowohl in den Schätzungen der Determinanten der Durchführung von FuE-Leistungen (Modelle 1A-1C) als auch in den Schätzungen der Bestimmungsfaktoren der Auftragsvergabe (Modelle 2A-2C) zeigt sich ein signifikanter Einfluss der Variable, welche die subjektive Einschätzung

488 Auch in den Schätzungen anhand von Daten des MIP 2003 (unter Ausschluss der wissensintensiven Branchen des Dienstleistungssektors) war der Koeffizient bei der Variable izulief negativ, und verfehlte nur knapp das Signifikanzniveau von 10\%, vgl. Modell 4B. 
der jeweiligen Unternehmen bezüglich der erwarteten Wettbewerbsintensität beschreibt.

- Die Ergebnisse bezüglich des Zusammenhangs zwischen der Exporttätigkeit und der Neigung zur Auftragsvergabe erweisen sich in dieser Studie als nicht robust. Zwar zeigt sich in der Schätzung anhand von Daten des MIP 1993 ein hochsignifikant positiver Einfluss der Exportintensität von Unternehmen auf die Wahrscheinlichkeit der Auftragsvergabe (Modelle 2A-2C). Diese Ergebnisse stimmen überein mit den Resultaten einer Schätzung in der Population der finnischen Unternehmen. Allerdings zeigt sich der Einfluss der Exportintensität der Unternehmen in den Schätzungen anhand von Daten der letzten Welle des MIP nur dann als signifikant, wenn Unternehmen der wissensintensiven Dienstleitungsbranchen berücksichtigt werden. Die Beziehung zwischen der Exporttätigkeit und der Neigung zur Auftragsvergabe sollte folglich in weiteren Studien verifiziert werden.

- Die bivariate Betrachtung der gesamten Population der innovierenden Unternehmen mit Sitz in den alten bzw. neuen Bundesländern anhand von Daten des MIP 2003 ließ keine Unterschiede im Hinblick auf eine unterschiedliche Innovationsstrategie erkennen. Die Analyse unter Berücksichtigung unterschiedlicher Betriebsgrößenklassen zeigte jedoch, dass kleine ostdeutsche Betriebe (mit bis zu 250 Beschäftigten) häufiger als kleine westdeutsche Betriebe zur Auftragsvergabe neigen; ein umgekehrter Zusammenhang galt für die Gruppe der großen Unternehmen. Die Ergebnisse der Schätzungen, in denen für Unternehmensgröße explizit kontrolliert wurde, lieferten Hinweise darauf, dass ostdeutsche Betriebe signifikant häufiger zur Auftragsvergabe neigen (Modelle 2A-2B, 3B-3C). Empfehlenswert wäre folglich eine gezielte Untersuchung der Bestimmungsfaktoren der Vertragsforschung in der Subpopulation der kleineren Unternehmen mit Sitz in den neuen Bundesländern.

- Schließlich soll auf zwei weitere Ergebnisse der Untersuchung zu den Bestimmungsfaktoren der Durchführung von FuE-Leistungen eingegangen werden. Zum einen zeigte sich, dass Unternehmen, die FuEDienstleistungen anbieten, offensichtlich Finanzierungsbeschränkungen unterliegen und womöglich Vertragsforschung als ein Instrument zur Attrahierung zusätzlicher finanzieller Ressourcen nutzen. Zum anderen war nur in den Schätzungen der Determinanten der Durchführung von FuEAufträgen der Einfluss der Variable ikunden signifikant positiv. Dies kann 
als Bestätigung der Hypothese von Pisano interpretiert werden, nach der der Zugang zu nachgelagerten Kompetenzen der industriellen Kunden einen der wesentlichen Gründe für die Zusammenarbeit der forschungsintensiven Kleinunternehmen des Biotechnologiesektors mit den etablierten großen Unternehmen der Pharmaindustrie darstellte. Zwar galt die Hypothese von Pisano der Gruppe der Biotechnologieuniernehmen. Allerdings erscheint eine Verallgemeinerung dieser Hypothese auf die weiter gefasste Gruppe der spezialisierten Zulieferer industrieller Großkunden möglich. Eine verwandte Überlegung stellen auch die Autoren der ifoStudie an und stützen sie auf die Ergebnisse der Interviews mit Vertretern der Unternehmen des Verarbeitenden Gewerbes. Wie die Autoren der Studie anführen, wurde in den Unternehmensgesprächen deutlich, dass „Anbieter von FuE-Dienstleistungen, die selbst nicht produzieren, vor allem in der Serienproduktion erhebliche Wettbewerbsnachteile gegenüber produzierenden Firmen haben “489. Der komparative Nachteil der reinen FuE-Dienstleister begründet sich demnach in der fehlenden Erfahrung bei der Überführung von prototypischen Entwicklungen in die Serienproduktion.

489 Koschatzky/Reinhard/Grenzmann (2003), S. 155 f. 
Katarzyna Haverkamp - 978-3-631-75689-8

Downloaded from PubFactory at 01/11/2019 02:56:45AM

via free access 


\section{Kapitel 6}

\section{Zusammenfassung und Ausblick}

\subsection{Zusammenfassung der Ergebnisse}

Die im Verlauf der Arbeit gewonnenen Ergebnisse wurden ausführlich in den abschließenden Abschnitten der jeweiligen Kapitel diskutiert. Daher soll nachfolgend auf eine vollständige Auflistung aller Teilergebnisse verzichtet werden. Stattdessen soll an dieser Stelle eine stichpunktartige Zusammenfassung der erfolgten Analyse, der grundlegenden Argumentationsbausteine und der gewonnenen Hauptergebnisse vorgenommen werden.

- Den Ausgangspunkt der vorliegenden Untersuchung bildete die Erkenntnis, dass FuE-Prozesse zunehmend arbeitsteilig verlaufen. Die von der Wissenschaftsstatistik erhobenen Daten zu FuE-Tätigkeit deutscher Unternehmen zeigen, dass im Zeitraum zwischen 1979 und 2003 der Anteil der für den externen Bezug von FuE-Dienstleistungen aufgewandten Mittel im Verhältnis zu den gesamten FuE-Aufwendungen der Unternehmen kontinuierlich von etwa $5 \%$ auf über $18 \%$ angestiegen ist. Internationale Daten bestätigen zudem den Bedeutungszugewinn der vertraglichen Kooperationen im FuE-Bereich. Während jedoch FuEKooperationsbeziehungen in der neueren innovationsökonomischen $\mathrm{Li}$ teratur verstärkt einer theoretischen und empirischen Analyse zugeführt werden, existieren bisher nur einige wenige Beiträge, die sich explizit der Untersuchung von Vertragsforschungsaktivitäten im Wirtschaftssektor widmen. 
- Das als empirisch bedeutsam eingestufte, jedoch bisher theoretisch wie empirisch wenig untersuchte Phänomen der Vertragsforschung sollte in der vorliegenden Arbeit einer systematischen wirtschaftswissenschaftlichen Analyse zugeführt werden. Das Ziel der Untersuchung war es, auf der Basis einer theoriegeleiteten Analyse Erkenntnisse bezüglich der Bestimmungsfaktoren der Vertragsforschung zu identifizieren und empirisch anhand von Daten zur Forschungstätigkeit deutscher Unternehmen zu überprüfen.

- Die Vertragsforschung wurde für die Zwecke der vorliegenden Arbeit als eine Vereinbarung zwischen Auftraggeber und Auftragnehmer definiert, bei der sich der Auftragnehmer (FuE-Dienstleister) verpflichtet, eine festgelegte Forschungs- und Entwicklungsaufgabe (FuE-Dienstleitung) durchzuführen und die Verfügungsrechte an dem gewonnen Wissen gegen Vergütung auf den Auftraggeber zu übertragen. Mit dieser Begriffsbestimmung wurde gewährleistet, dass Vertragsforschungsbeziehungen von Kooperationsbeziehungen abgegrenzt werden konnten und zur empirischen Erfassung des Phänomens der Vertragsvergabe die statistische Kategorie der "externen FuE-Aufwendungen“ herangezogen werden konnte. Forschungs- und Entwicklungsleistungen wurden dabei in Übereinstimmung mit den Vorgaben des Frascati-Handbuchs abgegrenzt.

- Es wurde auf die Besonderheit des kumulativen Charakters der FuE-Tätigkeit hingewiesen. Aus faktorkombinationsorientierter Betrachtungsweise kann FuE als ein Produktionsvorgang definiert werden, bei dem neben der kreativen menschlichen Arbeit auch das bereits vorhandene Wissen die zentralen Inputfaktoren darstellen. Auf der Outputseite stehen ihnen das immaterielle Ergebnis in der Form einer Konzeption einer technologischen Neuerung sowie im Zuge der FuE-Prozesse angesammelte Erfahrung und Know-how gegenüber. Wissen - mit den Eigenschaften einer frei diffundierenden "Information" sowie als personenbzw. organisationsgebundenes „Know-how" - stellt somit zugleich den zentralen Input- als auch Outputfaktor der FuE-Tätigkeit dar.

- Die Auseinandersetzung mit der neuesten empirischen Evidenz zu den allgemeinen Bestimmungsfaktoren der FuE-Tätigkeit im Wirtschaftssektor zeigte, dass von einer generellen technologischen Überlegenheit der in konzentrierten Märkten agierenden Großunternehmen im Sinne der beiden Neo-Schumpeter-Hypothesen nicht ausgegangen werden kann. Kleine und große Unternehmen weisen eher spezifische Vor- und Nachtei- 
le auf, die zur Ausdifferenzierung der jeweiligen Innovationsstrategien führen und eine komplementäre Arbeitsteilung im Technologiebereich nahe legen. Der komparative Vorteil von großen Unternehmen ist vorwiegend finanzieller Natur: Aufgrund des starken Innenfinanzierungspotenzials sind diese Unternehmen auch in der Lage, erhebliche Fixkosten der FuE-Tätigkeit zu tragen und können sich verstärkt im Bereich der Prozessentwicklung betätigen. Als umsatzstarke Wirtschaftseinheiten sind Großunternehmen in der Lage, die entstandenen Kosten auf eine größere Absatzmenge zu streuen. Kleine Unternehmen genießen dagegen Vorteile behavioraler Natur: leistungsfördernde, direkte Verbundenheit der Mitarbeiter mit den Forschungsprojekten und Unternehmenszielen, Fähigkeit zu schnellen Anpassungsreaktionen und Risikofreude. Dadurch können kleine Unternehmen flexibel auf die dem Wandel unterliegende Marktanforderungen reagieren und kundenspezifische Produkte und Technologien zügiger entwickeln.

- Im Zuge der Auseinandersetzung mit dem Einfluss der Marktstruktur auf die Entscheidungen der gewinngesteuerten Akteure im FuE-Bereich hat sich gezeigt, dass bei der Untersuchung von entscheidungsrelevanten Faktoren stärker auf Branchencharakteristika wie technologiefeldspezifisches Nachfragepotenzial und die Spillover-Problematik abgestellt werden sollte. So scheint die Erwartung einer positiven mittelfristigen Nachfrageentwicklung nicht nur Entscheidungen für die FuE-Tätigkeit zu begünstigen, sondern auch insbesondere einen starken Einfluss auf die Innovationsbereitschaft der Klein- und Mittelbetriebe auszuüben. Es wurde zudem betont, dass bei der Analyse der Innovationsentscheidungen der gewinngesteuerten Wirtschaftsakteure stets die Problematik der Externalitäten der FuE-Tätigkeit beachtet werden sollte. Die Schwierigkeit, sich den Nutzen aus eigener FuE-Tätigkeit vollständig anzueignen, kann die Entscheidungen für die Aufnahme bzw. Ausweitung privatwirtschaftlicher FuE negativ beeinflussen. Die Fähigkeit, von den im technologischen Umfeld eines Unternehmens vorliegenden externen Wissensressourcen zu profitieren, kann zur Senkung eigener Entwicklungskosten führen und folglich innovationsfördernd wirken.

- Im theoretischen Teil der Arbeit wurde das Phänomen der Vertragsforschungsaktivitäten aus der Perspektive von drei verschiedenen Forschungsrichtungen beleuchtet. Aus transaktionskostenorientierter Sichtweise können Organisationsentscheidungen der Wirtschaftsakteure vor dem Hintergrund der Transaktionskosteneinsparung analysiert wer- 
den. Der Property-Rights-Ansatz lenkt das Augenmerk auf die Rolle der Ressourcenverteilungsmuster zwischen den Wirtschaftsakteuren und ermöglicht damit die Untersuchung der Organisationsentscheidungen vor dem Hintergrund der unterschiedlichen Ressourcensituation der Unternehmen. Gemeinsam ist diesen Theorierichtungen, dass in der Analyse die Problematik der Unvollständigkeit von Verträgen und der resultierenden Hold-up-Gefahr ausdrücklich beachtet wird. Die industrieökonomischen Modelle der FuE-Kooperationen gestatten eine vertiefte Analyse des Einflusses von Externalitäten der FuE-Tätigkeit auf die Organisationsentscheidungen im FuE-Bereich.

- Insgesamt werden Vertragsforschungsbeziehungen vor dem Hintergrund der starken wechselseitigen Abhängigkeiten zwischen den Transaktionspartnern untersucht. Diese Abhängigkeiten entstehen zum einen aufgrund der Tatsache, dass eine vollständige Spezifizierung von FuEVertägen besonders schwierig ist. Die Unvollständigkeit der Verträge hat sodann die Entstehung von mehreren Gefahren der arbeitsteiligen FuEProzesse zur Folge. Erstens kann eine unter dem erwünschten Niveau liegende Investition der Vertragsparteien im Bereich der transaktionsspezifischen Investitionen nicht ausgeschlossen werden. Zweitens entsteht im Falle getätigter hochspezifischer Investitionen das Risiko eines Holdups durch eine Vertragspartei. Drittens kann der Auftraggeber nicht verifizieren, ob alle im Rahmen einer Vertragsbeziehung angesammelten innovationsrelevanten Erkenntnisse vom Auftragnehmer vollständig übertragen wurden und ob sie von der beauftragten Partei nicht in weiteren Vertragsforschungsbeziehungen mit anderen Partnern verwertet bzw. weitergegeben werden. Viertens führt die Notwendigkeit einer ständigen Interaktion in der Vertragsabwicklungsphase zur Entstehung der Gefahr einer unkontrollierten Wissensdiffusion zwischen den Transaktionspartnern. Die Existenz dieser Risiken wirkt hemmend auf die Bereitschaft der Wirtschaftsakteure, FuE-Dienstleistungen anzubieten bzw. nachzufragen. Mit dem Verweis auf diese Gefahren kann begründet werden, warum der internen Steuerung der FuE-Prozesse nach wie vor der Vorzug gegeben wird.

- Zum anderen befinden sich die Wirtschaftsakteure in der Situation einer wechselseitigen Interdependenz, da - wie in der Industrieökonomik hervorgehoben wird - der Nutzen aus eigener FuE-Tätigkeit nicht vollständig appropriierbar ist. Die Unternehmen berücksichtigen die Möglichkeit der Internalisierung von Externalitäten der 
FuE-Tätigkeit bei der Festlegung der eigenen FuE-Strategie. Eigene FuE-Entscheidungen werden damit unter Berücksichtigung der FuEEntscheidungen von Wettbewerbern getroffen.

- Den Gefahren der arbeitsteiligen Wissenserzeugung stehen jedoch einige Vorteile dieser Organisationsform gegenüber. So können durch den Rückgriff auf die im technologischen Umfeld eines Unternehmens bereits vorhandenen Wissensressourcen und spezifisches Know-how beträchtliche Zeitvorteile erzielt werden. Die externen Koordinationsformen der FuE-Prozesse bieten auch für Unternehmen der Spitzentechnologie die Möglichkeit, die im technologischen Umfeld eines Unternehmens stattfindenden neuen Entwicklungen zu verfolgen, ohne dass in einem unsicheren Umfeld unternehmensinterne FuE-Kapazitäten aufgebaut werden müssen. Auf diese Weise kann auch die Gefahr eines lockouts (Cohen/Levinthal (1990))- einer zu späten Anerkennung der Bedeutung eines dynamischen Technologiefeldes - vermieden werden. Vertragsforschung eröffnet auch Flexibilisierungspotenziale, wenn auf das Instrument der externen Wissensbeschaffung bei kurzfristig auftretenden Kapazitätsengpässen zurückgegriffen wird.

- Die Industrieökonomik macht zusätzlich auf einen besonderen Aspekt der externen Wissensbeschaffung aufmerksam. Es wird postuliert, dass die interne Absorptionsfähigkeit, aufgebaut durch die innerbetriebliche FuE-Tätigkeit, eine Voraussetzung für die Übernahme des unternehmensextern erarbeiteten Wissens darstellt. Damit wird anerkannt, dass das im technologischen Umfeld vorhandene Wissen nicht kostenfrei von jedem Unternehmen internalisiert werden kann, sondern nur von denjenigen Wirtschaftsakteuren, die eigene FuE-Kompetenzen aufweisen.

- Schließlich führt die theoretische Analyse zu der Schlussfolgerung, dass das Potenzial einer zur unternehmensinternen FuE-Tätigkeit substitutiven externen Wissensbeschaffung als eingeschränkt einzuschätzen ist. Unternehmen ohne eigene Innovationskompetenz sollte es zum einen schwer fallen, die im Rahmen eines FuE-Vertrages zu erbringende Forschungsleistungen $\mathrm{zu}$ spezifizieren und $\mathrm{zu}$ verifizieren. Zudem ist das Potenzial einer substitutiven Wissensbeschaffung angesichts der für die Übernahme und reibungslose Verwertung der zugekauften Forschungsergebnisse notwendigen Absorptionsfähigkeit eingeschränkt.

- Auf eine Wiederholung der Diskussion der empirischen Evidenz zu den einzelnen im Zuge der theoretischen Auseinandersetzung mit dem Phäno- 
men der Vertragsforschung hergeleiteten Hypothesen wird an dieser Stelle unter Verweis auf die Ausführungen des Abschnittes 5.5 verzichtet. Statt dessen sollen einige zentrale Schlussfolgerungen aus der vorgenommenen statistischen Analyse festgehalten werden:

- Insgesamt ist die Vertragsforschung besonders unter den großen, forschungsintensiven und in einem durch hohe Wettbewerbsintensität gekennzeichneten Umfeld agierenden Unternehmen ein weit verbreitetes Instrument, das zur Stärkung der eigenen FuE-Kompetenzen durch Integration unternehmensextern vorhandenen Know-hows herangezogen wird. Der Zugriff auf unternehmensextern vorhandene FuE-Kompetenzen und Ressourcen erweist sich als die zentrale Determinante der externen Wissensbeschaffung.

- Für die Übernahme unternehmensextern erarbeiteten Wissens ist die interne Absorptionsfähigkeit eine wichtige Voraussetzung: Von der Variable fuekon geht in jedem geschätzten Modell ein signifikant positiver Einfluss auf die Wahrscheinlichkeit der Vertragsvergabe aus.

- Die FuE-Dienstleister sind unter denjenigen Unternehmen zu suchen, die ihre eigene FuE-Kompetenz dank kontinuierlicher Auseinandersetzung mit technologischen Fragestellungen im Rahmen unternehmensinterner FuE-Tätigkeit aufgebaut haben und durch höhere Humankapitalintensität gekennzeichnet sind. Ein größenbedingter Vorteil der kleinen Betriebe im Bereich der Durchführung von FuE-Leistungen kann im Lichte der Ergebnisse der vorliegenden Arbeit nicht ausgeschlossen werden.

- Eine Gegenüberstellung der Ergebnisse zu den Bestimmungsfaktoren der Auftragsvergabe und $\mathrm{zu}$ den Determinanten der Durchführung von FuE-Aufträgen liefert einen Hinweis auf die vertiefte Arbeitsteilung zwischen den FuE-aktiven Unternehmen. Allerdings lassen die Ergebnisse der statistischen Analyse nicht darauf schließen, dass die wirtschaftsinternen Verflechtungen vorwiegend auf eine Arbeitsteilung zwischen den produzierenden Unternehmen und den "reinen Forschungsdienstleistern" zurückzuführen sind. Zu den Vertragsforschungsanbietern gehören nicht nur Unternehmen der wissensintensiven Branchen des Dienstleistungssektors (Datenverarbeitung und Datenbanken, Forschung und Entwicklung, Dienstleistungen für Unternehmen), sondern auch FuE-aktive Unternehmen des produzierenden Gewerbes. 
- Eine Erklärung dafür, dass die Angebotsseite des FuEDienstleistungsmarktes in Deutschland offensichtlich nicht von „reinen FuE-Dienstleistern" dominiert wird, kann darin gesucht werden, dass - wie die Ergebnisse der ifo-Studie zeigen - die fehlende Produktionserfahrung als eine bedeutsame Schwäche von "reinen Dienstleistern" aufgefasst wird. Nicht produzierende Unternehmen haben demnach einen komparativen Nachteil in Gestalt fehlender Erfahrung bei der Überführung prototypischer Entwicklungen in die Serienproduktion.

Die ökonometrische Analyse zeigte zudem, dass die Wahrscheinlichkeit der Durchführung von FuE-Leistungen mit der hohen Einschätzung der Kunden als Wissensquelle korreliert. Dies kann als eine Bestätigung der Hypothese von Pisano interpretiert werden, wonach die Neigung, FuE-Leistungen anzubieten, maßgeblich vom Streben der FuE-aktiven Unternehmen geleitet wird, den Zugang zu nachgelagerten Kompetenzen in der Produktion und Vermarktung fertiger innovativer Produkte zu erhalten.

- Ferner liefert die statistische Untersuchung einen Hinweis darauf, dass ein Teil der Vertragsforschungsbeziehungen neuerdings auf konzerninterne Verflechtungen zurückgeführt werden kann. Insbesondere zeigt sich in der ökonometrischen Schätzung ein Unterschied zwischen den Ergebnissen für das Jahr 1993 und das Jahr 2003. Von der Dummy-Variable, die die Konzernzugehörigkeit der Betriebe auffasst, geht in der Schätzung anhand von Daten des MIP 1993 kein signifikanter Einfluss auf die Wahrscheinlichkeit der Auftragsvergabe aus. Die Ergebnisse der Schätzungen anhand von Daten des MIP 2003 lassen dagegen auf einen signifikant positiven Einfluss der Konzernzugehörigkeit auf die Wahrscheinlichkeit der Auftragsvergabe schließen.

- Dem Motiv einer mit der Auftragsvergabe antizipierten Kostensenkung kommt im Lichte der Ergebnisse vorliegender Studien sowie der statistischen Untersuchung der vorliegenden Arbeit eine nur untergeordnete Rolle zu. Allerdings wird in der Literatur darauf hingewiesen, dass Rentabilitätsüberlegungen implizit in anderen der durch Unternehmen genannten Motiven der Auftragsvergabe enthalten sind. So kann im Sinne der innovationsökonomischen Literatur, die sich der Untersuchung des Einflusses der technologischen Möglichkeiten auf Entscheidungen im FuE-Bereich widmet, argu- 
mentiert werden, dass der Zugriff auf die im unternehmensexternen Umfeld vorhandenen Wissensressourcen nicht nur die Erzielung von Zeit-, sondern auch von Kostenvorteilen ermöglicht.

Die empirische Literatur zum deutschen FuE-Dienstleistungsmarkt formuliert die Einschätzung, dass von einer künftig weiter steigenden Rolle dieser Organisationsform der FuE-Tätigkeit im Wirtschaftssektor ausgegangen werden kann. Allerdings finden sich sowohl in der Literatur als auch unter den Ergebnissen der vorliegenden Arbeit Hinweise darauf, dass eine Veränderung der Innovationsstrategie derjenigen Unternehmen stattfindet, die ihren Wettbewerbsvorteil auf die FuE-Leistungen von Zulieferern stützen. Diese Unternehmen gehen dazu über, die extern erbrachten FuE-Dienstleistungen nicht direkt zu vergüten, sondern die FuE-Leistungen der Zulieferer bei der Preissetzung für die bezogenen innovativen Produkte zu berücksichtigen. Da die für den Bezug der innovativen Produkte getätigten Zahlungen nicht als externe FuE-Aufwendungen erfasst werden, kann diese Entwicklung zur Reduzierung der feststellbaren Transaktionsvolumina auf dem FuE-Dienstleistungsmarkt führen.

\subsection{Wirtschaftspolitische Implikationen}

Auf der Grundlage einer systematischen Analyse des Phänomens der arbeitsteiligen FuE-Prozesse im Wirtschaftssektor Deutschlands, wie sie in dieser Arbeit vorgenommen wurde, soll nachfolgend zunächst der Frage nach der wirtschaftspolitischen Beurteilung dieser Koordinationsform der Ressourcenallokation nachgegangen werden.

Aus der Sicht der Industrieökonomik kann von einer Vorteilhaftigkeit der horizontalen Zusammenarbeit von Wirtschaftsakteuren im FuE-Bereich ausgegangen werden, wenn das technologische Umfeld der Unternehmen von erheblichen Spilloverraten gekennzeichnet ist. Sind Vertragsforschungsbeziehungen jedoch als Transaktionen aufzufassen, die im Umfeld der Unvollständigkeit der Verträge von Partnern abgewickelt werden, die sich in vertikaler Beziehung zueinander befinden, dann kann auf die von der Theorie explizit modellierten Gefahren dieser Organisationsform hingewiesen werden. So gelingt es im Rahmen des Property-Rights-Ansatzes zu zeigen, dass es bei Vertragsbeziehungen zur Verzerrung der Investitionsbereitschaft kommen kann, d.h. zur Unterinvestition (underinvestment) im Bereich der transaktionsspezifischen Investitionen. Auch der Transaktionskostenansatz postuliert, dass auf 
eine marktliche Koordination der Ressourcenallokation primär bei geringerer Faktorspezifität zurückgegriffen wird. Es kann folglich postuliert werden, dass arbeitsteilige FuE-Prozesse zur Unterversorgung im Bereich der Erarbeitung hochspezifischer Wissensressourcen führen können.

Zu diesem Ergebnis kommen auch Kamien und Zang (2000), wenn sie im Rahmen industrieökonomischer Modelle der FuE-Kooperation dem Phänomen der Absorptionsfähigkeit explizit Rechnung tragen. Es zeigt sich dann, dass Unternehmen, die eine FuE-Kooperation mit einem Wettbewerber anstreben, eine FuE-Strategie wählen, die auf die Erarbeitung genereller - und nicht firmenspezifischer - Wissensfortschritte abzielt. Die breiter angelegte Forschungsstrategie ermöglicht es diesen Unternehmen, Absorptionsfähigkeit aufzubauen und von den FuE-Ergebnissen des Konkurrenten zu profitieren. Firmenspezifische Wissensressourcen werden nur dann aufgebaut - bzw. eine „spezifische FuEStrategie“ dann gewählt - wenn keine Zusammenarbeit mit Wettbewerbern angestrebt wird.

Im Ergebnis führt dies zu der Aussage, dass durch arbeitsteilige FuE-Prozesse erhebliche Zeitvorteile realisiert werden können, wenn einzelne Unternehmen auf die bereits im Umfeld vorhandenen FuE-Kompetenzen zugreifen können und die in der Innovationstätigkeit benötigten Teilergebnisse nicht erst intern erforschen müssen. Arbeitsteilige Wissensproduktion lässt jedoch zugleich die Gefahr entstehen, dass es zu - aus volkswirtschaftlicher Sicht - unerwünschter Unterversorgung mit der Produktion hochspezifischer Wissensressourcen kommt.

Die Anerkennung des Postulats, dass für die Übernahme der unternehmensextern erarbeiteten Wissensfortschritte die interne Absorptionsfähigkeit eine Voraussetzung darstellt, hat zugleich Konsequenzen für die Frage nach der Wirkungsrichtung von potenziellen Maßnahmen zur Förderung des Instruments der Vertragsforschung. Bereits die Evaluierungen der Fördermaßnahme „Externe Vertragsforschung“ haben gezeigt, dass ein externer Bezug von FuELeistungen von denjenigen Unternehmen in Anspruch genommen wurde, die eine vergleichsweise starke interne FuE-Kompetenz aufwiesen. Auch die Ergebnisse dieser Studie führen zu der Schlussfolgerung, dass von einer Komplementarität der externen Wissensbeschaffung und der internen Wissenserzeugung auszugehen ist. Die Förderung der Vertragsforschung kann folglich primär als eine Maßnahme zur Stärkung vorhandener Innovationskompetenz der ohnehin FuE-aktiven Unternehmen aufgefasst werden, bzw. als ein Instrument, mit dem eine intensivere - zum Teil branchenübergreifende - Verflechtung der FuE-aktiven Unternehmen erreicht werden kann. Die Wirksamkeit der Förde- 
rung der Vertragsforschung als Instrument, mit welchem der Aufbau interner FuE-Kapazitäten unterstützt wird, d.h. mit dem Innovationsvoraussetzungen geschaffen werden können, ist dagegen im Lichte der bisherigen Erkenntnisse nur als begrenzt einzuschätzen.

Schließlich ist darauf hinzuweisen, dass im Lichte der Ergebnisse der vorliegenden Arbeit die Finanzierungsstrategie der Fraunhofer-Gesellschaft (FhG)als einer Trägerorganisation von Einrichtungen der angewandten Forschung in Deutschland, die Vertragsforschungsdienstleistungen für Industrie- und Dienstleistungsunternehmen sowie die öffentliche Hand anbietet ${ }^{490}$ - positiv zu beurteilen ist. Im Leistungsbereich Vertragsforschung der FhG werden zwei Drittel der Einnahmen mit Aufträgen aus der Industrie und mit öffentlich finanzierten Forschungsprojekten erwirtschaftet; ein Drittel steuern der Bund und die Länder im Rahmen institutioneller Förderung bei. Die öffentliche Teilfinanzierung ermöglicht es den Instituten der Fraunhofer-Gesellschaft, Vorlaufforschung auf den jeweils relevanten Gebieten zu leisten. Vor dem Hintergrund der Erkenntnis der vorliegenden Arbeit, dass der Zugriff auf bereits vorhandene unternehmensexterne Ressourcen das zentrale Motiv der privatwirtschaftlichen Auftragsvergabe darstellt, ist die Erarbeitung der eigenen technologischen Vorlaufkompetenz eine Voraussetzung für die Attrahierung von FuE-Aufträgen. In diesem Sinne eröffnet die öffentliche Förderung der FhG die Möglichkeit, in Eigenforschung spezifische FuE-Kompetenzen auf ausgewählten Technologiegebieten aufzubauen und Wissensfortschritte zu erarbeiten, die für industrielle Nachfrager nach FuE-Leistungen relevant sein können.

\subsection{Implikationen für die Innovationsfor- schung}

In diesem Abschnitt sollen einige Implikationen der vorgenommenen Analyse für die weitere innovationsökonomische Forschung formuliert werden. Erstens kann darauf verwiesen werden, dass in der in dieser Arbeit ausgewerteten Literatur zunehmend anerkannt wird, dass das technologische Wissen aneigenbarer sein kann, als es in der klassischen Konzeptualisierung vom Wissen als öffentlichem Gut angenommen wird. Die erläuterten Ergebnisse der Untersuchungen zu Aneignungsmechanismen zeigen, dass Unternehmen mehrere Möglichkeiten haben, das unternehmensintern erarbeitete Wissen vor unkontrollierter Diffu-

490 Vgl. Fraunhofer-Gesellschaft (2005), S. 11. 
sion zu schützen, um den dank eigener FuE-Tätigkeit entstehenden Nutzen zu internalisieren. Nicht nur staatlich gewährten Schutzrechten kommt hierbei eine Rolle zu, sondern auch strategischen Mechanismen wie zeitlicher Vorsprung, Bindung qualifizierten Personals und Geheimhaltung. Die Anerkennung der Prämisse, dass nur ein Teil der Forschungsergebnisse unfreiwilliger Diffusion unterliegt und ein Teil des Wissen - vorwiegend impliziter Natur - geschützt werden kann vor der ungewollten Verbreitung und Anwendung durch Wettbewerber, führt zu der sich zunehmend durchsetzenden Einschätzung, dass „R\&D activity becomes more of a private good“491.

Zugleich liefert die Literatur auch einen Hinweis darauf, dass Spilloverraten branchencharakteristisch sein können: Während in der Pharma-Industrie von einem - komparativ gesehen - erheblichen Anteil des gut kodifizierbaren Wissens ausgegangen wird, kann vermutet werden, dass in Branchen wie der Maschinenbau oder die Messtechnik das Wissen prozessualer Natur eine große Rolle spielt. Für die weitere Forschung wäre die Erarbeitung einer Klassifikation der Wirtschaftszweige hilfreich, die es ermöglicht, zwischen Technologiegebieten zu unterscheiden, in welchen die Problematik der unvollständigen Appropriierung eine wichtige Rolle als Innovationshemmnis spielen kann, und Branchen, bei denen aufgrund einer besonderen Stellung der Wissenskomponenten mit Know-how-Charakter, die privatwirtschaftlichen FuE-Entscheidungen weniger stark von der Spilloverproblematik beeinflusst werden.

Einen Hinweis auf einen möglichen, in weiteren Erhebungen zu berücksichtigenden Aspekt liefern die Ergebnisse der Untersuchung über den Einfluss von technologischen Möglichkeiten. In den Schätzungen zeigte sich, dass die Wahrscheinlichkeit der Auftragsvergabe positiv von der höheren Einschätzung der Bedeutung wissenschaftlicher Wissensquellen und negativ von der hohen Einschätzung der Bedeutung des Wissens von Zulieferern beeinflusst wird. Der Einfluss der Variable, die die Bedeutung der Kunden und Wettbewerber auffasste, zeigte sich dagegen als durchgehend insignifikant. Zugleich weisen die Ergebnisse der Sonderauswertung der Wissenschaftsstatistik darauf hin, dass es sich bei den Vertragsforschungsbeziehungen mehrheitlich um wirtschaftsinterne Verflechtungen handelt und nicht um eine Zusammenarbeit zwischen der Wirtschaft und der Wissenschaft. An wen gehen dann die FuE-Verträge, wenn nicht an die Wissenschaft, Zulieferer oder Kunden?

Es kann vermutet werden, dass für die Gruppe der auftragserteilenden Unternehmen andere als die bisher in Erhebungen erfassten Partner relevant sind.

491 Cohen/Levinthal (1990), S. 142 
Insbesondere dürfte vermutet werden, dass Unternehmen anderer Branchen eine herausragende Rolle in der arbeitsteiligen Wissensproduktion spielen. Geht man davon aus, wie in der einschlägigen Literatur häufig postuliert wird, dass die Bedeutung der integrativen Technologien wächst, dann kann vermutet werden, dass Unternehmen verschiedener Forschungsfelder zunehmend auf gegenseitige Erfahrungen, Kenntnisse und FuE-Kompetenzen angewiesen sind. So argumentiert Hermes anhand eines Beispiels:

\begin{abstract}
„Zur Veranschaulichung dieser zunehmenden Vernetzung von Technologien sei der Werkzeugmaschinenbau angesprochen. Während noch vor 20 bis 30 Jahren ein Hersteller von Werkzeugmaschinen bei der Entwicklung neuer Produkte hauptsächlich auf dem Gebiet der mechanischen Konstruktion kompetent sein musste, hat ein moderner Werkzeugmaschinenbauer heute die Bereiche von mechanischer über elektrische Konstruktion und Mikroelektronik bis hin zur Softwareentwicklung abzudecken. “492
\end{abstract}

Können einzelne Unternehmen die Technologiebereiche nicht abdecken, welche für die Entwicklung eines auf integrativen Technologien beruhenden Produktes benötigt werden, so sind sie auf den Rückgriff auf unternehmensexterne Kompetenzen angewiesen. In diesem Falle findet jedoch weder ein Bezug einer FuE-Leistung innerhalb einer Branche (d.h. von einem Wettbewerber) noch ein Bezug der benötigten Entwicklung von einem Kunden statt, sondern eine Zusammenarbeit mit einem Unternehmen einer anderen Branche. Um für die Rolle der interindustriellen Verflechtungen im Technologiebereich kontrollieren zu können, müssen die einzelwirtschaftlichen Angaben zur Bedeutung der Unternehmen anderer Branchen als einer Wissensquelle gezielt erhoben werden.

Zur weiteren, vertieften Analyse des Phänomens der privatwirtschaftlichen Vertragsforschung bietet es sich schließlich an, folgenden Fragestellungen nachzugehen:

Erstens wäre es angebracht, explizit für Unterschiede bei den Motiven der Vertragsvergabe zwischen kleinen und großen Unternehmen zu kontrollieren. Hierfür könnten beispielweise getrennte Schätzungen für die unterschiedlichen Betriebsgrößenklassen durchgeführt werden.

Der Frage nach den Bestimmungsfaktoren der Vertragsvergabe in der Gruppe der kleinen ostdeutschen Unternehmen sollte insbesondere vor dem Hintergrund der Erkenntnis der vorliegenden Arbeit nachgegangen werden, wonach

492 Vgl. Hermes (1995), S. 1. 
kleine ostdeutsche Unternehmen häufiger zur Auftragsvergabe neigen als kleine westdeutsche Unternehmen. Eine gezielte Analyse könnte eine Antwort auf die Frage liefern, ob Erstere auf externe FuE-Leistungen verstärkt angesichts schwächerer interner FuE-Kompetenz zugreifen oder ob die feststellbaren Differenzen zwischen den west- und ostdeutschen Betrieben auf eine unterschiedliche Innovationsstrategie, Branchenstruktur und auf Unterschiede im Innovationsumfeld zurückzuführen sind.

Des Weiteren sollte untersucht werden, inwieweit eine verschiedenartige Motivation im Bereich der Beteiligung an Vertragsforschungsaktivitäten in Abhängigkeit vom Wirtschaftszweig der jeweiligen Unternehmen festgestellt werden können. Als Ausgangsbasis hierfür könnte die von Pavitt vorgeschlagene Branchentypologie verwendet werden.

Eine Herausforderung stellt schließlich eine Untersuchung des Zusammenhanges zwischen der Export- und FuE-Tätigkeit dar. In den empirischen Studien zu den allgemeinen Bestimmungsfaktoren der FuE-Tätigkeit zeigt sich vorwiegend ein signifikant positiver Zusammenhang. Die Ergebnisse der ökonometrischen Schätzungen anhand des Datensatzes der ersten Welle des MIP sowie die Studie über die Bestimmungsfaktoren der Auftragsvergabe in der Population der finnischen Unternehmen lassen zudem darauf schließen, dass die Wahrscheinlichkeit der Auftragsvergabe von der Exportintensität der Unternehmen positiv beeinflusst wird. Allerdings zeigt die Untersuchung anhand von Daten des MIP 2003, dass diese Ergebnisse nicht robust sind. Angebracht wären weitere Schätzungen mit alternativen Datensätzen bzw. eine gezielte Untersuchung des Zusammenhangs zwischen der Exporttätigkeit der Unternehmen und der durch diese Unternehmen verfolgten FuE-Strategie. 
Katarzyna Haverkamp - 978-3-631-75689-8

Downloaded from PubFactory at 01/11/2019 02:56:45AM

via free access 


\section{Literaturverzeichnis}

Acs, Z.J./Audretsch, D.B. (1990), Innovation and small firms, Cambridge, Mass.[u.a.]: MIT Press.

Adams, J.D./Chiang, E.P./Jensen, J.L. (2000), The influence of federal laboratory R\&D on industrial research, in: Review of Economics and Statistics, 85(4), S. 1003-1020.

Adams, J.D./Marcu, M. (2004), R\&D sourcing, joint ventures and innovation: a multiple indicators approach, NBER Working Paper No. 10474.

Aghion, Ph./Tirole, J. (1994), The management of innovation, in: Quarterly Journal of Economics, 109(4), S. 1185-1210.

Akerlof, G.A. (1970), The market for „lemons": quality uncertainty and the market mechanism, in: Quarterly Journal of Economics, 84(3), S. 488-500.

Alchian, A.A./Woodward, S. (1988), The firm is dead, long live the firm: a review of Oliver E. Williamson's The economic institutions of capitalism, in: Journal of Economic Literature, 26(1), S. 65-79.

Allesch, J./Fiedler, H./Martin, H.-J. (1983), Förderung der Auftragsforschung: Zwischenbilanz der Fördermaßnahme „Externe Vertragsforschung", Köln: Verlag TÜV Rheinland.

Amir, R. (2000), Modelling imperfectly appropriable R\&D via spillovers, in: International Journal of Industrial Organization, 18(7), S. 1013-1032.

Archibugi, D./Pianta, M. (1996), Innovation surveys and patents as technology indicators: the state of the art, in: OECD (Hrsg.), Innovation, patents and technological strategies, Paris: OECD, S. 17-56. 
Arora, A. (1995), Appropriating rents form innovation: a historical look at the chemical industry, in: Albach, A./Rosenkranz, S. (Hrsg.), Intellectual property rights and global competition: towards a new synthesis, Berlin: Ed. Sigma, S. 161-188.

Arora, A. (1997), Patents, licensing, and market structure in the chemical industry, in: Research Policy, 26(4), S. 391-403.

Arrow, K.J. (1962), Economic welfare and the allocation of resources for invention, in: NBER (Hrsg.), The rate and direction of inventive activity: economic and social factors; a conference of the Universities-National Bureau Committee for Economic Research and the Committee on Economic Growth of the Social Science Research Council, Princeton, NJ: Princeton Univ. Press, S. 609-625.

Arrow, K.J. (1969), The organization of economic activity: issues pertinent to the choice of market versus nonmarket allocation, in: The analysis and evaluation of public expenditures: the PBB system, Vol. 1, U.S. Joint Economic Committee, 91st Congress, 1st Session, Washington, DC: U.S. Government Printing Office, S. 59-73.

Arundel, A. (2001), The relative effectiveness of patents and secrecy for appropriation, in: Research Policy, 30(4), S. 611-624.

Arundel, A./Kabla, I. (1998), What percentage of innovations are patented?: empirical estimates for European firms, in: Research Policy, 27(2), S. 127-141.

Arvanitis, S. (1997), The impact of firm size on innovative activity - an empirical analysis based on Swiss firm data, in: Small Business Economics, $9(6)$, S. $473-490$.

Arvanitis, S./Hollenstein, H. (1994a), Demand and supply factors in explaining the innovative activity of Swiss manufacturing firms: an analysis based on input-, ouput- and market-oriented innovation indicators, in: Economics of Innovation and New Technology, 3(1), S. 15-30.

Arvanitis, S./Hollenstein, H. (1994b), Die Messung der Innovationsintensität: Eine empirische Untersuchung anhand schweizerischer Unternehmensdaten, in: Schweizerische Zeitschrift für Volkswirtschaft und Statistik, 130(3), S. 523-537. 
Arvanitis, S./Hollenstein, H. (1996), Industrial innovation in Switzerland: a model-based analysis with survey data, in: Kleinknecht, A.H. (Hrsg.), Determinants of innovation: the message from new indicators, Basingstoke [u.a.]: Macmillan, S. 13-62.

Arvanitis, S./Hollenstein, H. (2004), Firm panel data from the Swiss innovation survey, in: Schmollers Jahrbuch: Zeitschrift für Wirtschafts- und Sozialwissenschaften, 124(2), S. 305-314.

Arvanitis, S./von Arx, J. (2004), Bestimmungsfaktoren der Innovationstätigkeit und deren Einfluss auf Arbeitsproduktivität, Beschäftigung und Qualifikationsstruktur: eine mikroökonometische Untersuchung anhand von Paneldaten 1988-2002, Arbeitspapiere KOF/ETH Nr. 91.

Audretsch, D.B. (1995), Intellectual property rights: new research directions, in: Albach, A./ Rosenkranz, S. (Hrsg.), Intellectual property rights and global competition: towards a new synthesis, Berlin: Ed. Sigma, S. 35-76.

Azoulay, P. (2003), Acquiring knowledge within and across firm boundaries: evidence from clinical development, NBER Working Paper No. 10083.

Baldwin, W.L./Scott, J.T. (1987), Market structure and technological change, Chur [u.a.]: Harwood.

Becker, W./Dietz, J. (2002), R\&D cooperation and innovation activities of firms - evidence for the German manufacturing industry, Institut für Volkswirtschaftslehre der Universität Augsburg, Volkswirtschaftliche Diskussionsreihe Nr. 222.

Becker, W./Peters, J. (2005), Innovation effects of science-related technological opportunities: theoretical considerations and empirical findings for firms in the German manufacturing industry, in: Jahrbücher für Nationalökonomie und Statistik, 225(2), S. 130-149.

Beneito, P. (2003), Choosing among alternative technological strategies: an empirical analysis of formal sources of innovation, in: Research Policy, 32(4), S. 693-713.

Beneito, P. (2006), The innovative performance of in-house and contracted R\&D in terms of patents and utility models, in: Research Policy, 35(4), S. 502-517. 
Bester, H. (2004), Theorie der Industrieökonomik, 3., verb. Aufl., Berlin [u.a.]: Springer.

Björn, W.M. (2002), Messung und Bewertung der Leistung von Forschung und Entwicklung im Innovationsprozeß: Methodenüberblick, Entwicklung und Anwendung eines neuen Konzepts, Dissertation, Technische Universität Darmstadt.

Blind, K./ Edler, J./ Frietsch, R./ Schmoch, U. (2003), Erfindungen kontra Patente: Schwerpunktstudie „Zur technologischen Leistungsfähigkeit Deutschlands": Endbericht, Karlsruhe: ISI.

Bosch, F.A.J. van den/Elfferich, P.C. (1991), Make, buy or cooperate decisions with respect to the acquisition and exploitation of technical knowledge and organizational issues, Rotterdam: Rotterdam School of Management.

Braga, H.C./Willmore, L. (1991), Technological imports and technological effort: an analysis of their determinants in Brazilian firms, In: Journal of Industrial Economics, 39(4), S. 421-432.

Brockhoff, K. (1999), Forschung und Entwicklung: Planung und Kontrolle, 5., erg. und erw. Aufl., München [u.a.]: Oldenbourg.

Brouwer, E./Kleinknecht, A.H. (1996), Determinants of innovation: a microeconometric analysis of three alternative innovation output indicators, in: Kleinknecht, A.H. (Hrsg.), Determinants of innovation: the message from new indicators, Basingstoke [u.a.]: Macmillan, S. 99-124.

Brouwer, E./Kleinknecht, A.H. (1999a), Innovative output, and a firm's propensity to patent: an exploration of CIS micro data, in: Research Policy, 28(6), S. 615-624.

Brouwer, E./Kleinknecht, A.H. (1999b), Keynes-plus?: Effective demand and changes in firm-level $\mathrm{R} \& \mathrm{D}$; an empirical note, in: Cambridge Journal of Economics, 23(3), S. 385-391.

Bühler, S./Jaeger, F. (2002), Einführung in die Industrieökonomik, Berlin [u.a.]: Springer.

Bundesminister für Forschung und Technologie (1982), Die Messung wissenschaftlicher und technischer Tätigkeiten: allgemeine Richtlinien für 
statistische Übersichten in Forschung und experimenteller Entwicklung; Frascati-Handbuch 1980, Bonn.

Bundesministerium für Bildung und Forschung (2006), Bericht zur technologischen Leistungsfähigkeit Deutschlands 2006: Zentrale Aussagen der Bundesregierung, www. technologische-leistungsfähigkeit.de/ pub/tlf_2006_aussagen_breg.pdf, zugegriffen am 28.09.06.

Chatterji, D. (1996), Accessing external sources of technology, in: Research Technology Management, 39(2), S. 48-56.

Chiu, Y.S. (1998), Noncooperative bargaining, hostages, and optimal asset ownership, in: American Economic Review, 88(4), S. 882-901.

Chung, W.H. (1998), Spezifität und Unternehmungskooperation: Eine institutionenökonomische Analyse unter Berücksichtigung dynamischer Aspekte, Berlin: Duncker \& Humblot.

Coase, R.H. (1937), The nature of the firm. in: Economica, 4, S. 386-405.

Coase, R.H. (1960), The problem of social cost, in: Journal of Law \& Economics, 3, S. 1-44.

Cohen, W.M. (1995), Empirical studies of innovative activity, in: Stoneman, P. (Hrsg.), Handbook of the economics of innovation and technological change, Oxford [u.a.]: Blackwell, S. 182-264.

Cohen,W.M./Goto,A./Nagata,A./Nelson,R.R./Walsh,J.P. (2002), $\mathrm{R} \& \mathrm{D}$ spillovers, patents and the incentives to innovate in Japan and the United States, in: Research Policy, 31(8), S. 1349-1367.

Cohen, W.M./Klepper, S. (1992), The anatomy of industry R\&D intensity distributions, in: American Economic Review, 82(4), S. 773-799.

Cohen, W.M./Klepper, S. (1996a), A reprise of size and R\&D, in: Economic Journal, 106(437), S. 925-951.

Cohen, W.M./Klepper, S. (1996b), Firm size and the nature of innovation within industries: the case of process and product $R \& D$, in: Review of Economics and Statistics, 78(2), S. 232-243.

Cohen, W.M./Levin, R.C. (1989), Empirical studies of innovation and market structure, in: Schmalensee, R./Willig R.D. (Hrsg.), Handbook 
of industrial innovation, Vol. II, Amsterdam [u.a.]: North-Holland, S. 1059-1107.

Cohen, W.M./Levinthal, D.A. (1990), Absorptive capacity: a new perspective on learning and innovation, in: Administrative Science Quarterly, 35, S. 128-152.

Coombs, R./ Narandren, P./ Richards, A. (1996), A literature-based innovation output indicator, in: Research Policy, 25(3), S. 403-413.

Corsten, H. (1998), Produktionswirtschaft: Einführung in das industrielle Produktionsmanagement, 7., vollst. überarb. u. wesentl. erw. Aufl., München [u.a.]: Oldenbourg.

Crepon, B./Duguet, E. (1996), Innovation: measurement, returns and competition, in: INSEE Studies in Economics and Statistics, 1, S. 8295.

Crepon, B./Duguet, E./Kabla, I. (1996), Schumpeterian conjectures: a moderate support from various innovation measures, in: Kleinknecht, A.H. (Hrsg.), Determinants of innovation: the message from new indicators, Basingstoke [u.a.]: Macmillan, S. 63-98.

Croisier, B. (1998), The governance of external research: empirical test of some transaction-cost related factors, in: R\&D Management, 28(4), S. 289-298.

Czarnitzky, D. (2002), Research and Development: financial constraints and the role of public funding for small and medium-sized enterprises, ZEW Discussion Paper No. 02-74.

Czarnitzki, D. (2004), Das Innovationsverhalten von Unternehmen und die Rolle der Forschungs- und Technologiepolitik: ein Vergleich zwischen Ostund Westdeutschland, Dissertation, Universität Duisburg-Essen.

Dasgupta, P./Stiglitz, J. (1980), Industrial structure and the nature of innovative activity, in: Economic Journal, 90(358), S. 266-293.

D'Aspremont, C./Jacquemin, A. (1988), Cooperative and noncooperative R\&D in duopoly with spillovers, in: American Economic Review, 78(5), S. 1133-1137. 
D'Aspremont, C./Jacquemin, A. (1990), Cooperative and noncooperative R\&D in duopoly with spillovers: erratum, in: American Economic Review, 80(3), S. 641-642.

Demsetz, H. (1988), The theory of the firm revisited, in: Journal of Law, Economics, and Organization, 4(1), S. 141-161.

Dijk, B. van / den Hertog, R. / Menkveld, B. / Thurik, R. (1997), Some new evidence on the determinants of large- and small-firm innovation, in: Small Business Economics, 9(4), S. 335-343.

Dilling, A.A. (2002), Anwendung und Anwendbarkeit der Realoptionstheorie zur Bewertung von Forschungs- und Entwicklungsprojekten unter besonderer Berücksichtigung projektendogener Risiken, Göttingen: Sierke.

Dillmann, L. (1996), Outsourcing in der Produktentwicklung: Eine transaktionskostentheoretische Betrachtung der zunehmenden Fremdvergabe pharmazeutischer Produktentwicklungsaufgaben in der BRD, Frankfurt a. Main: Lang.

Dillmann, L. (1997), Die zunehmende Fremdvergabe pharmazeutischer Produktentwicklungsaufgaben an Contract Research Organisations Eine transaktionskostentheoretische Untersuchung, in: Schmalenbachs Zeitschrift für betriebswirtschaftliche Forschung, 49(12), S. 1047-1065.

Dohrmann, R.J. (1982), Quantifying contract research projects, in: Research Management, 25, S. 30-33.

Dubbermann, G. (1999), (Grundlagen-) Forschung, Externalitäten und „Marktversagen", in: List-Forum für Wirtschafts- und Finanzpolitik, 25(3), S. 271-288.

Duguet, E./Kabla, I. (2000), Appropriation strategy and the motivations to use the patent system; an economentric analysis at the firm level in French manufacturing, in: Encaoua, D./Hall, B.H./Laisney, F./Mairesse, J. (Hrsg.), The economics and econometrics of innovation, Boston, Mass. [u.a.]: Kluwer Acad. Publ., S. 267-305.

Dunning, J.H. (1997), Alliance capitalism and global business, London [u.a.]: Routledge.

Edlin, A.S./Reichelstein, S. (1996), Holdups, standard breach remedies, and optimal investement, in: American Economic Review, 86(3), S. 478501. 
Ehlken, J. (1999), Modelleinsatz bei der Bewertung und Auswahl von Forschungs- und Entwicklungsprojekten: Darstellung der Modelle, Analyse von Problemen und Identifikation von Lösungsansätzen, Göttingen [u.a.]: Hainholz-Verlag.

Eichin, K.-H. (1995), Internationale Perspektiven der Pharma-Industrie, in: Herzog, R. (Hrsg.), F\&E-Management in der Pharma-Industrie, Aulendorf: Editio-Cantor-Verlag, S. 54-79.

Englander, E.J. (1988), Technology and Oliver Williamsons's transaction cost economics, in: Journal of Economic Behavior \& Organization, 10(3), S. 339-353.

Erlei, M./Leschke, M./Sauerland, D. (1999), Neue Institutionenökonomik, Stuttgart: Schäffer-Poeschel.

Evangelista, R./Perani, G./Rapiti, F./Archibugi, D. (1997), Nature and impact of innovation in manufacturing industry: some evidence from the Italian innovation survey, in: Research Policy, 26(4-5), S. 521-536.

Feenstra, R.C. (1998), Integration of trade and disintegration of production in the global economy, in: Journal of Economic Perspectives, 12(4), S. $31-50$.

Felder, J./Licht, G./Nerlinger, E./Stahl, H. (1996), Factors determining $R \& D$ and innovation expenditure in German manufacturing industries, in: Kleinknecht, A.H. (Hrsg.), Determinants of innovation: the message from new indicators, Basingstoke [u.a.]: Macmillan, S. 125-154.

Foray, D. (1991), The secrets of industry are in the air: industrial cooperation and the organizational dynamics of innovative firm, in: Research Policy, 20(5), S. 393-405.

Foray, D. (1995), Knowledge distribution and the institutional infrastructure: the role of intellectual property rights, in: Albach, A./Rosenkranz, S. (Hrsg.), Intellectual property rights and global competition: towards a new synthesis, Berlin: Ed. Sigma, S. 77-117.

Fraunhofer ISI/Prognos AG (Hrsg.) (1991), FuE-Kooperationen von kleinen und mittleren Unternehmen: Evaluation der BMFT-Maßnahmen auf diesem Gebiet, Untersuchung im Auftrag des Bundesministeriums für Forschung und Technologie, Basel/Karlsruhe. 
Fraunhofer-Gesellschaft (2005), Jahresbericht 2005, www.fraunhofer.de/ fhg/Images/FhG-JB-komplett_tcm5-63602.pdf, zugegriffen am 21.09.06.

Frisch, A.J. (1993), Unternehmensgrösse und Innovation: die schumpeterianische Diskussion und ihre Alternativen, Frankfurt [u.a.]: CampusVerl.

Fritsch, M. (1993), Markt, Marktversagen und die Evaluation technologiepolitischer Förderprogramme, Faculty of Economics and Business Administration, Technical University Bergakademie Freiberg, Discussion Paper Nr. 93/8.

Fritsch, M./Wein, Th./Ewers, H.-J. (1996), Marktversagen und Wirtschaftspolitik: mikroökonomische Grundlagen staatlichen Handelns, 2., überarb. und erg. Aufl., München: Vahlen.

Gad, S.C. (2003), The selection and use of contract research organisations: a guide for the pharmaceutical and medical device industries, London [u.a.]: Taylor \& Francis.

Gauglitz-Lüter, S. (1998), Effektivitäts- und effizienzorientiertes Forschungs- und Entwicklungsmanagement, Lohmar [u.a.]: Eul.

Geroski, P.A./Walters, C.F. (1995), Innovative activity over the business cycle, in: Economic Journal, 105(431), S. 916-928.

Ghoshal, S./Moran, P. (1996), Bad for practice: a critique of the transaction cost theory, in: Academy of Management Review, 21(1), S. 13-47.

Gibbons, R. (2005), Four formal(izable) theories of the firm?, in: Journal of Economic Behavior \& Organization, 58(2), S. 200-245.

Goel, R.K./Rich, D.P. (2005), Organization of markets for science and technology, in: Journal of Institutional and Theoretical Economics, 161(1), S. 1-17.

Gottschalk, S./Janz, N. (2001), Innovation dynamics and endogenous market structure: econometric results from aggregated survey data, ZEW Discussion Paper No. 01-39.

Gottschalk, S./Janz, N. (2003), Bestimmungsfaktoren der Innovationstätigkeit, in: Janz, N./Licht, G. (Hrsg.), Innovationsforschung heute: die Mannheimer Innovationspanels, Baden-Baden: Nomos-Verl.-Ges., S. 17-39. 
Granstrand, O. / Bohlin, E. / Oskarsson, Ch. / Sjöberg, N. (1992), External technology acquisition in large multi-technology corporations, in: R\&D Management, 22(2), S. 111-133.

Grenzmann, Ch. (2004), Forschung und Entwicklung in der Wirtschaft: Die FuE-Statistik des Wirtschaftssektors, in: Legler, H./Grenzmann, Ch. (Hrsg.), Forschung- und Entwicklung in der deutschen Wirtschaft: Statistik und Analysen, Essen: Wissenschaftsstatistik, S. 7-17.

Griliches, Z. (1990), Patent statistics as economic indicators: a survey, in: Journal of Economic Literature, 28(4), S. 1661-1707.

Grossman, S.J./Hart O.D. (1986), The costs and benefits of ownership: a theory of vertical and lateral integration, in: Journal of Political Economy, 94(4), S. 691-719.

Grossman, G.M./Helpman, E. (1999), Incomplete contracts and industrial organization, NBER Working Paper No. 7303.

Grossman, G.M./Helpman, E. (2005), Outsourcing in a global economy, in: Review of Economic Studies, 72(250), S. 135-160.

Hagedoorn, J. (1995), Strategic technology partnering during the 1980s: trends, networks and corporate patterns in non-core technologies, in: Research Policy, 24(2), S. 207-231.

Hagedoorn, J. (2002), Inter-firm R\&D partnerships: an overview of major trends and patterns since 1960, in: Research Policy, 31(4), S. 477-492.

Hagedoorn, J./Cloodt, M. (2003), Measuring innovative performance: is there an advantage in using multiple indicators?, in: Research Policy, 32(8), S. 1365-1379.

Hagedoorn, J./ Link, A.N./ Vonortas, N.S. (2000), Research partnerships, in: Research Policy, 29(4-5), S. 567-586.

Haid, A./Weigand, J. (2001), R\&D, liquidity constraints, and corporate governance, in: Jahrbücher für Nationalökonomie und Statistik, 221(2), S. $145-167$.

Hall, B.H. (2002), The financing of research and development, in: Oxford Review of Economic Policy, 18(1), S. 35-51. 
Hansen, H. (1999), Die wettbewerbspolitische Beurteilung horizontaler Forschungs- und Entwicklungskooperationen, Berlin: Duncker \& Humblot.

Harabi, N. (1995), Appropriability of technical innovations: an empirical analysis, in: Research Policy, 24(6), S. 981-992.

Harhoff, D./Licht, G. (1996), Innovationsaktivitäten kleiner und mittlerer Unternehmen: Ergebnisse des Mannheimer Innovationspanels, BadenBaden: Nomos Verl.-Ges.

Hart, O.D. (1989), An economist's perspective on the theory of the firm, in: Columbia Law Review, 89, S. 1757-1774.

Hart, O.D. (1995), Firms, contracts and financial structure, Oxford [u.a.]: Clarendon Press.

Hart, O.D./Moore, J. (1990), Property rights and the nature of the firm, in: Journal of Political Economy, 98(6), S. 1119-1158.

Hart, O.D./Moore, J. (1999), Foundations of incomplete contracts, in: Review of Economic Studies, 66(226), S. 115-138.

Haour, G. (1992), Stretching the knowledge-base of the enterprise through contract research, in: R\&D Management, 22(2), S. 177-182.

Haug, H.-F./Revermann, Ch. (2003), Statistik für Forschung und experimentelle Entwicklung im Vergleich, Wirtschaft und Statistik, 12/2003, S. 1130-1136.

Hayek, F.A. (1945), The use of knowledge in society, in: American Economic Review, 35(4), S. 519-530.

Henriques, I. (1990), Cooperative and noncooperative R\&D in duopoly with spillovers: comment, in: American Economic Review, 80(3), S. 638640 .

Hermes, M. (1995), Eigenerstellung oder Fremdbezug neuer Technologie, Dissertation, Christian-Albrechts-Universität zu Kiel.

Hertog, R.G.J. den/Thurik, A.R. (1993), Determinants of internal and external R\&D: some Dutch evidence, in: De Economist, 141(2), S. 279289. 
Himmelberg, Ch.P./Petersen, B.C. (1994), R\&D and internal finance: a panel study of small firms in high-tech industries, in: Review of Economics and Statistics, 76(1), S. 38-51.

Hinloopen, J. (1997), Subsidizing cooperative and noncooperative R\&D in duopoly with spillovers, in: Journal of Economics, 66(2), S. 151-176.

Hinloopen, J. (2003), R\&D efficiency gains due to cooperation, in: Journal of Economics, 80(2), S. 107-125.

Hipp, Ch. (2000), Innovationsprozesse im Dienstleistungssektor: Eine theoretisch und empirisch basierte Innovationstypologie, Heidelberg: PhysicaVerlag.

Hippel, E. von (1987), Cooperation between rivals: informal know-how trading, in: Research Policy, 16(6), S. 291-302.

Hollenstein, H. (1996), A composite indicator of a firms's innovativeness: an empirical analysis based on survey data for Swiss manufacturing, in: Research Policy, 25(4), S. 633-645.

Hosmer, D.W./ Lemeshow, S. (1989), Applied logistic regression, New York, NY [u.a.]: Wiley.

Howells, J. (1997), Research and technology outsourcing, CRIC Discussion Paper No. 6.

Jacobsson, S./Oskarsson, Ch./Philipson, J. (1996), Indicators of technological activities - comparing educational, patent and R\&D statistics in the case of Sweden, in: Research Policy, 25(4), S. 573-585.

Jaffe, A.B. (1986), Technological opportunity and spillovers of R\&D: evidence from firms' patents, profits, and market value, in: American Economic Review, 76(5), S. 984-1001.

Jankowski, J.E. (2001), Measurement and growth of R\&D within the service economy, in: Journal of Technology Transfer, 26(4), S. 323-336.

Janz, N. /Ebling, G. /Gottschalk, S. /Niggemann, H. (2001), The Mannheim innovation panels (MIP and MIP-S) of the Centre for European Economic Research (ZEW), in: Schmollers Jahrbuch, 121(1), S. $123-129$. 
Janz, N./Ebling, G./Gottschalk, S./Peters, B. (2002), Die Mannheimer Innovationspanels, in: Allgemeines Statistisches Archiv, 86(2), S. 189-201.

Janz, N./Ebling, G./Gottschalk, S./Peters, B./Schmidt, T. (2003), Die Mannheimer Innovationspanels: Datenerhebung und -nutzung, in: Janz, N./Licht, G. (Hrsg.), Innovationsforschung heute: die Mannheimer Innovationspanels, Baden-Baden: Nomos-Verl.-Ges, S. 9-16.

Johnson, R.A./Wichern, D.W. (1999), Applied multivariate statistical analysis, Upper Saddle River, NJ [u.a.]: Prentice Hall.

Kaas, K.P./Fischer, M. (1993), Der Transaktionskostenansatz, in: Das Wirtschaftsstudium, 8-9, S. 686-693.

Kaltwasser, A. (1994), Wissenserwerb für Forschung \& Entwicklung: eine Make-or-buy-Entscheidung, Wiesbaden: Dt. Univ.-Verl. [u.a.].

Kamien, M.I./Muller, E./Zang, I. (1992), Research joint ventures and R\&D cartels, in: American Economic Review, 82(5), S. 1293-1306.

Kamien, M.I./Schwartz, N.L. (1975), Market structure and innovation: a survey, in: Journal of Economic Literature, 13(1), S. 1-37.

Kamien, M.I./Schwartz, N.L. (1978), Self-financing of an R\&D project, in: American Economic Review, 68(3), S. 252-261.

Kamien, M.I./Schwartz, N.L. (1982), Market structure and innovation, Cambridge [u.a.]: Cambridge Univ. Press.

Kamien, M.I./Zang, I. (2000), Meet me halfway: research joint ventures and absorptive capacity, in: International Journal of Industrial Organization, 18(7), S. 995-1012.

Kern, W./Schröder, H.-H. (1977), Forschung und Entwicklung in der Unternehmung, Reinbeck bei Hamburg: Rowohlt.

Keßler, U. (1992), Unternehmensgröße, Innovation und Wertschöpfungswachstum: Eine empirische Untersuchung im Lichte der Schumpeterschen Innovationsdiskussion, Frankfurt am Main [u.a.]: Lang.

KfW Bankengruppe (Hrsg.) (2005), KfW - Mittelstandspanel 2005, Frankfurt am Main. 
Kinkel, S./Lay, G. (2003), Fertigungstiefe - Balast oder Kapital?: Stand und Effekte von Out- und Insourcing im Verarbeitenden Gewerbe in Deutschland, Mitteilungen aus der Produktionsinnovationserhebung Nr. 30 .

Kiwit, D. (1994), Zur Leistungsfähigkeit neoklassisch orientierter Transaktionskostenansätze, in: Ordo, 45, S. 105-135.

Klein, B./Crawford, R.G./Alchian, A.A. (1978), Vertical integration, appropriable rents, and the competitive contracting process, in: Journal of Law \& Economics, 21(2), S. 297-326.

Kline, S.J./Rosenberg, N. (1986), An overview of innovation, in: Landau, R./Rosenberg, N. (Hrsg.), The positive sum strategy - harnessing technology for economic growth, Washington DC: National Academy Press.

Kleinknecht, A.H. (1987), Measuring R\&D in small firms: how much are we missing?, in: Journal of Industrial Economics, 36(2),] S. 253-256.

Kleinknecht, A.H. (1996), New indicators and determinants of innovation: an introduction, in: Kleinknecht, A.H. (Hrsg.), Determinants of innovation: the message from new indicators, Basingstoke [u.a.]: Macmillan, S. $1-11$.

Kleinknecht, A.H./van Montfort, K./Brouwer, E. (2000), How consistent are innovation indicators? A factor analysis of CIS data, Reserach Memorandum 2000-28.

Kleinknecht, A.H./Oostendorp, R. (2002), R\&D and export performance: taking account of simultaneity, in: Kleinknecht, A.H./Mohnen, P.A. (Hrsg.), Innovation and firm performance: econometric explorations of survey data, Basingstoke, Hampshire [u.a.]: Palgrave, S. 310-320.

Kleinknecht, A.H./Reijnen, J.O.N. (1991), More evidence on the undercounting of small firm R\&D, in: Research Policy, 20(6), S. 579-587.

Kleinknecht, A.H./Verspagen, B. (1988), Demand and innovation; a reexamination of the Schmookler hypothesis, Research memorandum 1988003.

Klodt, H. (1995), Grundlagen der Forschungs- und Technologiepolitik, München: Vahlen. 
Kogut, B./Zander U. (1992), Knowledge of the firm, combinative capabilities, and the replication of technology, in: Organization Science, 3, S. 383-397.

Kohler, U./Kreuter, F. (2001), Datenanalyse mit Stata: allgemeine Konzepte der Datenanalyse und ihre praktische Anwendung, München[u.a.]: Oldenbourg.

Kommission der Europäischen Gemeinschaften (1973), Die industrielle Auftragsforschung und -entwicklung in den sechs Ländern der Europäischen Gemeinschaften, Luxemburg: Europäische Gemeinschaften.

König, H./Licht, G. (1995), Patents, R\&D and innovation: evidence from the Mannheim innovation panel, in: ifo Studien, 41(4), S. 521-543.

Kortum, S./Lerner, J. (1999), What is behind the recent surge in patenting?, in: Research Policy, 28(1), S. 1-22.

Koschatzky, K./Reinhard, M./Grenzmann, Ch. (2003), Forschungsund Entwicklungsdienstleistungen in Deutschland: Struktur und Perspektiven eines Wachstumsmarktes, Stuttgart: Fraunhofer IRB Verlag.

Krahnen, J.P. (1991), Sunk costs und Unternehmensfinanzierung, Wiesbaden: Gabler.

Kreuzer, S. (1986), Forschungs- und Technologiepolitik zwischen Marktversagen und Staatsversagen, in: Kuhn, H. (Hrsg.), Probleme der Stabilitätspolitik: Festgabe zum 60. Geburtstag von Norbert Kloten, Göttingen: Vandenhoeck and Ruprecht, S. 87-105.

Kulicke, M./Broß, U./Gundrum, U. (1997), Innovationsdarlehen als Instrument zur Förderung kleiner und mittlerer Unternehmen: Evaluierung des FuE-Darlehenprogramms für kleine Unternehmen zur Anwendung neuer Technologien und des KfW-Innovationsprogramms, Heidelberg: Physica-Verl.

Kumar, N./Saqib, M. (1996), Firm size, opportunities for adaptation and in-house R\&D activity in developing countries: the case of Indian manufacturing, in: Research Policy, 25(5), S. 713-722.

Kurokawa, S. (1997), Make-or-buy decisions in R\&D: small technology based firms in the United States and Japan, in: IEEE Transations on Engineering Management, 44(2), S. 124-134. 
Lee, Ch.-Y. (2003a), A simple theory and evidence on the determinants of firm R\&D, in: Economics of Innovation and New Technology, 12(5), S. 385-395.

Lee, Ch.-Y. (2003b), Firm density and industry R\&D intensity: theory and evidence, in: Review of Industrial Organization, 22(2), S. 139-158.

Legler, H./Grenzmann, Ch./Marquandt R. (2006), Forschungs- und Entwicklungsaktivitäten der deutschen Wirtschaft im vergangenen Vierteljahrhundert, Studien zum deutschen Innovationssystem Nr. 2-2006.

Legler, H./Krawczyk, O. (2006), Forschungs- und Entwicklungsaktivitäten im internationalen Vergleich, Studien zum deutschen Innovationssystem Nr. 1-2006.

Leiponen, A. (2002), Why do firms not collaborate? The role of competencies and technological regimes, in: Kleinknecht, A.H./Mohnen, P.A. (Hrsg.), Innovation and firm performance: econometric explorations of survey data, Basingstoke [u.a.]: Palgrave, S. 253-277.

Lenz, S. (1998), Bestimmungsfaktoren des Innovations- und Kooperationsverhaltens von Unternehmen: Theorie und ökonometrische Untersuchung anhand von Daten für die schweizerische Industrie, Dissertation, ETH Zürich.

Leppälä, K. (1995), Inside a contract research laboratory: a study of concepts, methods and performance, Espoo: Technical Research Centre of Finland.

Levin, R.C./Cohen, W.M./Mowery, D.C. (1985), R\&D appropriability, opportunity, and market structure: new evidence on some Schumpeterian hypothesis, in: American Economic Review, 75(2), S. 20-24.

Levin, R.C./ Klevorick, A.K./ Nelson, R.R./ Winter, S.G. (1987), Appropriating the returns from industrial research and development, in: Brookings Papers on Economic Activity, 3, S. 783-820.

Levin, R.C./Reiss, P.C. (1984), Tests of a Schumpeterian model of R\&D and market structure, in: Griliches, Z. (Hrsg.), R\&D, patents, and productivity, Chicago [u.a.]: The Univ. of Chicago Press, S. 175-204.

Liao, T.F. (1994), Interpreting probability models: logit, probit and other generalized linear models, Thousand Oaks [u.a.]: Sage. 
Licht, G./Zoz, K. (2000), Patents and R\&D: an econometric investigation using applications for German, European and US Patents by German companies, in: Encaoua, D./Hall, B.H./Laisney, F./Mairesse, J. (Hrsg.), The economics and econometrics of innovation, Boston, Mass. [u.a.]: Kluwer Acad. Publ., S. 307-338.

Lieske, J. (2000), Forschung als Geschäft: Die Entwicklung von Auftragsforschung in den USA und Deutschland, Frankfurt a. Main [u.a]: Campus Verlag.

Long, J.S. (1997), Regression models for categorical and limited dependent variables, Thousand Oaks [u.a.]: Sage.

Long, J.S./Freese, J. (2001), Regression models for categorical dependent variables using Stata, College Station, Texas: Stata Press.

Madhok, A. (2002), Reassessing the fundamentals and beyond: Ronald Coase, the transaction cost and resource-based theories of the firm and the institutional structure of production, in: Strategic Management Journal, 23(6), S. 535-550.

Martiensen, J. (2000), Institutionenökonomik: die Analyse der Bedeutung von Regeln und Organisationen für die Effizienz ökonomischer Tauschbeziehungen, München: Vahlen.

Maskin, E./Tirole, J. (1999), Unforeseen contingencies and incomplete contracts, in: Review of Economic Studies, 66(226), S. 83-114.

Meschede, M. (2003), Produkt- und Prozessinnovationen in großen und kleinen Unternehmen, Frankfurt am Main [u.a.]: Lang.

Meuse, K.P. de/Lounsbury, J.W./Gordon, S.R. (1981), Assessing an $\mathrm{R} \& \mathrm{D}$ contract management process, in: Research Management, 24, S. 30-35.

Meza, D. de/Lockwood, B. (1998), Does asset ownership always motivate managers? Outside options and the property rights theory of the firm, in: Quarterly Journal of Economics, 113(2), S. 361-386.

Moore, J. (1992), The firm as a collection of assets, in: European Economic Review, 36(2), S. 493-507. 
Mowery, D.C. (1983), The relationship between intrafirm and contractual forms of industrial research in American manufacturing, 1900-1940, in: Explorations in Economic History, 20(4), S. 351-374.

Mowery, D./Rosenberg, N. (1979), The influence of market demand upon innovation: a critical review of some recent empirical studies, in: Research Policy, 8(2), S. 102-153.

Musgrave, R.A./Musgrave, P.B./Kullmer, L. (1975), Die öffentlichen Finanzen in Theorie und Praxis, Bd. 1, Tübingen: Mohr.

Nagarajan, A./Mitchell, W. (1998), Evolutionary diffusion: internal and external methods used to acquire encompassing, complementary, and incremental technological changes in the lithotripsy industry, in: Strategic Management Journal, 19(11), S. 1063-1078.

Narula, R. (2001), Choosing between internal and non-internal R\&D activities: some technological and economic factors, in: Technology Analysis \& Strategic Management, 13(3), S. 365-385.

Nelson, R.R. (1959), The simple economics of basic scientific research, in: Journal of Political Economy, 67(3), S. 297-306.

Nonaka, I. (1994), A dynamic theory of organizational knowledge creation, in: Organization Science, 5(1), S. 14-37.

Noorderhaven, N.G. (1995), The argumentational texture of transaction cost economics, in: Organization Studies, 16(4), S. 605-624.

Nooteboom, B./Vossen, R.W. (1995), Firm size and efficiency in R\&D spending, in: Witteloostuijn, A. (Hrsg.), Market evolution: competition \& cooperation, Dordrecht [u.a.]: Kluwer Acad. Publ., S. 69-86.

Nuhn, B. (1987), Eigen- und/oder Fremdforschung und -entwicklung als strategisches Entscheidungsproblem, Giessen: Ferber.

OECD (Hrsg.) (2002), Frascati manual 2002: the measurement of scientific and technological activities; proposed standard practice for surveys of research and experimental development, 6. ed, Paris.

OECD/Eurostat (Hrsg.) (1997), Oslo manual: proposed guidelines for collecting and interpreting technological innovation data, Paris. 
Ophem, H. van/Brouwer, E./Kleinknecht, A.H./Mohnen, P. (2000), The mutual relation between patents and $R \& D$, in: Kleinknecht, A.H./Mohnen, P.A. (Hrsg.), Innovation and firm performance: econometric explorations of survey data, Basingstoke, Hampshire [u.a.]: Palgrave S. $56-70$.

Pavitt, K. (1984), Sectoral patterns of technical change: towards a taxonomy and a theory, in: Research Policy, 13(6), S. 343-373.

Penzkofer, H. (2004), Innovationstest: Umfragen des ifo Instituts; Konzeption, Reprsentation, Qualittssicherung, in: Goldrian, G. (Hrsg.), Handbuch der umfragebasierten Konjunkturforschung, München: Ifo-Institut für Wirtschaftsforschung, S. 148-159.

Pfaffmann, E. (1999), Die Grenzen des Transaktionskostenansatzes: Einige kritische Anmerkungen zu Gestaltungsempfehlungen im Kontext von Make-or-Buy-Entscheidungen, in: Wirtschaftswissenschaftliches Studium, 28(11), S. 616-619.

Phlips, L. (1995), Competition policy: a game-theoretic perspective, Cambridge [u.a.]: Cambridge Univ. Press.

Picot, A./Dietl, H. (1990), Transaktionskostentheorie, in: Wirtschaftswissenschaftliches Studium, 19(4), S. 178-184.

Pisano, G.P. (1990), The R\&D boundaries of the firm: an empirical analysis, in: Administrative Science Quarterly, 35, S. 153-176.

Pisano, G.P. (1991), The governance of innovation: vertical integration and collaborative arrangements in the biotechnology industry, in: Research Policy, 20(3), S. 237-249.

Polanyi, M. (1958), Personal knowledge: toward a post-critical philosophy, Chicago: University of Chicago Press.

Poyago-Theotoky, J. (1999), A note on endogenous spillovers in a nontournament R\&D duopoly, in: Review of Industrial Organization, 15(3), S. 253-262.

Pregibon, D. (1981), Logistic regression diagnostics, in: The Annals of Statistics, 9(4), S. 705-724. 
Radnor, M. (1991), Technology acquisition strategies and processes: a reconsideration of the 'make versus buy' decision, in: International Journal of Technology Management, Special Publication on the Role of Technology in Corporate Policy, S. 113-135.

Raftery, A.E. (1996), Bayesian model selection in social research, in: Sociological Methodology, 25, S. 111-163.

Reiß, Th./Hüsing, B. (1992), Potenzialanalyse für Auftragsforschung in der Biotechnologie: Endbericht, Karlsruhe: Fraunhofer ISI.

Reitzig, M. (2004), The private values of 'thickets' and 'fences': towards an updated picture of the use of patents across industries, in: Economics of Innovation and New Technology, 13(5), S. 457-476.

Ringe, M.J. (1991), The contract research business in the UK, Science and Engineering Policy Studies Unit (SEPSU) Policy Study No. 6.

Rinne, H. (2000), Statistische Analyse multivariater Daten: Einführung, München [u.a.]: Oldenbourg.

Rosenkranz, S./Schmitz, P.W. (1999), Know-how disclosure and incomplete contracts, in: Economic Letters, 63(2), S. 181-185.

Rothaermel, F.T. (2001), Complementary assets, strategic alliances, and the incubent's advantage: an empirical study of industry and firm effects in the biopharmaceutical industry, in: Research Policy, 30(8), S. 12351251.

Röthlingshöfer, K.Ch. (1972), Die Vergabe von Forschungs- und Entwicklungsaufträgen in der Bundesrepublik Deutschland: empirische Untersuchung über Art, Umfang und Bedeutung der Vertragsforschung und -entwicklung, Berlin: Duncker \& Humblot.

Rothwell, R./Dodgson, M. (1991), External linkages and innovation in small and medium-sized enterprises, in: R\&D Management, 21(2), S. 125-137.

Rüdiger, M. (2000), Forschung und Entwicklung als Dienstleistung: Grundlagen und Erfolgsbedingungen der Vertragsforschung, Wiesbaden: Dt. Univ.-Verl. 
Rüdiger, M./Vanini, S. (1998), Das Tacit knowledge-Phänomen und seine Implikationen für das Innovationsmanagement, in: Die Betriebswirtschaft, 58(4), S. 467-480.

Santarelli, E./Sterlacchini, A. (1990), Innovation, formal vs. informal $\mathrm{R} \& \mathrm{D}$, and firm size: some evidence from Italian manufacturing firms, in: Small Business Economics, 2(3), S. 223-228.

Sattler, H. (2003), Appropriability of product innovations: an empirical analysis for Germany, in: International Journal of Technology Management, 26(5-6), S. 502-516.

Saussier, S. (2000), When incomplete contract theory meets transaction cost economics: a test, in: Menard, C. (Hrsg.), Institutions, contracts and organizations: perspectives from new institutional economics, Cheltenham [u.a.]: Elgar, S. 376-398.

Scherer, F.M. (1965), Firm size, market structure, opportunity and the output of patented inventions, in: American Economic Review, 55(5), S. 1097-1125.

Scherer, F.M. (1967), Market structure and the employment of scientists and engineers, in: American Economic Review, 57(3), S. 524-531.

Scherer, F.M. (1992), Schumpeter and plausible capitalism, in: Journal of Economic Literature, 30(3), S. 1416-1433.

Schmidt, I./Elßer, S. (1990), Innovationsoptimale Unternehmensgrößen und Marktstrukturen: die Neo-Schumpeter Hypothesen, in: Wirtschaftswissenschaftliches Studium, 19(11), S. 556-562.

Schmidt-Tiedemann, K.J. (1982), A new model of the innovation process, in: Research Management, 25, S. 18-21.

Schmitz, P.W. (2001), The hold-up problem and incomplete contracts: a survey of recent topics in contract theory, in: Bulletin of Economic Research, 53(1), S. 1-17.

Schmookler, J. (1966), Invention and economic growth, Cambridge, Mass.[u.a.]: Harvard Univ. Press.

Schneider, D. (1985), Die Unhaltbarkeit des Transaktionskostenansatzes für die „Markt oder Unternehmung"-Diskussion, in: Zeitschrift für Betriebswirtschaft, 55 (12), S.1237-1254. 
Schneider, D./Zieringer, C. (1991), Make-or-Buy-Strategien für F\&E: transaktionskostenorientierte Überlegungen, Wiesbaden: Gabler.

Schumpeter, J.A. (1911/1993), Theorie der wirtschaftlichen Entwicklung: Eine Untersuchung über Unternehmergewinn, Kapital, Kredit, Zins und den Konjunkturzyklus, 8. Aufl., Berlin: Duncker \& Humblot.

Schumpeter, J.A. (1942/1987), Kapitalismus, Sozialismus, Demokratie, 6. Aufl., Tübingen [u.a.]: Francke.

Schweizer, U. (1999), Vertragstheorie, Tübingen: Mohr Siebeck.

Scott, J.T. (1984), Firm versus industry variability in R\&D intensity, in: Griliches, Z. (Hrsg.), R\&D, patents, and productivity, Chicago [u.a.]: The Univ. of Chicago Press, S. 233-248.

Shapiro, C. (2001), Navigating the patent thicket: cross licenses, patent pools, and standard setting, in: Jaffe, A.B./Lerner, J./Stern, S. (Hrsg.), Innovation policy and the economy, Boston: MIT Press, S. 119-150.

Siddharthan, N.S./Agarwal, R.N. (1992), Determinants of R\&D decisions: a cross-section study of Indian private corporate firms, in: Economics of Innovation and New Technology, 2, S. 103-110.

Siemer, J.Ph. (2004), Vertikale Integration oder Kooperation?: optimale strategische Entscheidungen im Spannungsfeld von Spezifität und Knowhow, Wiesbaden: Deutscher Universitäts-Verlag.

Slater, G./Spencer, D.A. (2000), The uncertain foundations of transaction costs economics, in: Journal of Economic Issues, 34(1), S. 61-87.

Specht, G./Beckmann, Ch. (1996), F\&E-Management, Stuttgart: Schäffer-Poeschel.

Statistisches Bundesamt (Hrsg.) (2005), Fachserie 4, Reihe 4.3: Kostenstruktur der Unternehmen des Verarbeitenden Gewerbes sowie des Bergbaus und der Gewinnung von Steinen und Erden, Wiesbaden.

Stifterverband für die Deutsche Wissenschaft (Hrsg.) (2001), FuE-

Datenreport: Forschung und Entwicklung in der Wirtschaft 1999-2000; Bericht über die FuE-Erhebung 1999, Essen: Wissenschaftsstatistik $\mathrm{GmbH}$. 
Stifterverband für die Deutsche Wissenschaft (Hrsg.) (2004), FuEDatenreport: Forschung und Entwicklung in der Wirtschaft; Bericht über die FuE-Erhebungen 2001 und 2002, Essen: Wissenschaftsstatistik $\mathrm{GmbH}$.

Stiglitz, J.E. (1987), Technological change, sunk costs, and competition, in: Brookings Papers on Economic Activity, 3, S. 883-947.

Stiglitz, J.E. (1999), Volkswirtschaftslehre, 2. Aufl. (1.dt.-sprachige Aufl.), München [u.a.] : Oldenbourg.

Symeonidis, G. (1996), Innovation, firm size and market structure: Schumpeterian hypotheses and some new themes, Paris: OECD.

Takii, K. (2004), A barrier to the diffusion of tacit knowledge, in: Review of Development Economics, 8(1), S. 81-90.

Tapon, F. (1989), A transaction cost analysis of innovations in the organisation of pharmaceutical R\&D, in: Journal of Economic Behavior and Organization, 12(2), S. 197-213.

Tapon, F./Cadsby, Ch.B. (1996), The optimal organization of research: evidence from eight case studies of pharmaceutical firms, in: Journal of Economic Behavior and Organization, 31(3), S. 381-399.

Tapon, F./Thong, M. (1997), Outsourcing of research by pharmaceutical and biotechnology firms: 1988-1996, University of Guelph Depertment of Economics Dicussion Paper No. 1997-2.

Teece, D.J. (1988), Technological change and the nature of the firm, in: Dosi, G./Freeman, Ch./Nelson, R./Silverberg, G./Soete, L.(Hrsg.), Technical change and economic theory, London [u.a.]: Pinter, S. 256-281.

Terberger, E. (1994), Neo-institutionalistische Ansätze: Entstehung und Wandel - Anspruch und Wirklichkeit, Wiesbaden: Gabler.

Tietzel, M. (1981), Die Ökonomie der Property Rights: ein Überblick, in: Zeitschrift für Wirtschaftspolitik, 30(3), S. 207-243.

Tirole, J. (1999), Incomplete contracts: where do we stand?, in: Econometrica, 67(4), S. 74-782. 
Traill, I.E./Miege, R. (1989), Contract research organizations in the EEC: study and directory carried out for the Sprint Programme by Bossard Consultants, Paris, Luxembourg: Office for Official Publications of the European Communities.

UNCTAD (Hrsg.) (2000), World investment report 2000: cross-border mergers and acquisitions and development, New York [u.a.]: United Nations.

UNCTAD (Hrsg.) (2005), World investment report 2005: transnational corporations and the internationalization of R\&D, New York [u.a.]: United Nations.

Veugelers, R. (1997), Internal R\&D expenditures and external technology sourcing, in: Research Policy, 26(3), S. 303-315.

Veugelers, R. (1998), Collaboration in R\&D: an assessment of theoretical and empirical findings, in: De Economist, 146(3), S. 419-443.

Veugelers, R./Cassiman, B. (1999), Make and buy in innovation strategies: evidence from Belgian manufacturing firms, in: Research Policy, 28(1), S. 63-80.

Völker, R./Kasper, E. (2004), Interne Märkte in Forschung und Entwicklung, Heidelberg [u.a.]: Physica-Verl.

Vonortas, N.S. (1994), Inter-firm cooperation with imperfectly appropriable research, in: International Journal of Industrial Organization, 12(3), S. 413-435.

Vossen, R.W. (1999), Market power, industrial concentration and innovative activity, in: Review of Industrial Organization, 15(4), S. 367-378.

Vossen, R.W./Nooteboom, B. (1996), Firm size and participation in R\&D, in: Kleinknecht, A.H. (Hrsg.), Determinants of innovation: the message from new indicators, Basingstoke [u.a.]: Macmillan, S. 155-168.

Williamson, O.E. (1981), The modern corporation: origings, evolution, attributes, in: Journal of Economic Literature, 19(4), S. 1537-1568.

Williamson, O.E. (1988), Technology and transaction cost economics: a reply, in: Journal of Economic Behavior and Organization, 10(3), S. 355363. 
Williamson, O.E. (1989), Transaction cost economics, in: Schmalensee, R./Willig R.D. (Hrsg.), Handbook of industrial innovation, Vol. I, Amsterdam [u.a.]: North-Holland, S. 135-182.

Williamson, O.E. (1990), Die ökonomischen Institutionen des Kapitalismus; Unternehmen, Märkte, Kooperationen, Tübingen: Mohr.

Williamson, O.E. (1991a), Comparative economic organization: vergleichende ökonomische Organisationstheorie; die Analyse diskreter Strukturalternativen, in: Ordelheide, D./Bernd, R./Büsselmann, E. (Hrsg.), Betriebswirtschaftslehre und ökonomische Theorie, Stuttgart: Poeschel, S. 13-49.

Williamson, O.E. (1991b), Comparative economic organization: the analysis of discrete structural alternatives, in: Administrative Science Quaterly, 36, S. 269-296.

Williamson, O.E. (1993), The economic analysis of institutions and organisations: in general and with respect to country studies, OECD Economics Department Working Paper No. 133.

Williamson, O.E. (1996), The mechanisms of governance, New York [u.a.]: Oxford University Press.

Williamson, O.E. (2000), The new institutional economics: taking stock, looking ahead, in: Journal of Economic Literature, 38(3), S. 595-613.

Windsperger, J. (1997), Zur Methode des Transaktionskostenansatzes: Replik auf Dieter Schneider's Untersuchung über „Die Unhaltbarkeit des Transaktionskostenansatzes für die "Markt-oder-Unternehmung“Diskussion", in: Zeitschrift für Betriebswirtschaft, 57(1), S. 59-76.

Windsperger, J. (1998), Ungelöste Probleme der Transaktionskostentheorie, in: Journal für Betriebswirtschaft, 48(5-6), S. 266-276.

Zumbroich, Th.J. / Gadicke, A.S. / Steiner, M. (1995), Strategische Neuorientierung der pharmazeutischen Forschung und Lizenzierung, in: Herzog, R. (Hrsg.), F\&E-Management in der Pharma-Industrie, Aulendorf: ECV - Editio-Cantor-Verl., S. 328-338. 
Katarzyna Haverkamp - 978-3-631-75689-8

Downloaded from PubFactory at 01/11/2019 02:56:45AM

via free access 


\section{CeGE-Schriften}

Das CeGE - Centrum für Globalisierung und Europäisierung der Wirtschaft - wurde 1999 von der Wirtschaftswissenschaftlichen Fakultät der Georg-August-Universität Göttingen gegründet. Das Centrum dient als Forum zur internationalen und interdisziplinären Zusammenarbeit bei der Analyse ökonomischer Fragestellungen von europäischer oder globaler Bedeutung. In den CeGE-Schriften werden Forschungsergebnisse aus Dissertationen, Habilitationen und anderen Forschungsprojekten des Zentrums veröffentlicht.

Band 1 Axel Gerloff: Wechselkurspolitik in Mittel- und Osteuropa. Eine theoretische Analyse unter besonderer Berücksichtigung der Enfahrungen der zehn Kandidaten für eine EU-Osterweiterung. 2001.

Band 2 Ingo Konrad: Zur Integration ausgewăhlter mittel- und osteuropäischer Lảnder in die wăhrungspolitische Ordnung Europas. 2002.

Band 3 Axel Hennighausen: Wegekostenfinanzierung und Lenkung im deregulierten europäischen Verkehrsmarkt. 2002.

Band 4 Wolfgang Münch: Effects of EU Enlargement to the Central European Countries on Agricultural Markets. 2002.

Band 5 Holger Niermann: Welchen Finanzausgleich braucht Europa? Ein konkreter Vorschlag zur Neugestaltung der europăischen Finanzbeziehungen mit Simulationsmodell. 2002.

Band 6 Jens Sũdekum: Agglomeration and Regional Unemployment Disparities. A Theoretical Analysis with Reference to the European Union. 2003.

Band 7 Christoph Schinke: Der Geldmarkt im Euro-Wăhrungsraum. Geldmarktgeschäfte, Zinsbildung und die Taylor Rule. 2004.

Band 8 Kai Stukenbrock: The Stability of Currency Boards. 2004.

Band 9 Harald Grethe: Effects of Including Agricultural Products in the Customs Union between Turkey and the EU. A Partial Equilibrium Analysis for Turkey. 2004.

Band 10 Götz Zeddies: Perspektiven der Gemeinschaftspolitiken in der enweiterten EU. 2005.

Band 11 Ingmar Kumpmann: Systemwettbewerb und Umverteilung. Gefährdet die Globalisierung den Sozialstaat? 2005.

Band 12 Katarzyna Haverkamp: Bestimmungsfaktoren der Vertragsforschung in Deutschland. Eine theoretische und ókonometrische Analyse. 2007

www.peterlang.de 


\section{Innovationen kleiner Unternehmen in regionalen Netzwerken}

\section{Die Förderung von Forschung und Entwicklung durch aktive Vermittlung im Wissens- und Technologietransfer}

Frankfurt am Main, Berlin, Bern, Bruxelles, New York, Oxford, Wien, 2004. 296 S., zahlr. Abb. und Tab.

Strukturwandel und Strukturpolitik. Herausgegeben von Wolfram Elsner. Bd. 7 ISBN 978-3-631-52318-6 · br. € 51.50*

Kleine Unternehmen sind in ihrem Innovationsverhalten verschiedenen Restriktionen unterworfen. Die Einbindung in ein regionales Innovationssystem gilt als wichtiges Instrument zur Überwindung mangelnder Ressourcen. Insbesondere die aktive Vermittlung im Wissens- und Technologietransfer zu regionalen Wissensgebern aus Hochschulen und öffentlichen Einrichtungen kann Innovationen unterstützen. Eine Fallstudie von 268 Vermittlungen in einem vorwiegend peripher gelegenen Landkreis beleuchtet Einflussfaktoren und Randbedingungen für das Entstehen von Kooperationen und weist auf Basis der empirisch gewonnenen Daten nach, wie vormalige OutsiderUnternehmen an das Subsystem Wissenschaft herangeführt werden können.

Aus dem Inhalt: Kleine Unternehmen - Regionale Innovationssysteme · Wissens- und Technologietransfer - Innovationsförderung · Aktive, regionale Vermittlung und Netzwerkmoderation - Kooperationen Wirtschaft - Wissenschaft - Verbesserung der Adaptionsfähigkeit kleiner Unternehmen · Heranführung an das Subsystem Wissenschaft · Empirische Untersuchung von Einflussfaktoren und Randbedingungen - Innovationsfähigkeit von Regionen - Der Beispiellandkreis Diepholz als Normalregion

Frankfurt am Main - Berlin - Bern - Bruxelles - New York - Oxford - Wien

Auslieferung: Verlag Peter Lang AG

Moosstr. 1, $\mathrm{CH}-2542$ Pieterlen

Telefax 0041 (0) $32 / 3761727$

*inklusive der in Deutschland gültigen Mehrwertsteuer

Preisänderungen vorbehalten

Homepage http://www.peterlang.de 